Categorical quantum models and logics 
The work in this thesis has been carried out while the author was employed at the Radboud University Nijmegen, financially supported by the Netherlands Organisation for Scientific Research (NWO) within the Pionier projects "Program security and correctness" during August 2005-August 2007, and "Quantization, noncommutative geometry and symmetry" during August 2007-August 2009.

Typeset using $\mathrm{HT}_{\mathrm{E}} \mathrm{X}$ and $\mathrm{X}$-pic

ISBN 9789085550242

NUR 910

(C) C. Heunen / Pallas Publications - Amsterdam University Press, 2009

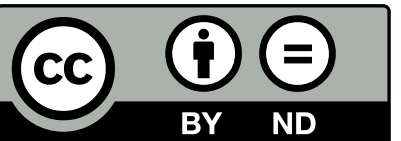

This work is licensed under a Creative Commons Attribution-No Derivative Works 3.0 Netherlands License. To view a copy of this license, visit: http://creativecommons.org/licenses/by-nd/3.0/nl/. 


\section{Categorical quantum models and logics}

een wetenschappelijke proeve op het gebied van de Natuurwetenschappen, Wiskunde en Informatica

\section{PROEFSCHRIFT}

ter verkrijging van de graad van doctor

aan de Radboud Universiteit Nijmegen

op gezag van de rector magnificus prof. mr. S.C.J.J. Kortmann, volgens besluit van het College van Decanen in het openbaar te verdedigen op 7 januari 2010 om 13.30 uur precies

door

Christiaan Johan Marie Heunen

geboren op 21 maart 1982

te Nijmegen 


\section{Promotores:}

prof. dr. B.P.F. Jacobs

prof. dr. N.P.L. Landsman

Doctoral thesis committee:

prof. dr. S. Abramsky University of Oxford

prof. dr. M. Gehrke Radboud University Nijmegen

prof. dr. P.T. Johnstone University of Cambridge

prof. dr. I. Moerdijk Utrecht University

dr. M. Müger Radboud University Nijmegen 


\section{Preface}

I cannot allow this thesis to be published without thanking those without whom it could not have been.

First and foremost, I am deeply grateful to my promotores. Bart Jacobs, ever cheerful, enthusiastic and ready to explain, taught me the importance of "thinking with one's fingers". From Klaas Landsman I learned the value of "social science", i.e. how rigorous research can be jump-started by opinions of experts on vague ideas. I am very glad to have had the opportunity to work with such amicable supervisors, and can only hope that their invaluable guidance is reflected in this thesis. I am also honoured by the effort that the members of the doctoral committee put into reading my work. Especially Peter Johnstone, whose remarks were spot on and reveal a very careful reading, saved me from eternal shame, for which I thank him heartily.

In addition to my supervisors, I am indebted to my other co-authors Bas Spitters, Ichiro Hasuo, Ana Sokolova and Martijn Caspers for sharing their insight. Especially Bas came up with incomprehensibly many ideas to work out. Furthermore, my colleagues in the research community always made me feel very welcome, not only during all those conferences and workshops. In particular, I enjoyed the encouragement, constructive criticisms and advice by John Harding, Isar Stubbe, Jamie Vicary, and Steve Vickers. In the same spirit, I thank Bob Coecke, Marcelo Fiore and Ichiro Hasuo for inviting me on research visits to Oxford, Cambridge and Kyoto, respectively, during which I learned a great deal.

Closer to home, I am grateful to all my colleagues at the mathematics department, who were always ready to answer my probably blatantly obvious questions. The digital security group provided a much appreciated lively atmosphere, despite my research topic not quite fitting in seamlessly. This homely feeling is largely due to Miguel Andres, Łukasz Chmielewski, Flavio Garcia, Ichiro Hasuo, Ron van Kesteren, Gerhard de Koning Gans, Ken Madlener, Peter van Rossum, Ana Sokolova, and Alejandro Tamalet, with all of whom I shared an office over the years. To be fair, it was not only tea and table football: there were certainly also research discussions and reading groups, especially with Ana, Ichiro and 
Peter, which I very much appreciated. Lastly, Wojciech Mostowski was always ready to share his intimate knowledge of $\mathrm{T}_{\mathrm{E}} \mathrm{X}$ when mine wasn't deep enough.

The support I enjoyed from outside academia was perhaps just as important, and I would like to thank all my friends for their companionship over the last years, including the (Roman) dinners, movie nights, sailing weekends, snowboarding trips, and general amusement. Łukasz and Ron in addition accepted the task of being my paranymphs. There are too many more friends to list here, but in particular, the members of karate clubs NSKV Dojo and Shu Ken Ma Shi should be mentioned, as the countless training hours with them provided a lot of fun and relief. Let me conclude with some words of gratitude to my family (in-law) for their care in my development; especially to my brothers, for keeping my feet on the ground, and to my parents, for always being there to help, even with the most practical of things. Finally, and most of all, I thank Lotte, for so much more than can be mentioned here.

Utrecht, August 2009 


\section{Contents}

\begin{tabular}{lll}
\hline 1 & Introduction & 1
\end{tabular}

$\begin{array}{lll}2 & \text { Tensors and biproducts } & 11\end{array}$

2.1 Examples . . . . . . . . . . . . . . . . . . . . . 11

2.2 Tensor products and monoids $\ldots \ldots \ldots \ldots \ldots$

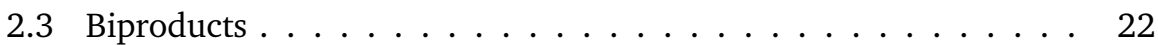

2.4 Scalars . . . . . . . . . . . . . . . . . . . . . . . . . 29

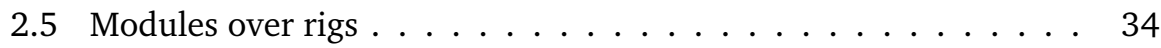

2.6 Compact objects $\ldots \ldots \ldots \ldots \ldots \ldots$. . . . . . . . . . . . . 41

\begin{tabular}{|lll}
\hline & Dagger categories & 47
\end{tabular}

3.1 Examples . . . . . . . . . . . . . . . . . . 47

3.2 Dagger structures $\ldots \ldots \ldots \ldots \ldots \ldots$

3.3 Quantum key distribution $\ldots \ldots \ldots \ldots$. . . . . . . 66

3.4 Factorisation $\ldots \ldots \ldots \ldots \ldots \ldots \ldots \ldots$

3.5 Hilbert modules . . . . . . . . . . . . . . . . . . . . . . 80

3.6 Scalars revisited $\ldots \ldots \ldots \ldots . \ldots \ldots$

3.7 Hilbert categories . . . . . . . . . . . . . . . . . . . . 90

$\begin{array}{lll}4 & \text { Dagger kernel logic } & 99\end{array}$

4.1 Subobjects . . . . . . . . . . . . . . . . . . . . . . . . . . 999

4.2 Orthogonality . . . . . . . . . . . . . . . . . 106

4.3 Orthomodularity $\ldots \ldots \ldots \ldots \ldots \ldots \ldots$

4.4 Quantifiers . . . . . . . . . . . . . . . . . . . . 117

4.5 Booleanness . . . . . . . . . . . . . . . . . . . . . 127

4.6 Subobject classifiers $\ldots \ldots \ldots \ldots \ldots$

5 Bohrification 141

5.1 Locales and toposes . . . . . . . . . . . . . . . . . . . . . . . . . . . . . . 141

$5.2 \mathrm{C}^{*}$-algebras $\ldots \ldots \ldots \ldots \ldots \ldots \ldots \ldots$ 
5.3 Bohrification $\ldots \ldots \ldots \ldots \ldots \ldots \ldots \ldots \ldots \ldots \ldots$

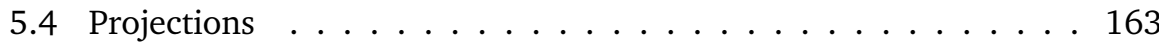

5.5 States and observables . . . . . . . . . . . . . . . . 172

\begin{tabular}{ll}
\hline Bibliography & 181
\end{tabular}

Index of categories 195

Index of notation 197

\begin{tabular}{ll}
\hline Index of subjects & 199
\end{tabular}

\begin{tabular}{ll}
\hline Samenvatting & 203
\end{tabular} 


\section{Chapter 1}

\section{Introduction}

Quantum theory is the best description of nature at very small scales to date. Its principal new features compared to classical physics are superposition of states, noncommutativity of observables, and entanglement. Although strange and counterintuitive at first, such features can be exploited once recognised. Entanglement, for example, was discovered and regarded as a paradox by Einstein, Podolsky and Rosen in 1935 [79], but nowadays it is mainly seen as a resource to be used. For example, entanglement enables key distribution protocols, providing each of the participating parties with a string of bits that is guaranteed to be known to them only. Even more so, quantum computers employ entanglement to solve certain problems essentially faster than a classical computer can [173].

To achieve their full potential, such new applications have to come with mathematical proofs. Nobody will use a quantum computer for serious tasks if the programmer cannot vouch for the correctness of the program, and the very attractiveness of quantum key distribution for secret communication lies in the guarantee that there can be no eavesdroppers. Because human intuition is unreliable in the quantum world, a mathematically rigorous way to reason about quantum situations is called for. In other words, we need a logic for quantum physics, and that is what this thesis investigates.

\section{Counterintuitive features of quantum physics}

To illustrate the counterintuitive features of quantum physics, let us first explain the general form of any physical theory. An isolated object is described by the set of states in which it can be, and its empirical properties are modeled by a set of observables, so that a state and an observable can be combined into a real value, modeling the outcome of the act of observation, i.e. measurement. Often, we are not sure about the exact state of an object. Therefore we allow convex combina- 
tions of states. Observation (the pairing of a state with an observable) then only results in a given value with a certain probability. The states about which we have perfect knowledge, i.e. which cannot be written as convex combinations of other states, are called pure states. Finally, there is some way to combine the state spaces of component objects into the state space for a compound object.

The above scheme finds a natural home in classical physics, where the pure states of an object form a set $X$. It might come with a topology or some geometric structure, but in principle $X$ is just a set. Observables are functions $f: X \rightarrow \mathbb{R}$, such as speed. Observation of an object in a pure state $x \in X$ results in a sharp observed value $f(x)$. Elementary propositions, like "the object's speed is between 10 and $20 \mathrm{~m} / \mathrm{s}$ ", are dictated to be true in a pure state $x$, if and only if $f(x) \in(10,20)$, i.e. if and only if $x \in f^{-1}(10,20)$. The state of a compound object completely determines the states of its component objects: if $X$ and $Y$ are the state spaces of the component objects, then $X \times Y$ is the state space of the compound object.

The traditional formulation of quantum physics, which is due to John von Neumann [213], also fits the above scheme. The state space of a quantum system has the structure of a Hilbert space $X$. That is, $X$ comes with an inner product $\left\langle x \mid x^{\prime}\right\rangle$ that signifies the probability amplitude of a transition from state $x$ to state $x^{\prime}$. Pure states are unit vectors. Observables are self-adjoint operators $f: X \rightarrow X$. By the spectral theorem, which generalises diagonalisation of matrices, every such operator $f$ corresponds uniquely to a family of (so-called projection) operators $e_{\Delta}: X \rightarrow X$ for every interval $\Delta \subseteq \mathbb{R}$. In contrast to classical physics, even observation of an object in a pure state only gives probabilistic results. Elementary propositions, like "the object's speed is between 10 and 20 $\mathrm{m} / \mathrm{s}$ " hold with probability $\left\langle x \mid e_{(10,20)}(x)\right\rangle$ in state $x$. Objects are combined by tensor products: if $X$ and $Y$ are the state spaces of the component objects, then $X \otimes Y$ is the state space of the compound object.

The fact that a Hilbert space $X$ comes with an addition is the source of the principle of superposition. As in classical physics, pure states $x$ and $x^{\prime}$ can be mixed by convex combination into a state that is no longer pure in general. But linear combinations of $x$ and $x^{\prime}$ such as $x+x^{\prime}$ do yield pure states, in which the probability of the object's behaviour is not simply the sum of the probabilities of its behaviours in states $x$ and $x^{\prime}$. A famous example is Schrödinger's cat. Its state upon inspection can either be "alive" or "dead", making the fact that the superposition state ("alive" + "dead") is pure, i.e. represents perfect knowledge, counterintuitive.

A related circumstance is noncommutativity. In both classical and quantum physics, the observables have a particular algebraic structure modeling the simultaneous measurement of two observables. In the classical case, two observ- 
ables $f, g: X \rightrightarrows \mathbb{R}$ can be multiplied pointwise to obtain $f \cdot g: X \rightarrow \mathbb{R}$. This is evidently commutative: $f \cdot g=g \cdot f$. Alternatively, regarding $f$ and $g$ as matrices whose nonzero entries lie on the diagonal, multiplication becomes composition. In the quantum case, too, two observables $f, g: X \rightrightarrows X$ can be composed to get $g \circ f: X \rightarrow X$. But this operation is no longer commutative, giving rise to the counterintuitive fact that observables cannot always be measured simultaneously without mutual disturbance.

Finally, entanglement is caused by compound quantum systems being described by tensor products instead of Cartesian products. Hence component objects are linked, in the sense that the state of the one instantaneously determines the state of the other upon measurement, even when separated by a large distance. For a non-example, consider an insistent couple trying to get a job at the same institute. Each of them independently applies for a position every day, and each receives a response every day. Suppose that each finds the probability of acceptance to be one in twenty, but that if one applicant is accepted, so is the other. This could have been caused by a humane personnel officer sending matching letters at an erratic institute whose policy is to randomly hire one in twenty. Entanglement in quantum physics is counterintuitive because it can arise without a common cause.

\section{Categorical models}

The general forms of classical and quantum physics explained above equate isolated objects with their state spaces. Moreover, both settings consider multiple interacting objects by prescribing how to form (state spaces of) compound objects. In fact, both frameworks incorporate some relationships between the state spaces, as they define observables to be special kinds of functions. Therefore the use of category theory suggests itself. Indeed, we will take the above one step further, and consider all relationships between (state spaces of) objects. For example, one object's speed might directly influence another's, so that there is a function from the state space of the one to the state space of the other. So classical physics takes place in the category of sets and functions, whereas the category of Hilbert spaces and continuous linear transformations embodies quantum physics.

The first part of this thesis studies properties of categories that account for the most important qualitative aspects of quantum physics. By doing so in an axiomatic fashion, one gains clear understanding of what features are caused by what assumptions.

For example, we consider categories with tensor products, modeling the ability to form compound systems, including the single-state system $I$. The internal structure of an object can then be recovered, as states correspond to morphisms 
$x: I \rightarrow X$. As a special case, so-called biproducts in a category bring about the superposition principle, since they entail that parallel morphisms $f, g: X \rightrightarrows Y$, and in particular states $x, y: I \rightrightarrows X$, can be added to obtain another morphism $f+g: X \rightarrow Y$.

Entanglement requires a certain link between the component objects in addition to the ability to compose systems with tensor products $\otimes$. This can be expressed axiomatically by requiring $X$ to be a so-called compact object, so that it has a dual object $X^{*}$, together with which it forms the entangled compound system $X^{*} \otimes X$.

We also explore dagger categories, in which morphisms $f: X \rightarrow Y$ can be reversed to obtain $f^{\dagger}: Y \rightarrow X$. This models a phenomenon that already occurs in reversible computing - by the law of conservation of energy, any quantum computation should be reversible. (Classical computers dissipate heat and can therefore ignore it, whereas quantum computers have to address decoherence, the quantum analogue of this issue, to function properly at all.) More generally, a dagger on a category could be said to implement conservation of information.

We will prove that if a categorical model has superposition, entanglement, and a dagger, as described above, it necessarily embeds into the category of Hilbert spaces, under some additional technical assumptions.

\section{Logic in classical physics}

Having discussed both traditional models and our categorical models, we now move to logic, which is the topic of the second part of this thesis. Let us first review the classical case, in which we are led to consider elementary propositions as subsets $K$ of $X$, such as $f^{-1}(10,20)=\{x \in X \mid f(x) \in(10,20)\}$. Observables $f$ might be taken continuous or measurable, in which case $K$ is an open, or measurable, subset. But in general, $K$ is simply a subset of $X$, and therefore the logic of classical physics is encoded by the collection of $\mathcal{P}(X)$ of subsets of $X$. Hence $K$ is true in state $x$ if and only if $x \in K$. Conjunction $\wedge$ of elementary propositions then becomes intersection of sets, disjunction $\vee$ becomes union, and negation $\neg$ becomes complementation. The elementary proposition that never holds is the empty set, and the proposition that always holds is the set $X$ itself. Moreover, propositions can be ordered by inclusion, so that $K \leq L$ means that $L$ is true when $K$ is.

This procedure is unobjectionable, in that our logical intuition coincides with the structure of $\mathcal{P}(X)$ : 
- $K \vee L$ is true if and only if $K$ is true or $L$ is true;

- $K \wedge L$ is true if and only if $K$ is true and $L$ is true;

- $\neg K$ is true if and only if $K$ is not true;

- there is an implication $\Rightarrow: \mathcal{P}(X) \times \mathcal{P}(X) \rightarrow \mathcal{P}(X)$ satisfying

$$
K \wedge L \leq M \text { if and only if } K \leq(L \Rightarrow M),
$$

which intuitively equates deriving the conclusion $M$ from hypotheses $K$ and $L$, and deriving the conclusion that $L$ implies $M$ from the hypothesis $K$;

- conjunction distributes over conjunction:

$$
K \wedge(L \vee M)=(K \wedge L) \vee(K \wedge M)
$$

A proposition about the compound system consisting of two component objects becomes a subset $K$ of $X \times Y$. Hence we can consider predicates, for example, expressing that $K$ holds regardless of the state $x \in X$ of the first component object. Following, as before, the unobjectionable strategy of making the semantics of propositions coincide with our logical intuition, this predicate $\forall_{x \in X} . K$ becomes the subset $\{y \in Y \mid(x, y) \in K$ for all $x \in X\}$. Similarly, the predicate $\exists_{x \in X} . K$ becomes the subset $\{y \in Y \mid$ there is an $x \in X$ such that $(x, y) \in K\}$. In the category of sets and functions, categorical logic elegantly characterises these existential and universal quantifiers as left and right adjoints to pullback, respectively.

\section{Traditional quantum logic}

If we apply the blueprint of the logic of classical physics to quantum physics, then we are led to consider subsets of $X$ of the form $K=\left\{e_{(10,20)}(x) \mid x \in X\right\}$. Since these subsets are always closed subspaces, taking those to be the elementary propositions stands to reason. The structure of the state space $X$ as a Hilbert space again enables us to build further propositions from elementary ones by the operations on closed subspaces: one can form orthocomplements $K^{\perp}=$ $\left\{x \in X \mid\left\langle x \mid x^{\prime}\right\rangle=0\right.$ for all $\left.x^{\prime} \in K\right\}$ to be used as negations $\neg$, intersections $\wedge$, and closures of linear spans $\vee$. Directly interpreting this as a logic, however, is fraught with difficulties, mostly owing to the fact that the collection of closed subspaces $K$ of $X$ only form a so-called orthomodular (as opposed to Boolean) lattice: 
- there are superposition states in which $K \vee L$ is true while neither $K$ or $L$ is;

- there are propositions $K$ and $L$ for which the conjunction $K \wedge L$ makes no physical sense because the associated observables do not commute;

- $\neg K$ is true if and only if $K$ is false, i.e. if the probability that $K$ holds in state $x$ is zero, rather than if and only if $K$ is not true, i.e. the probability is less than one;

- there exists no map $\Rightarrow$ satisfying (1.1);

- $\vee$ and $\wedge$ do not distribute over each other-in an analogy due to Chris Isham, it is possible to get neither eggs and bacon nor eggs and ham for breakfast, when given a choice between eggs and either bacon or ham.

Moreover, the semantic interpretation of possible quantifiers in this setting is questionable. After all, due to entanglement, restricting a pure state of a compound system $X \otimes Y$ to the first component yields a state in $X$ that is no longer pure in general. This renders unclear how to assert predicates such as $\exists_{x \in X} . K$ for $K \subseteq X \otimes Y$. Despite these objections, the above enterprise, which is due to Garrett Birkhoff and John von Neumann, is traditionally called "quantum logic" [30].

We can model closed subspaces categorically as kernels. We will show that additionally requiring a dagger already suffices to recover this traditional quantum logic in our categorical models. Moreover, following the prescription of categorical logic to regard quantifiers as adjoints, we are able to establish an existential quantifier in such categories. We will also deduce that a universal quantifier cannot exist. However, the existential quantifier, although it exists, does not behave entirely as expected. In a sense, it has a rather "dynamic" or "temporal" character that is due to noncommutativity.

\section{Bohrification}

We circumvent the problem of noncommutativity by using categorical logic in a different way than directly applying it to our categorical models. A category that resembles the one of sets and functions sufficiently much to enable the interpretation of higher order intuitionistic logic, is called a topos. Toposes have the remarkable aspect that they not only embody logic, but are also generalisations of the concept of (topological) space.

Let us consider a special kind of the categorical models discussed so far, namely a $\mathrm{C}^{*}$-algebra $A$, which is possibly noncommutative. We will construct a specific topos $\mathcal{T}(A)$, and a canonical object $\underline{A}$ in it. The topos $\mathcal{T}(A)$ is based 
on an amalgamation of all the commutative $\mathrm{C}^{*}$-subalgebras $C$ of $A$. These can be seen as "contexts" or "classical snapshots of reality". This philosophy, due to Niels Bohr, came to be called his "doctrine of classical concepts" [193], whose best-known formulation is:

"However far the phenomena transcend the scope of classical physical explanation, the account of all evidence must be expressed in classical terms. (...) The argument is simply that by the word experiment we refer to a situation where we can tell others what we have done and what we have learned and that, therefore, the account of the experimental arrangements and of the results of the observations must be expressed in unambiguous language with suitable application of the terminology of classical physics." [32]

Moreover, at least according to our mathematical interpretation of complementarity [110, 150], the contexts $C$ together contain all physically relevant information contained in the quantum system $A$. Being an implementation of Bohr's philosophy, we call $\underline{A}$, or rather the process of obtaining it, Bohrification. Its importance lies in the fact that $\underline{A}$ is a commutative $\mathrm{C}^{*}$-algebra when seen from within the "universe of discourse" that is $\mathcal{T}(A)$. As such, it can be studied as if it consisted of observables of a classical physical system, though not living in the category of sets but in the unusual environment of the topos $\mathcal{T}(A)$. In particular, it has a state space $\underline{X}$. Stepping out of the topos $\mathcal{T}(A)$ again, $\underline{X}$ has an external description $X$, that does live in the usual category of sets, which we call the Bohrified state space of $A$. It comes with operations $\neg, \vee, \wedge$ that are defined "locally", i.e. through commutative parts, and therefore have no interpretational difficulties:

- $K \vee L$ is true if and only if $K$ is true or $L$ is true;

- the conjunction $K \wedge L$ is always defined physically, as it only involves "local" conjunctions, i.e. conjunctions of commuting observables;

- $\neg K$ is true if and only if $K$ is false;

- there is an implication $\Rightarrow: X \times X \rightarrow X$ satisfying (1.1).

- disjunction and conjunction distribute over each other.

Nevertheless, the logic that $X$ carries is not classical, but intuitionistic in nature. Moreover, $X$ carries a (generalised) topology, and therefore also shares the spatial aspects with state spaces in classical physics. 


\section{Outline and results}

To outline this thesis, let us list the central results of each chapter.

Chapter 2 shows that every category with tensor products as well as biproducts is enriched in modules over a so-called rig, and that this enrichment is functorial. Subsequently, this is used to show that such categories embed into a category of modules.

Chapter 3 in addition assumes a dagger, as well as further assumptions about equalisers and monomorphisms, and proves that such categories embed into the category of Hilbert spaces. In particular, the scalars in such a category always form an involutive field. This link to the traditional formalism is a satisfactory justification for considering the categorical models we study. As an intermezzo, this chapter also proves the correctness of a certain quantum key distribution protocol categorically.

Chapter 4 proves that kernel subobjects of a fixed object in a dagger kernel category form orthomodular lattices. As in Chapter 3, this parallels the situation in the traditional formalism of quantum logic. Subsequently, an existential quantifier is established; this has not been achieved in the traditional formalism.

Chapter 5 introduces the technique of Bohrification. The definition of Bohrification itself is closely connected to the chapter's main result, namely that any $\mathrm{C}^{*}$-algebra becomes commutative in its associated topos, and therefore has a spectrum in that topos. A large part of the chapter is devoted to determining that spectrum explicitly.

\section{Prerequisites}

As prerequisites we assume a working knowledge of basic category theory, including adjunctions, monoidal categories and enriched categories. Standard references are [33, 34, 141, 163]. A full appreciation of Chapter 4requires some familiarity with categorical logic [125, 146, 151, 154, 168, 208], but grosso modo the chapter can be understood without this knowledge. Likewise, Chapter 5 uses topos theory. We have strived to make this chapter understandable for readers without knowledge of the vast literature on this subject [25, 35, 95, 131, 164], which we cannot hope to even summarise in a single chapter.

About quantum theory we assume very little background knowledge. Some basic Hilbert space theory [181, 214] will probably aid the reader's intuition, 
but is otherwise unnecessary. Similarly, there is quite a body of work concerning operator algebras [56, 71, 135, 153, 206], which play a prominent role in Chapter 5, but in this respect the chapter should be self-contained.

To end this introduction, let us mention that we will not worry about size issues, that sometimes play a role in category theory. In particular, when enrichment is at play, we consider all categories to be locally small. This will not be a major problem, since most categories in this thesis are concrete. 



\section{Chapter 2}

\section{Tensors and biproducts}

This chapter studies monoidal structures in a category. The motivating category of modules over a ring has at least two such structures: tensor products and biproducts. Moreover, the former distributes over the latter. We will see that such structure in any category makes the homsets into modules over a rig, and that this enrichment proceeds in a functorial way. This eventually results in preparatory embedding theorems that pave the way for our big embedding theorem in Chapter 3 .

A lot of the developments in this chapter resemble the theory of Abelian categories [88, 170], or, more precisely, exact categories [19]. Most of the novel material in this chapter is based on [113].

\subsection{Examples}

We start this chapter by introducing several example categories that will be used throughout.

2.1.1 Example We denote the category of rings and ring homomorphisms by Rng, and the full subcategory of commutative rings by $\mathbf{c R n g}$. For a chosen $R \in \mathbf{R n g}$, a left- $R$-module is a set $X$ equipped with a commutative addition $(+, 0)$ and a scalar multiplication $: R \times X \rightarrow X$ satisfying the familiar equations. The equation $(r \cdot s) \cdot x=r \cdot(s \cdot x)$ for $r, s \in R$ and $x \in X$ explains the prefix 'left'. Analogously, a right-R-module has a scalar multiplication $: X \times R \rightarrow X$ satisfying $x \cdot(r \cdot s)=(x \cdot r) \cdot s$. A left- $R$-right-S-module is simultaneously a left- $R$ module and an right- $S$-module with the same addition and $(r \cdot x) \cdot s=r \cdot(x \cdot s)$. If $R$ is commutative, any left- $R$ - or right- $R$-module is automatically a left- $R$-right$R$-module, and we speak simply of an $R$-module. 
A morphism of left- $R$-modules is a function $f$ that is linear, i.e. that satisfies $f(x+y)=f(x)+f(y)$ and $f(r \cdot x)=r \cdot f(x)$. A morphism of right-modules similarly preserves the scalar multiplication on the right. Thus we get categories ${ }_{R} \operatorname{Mod}$ of left- $R$-modules, $\operatorname{Mod}_{R}$ of right- $R$-modules, and ${ }_{R} \operatorname{Mod}_{S}$ of left- $R$ right- $S$-modules. For $R \in \mathbf{c R n g}$, we identify $\operatorname{Mod}_{R}$ with ${ }_{R} \operatorname{Mod}_{R}$.

An $R$-module is called finitely projective if it is a retract of the $R$-module $R^{n}$ (with pointwise operations) for some natural number $n$. We denote the full subcategories of finitely projective modules by ${ }_{R} \mathrm{fpMod}, \mathrm{fpMod}_{R}$ and ${ }_{R} \mathrm{fpMod}_{S}$. We refer to [204] for a basic (bi)categorical account of modules.

2.1.2 Example The full subcategory of $\mathbf{c R n g}$ consisting of fields is denoted by Fld. For $K \in \mathbf{F l d}$, a $K$-module is better known as a $K$-vector space. In this case Vect $_{K}$ is just another name for $\operatorname{Mod}_{K}$. We abbreviate Vect $\mathbb{C}$ as Vect. A vector space is finitely projective as a module precisely when it is finite-dimensional as a vector space; we also denote fpMod $\mathrm{fu}_{K}$ as fdVect ${ }_{K}$.

An involutive field is a field $K$ that comes with a function $\ddagger: K \rightarrow K$ that satisfies $k^{\ddagger \ddagger}=k$ and commutes with addition and multiplication. Morphisms of involutive fields are field morphisms that in addition preserve the involution. These constitute a category denoted by InvFld.

A pre-Hilbert space over an involutive field $K$ is a $K$-vector space $X$ equipped with an inner product $\left\langle\left._{-}\right|_{-}\right\rangle: X \times X \rightarrow K$ satisfying

- $\langle x \mid k \cdot y\rangle=k \cdot\langle x \mid y\rangle$,

- $\langle x \mid y+z\rangle=\langle x \mid y\rangle+\langle x \mid z\rangle$,

- $\langle x \mid y\rangle=\langle y \mid x\rangle^{\ddagger}$,

- $\langle x \mid x\rangle=k^{\ddagger} \cdot k$ for some $k \in K$,

- $\langle x \mid x\rangle=0$ if and only if $x=0$.

We take morphisms of pre-Hilbert spaces to be the adjointable functions, i.e. those $f: X \rightarrow Y$ for which there exists a function $f^{\dagger}: Y \rightarrow X$ satisfying

$$
\langle f(x) \mid y\rangle_{Y}=\left\langle x \mid f^{\dagger}(y)\right\rangle_{X} .
$$

Such a function $f$ is automatically linear, and its so-called adjoint $f^{\dagger}$ is automatically unique. Thus we have a category preHilb ${ }_{K}$, and a full subcategory fdpreHilb $_{K}$ of finite dimensional pre-Hilbert spaces. We abbreviate preHilb $\mathbb{C}_{\mathbb{C}}$ by preHilb.

2.1.3 Example The inner product of a pre-Hilbert space canonically defines a norm by $\|x\|=\sqrt{\langle x \mid x\rangle}$, and hence a metric by $d(x, y)=\|x-y\|$. Let $K$ 
be either the reals $\mathbb{R}$, the complex numbers $\mathbb{C}$, or the quaternions $\mathbb{H}$. A preHilbert space over $K$ is called a Hilbert space when it is Cauchy-complete with regard to its canonical metric. A morphism of Hilbert spaces is a linear function that is furthermore continuous. The ensuing category is denoted by $\mathbf{H i l b}_{K}$. We abbreviate Hilb $\mathbf{H}_{\mathbb{C}}$ by Hilb. A linear function $f$ between Hilbert spaces is continuous if and only if it is bounded, in the sense that there is some $F \in K$ with $\|f(x)\| \leq F \cdot\|x\|$ 一 the infimum of such $F$ is then denoted by $\|f\|$. Any linear function between finite-dimensional Hilbert spaces is bounded and adjointable, and hence we also write $\mathbf{f d H i l b}_{K}$ for fdpreHilb $\mathbf{f l}_{K}$. Sometimes we will also restrict the morphisms of preHilb ${ }_{K}$ to just the bounded ones, getting a category preHilb bd .

2.1.4 Example The category PHilb of bounded lineair maps between Hilbert spaces up to global phase has the same objects as Hilb, but its homsets are quotiented by the action of the circle group $U(1)=\{z \in \mathbb{C} \mid\|z\|=1\}$. That is, continuous linear transformations $f, g: X \rightrightarrows Y$ are identified when $f(x)=$ $z \cdot g(x)$ for some $z \in U(1)$ and all $x \in X$. This gives a full functor $P:$ Hilb $\rightarrow$ PHilb.

2.1.5 Example We denote the category of (small) sets and functions by Set, and the category of (small) categories and functors by Cat.

2.1.6 Example Sets also form the objects of a different category, denoted by Rel. Here, a morphism from $X$ to $Y$ is a relation $R \subseteq X \times Y$. Composition of relations $R \subseteq X \times Y$ and $S \subseteq Y \times Z$ proceeds via the formula

$$
S \circ R=\left\{(x, z) \mid \exists_{y \in Y} .(x, y) \in R \text { and }(y, z) \in S\right\},
$$

and the identity relation on $X$ is the diagonal $\{(x, x) \mid x \in X\}$.

2.1.7 Example There is yet another choice of morphisms between sets as objects, namely the partial injections, forming a category PInj. A partial injection $X \rightarrow Y$ consists of a subset $\operatorname{dom}(f) \subseteq X$ and an injection $f: \operatorname{dom}(f) \rightarrow Y$. The composition of $f: \operatorname{dom}(f) \rightarrow Y$ and $g: \operatorname{dom}(g) \rightarrow Z$ with $\operatorname{dom}(g) \subseteq Y$ is given by composition of functions $g \circ f$, restricted to $\{x \in \operatorname{dom}(f) \mid f(x) \in \operatorname{dom}(g)\}$. Also, PInj can be regarded as a subcategory of Rel, since a relation $R \subseteq X \times Y$ can be regarded as (the graph of) a partial injection when for every $x \in X$ there is at most one $y \in Y$ with $(x, y) \in R$, and for every $y \in Y$ there is at most one $x \in X$ with $(x, y) \in R$. 


\subsection{Tensor products and monoids}

This section studies monoidal categories, monoids, and several relations between those notions.

2.2.1 Let us start by fixing notation. A monoidal category is a category $\mathbf{C}$ equipped with a bifunctor $\otimes: \mathbf{C} \times \mathbf{C} \rightarrow \mathbf{C}$, an object $I \in \mathbf{C}$, and natural isomorphisms $\lambda_{X}: I \otimes X \rightarrow X, \rho_{X}: X \otimes I \rightarrow X$ and $\alpha_{X, Y, Z}:(X \otimes Y) \otimes Z \rightarrow X \otimes(Y \otimes Z)$ that satisfy the familiar coherence equations (see [163]). We will often suppress coherence isomorphisms in diagrams and equations when no confusion can arise. A monoidal category is called strict when its coherence isomorphisms are identities; every monoidal category is monoidally equivalent to a strict one. It is symmetric when it furthermore comes with a natural isomorphism $\gamma_{X, Y}: X \otimes Y \rightarrow$ $Y \otimes X$ that satisfies $\gamma \circ \gamma=$ id and is compatible with the other coherence isomorphisms.

2.2.2 Example Common examples of (symmetric) monoidal structures are:

- Cartesian product $\times$ on the category Set, with any singleton $1=\{*\}$ as unit;

- Cartesian product $\times$ on the category Rel, with any singleton set as unit;

- product $\times$ on the category Cat, with the one-object category 1 as unit;

- disjoint union + on Set with the empty set as unit;

- disjoint union + on PInj, with the empty set as unit.

We will develop several more involved examples in this section.

2.2.3 A monoid could be seen as an internal version of a monoidal category. Its formulation requires a monoidal ambient category: a monoid consists of an object $M$, a morphism $\mu: M \otimes M \rightarrow M$ and a morphism $\eta: I \rightarrow M$ that satisfy the familiar unit and associativity equations:
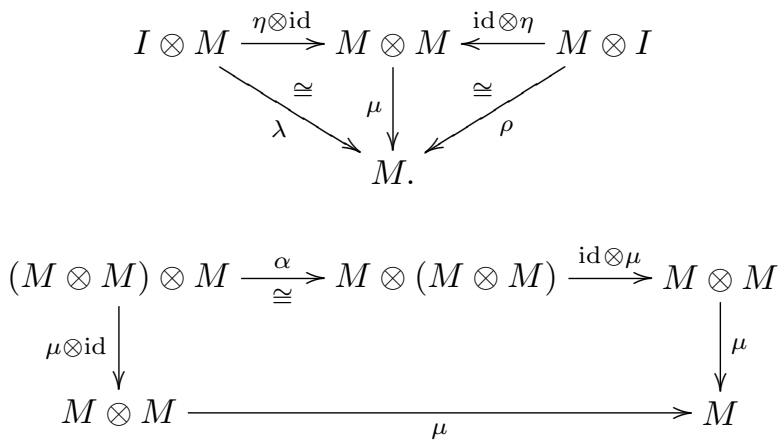
A monoid (in a symmetric monoidal category) is commutative when the following diagram commutes:

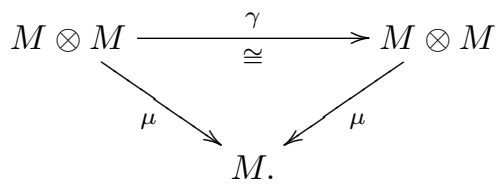

Dually, a comonoid $M$ in $\mathbf{C}$ is just a monoid in $\mathbf{C}^{\text {op }}$. We will denote its structure maps by $\nu: M \rightarrow I$ and $\delta: M \rightarrow M \otimes M$.

2.2.4 Example Monoids in a monoidal category $\mathbf{C}$ organise themselves into a category, denoted by $\operatorname{Mon}(\mathbf{C})$. A morphism $(M, \mu, \eta) \rightarrow\left(M^{\prime}, \mu^{\prime}, \eta^{\prime}\right)$ in this category is a morphism $f: M \rightarrow M^{\prime}$ in $\mathbf{C}$ that satisfies $f \circ \mu=\mu^{\prime} \circ(f \otimes f)$ and $f \circ \eta=\eta^{\prime}$. If $\mathbf{C}$ is symmetric monoidal, we denote the full subcategory of commutative monoids by $\mathbf{c M o n}(\mathbf{C})$. We abbreviate $\operatorname{Mon}($ Set $)$ and $\mathbf{c M o n}($ Set $)$ by Mon and cMon, respectively.

When $\mathbf{C}$ is symmetric monoidal, the category $\operatorname{cMon}(\mathbf{C})$ is again symmetric monoidal. The tensor product of monoids $(M, \mu, \eta)$ and $\left(M^{\prime}, \mu^{\prime}, \eta^{\prime}\right)$ has $M \otimes M^{\prime}$ as carrier object, with unit

$$
I \stackrel{\cong}{\longrightarrow} I \otimes I \stackrel{\eta \otimes \eta^{\prime}}{\longrightarrow} M \otimes M^{\prime},
$$

and multiplication

$$
\left(M \otimes M^{\prime}\right) \otimes\left(M \otimes M^{\prime}\right) \stackrel{\mathrm{id} \otimes \gamma \otimes \mathrm{id}}{\longrightarrow}(M \otimes M) \otimes\left(M^{\prime} \otimes M^{\prime}\right) \stackrel{\mu \otimes \mu^{\prime}}{\longrightarrow} M \otimes M^{\prime} .
$$

The monoidal unit $I$ of $\mathbf{C}$ becomes the monoidal unit in $\mathbf{c M o n}(\mathbf{C})$ when equipped with the unit id: $I \rightarrow I$ and multiplication $\lambda: I \otimes I \rightarrow I$. The coherence isomorphisms are inherited from $\mathbf{C}$.

2.2.5 Example A strict monoidal category is precisely a monoid in $(\mathbf{C a t}, \times, \mathbf{1})$. It is commutative as a monoid if and only if it is symmetric as a monoidal category. In fact, a monoidal category is precisely a so-called pseudo-monoid in $(\mathbf{C a t}, \times, \mathbf{1})$. However, we will refrain from using much 2-category theory in this thesis, and often restrict ourselves to strict monoidal structure.

Conversely, a monoid in Set is a strict monoidal category, when seen as the set of morphisms on one object with composition provided by the monoid multiplication. This one-object category is symmetric monoidal if and only if the monoid is commutative. 
2.2.6 The characterisation of a monoidal category as an object of Mon(Cat) lends itself for generalisation to enriched monoidal categories, as follows.

Let $\mathbf{V}$ be a symmetric monoidal category. We denote the category of $\mathbf{V}$ categories, and $\mathbf{V}$-functors between them by $\mathbf{V}$-Cat [141]. It is itself symmetric monoidal again [34, Proposition 6.2.9]; we describe its structure explicitly. For $\mathbf{V}$-categories $\mathbf{C}$ and $\mathbf{D}$, the objects of $\mathbf{C} \otimes \mathbf{D}$ are pairs $(X, Y)$ of objects $X$ of $\mathbf{C}$ and objects $Y$ of $\mathbf{D}$. The homobject $(\mathbf{C} \otimes \mathbf{D})\left((X, Y),\left(X^{\prime}, Y^{\prime}\right)\right)$ is $\mathbf{C}\left(X, X^{\prime}\right) \otimes$ $\mathbf{D}\left(Y, Y^{\prime}\right)$, where the tensor product is that of $\mathbf{V}$. Composition is given by

$$
\begin{aligned}
& (\mathbf{C} \otimes \mathbf{D})\left((X, Y),\left(X^{\prime}, Y^{\prime}\right)\right) \otimes(\mathbf{C} \otimes \mathbf{D})\left(\left(X^{\prime}, Y^{\prime}\right),\left(X^{\prime \prime}, Y^{\prime \prime}\right)\right) \\
& \mathbf{C}\left(X, X^{\prime}\right) \otimes \mathbf{D}\left(Y, Y^{\prime}\right) \otimes \mathbf{C}\left(X^{\prime}, X^{\prime \prime}\right) \otimes \mathbf{D}\left(Y^{\prime}, Y^{\prime \prime}\right) \\
& \downarrow \operatorname{id} \otimes \gamma \otimes \mathrm{id} \\
& \mathbf{C}\left(X, X^{\prime}\right) \otimes \mathbf{C}\left(X^{\prime}, X^{\prime \prime}\right) \otimes \mathbf{D}\left(Y, Y^{\prime}\right) \otimes \mathbf{D}\left(Y^{\prime}, Y^{\prime \prime}\right) \\
& \vee{ }^{\circ} \mathbf{C} \otimes{ }^{\circ} \mathrm{D} \\
& \mathbf{C}\left(X, X^{\prime \prime}\right) \otimes \mathbf{D}\left(Y, Y^{\prime \prime}\right) \\
& \downarrow \text { id } \\
& (\mathbf{C} \otimes \mathbf{D})\left((X, Y),\left(X^{\prime \prime}, Y^{\prime \prime}\right)\right),
\end{aligned}
$$

where ${ }^{\circ} \mathbf{C}$ and ${ }^{\circ} \mathbf{D}$ denote the composition of the $\mathbf{V}$-categories $\mathbf{C}$ and $\mathbf{D}$, respectively. Notice that this is really a special case of (2.2).

Hence it makes sense to speak of strict monoidal $\mathbf{V}$-categories as objects of $\operatorname{Mon}(\mathbf{V}$-Cat $)$. First of all, such a $\mathbf{C} \in \mathbf{M o n}(\mathbf{V}$-Cat $)$ is a $\mathbf{V}$-enriched category with objects $|\mathbf{C}|$, and hence comes equipped with $\mathbf{V}$-morphisms "identity" $i: I_{\mathbf{V}} \rightarrow \mathbf{C}(X, X)$ and "composition" ${ }^{\circ} \mathbf{C}: \mathbf{C}\left(X, X^{\prime}\right) \otimes_{\mathbf{V}} \mathbf{C}\left(X^{\prime}, X^{\prime \prime}\right) \rightarrow \mathbf{C}\left(X, X^{\prime \prime}\right)$. Furthermore, it means that there is a $\mathbf{V}$-functor $\otimes_{\mathbf{C}}$. Explicitly, we are given a morphism $\otimes_{\mathbf{C}}:|\mathbf{C}| \times|\mathbf{C}| \rightarrow|\mathbf{C}|$ in Set, and a morphism $\otimes_{\mathbf{C}}: \mathbf{C}\left(X, X^{\prime}\right) \otimes_{\mathbf{V}}$ $\mathbf{C}\left(Y, Y^{\prime}\right) \rightarrow \mathbf{C}\left(X \otimes_{\mathbf{C}} X^{\prime}, Y \otimes_{\mathbf{C}} Y^{\prime}\right)$ in $\mathbf{V}$. Finally, it means we are given an object $I_{\mathbf{C}} \in|\mathbf{C}|$. These data satisfy the (strict) monoid requirements, like $I_{\mathbf{C}} \otimes_{\mathbf{C}} X=X$.

Analogously, an enriched symmetric monoidal category is defined as an object of $\mathbf{c M o n}\left(\mathbf{V}\right.$-Cat), and as such in addition satisfies $X \otimes_{\mathbf{C}} Y=Y \otimes_{\mathbf{C}} X$.

2.2.7 A left-action of a monoid $M$ on an object $X$ of a monoidal category $\mathbf{C}$ ) is a morphism $\bullet: M \otimes X \rightarrow X$ that is compatible with the tensor product of $\mathbf{C}$, in the sense that the following diagram commutes:

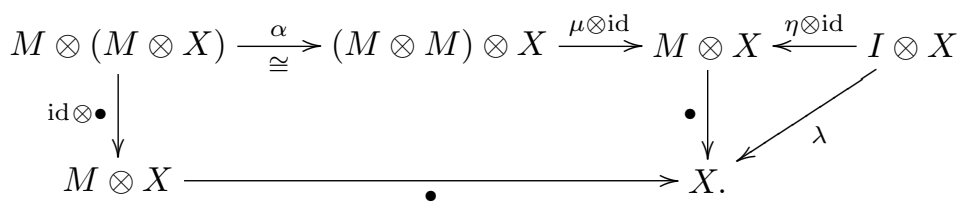


A right-action is defined similarly; if $\mathbf{C}$ is symmetric monoidal, every left-action corresponds uniquely to a right-action, and we simply speak of an action. A morphism $(X, \bullet) \rightarrow\left(X^{\prime}, \bullet^{\prime}\right)$ of actions of $M$ is an morphism $f: X \rightarrow X^{\prime}$ of $\mathbf{C}$ such that $\bullet^{\prime} \circ(\operatorname{id} \otimes f)=f \circ \bullet$. Thus, we get categories ${ }_{M} \operatorname{Act}(\mathbf{C})$ of left-actions of $M$ and $\mathbf{A c t}_{M}(\mathbf{C})$ of right-actions. When $\mathbf{C}=\mathbf{S e t}$, we abbreviate them to ${ }_{M} \mathbf{A c t}$ and $\mathbf{A c t}_{M}$, respectively. There is an obvious forgetful functor $\operatorname{Act}_{M}(\mathbf{C}) \rightarrow \mathbf{C}$. (See also [163, Section VII.4].)

Thus, for $R \in \mathbf{R n g}$, we can restate Example 2.1.1 as $\operatorname{Mod}_{R}=\operatorname{Act}_{R}(\mathbf{A b})$, where $\mathbf{A b}$ is the category of Abelian groups.

2.2.8 Example Continuing Example 2.2.5, we notice that the endomorphisms $\mathbf{C}(X, X)$ on any object $X$ in a category $\mathbf{C}$ form a monoid (in Set). We will pay special attention to the monoid $\mathbf{C}(I, I)$, whose elements we call scalars, because this monoid comes with an action on homsets called scalar multiplication. This action $\bullet: \mathbf{C}(I, I) \times \mathbf{C}(X, Y) \rightarrow \mathbf{C}(X, Y)$ is defined as the function that sends a pair consisting of a scalar $s: I \rightarrow I$ and any morphism $f: X \rightarrow Y$ to the composite

$$
X \stackrel{\cong}{\longrightarrow} I \otimes X \stackrel{s \otimes f}{\longrightarrow} I \otimes Y \stackrel{\cong}{\longrightarrow} Y .
$$

To see that this indeed defines an action, one readily verifies that id $\bullet f=f$ and $r \bullet(s \bullet f)=(r \circ s) \bullet f$.

The name "scalar multiplication" is explained by the fact that the scalars in ${ }_{R}$ Mod are in bijective correspondence with elements of $R$, so that $r \bullet f$ is really pointwise scalar multiplication (on the left). The following lemma shows that many of the familiar properties of pointwise scalar multiplication are retained in any monoidal category.

2.2.9 Lemma For scalars $r, s \in \mathbf{C}(I, I)$ and morphisms $f, g$ in a monoidal category:

(a) $s$ induces a natural transformation $\operatorname{Id}_{\mathbf{C}} \Rightarrow \operatorname{Id}_{\mathbf{C}}$ with component $s \bullet \operatorname{id}_{X}$ at $X$;

(b) $r \bullet s=r \circ s$;

(c) $(r \bullet f) \circ(s \bullet g)=(r \circ s) \bullet(f \circ g)$;

(d) $(r \bullet f) \otimes(s \bullet g)=(r \circ s) \bullet(f \otimes g)$.

Proof See [77, Lemma 2.33] and [77, Corollary 2.34].

2.2.10 $I$ is the monoidal unit of $\mathbf{C}$ with $\otimes$, and analogously Id: $\mathbf{C} \rightarrow \mathbf{C}$ is the monoidal unit of the functor category $[\mathbf{C}, \mathbf{C}]$ with composition as tensor product. As a special case of 2.2 .5 , the set $\mathrm{Nat}\left(\mathrm{Id}_{\mathbf{C}}, \mathrm{Id}_{\mathbf{C}}\right)$ of natural transformations $\mathrm{Id}_{\mathbf{C}} \Rightarrow \operatorname{Id}_{\mathbf{C}}$ is a monoid under composition. If we temporarily denote the natural 
transformation from (a) of the previous lemma by $\widehat{\left(\_\right)}: \operatorname{Id}_{\mathbf{C}} \Rightarrow \operatorname{Id}_{\mathbf{C}}$, the assignment $s \mapsto \widehat{s}$ is a monoid morphism $\mathbf{C}(I, I) \rightarrow \mathbf{N a t}\left(\operatorname{Id}_{\mathbf{C}}, \operatorname{Id}_{\mathbf{C}}\right)$, since one easily verifies that $\widehat{\mathrm{d}}=\mathrm{id}$ and $\widehat{r \bullet s}=\widehat{r} \circ \widehat{s}$.

The scalars in a symmetric monoidal category are always commutative [142], which the following lemma proves directly.

2.2.11 Lemma If $\mathbf{C}$ is a symmetric monoidal category, $\mathbf{C}(I, I)$ is a commutative monoid. Then $\mathbf{C} \mapsto \mathbf{C}(I, I)$ extends to a functor $\mathbf{c M o n}(\mathbf{V}$-Cat $) \rightarrow \mathbf{c M o n}(\mathbf{V})$.

PROOF The following diagram establishes commutativity directly, and easily carries over to the enriched case:

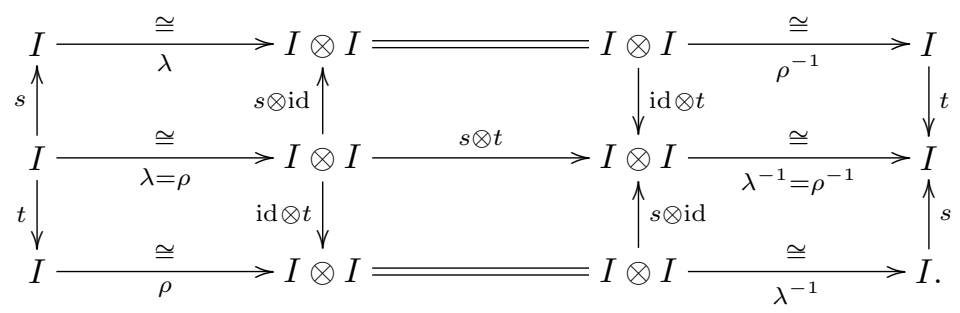

Notice how this essentially uses the coherence property $\lambda_{I}=\rho_{I}$ [133].

We will take a further look at scalars and scalar multiplication in Sections 2.4 and 3.6. For now, we spend some time developing the familiar tensor product of vector spaces as a specific instance of a general construction due to Anders Kock and Brian Day ([144], but see also [124]).

2.2.12 A monad on a category $\mathbf{C}$ is precisely a monoid in the category $[\mathbf{C}, \mathbf{C}]$ of endofunctors on $\mathbf{C}$ and natural transformations between them (with composition as tensor). Recall that an endofunctor $T$ on a symmetric monoidal category is strong if there is a "strength" natural transformation st: $X \otimes T Y \rightarrow T(X \otimes Y)$ satisfying suitable coherence conditions [145]. In particular, a monad is strong when strength is furthermore compatible with the monad structure [124]. This can be formulated succinctly: a strong monad on $\mathbf{C}$ is precisely a monoid in the category of strong functors $\mathbf{C} \rightarrow \mathbf{C}$, with natural transformations that commute with strength between them (see also [115]).

The strength map and its symmetric dual st ${ }^{\prime}=T(\gamma) \circ$ st $\circ \gamma: T X \otimes Y \rightarrow$ $T(X \otimes Y)$ can be combined in two ways as maps $T X \otimes T Y \rightrightarrows T(X \otimes Y)$ :

$$
\begin{aligned}
\mathrm{dst} & =\mu \circ T\left(\mathrm{st}^{\prime}\right) \circ \mathrm{st}, \\
\mathrm{dst}^{\prime} & =\mu \circ T(\mathrm{st}) \circ \mathrm{st}^{\prime} .
\end{aligned}
$$

The monad $T$ is called commutative if these "double strength" maps coincide. 
2.2.13 Definition We are now in a position to define the Kock-Day tensor product. Let $T$ be a commutative monad on $\mathbf{V}$, and suppose that its category of (Eilenberg-Moore) algebras $\operatorname{Alg}(T)$ has coequalisers of reflexive pairs-recall that a pair of parallel morphisms $f, g: X \rightrightarrows Y$ is called reflexive when there is a common right inverse, i.e. a morphism $h: Y \rightarrow X$ satisfying $f \circ h=\operatorname{id}_{Y}=g \circ h$. For algebras $\varphi: T X \rightarrow X$ and $\psi: T Y \rightarrow Y$, define $\varphi \otimes \psi$ as the coequaliser

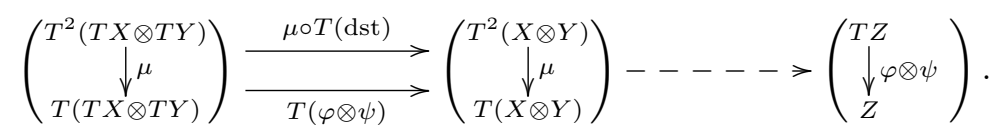

If we furthermore define $I_{\mathrm{Alg}(T)}$ as the free algebra $\mu: T^{2}\left(I_{\mathbf{V}}\right) \rightarrow T\left(I_{\mathbf{V}}\right)$, we obtain a symmetric monoidal structure on $\operatorname{Alg}(T)$. Moreover, the free functor $\mathbf{V} \rightarrow \operatorname{Alg}(T)$ preserves monoidal structure [124, Lemma 5.2].

The following lemma provides the monad to use in our situation.

2.2.14 Lemma If $M$ is a monoid in a monoidal category $\mathbf{V}$, then $M \otimes\left(\_\right): \mathbf{V} \rightarrow \mathbf{V}$ is a monad, whose category of algebras is $\mathbf{A c t}_{M}(\mathbf{V})$. The monad $M \otimes\left(\_\right)$is strong if $\mathbf{V}$ is symmetric monoidal, and it is commutative if and only if the monoid $M$ is.

Proof The unit and multiplication of the monad are given by

$$
\begin{aligned}
& \eta: X \stackrel{\cong}{\rightarrow} I \otimes X \stackrel{e \otimes \mathrm{id}}{\longrightarrow} M \otimes X, \\
& \mu: M \otimes(M \otimes X) \stackrel{\cong}{\longrightarrow}(M \otimes M) \otimes X \stackrel{m \otimes \mathrm{id}}{\longrightarrow} M \otimes X,
\end{aligned}
$$

where we write $e$ and $m$ for the structure maps of the monoid $M$. If $\mathbf{C}$ is a symmetric monoidal category, then there is a strength map

$$
\text { st }: X \otimes(M \otimes Y) \cong(X \otimes M) \otimes Y \stackrel{\gamma \otimes \mathrm{id}}{\longrightarrow}(M \otimes X) \otimes Y \cong M \otimes(X \otimes Y) .
$$

The double strength maps boil down to

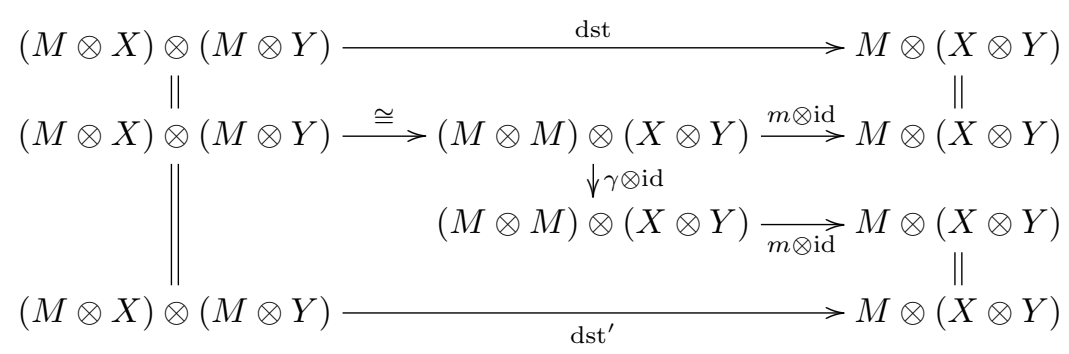

Hence they coincide if and only if the monoid $M$ is commutative. 
2.2.15 Definition Let $M$ be a monoid in a monoidal category $\mathbf{V}$. We say that $\mathbf{V}$ is suitable for $M$ when $\mathbf{A c t}_{M}(\mathbf{V})$ has coequalisers of reflexive pairs. We call $\mathbf{V}$ suitable when it is suitable for any monoid in it.

This is precisely what is needed to facilitate the construction of the KockDay tensor product on $\mathbf{A c t}_{M}(\mathbf{V})$. A common scenario in which this criterion is fulfilled is when $\mathbf{V}$ has coequalisers of reflexive pairs and $M \otimes\left(\_\right)$has a right adjoint.

2.2.16 Example An exemplary case is $\mathbf{V}=$ Set. This category is suitable for any monoid $M$ in it, since $\operatorname{Act}_{M}(\mathbf{S e t})$ is in fact a topos (see Chapter 5). For $X, Y \in \mathbf{A c t}_{M}(\mathbf{S e t})$, the Kock-Day tensor product $X \otimes Y$ is given explicitly by $X \times Y / \sim$, where $\sim$ is the (least) equivalence relation determined by $(m \bullet x, y) \sim$ $(x, m \bullet y)$, with action given by $m \bullet[x, y]=[m \bullet x, y]=[x, m \bullet y]$.

Thus, morphisms $X \otimes Y \rightarrow Z$ correspond to functions $X \times Y \rightarrow Z$ that are $M$-equivariant in both variables separately. This is a general feature of the KockDay tensor product, which the following example works out through comparison with the special case of vector spaces.

2.2.17 Example Consider (complex) vector spaces $X, Y, Z$, i.e. objects in $\operatorname{Mod}_{\mathbb{C}}$. Recall that a linear function $f: X \times Y \rightarrow Z$, i.e. a morphism in $\operatorname{Mod}_{\mathbb{C}}$, is called bilinear when it is linear in each of its variables separately. The familiar tensor product $X \otimes Y$ of vector spaces is the unique one such that linear functions $X \otimes Y \rightarrow Z$ correspond to bilinear functions $X \times Y \rightarrow Z$.

Now, $T(X)=\{\varphi: X \rightarrow \mathbb{C} \mid \operatorname{supp}(\varphi)$ finite $\}$ defines a commutative monad that we will study in 2.5.3, where the $\operatorname{support}$ is $\operatorname{supp}(\varphi)=\{i \in I \mid \varphi(i) \neq 0\}$. Identifying $X, Y$ and $Z$ with algebras $\varphi: T X \rightarrow X, \psi: T Y \rightarrow Y$ and $\theta: T Z \rightarrow Z$, we can characterise bilinear functions as follows. A morphism $f: X \otimes Y \rightarrow Z$ is bilinear if and only if

$$
\theta \circ T f \circ \mathrm{dst}=f \circ(\varphi \otimes \psi) .
$$

We call a morphism satisfying this equation in any category of algebras of a commutative monad a bimorphism $[\varphi, \psi] \rightarrow \theta$.

With this characterisation we can formulate the universal property of the tensor product of vector spaces in any category of algebras of a commutative monad. We say that a monoidal structure $\otimes$ on $\operatorname{Alg}(T)$ is universal for bimorphisms when for each pair of algebras $\varphi, \psi$, there is a bimorphism $[\varphi, \psi] \rightarrow \varphi \otimes \psi$ through which any bimorphism $[\varphi, \psi] \rightarrow \theta$ factorises uniquely. As a special case, the familiar tensor product of vector spaces is universal for bimorphisms.

The Kock-Day tensor product is universal for bimorphisms [124, Lemma 5.1].

2.2.18 Example The category cMon of commutative monoids is the category of (Eilenberg-Moore) algebras for the commutative monad $\widehat{\mathbb{N}}$ : Set $\rightarrow$ Set given 
by

$$
\widehat{\mathbb{N}}(X)=\{\varphi: X \rightarrow \mathbb{N} \mid \operatorname{supp}(\varphi) \text { is finite }\} .
$$

We will discuss this monad more thoroughly in 2.5.3. Hence, as a special case of the Kock-Day tensor product, we get a monoidal structure on cMon that is universal for bimorphisms. This monoidal structure is in general different from that inhereted from Set as discussed in 2.2.4. For example, the unit for this monoidal structure is $(\mathbb{N},+, 0)$, the free commutative monoid on 1 .

2.2.19 Example Whereas the category Hilb of Hilbert spaces has two symmetric monoidal structures, namely the Cartesian product $\oplus$ (see also Section 2.3) and the tensor product $\otimes$, only the latter descends to the category PHilb. For example, for morphisms $f, g: X \rightrightarrows Y$ of Hilb, if we define $f^{\prime}=i \cdot f$ and $g^{\prime}=-g$, then $f \sim f^{\prime}$ and $g \sim g^{\prime}$. But

$$
\left(f^{\prime} \oplus g^{\prime}\right)(x, y)=(i \cdot f(x),-g(y)) \neq(u \cdot f(x), u \cdot g(y))=(u \cdot(f \oplus g))(x, y)
$$

for any $u \in U(1)$, so $f \oplus g \nsim f^{\prime} \oplus g^{\prime}$. Hence the Cartesian product $\oplus$ of Hilb does not give a well-defined monoidal structure on PHilb.

On the other hand, the tensor product $\otimes$ on Hilb is universal for bimorphisms, so it does induce a well-defined monoidal structure on PHilb. For if $f \sim f^{\prime}$ and $g \sim g^{\prime}$, say $f=u \cdot f^{\prime}$ and $g=v \cdot g^{\prime}$ for $u, v \in U(1)$, then

$$
f \otimes g=\left(u \cdot f^{\prime}\right) \otimes\left(v \cdot g^{\prime}\right)=u \cdot v \cdot\left(f^{\prime} \otimes g^{\prime}\right)
$$

whence $f \otimes g \sim f^{\prime} \otimes g^{\prime}$.

We finish this section with the following argument, due to Peter Hilton and Beno Eckmann, who proved it for $\mathbf{C}=$ Set ([78], but see also [163, Exercise II.5.5]). It states that when an object carries two monoid structures and the multiplication map of one is a monoid homomorphism with respect to the other, then the two monoid structures coincide and are in fact commutative.

2.2.20 Lemma (Hilton-Eckmann) Let $X$ be an object in a symmetric monoidal category $\mathbf{C}$, and let $\mu_{1}, \mu_{2}: X \otimes X \rightrightarrows X$ and $\eta_{1}, \eta_{2}: I \rightrightarrows X$ be morphisms. If $\left(X, \mu_{1}, \eta_{1}\right)$ and $\left(X, \mu_{2}, \eta_{2}\right)$ are both monoids and the following diagram commutes,

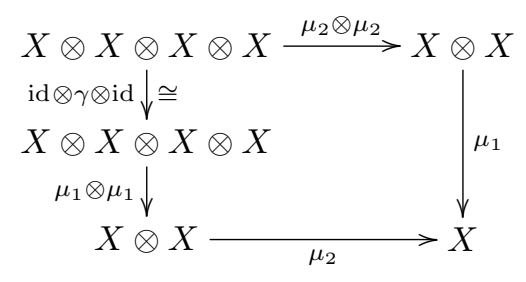


then $\left(X, \mu_{1}, \eta_{1}\right)=\left(X, \mu_{2}, \eta_{2}\right)$ is in fact a commutative monoid.

PROOF First we show that $\eta_{1}=\eta_{2}$.

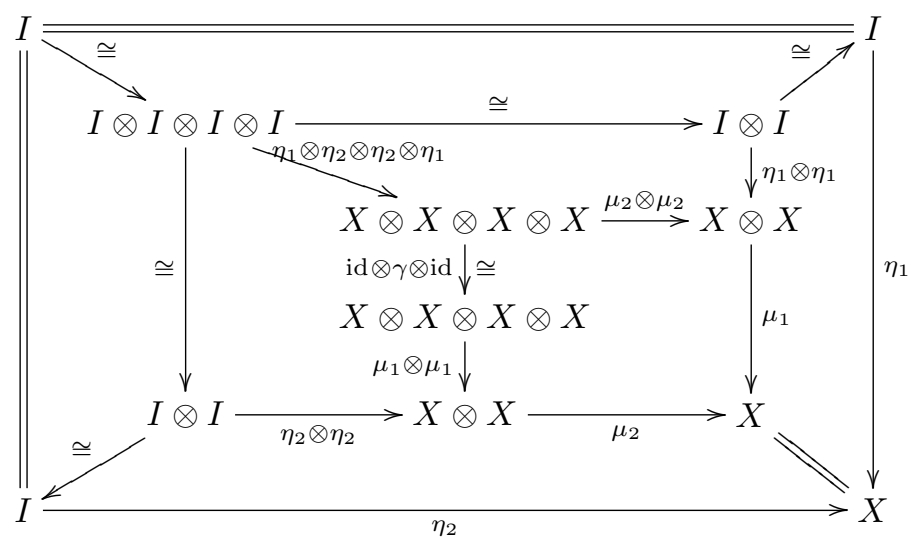

To prevent a forest of diagrams, we give the rest of the proof for $\mathbf{C}=$ Set (as in [78]). The reader can check for herself that it generalises to any symmetric monoidal category. Let us further temporarily abbreviate $\eta_{1}=\eta_{2}$ to $1, \mu_{1}(x, y)$ to $x \cdot y$, and $\mu_{2}(x, y)$ to $x \odot y$.

$$
\begin{aligned}
x \cdot y & =(1 \odot x) \cdot(y \odot 1) \stackrel{(2.3)}{=}(1 \cdot y) \odot(x \cdot 1)=y \odot x \\
& =(y \cdot 1) \odot(1 \cdot x) \stackrel{2.3}{=}(y \odot 1) \cdot(1 \odot x)=y \cdot x .
\end{aligned}
$$

Notice that this provides an alternative proof of Lemma 2.2.11; it follows from Lemma 2.2.9(b) that the scalars are a monoid under both $\bullet$ and $\circ$. Since Lemma 2.2.9(c) means that diagram (2.3) commutes, the result follows from Lemma 2.2.20,

\subsection{Biproducts}

This section considers a special kind of monoidal structure called biproduct.

2.3.1 As already noted in the previous section, (finite) products and coproducts are particular instances of monoidal structure on a category. A coproduct, for example, is a monoidal product that in addition is the vertex of a universal cocone. We denote the legs of this universal cocone, the coprojections, by $\kappa$. For example, we write $X_{1} \stackrel{\kappa_{1}}{\longrightarrow} X_{1}+X_{2} \stackrel{\kappa_{2}}{\longleftarrow} X_{2}$, or $X \stackrel{\kappa_{X}}{\longrightarrow} X+Y \stackrel{\kappa_{Y}}{\longleftarrow} Y$, or even $X \stackrel{\kappa}{\longrightarrow} X+X^{\prime} \stackrel{\kappa^{\prime}}{\longleftarrow} X^{\prime}$. The unit object 0 of the monoidal structure is 
initial, i.e. there is a unique morphism $0 \rightarrow X$ for every object $X$. We denote the codiagonal map, i.e. the cotuple [id, id] : $X+X \rightarrow X$, by $\nabla$.

Likewise, a product $X_{1} \times X_{2}$ comes with projections that we denote by $\pi$, as in $X_{1} \stackrel{\pi_{1}}{\longleftarrow} X_{1} \times X_{2} \stackrel{\pi_{2}}{\longrightarrow} X_{2}$. The unit object 1 of the monoidal structure is terminal, i.e. there is a unique morphism $X \rightarrow 1$ for every object $X$. We denote the diagonal map, i.e. the tuple $\langle$ id, id $\rangle: X \rightarrow X \times X$, by $\Delta$.

The following theorem characterises algebraically when a monoidal product is a coproduct, without any reference to universal properties, in a way reminiscent of [87] (see also [84]).

2.3.2 Theorem A symmetric monoidal structure $(\oplus, 0)$ on a category $\mathbf{C}$ provides finite coproducts if and only if the forgetful functor $\mathbf{c M o n}(\mathbf{C}) \rightarrow \mathbf{C}$ is an isomorphism of categories.

ProOF Suppose that $(\oplus, 0)$ provides finite coproducts, with the coherence maps $\alpha, \lambda$ and $\rho$ induced by the coproducts. Denote the forgetful functor $\mathbf{c M o n}(\mathbf{C}) \rightarrow$ $\mathbf{C}$ by $U$, and define $F: \mathbf{C} \rightarrow \mathbf{c M o n}(\mathbf{C})$ on objects as $F(X)=(X, \nabla, u)$, where $\nabla=\left[\operatorname{id}_{X}, \operatorname{id}_{X}\right]: X \oplus X \rightarrow X$, and $u$ is the unique morphism $0 \rightarrow X$. On a morphism $f$, it acts as $F(f)=f$. Then trivially $U \circ F=\mathrm{Id}$. To prove that also $F \circ U=\mathrm{Id}$, we show that there can be only one (commutative) monoid structure on $X \in \mathbf{C}$ with respect to $(\oplus, 0)$, i.e. for any $(X, \mu, \eta) \in \mathbf{c M o n}(\mathbf{C})$ one has $\mu=[\mathrm{id}, \mathrm{id}]$. This suffices because $\eta$ is necessarily the unique morphism $0 \rightarrow X$. We have

$$
\mu \circ \kappa_{1}=\mu \circ\left[\kappa_{1}, \kappa_{2} \circ u\right] \circ \kappa_{1}=\mu \circ(\operatorname{id} \oplus u) \circ \kappa_{1}=\rho \circ \kappa_{1}=\mathrm{id},
$$

since $\kappa_{1}: X \rightarrow X \oplus 0$ equals the coherence isomorphism $\rho^{-1}$. Likewise $\mu \circ \kappa_{2}=$ id, so $\mu=$ [id, id], as needed.

Conversely, suppose that $\mathbf{c M o n}(\mathbf{C}) \underset{F}{\stackrel{U}{\underset{ }{\gtrless}}} \mathbf{C}$ is an isomorphism. By definition $U(X, \mu, \eta)=X$, so the monoid $F(X)$ is carried by $X$. Since $F$ is a functor, the monoid structure maps, say $\nabla_{X}: X \oplus X \rightarrow X$ and $u_{X}: 0 \rightarrow X$, are natural in $X$. We first prove that 0 is an initial object. We have that $\left(0, \nabla_{0}, u_{0}\right)$ and $\left(0, \lambda_{0}, i_{0}\right)$ are both monoids (in $\left.\mathbf{C}\right)$. Moreover, they satisfy the Hilton-Eckmann condition (2.3), so by Lemma 2.2.20 we have $u_{0}=\mathrm{id}_{0}$. Naturality of $u$ yields

$$
f=f \circ \operatorname{id}_{0}=f \circ u_{0}=u_{X}
$$

for any $f: 0 \rightarrow X$. Hence $u_{X}$ is the unique morphism $0 \rightarrow X$, and 0 is indeed an initial object. Finally, we show that $X \oplus Y$ is a coproduct of $X$ and $Y$. Define $\kappa_{X}: X \stackrel{\rho^{-1}}{\longrightarrow} X \oplus 0 \stackrel{\operatorname{id} \oplus u_{Y}}{\longrightarrow} X \oplus Y$ and $\kappa_{Y}: Y \stackrel{\lambda^{-1}}{\longrightarrow} 0 \oplus Y \stackrel{u_{X} \oplus \text { id }}{\longrightarrow} X \oplus Y$ as 
coprojections. Put $[f, g]=\nabla_{Z} \circ(f \oplus g): X \oplus Y \rightarrow Z$ for given $f: X \rightarrow Z$ and $g: Y \rightarrow Z$. Then

$$
\begin{aligned}
{[f, g] \circ \kappa_{X} } & =\nabla_{Z} \circ(f \oplus g) \circ\left(\mathrm{id} \oplus u_{Y}\right) \circ \rho \\
& =\nabla_{Z} \circ\left(\mathrm{id} \oplus\left(g \circ u_{Y}\right)\right) \circ(f \oplus \mathrm{id}) \circ \rho \\
& =\nabla_{Z} \circ\left(\mathrm{id} \oplus u_{Y}\right) \circ(f \oplus \mathrm{id}) \circ \rho \\
& =\rho \circ(f \oplus \mathrm{id}) \circ \rho^{-1}=f .
\end{aligned}
$$

$u$ natural

$\nabla, u$ monoid

$\rho$ natural

Analogously, $[f, g] \circ \kappa_{Y}=g$. Moreover, $[f, g]$ is the unique such map since

$$
\begin{aligned}
{\left[\kappa_{X}, \kappa_{Y}\right]=} & \left(\nabla_{X} \oplus \nabla_{Y}\right) \circ(\mathrm{id} \oplus \gamma \oplus \mathrm{id}) \circ\left(\mathrm{id} \oplus u_{Y} \oplus \mathrm{id}\right) \\
& \circ\left(\rho^{-1} \oplus \mathrm{id}\right) \circ\left(\mathrm{id} \oplus u_{X} \oplus \mathrm{id}\right) \circ\left(\mathrm{id} \oplus \lambda^{-1}\right) \\
= & \left(\nabla_{X} \oplus \mathrm{id}\right) \circ\left(\mathrm{id} \oplus \nabla_{Y}\right) \circ\left(\mathrm{id} \oplus u_{Y} \oplus \mathrm{id}\right) \circ(\mathrm{id} \oplus \gamma \oplus \mathrm{id}) \\
& \circ\left(\rho^{-1} \oplus \mathrm{id}\right) \circ\left(\mathrm{id} \oplus u_{X} \oplus \mathrm{id}\right) \circ\left(\mathrm{id} \oplus \lambda^{-1}\right) \\
= & \left(\nabla_{X} \oplus \mathrm{id}\right) \circ(\mathrm{id} \oplus \lambda) \circ(\mathrm{id} \oplus \gamma \oplus \mathrm{id}) \\
& \circ\left(\rho^{-1} \oplus \mathrm{id}\right) \circ\left(\mathrm{id} \oplus u_{X} \oplus \mathrm{id}\right) \circ\left(\mathrm{id} \oplus \lambda^{-1}\right) \\
= & \left(\nabla_{X} \oplus \mathrm{id}\right) \circ\left(\mathrm{id} \oplus u_{X} \oplus \mathrm{id}\right) \circ\left(\mathrm{id} \oplus \lambda^{-1}\right) \\
= & (\mathrm{id} \oplus \lambda) \circ\left(\mathrm{id} \oplus \lambda^{-1}\right)=\mathrm{id} .
\end{aligned}
$$

Dually, a symmetric monoidal structure $(\oplus, 0)$ on a category $\mathbf{C}$ provides finite products if and only if $\mathbf{C}^{\mathrm{op}}$ is isomorphic to the category of commutative comonoids in $\mathbf{C}$.

2.3.3 A zero object is an object 0 that is simultaneously initial and terminal. That is, there are unique maps $X \rightarrow 0$ and $0 \rightarrow X$ for any object $X$, which we denote by $0_{X, 0}$ and $0_{0, X}$. These are automatically epi and mono, respectively. For any pair of objects $X, Y$, there is a unique zero morphism $0_{X, Y}: X \stackrel{0_{X, 0}}{\longrightarrow} 0 \stackrel{0_{0, Y}}{\longrightarrow} Y$. If no confusion can arise, we abbreviate zero morphisms simply as $X \stackrel{0}{\longrightarrow} Y$.

2.3.4 A biproduct of $X_{1}$ and $X_{2}$ is an object $X_{1} \oplus X_{2}$ that is simultaneously a product with projections $\pi_{i}: X_{1} \oplus X_{2} \rightarrow X_{i}$ and a coproduct with coprojections $\kappa_{i}: X_{i} \rightarrow X_{1} \oplus X_{2}$, such that

$$
\begin{aligned}
& \pi_{i} \circ \kappa_{i}=\mathrm{id}, \\
& \pi_{i} \circ \kappa_{j}=0, \quad \text { for } i \neq j .
\end{aligned}
$$

A category with a chosen zero object and a chosen biproduct for each pair of objects is said to have finite biproducts. Hence finite biproducts are a special kind of symmetric monoidal structure on a category. Indeed, equations 2.4 and (2.5) ensure that a choice of biproduct for each pair of objects canonically 
extends to a bifunctor by $f_{1} \oplus f_{2}=f_{1}+f_{2}=f_{1} \times f_{2}: X_{1} \oplus X_{2} \rightarrow Y_{1} \oplus Y_{2}$ for $f_{i}: X_{i} \rightarrow Y_{i}$, where $f_{1}+f_{2}$ is defined as usual as $\left[\kappa_{1} \circ f_{1}, \kappa_{2} \circ f_{2}\right]$, and $f_{1} \times f_{2}=\left\langle f_{1} \circ \pi_{1}, f_{2} \circ \pi_{2}\right\rangle$. For this it suffices that $\left\langle f_{1} \circ \pi_{1}, f_{2} \circ \pi_{2}\right\rangle \circ \kappa_{i}=\kappa_{i} \circ f_{i}$, as this is the defining property of $f_{1}+f_{2}$. By equations (2.4) and (2.5) we get $\left\langle f_{1} \circ \pi_{1}, f_{2} \circ \pi_{2}\right\rangle \circ \kappa_{1}=\left\langle f_{1} \circ \pi_{1} \circ \kappa_{1}, f_{2} \circ \pi_{2} \circ \kappa_{1}\right\rangle=\left\langle f_{1}, 0\right\rangle$. Since $\pi_{i} \circ\left\langle f_{1}, 0\right\rangle=$ $\pi_{i} \circ \kappa_{1} \circ f_{1}$ and projections are jointly mono, indeed $\left\langle f_{1} \circ \pi_{1}, f_{2} \circ \pi_{2}\right\rangle \circ \kappa_{i}=\kappa_{i} \circ f_{i}$ for $i=1$. The case $i=2$ is analogous.

Like products and coproducts, finite biproducts can be characterised algebraically, without reference to universal properties.

2.3.5 Definition For a category $\mathbf{C}$, define $\mathbf{C}_{\leftrightarrows}$ as the full subcategory of $\mathbf{C}^{o p} \times \mathbf{C}$ consisting of objects $(X, X)$. Identifying its objects with those of $\mathbf{C}$, a morphism $f: X \rightarrow Y$ in $\mathbf{C}_{\leftrightarrows}$ is a pair of morphisms $f_{\leftarrow}: Y \rightarrow X, f_{\rightarrow}: X \rightarrow Y$ of $\mathbf{C}$. This construction works equally well for $\mathrm{V}$-enriched categories.

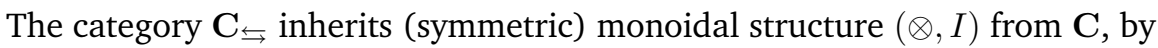
$\left(f_{\leftarrow}, f_{\rightarrow}\right) \otimes\left(g_{\leftarrow}, g_{\rightarrow}\right)=\left(f_{\leftarrow} \otimes g_{\leftarrow}, f_{\rightarrow} \otimes g_{\rightarrow}\right)$. Hence an object in $\mathbf{c M o n}\left(\mathbf{C}_{\leftrightarrows}\right)$ consists of a commutative monoid and a commutative comonoid in $\mathbf{C}$ carried by the same object.

2.3.6 Corollary A symmetric monoidal structure $(\oplus, 0)$ on a category $\mathbf{C}$ provides finite biproducts if and only if the forgetful functor $\mathbf{c M o n}\left(\mathbf{C}_{\leftrightarrows}\right) \rightarrow \mathbf{C}_{\leftrightarrows}$ is an isomorphism of categories.

PROOF Suppose the underlying functor is an isomorphism. Then 0 is an initial object by Theorem 2.3.2, and a terminal object by the dual of that theorem. Hence 0 is a zero object. To verify equation (2.4), we abuse the notation of Theorem 2.3.2 by $n_{i}=\left(u_{X_{i}}\right)_{\leftarrow}: X_{i} \rightarrow 0$ and $u_{i}=\left(u_{X_{i}}\right)_{\rightarrow}: 0 \rightarrow X_{i}$ for $i \in\{1,2\}$, and put $j \in\{1,2\}$ such that $i \neq j$ :

$$
\pi_{i} \circ \kappa_{i}=\rho \circ\left(\operatorname{id} \oplus n_{j}\right) \circ\left(\operatorname{id} \oplus u_{j}\right) \circ \rho^{-1}=\rho \circ\left(\operatorname{id} \oplus u_{j}\right) \circ \rho^{-1}=\mathrm{id} .
$$

Also, (2.5) holds, since for $i \neq j$ we have

$$
\begin{aligned}
\pi_{2} \circ \kappa_{1} & =\lambda \circ\left(n_{1} \oplus \mathrm{id}\right) \circ\left(\mathrm{id} \oplus u_{2}\right) \circ \rho^{-1} \\
& =\lambda \circ\left(\mathrm{id} \oplus u_{2}\right) \circ\left(n_{1} \oplus \mathrm{id}\right) \circ \rho^{-1} \\
& =0 .
\end{aligned}
$$

The converse is trivial given Theorem 2.3.2 and its dual: if $\mathbf{C}$ has coinciding finite products and coproducts, then the forgetful functor is an isomorphism.

2.3.7 The previous corollary enables us to talk about finite biproducts in $\mathbf{V}$ enriched categories without having to resort to a product structure on $\mathbf{V}$ or to 
weighted (co)limits. In particular, it is more general than the usual notion of a $\mathbf{V}$-coproduct, which requires $\mathbf{V}$ to have finite products - if $\mathbf{V}$ is a category with finite products, then a finite coproduct structure on $\mathbf{C} \in \mathbf{V}$-Cat is traditionally regarded as $\mathbf{V}$-natural isomorphisms $\mathbf{C}\left(X \oplus Y,_{-}\right) \cong \mathbf{C}\left(X,,_{-}\right) \times \mathbf{C}\left(Y,,_{-}\right)$and $\mathbf{C}\left(0,{ }_{-}\right) \cong 1$ [141].

We collect all enriched categories with finite biproducts and functors preserving them in a category $\mathbf{B P}(\mathbf{V}$-Cat $)$, that is defined as the full subcategory of $\mathbf{c M o n}(\mathbf{V}$-Cat) consisting of all $\mathbf{V}$-categories $\mathbf{C}$ for which the forgetful func-

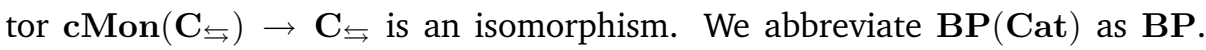

2.3.8 The definition of a biproduct of two objects can easily be generalised to an arbitrary (set-indexed) family of objects. For an index set $I$ and a family $\left(X_{i}\right)$ of objects with $i \in I$, a biproduct is an object $\bigoplus_{i \in I} X_{i}$ that is simultaneously a product with projections $\pi_{j}: \bigoplus_{i \in I} X_{i} \rightarrow X_{j}$, and a coproduct with coprojections $\kappa_{j}: X_{j} \rightarrow \bigoplus_{i \in I} X_{i}$, such that equations (2.4) and (2.5) hold.

Notice that for $I=\emptyset$ this reduces to a zero object; moreover, having finite biproducts reduces to the special case $I=\{1,2\}$.

2.3.9 Example The category Rel has arbitrary biproducts, provided by disjoint union and the empty set. Given an $I$-indexed family of sets $X_{i}$, the biproduct $\bigoplus_{i \in I} X_{i}$ is the disjoint union $\left\{\varphi: I \rightarrow \bigcup_{i \in I} X_{i} \mid \varphi(i) \in X_{i}\right\}$, with projections $\pi_{j}=\left\{(\varphi, \varphi(j)) \mid \varphi \in \bigoplus_{i \in I} X_{i}\right\} \subseteq \bigoplus_{i \in I} X_{i} \times X_{j}$, and coprojections $\kappa_{j}=$ $\left\{(\varphi(j), \varphi) \mid \varphi \in \bigoplus_{i \in I} X_{i}\right\} \subseteq X_{j} \times \bigoplus_{i \in I} X_{i}$.

2.3.10 Example The category cMon has arbitrary products. Given an $I$-indexed family of commutative monoids $X_{i}$, its product is just the Cartesian product $\prod_{i \in I} X_{i}$ of the underlying sets with pointwise operations. This category also has arbitrary coproducts: the coproduct of the above family is the direct sum $\left\{\varphi: I \rightarrow \bigcup_{i \in I} X_{i} \mid \varphi(i) \in X_{i}, \operatorname{supp}(\varphi)\right.$ finite $\}$. The coprojections $\kappa_{j}: X_{j} \rightarrow$ $\bigoplus_{i \in I} X_{i}$ are determined by $\operatorname{supp}\left(\kappa_{j}(x)\right)=\{j\}$ and $\kappa_{j}(x)=x$.

These coincide for finite families, and indeed cMon has finite biproducts. The zero-object is the trivial monoid $\{0\}$.

2.3.11 Example The category Vect $_{K}$ has infinite products and coproducts, too, but only has finite biproducts, in much the same way as the previous example. The biproduct $X_{1} \oplus X_{2}$ is the set $X_{1} \times X_{2}$ with pointwise operations. Projections are given by $\pi_{i}\left(x_{1}, x_{2}\right)=x_{i}$, the coprojections are $\kappa_{1}(x)=(x, 0)$ and $\kappa_{2}(x)=$ $(0, x)$. The zero-object is the 0 -dimensional vector space $\{0\}$.

2.3.12 Example The categories preHilb ${ }_{K}$ and Hilb $_{K}$ inherit finite biproducts from $\operatorname{Vect}_{K}$. The inner product on $X_{1} \oplus X_{2}$ is given by

$$
\left\langle\left(x_{1}, x_{2}\right) \mid\left(y_{1}, y_{2}\right)\right\rangle_{X_{1} \oplus X_{2}}=\left\langle x_{1} \mid y_{1}\right\rangle_{X_{1}}+\left\langle x_{2} \mid y_{2}\right\rangle_{X_{2}} .
$$


This prescription shows that infinite biproducts might be problematic. Indeed, they cannot exist in $\mathbf{H i l b}_{K}$, as the following lemma shows.

2.3.13 Lemma The category Hilb does not have infinite coproducts.

Proof Consider the following counterexample. Define an $\mathbb{N}$-indexed family $X_{n}=\mathbb{C}$ of objects of Hilb. Suppose the family $\left(X_{n}\right)$ had a coproduct $X$ with coprojections $\kappa_{n}: X_{n} \rightarrow X$. Define $f_{n}: X_{n} \rightarrow \mathbb{C}$ by $f_{n}(z)=n \cdot\left\|\kappa_{n}\right\| \cdot z$. These are bounded maps, since $\left\|f_{n}\right\|=n \cdot\left\|\kappa_{n}\right\|$. Then for all $n \in \mathbb{N}$ the norm of the cotuple $f: X \rightarrow \mathbb{C}$ of $\left(f_{n}\right)$ must satisfy

$$
n \cdot\left\|\kappa_{n}\right\|=\left\|f_{n}\right\|=\left\|f \circ \kappa_{n}\right\| \leq\|f\| \cdot\left\|\kappa_{n}\right\|,
$$

so that $n \leq\|f\|$. This contradicts the boundedness of $f$.

2.3.14 The previous lemma implies that Hilb does not have directed colimits (see 4.2 .13 below), either. For example, any colimit of

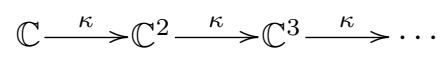

would also be a coproduct of a countable number of copies of $\mathbb{C}$, and hence cannot exist. This invalidates Lemma 5.3 of [112], which observation is due to Peter Johnstone. So Hilb is not a dagger compactly accessible category, as it is called in that article.

2.3.15 In spite of the previous lemma, Hilb does have objects that resemble infinite coproducts, except that they obey the universal property only partially.

Let $I$ be an arbitrary index set, and let $X_{i} \in$ Hilb for all $i \in I$. Put

$$
X=\left\{\left(x_{i}\right)_{i \in I} \in \prod_{i \in I} X_{i} \mid \sum_{i \in I}\left\|x_{i}\right\|^{2}<\infty\right\}
$$

where the product is that of Vect. Here, the sum $\sum_{i \in I} a_{i}$ for an arbitrary (possibly uncountable) index set $I$ is defined as the supremum of $\sum_{i \in J} a_{i}$ where $J$ ranges over the finite subsets of $I$. When $a_{i}$ are all positive, this is welldefined [135]. Equipped with the inner product

$$
\left\langle\left(x_{i}\right)_{i} \mid\left(y_{i}\right)_{i}\right\rangle_{X}=\sum_{i \in I}\left\langle x_{i} \mid y_{i}\right\rangle_{X_{i}}
$$

$X$ becomes a Hilbert space [135].

The object $X$ looks like a biproduct of the objects $X_{i}$. Indeed, there are evident injections $\kappa_{i}: X_{i} \rightarrow X$ defined by $\left(\kappa_{i}(x)\right)_{i}=x$ and $\left(\kappa_{i}(x)\right)_{j}=0$ for $i \neq j$. There are also evident projections $\pi_{i}: X \rightarrow X_{i}$ determined by $\pi_{i}\left(\left(x_{j}\right)_{j}\right)=$ 
$x_{i}$. These satisfy equations (2.4) and (2.5). However, $X$ does not satisfy the universal requirements for a (bi)product, as witnessed by the previous lemma. It is, however, universal in a restricted sense, as follows.

Let us call a cone $g_{i}: Y \rightarrow X_{i}$ bounded when $\sum_{i \in I}\left\|g_{i}\right\|^{2}<\infty$. Then the tuple $g: Y \rightarrow X$ is well-defined by $g(y)=\left(g_{i}(y)\right)_{i \in I}$ and is the unique morphism satisfying $\pi_{i} \circ g=g_{i}$. Likewise, calling a cocone $f_{i}: X_{i} \rightarrow Y$ bounded when $\sum_{i \in I}\left\|f_{i}\right\|^{2}<\infty$, the cotuple $f: X \rightarrow Y$ is well-defined by $f(x)=\sum_{i \in I} f_{i}\left(x_{i}\right)$, and is the unique morphism satisfying $f \circ \kappa_{i}=f_{i}$. Hence we could say that Hilb does not just have finite biproducts, but has bounded biproducts. Note, however, that the cone consisting of projections is not a bounded cone itself. Nor is the cocone of coprojections bounded.

2.3.16 A similar phenomenon already occurs in the category preHilb. For an arbitrary index set $I$ and $X_{i} \in$ preHilb for all $i \in I$, the coproduct of vector spaces $\bigsqcup_{i \in I} X_{i}=\left\{\varphi: I \rightarrow \bigcup_{i \in I} X_{i} \mid \varphi(i) \in X_{i}, \operatorname{supp}(\varphi)\right.$ finite $\}$, equipped with the canonical inner product

$$
\langle\varphi \mid \psi\rangle_{\bigsqcup_{i \in I} X_{i}}=\sum_{i \in I}\langle\varphi(i) \mid \psi(i)\rangle_{X_{i}}
$$

is a well-defined object of preHilb. It inherits the coprojections $\kappa_{j}$ from Vect, as these are adjointable (namely with $\pi_{j}$ as adjoint). However, not all cotuples exist. Given $f_{i}: X_{i} \rightarrow Y$, there is a function $\left[f_{i}\right]_{i \in I}: \coprod_{i \in I} X_{i} \rightarrow Y$ defined by $\varphi \mapsto \sum_{i \in I} f_{i}(\varphi(i))$. If this function were adjointable, its adjoint should be the function $g: Y \rightarrow \coprod_{i \in I} X_{i}$ given by $g(y)(i)=f_{i}^{\dagger}(y)$ in $\coprod_{i \in I} X_{i}$. But the latter function is not well-defined, as its support need not be finite.

The relationship between these phenomena in preHilb and Hilb will be discussed in 3.1.14

2.3.17 In a category with finite biproducts, we can add morphisms $f, g: X \rightrightarrows$ $Y$ by defining $f+g$ as the composite

$$
X \stackrel{\Delta}{\longrightarrow} X \oplus X \stackrel{f \oplus g}{\longrightarrow} Y \oplus Y \stackrel{\nabla}{\longrightarrow} Y .
$$

With the zero morphism $0: X \rightarrow Y$ as unit, this makes every homset into a commutative monoid. The following theorem shows that this works when we start with any $\mathbf{V}$-enriched category with finite products (instead of a Set-category), and moreover that this 'lifting of enrichment' is functorial.

2.3.18 Theorem There is a functor $\left({ }_{(}\right)^{\oplus}: \mathbf{B P}(\mathbf{V}$-Cat $) \longrightarrow(\mathbf{c M o n}(\mathbf{V}))$-Cat. 
Proof Let $(\mathbf{C}, \oplus, 0) \in \mathbf{B P}(\mathbf{V}$-Cat $)$. It comes equipped with maps

$$
\begin{aligned}
& \Delta_{X}: I_{\mathbf{V}} \rightarrow \mathbf{C}(X, X \oplus X), \\
& \nabla_{X}: I_{\mathbf{V}} \rightarrow \mathbf{C}(X \oplus X, X), \\
& u_{X}: I_{\mathbf{V}} \rightarrow \mathbf{C}(0, X), \\
& n_{X}: I_{\mathbf{V}} \rightarrow \mathbf{C}(X, 0) .
\end{aligned}
$$

The objects of $\mathbf{C}^{\oplus}$ are those of $\mathbf{C}$. The carrier of the homobject $\mathbf{C}^{\oplus}(X, Y)$ is $\mathbf{C}(X, Y) \in \mathbf{V}$. Its monoid unit $0_{X Y}: I_{\mathbf{V}} \rightarrow \mathbf{C}(X, Y)$ is given by

$$
0_{X Y}: I_{\mathbf{V}} \stackrel{\cong}{\longrightarrow} I_{\mathbf{V}} \otimes I_{\mathbf{V}} \stackrel{n_{X} \otimes u_{Y}}{\longrightarrow} \mathbf{C}(X, 0) \otimes \mathbf{C}(0, Y) \stackrel{{ }^{\circ} \mathbf{C}}{\longrightarrow} \mathbf{C}(X, Y) .
$$

The monoid multiplication $+: \mathbf{C}(X, Y) \otimes \mathbf{C}(X, Y) \rightarrow \mathbf{C}(X, Y)$ of the homobject $\mathbf{C}(X, Y)$ is given as follows:

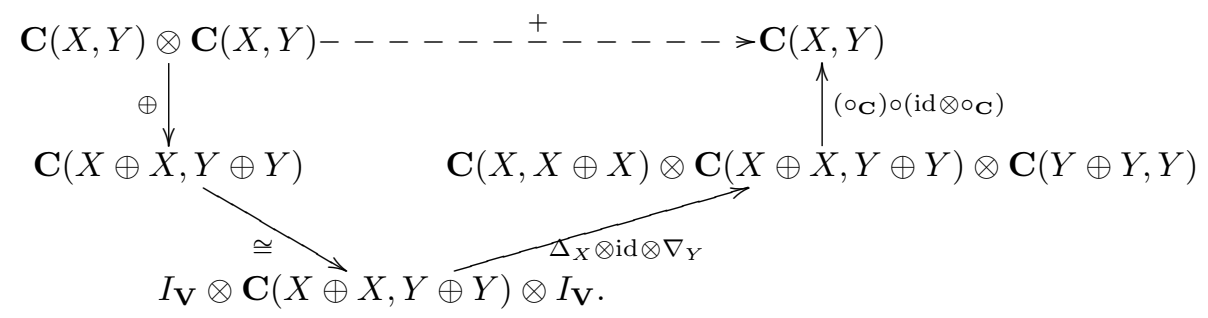

Composition ${ }^{\circ} \mathbf{C}$ is a monoid morphism for this structure because $\oplus$ is a $\mathbf{V}$ functor. We leave it to the reader to show that this data indeed defines a commutative monoid; essentially it is an enriched version of the argument for Setcategories.

Since a morphism in $\mathbf{B P}(\mathbf{V}$-Cat $)$ is a $\mathbf{V}$-functor that strictly preserves the biproduct structure, and because $\Delta, \nabla, n, u$ are natural, this assignment $\mathbf{C} \mapsto$ $\mathbf{C}^{\oplus}$ is functorial.

\subsection{Scalars}

Let us now have a more in-depth look at the scalars introduced in Example 2.2 .8 . We will see that, just like biproducts lift Set-enrichment to cMon-enrichment, tensor products make Set-enrichment into Act(Set)-enrichment. The next section will show that these two liftings of enrichment can be combined. This section shows that, in particular, the scalars have more structure than just that of a monoid. 
2.4.1 A rig is a set $R$ carrying two monoid structures, a commutative one written additively as $(+, 0)$, and the other written multiplicatively as $(\bullet, 1)$, that distribute over each other in the sense that

$$
\begin{aligned}
s \bullet 0 & =0, \\
0 \bullet s & =0, \\
r \bullet(s+t) & =r \bullet s+r \bullet t, \\
(s+t) \bullet r & =s \bullet r+t \bullet r .
\end{aligned}
$$

A rig is called commutative when its multiplication is. Rigs are also known as semirings [94].

Morphisms of rigs are functions that preserve both monoid structures. Thus we have a category $\mathbf{R g}$ of rigs, and a full subcategory $\mathbf{c R g}$ of commutative rigs.

2.4.2 Example There are plenty of examples of rigs. Every (commutative) ring, such as $\mathbb{Z}$, is obviously a (commutative) rig. But $\mathbb{N}$ with its usual addition and multiplication is an example of a rig that is not a ring. In fact, it is an initial object in the category $\mathbf{c R g}$.

Also, every bounded distributive lattice is a commutative rig, with the bottom element as 0 , the top element as 1 , join as + and meet as $\bullet$.

Finally, the set $\mathbb{B}=\{0,1\}$ is called the Boolean rig with operations determined by $1+1=1$.

If a category has finite biproducts as well as tensor products, and each functor $X \otimes \_$has a right adjoint, the tensor products automatically distribute over the biproducts, by

$$
\begin{aligned}
\tau & =\left\langle\text { id } \otimes \pi_{1}, \text { id } \otimes \pi_{2}\right\rangle: X \otimes(Y \oplus Z) \stackrel{\cong}{\longrightarrow}(X \otimes Y) \oplus(X \otimes Z), \\
\tau^{-1} & =\left[\text { id } \otimes \kappa_{1}, \text { id } \otimes \kappa_{2}\right] .
\end{aligned}
$$

This distributivity descends to the scalars, which form another example in of a rig, as in the following lemma.

2.4.3 Proposition The scalars in a (symmetric) monoidal category with finite biproducts form a (commutative) rig.

Proof We already saw in Lemma 2.2.11 that the scalars $\mathbf{C}(I, I)$ in a (symmetric) monoidal category $\mathbf{C}$ form a (commutative) monoid $(\bullet$, id). Also, 2.3.17 showed that if $\mathbf{C}$ also has finite biproducts then its scalars form a commutative monoid under $(+, 0)$. Hence it suffices to verify equations (2.7)-(2.9). Equa- 
tion (2.8) is established by the following commutative diagram.

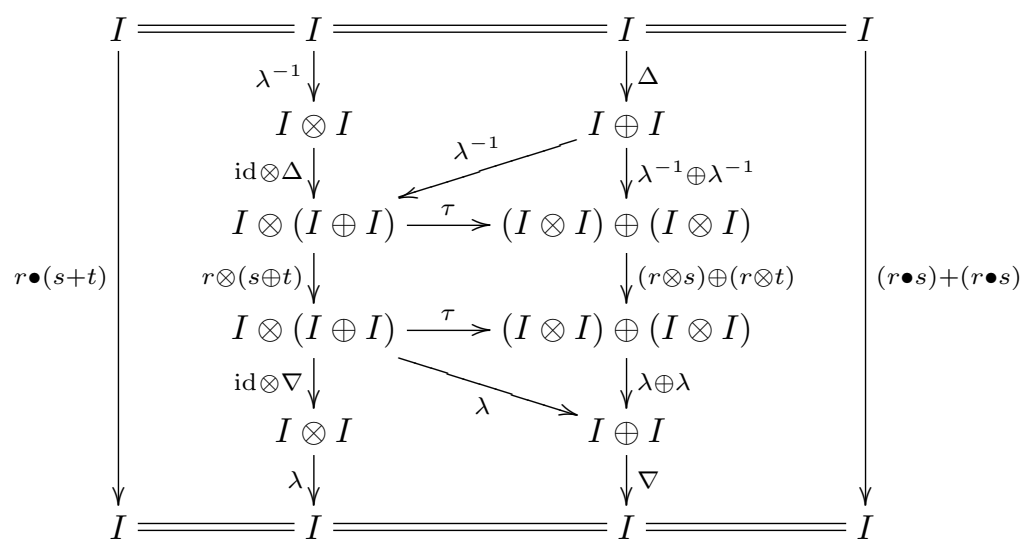

Equation (2.9) is proven analogously. Finally, equation (2.7), and analogously equation (2.6), is shown as follows.

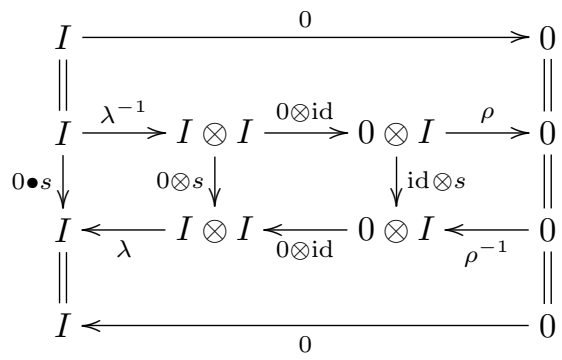

2.4.4 As noted in Example 2.2.18, cMon is a (symmetric) monoidal category. Hence we can consider monoids in it. First of all, an object $R$ of Mon(cMon) is a commutative monoid, which we shall write additively as $(R,+, 0)$. This inner monoid carries a monoid structure $\mathbb{N} \longrightarrow R<-R \otimes R$ in the category cMon. The latter consists of a unit element $1 \in R$ for a binary operation $\bullet: R \times R \rightarrow R$ which is a bimorphism. This means that $\bullet$ is a monoid morphism both in its first variable, i.e. equations (2.7) and (2.8) hold, and in its second variable, i.e. equations (2.6) and (2.9) hold. Hence we can categorically characterise rigs as monoids in the category of commutative monoids: $\mathbf{R g}=\mathbf{M o n}(\mathbf{c M o n})$. Also, we have $\mathbf{c R g}=\mathbf{c M o n}(\mathbf{c M o n})$.

We now set out to prove that scalar multiplication makes a category enriched over actions, in a similar fashion as biproducts make a category enriched over commutative monoids. 
2.4.5 We can use the Kock-Day tensor product for the monad $M \otimes\left(\_\right)$provided by the scalar monoid $M=\mathbf{C}(I, I)$ in any symmetric monoidal category C. This monad is commutative by Lemma 2.2 .14 because the scalar monoid is, by Lemma 2.2.11. The following theorem shows that scalar multiplication in fact provides enrichment, i.e. that composition is a morphism in Act $_{M}$ with respect to the Kock-Day tensor product. Moreover, the enriched category $\mathbf{C}$ and the enriching category $\mathbf{A c t}_{M}$ have 'the same scalars'.

2.4.6 Theorem If a category $\mathrm{C}$ is symmetric monoidal, then it is enriched over $\mathbf{V}=\mathbf{A c t}_{M}$, where $M=\mathbf{C}(I, I)$, in such a way that $I_{\mathbf{V}} \cong \mathbf{C}(I, I)$.

Proof Put the homobject $\mathbf{C}(X, Y) \in \mathbf{V}$ to be the set $\mathbf{C}(X, Y)$ with the action $\mathbf{C}(I, I) \times \mathbf{C}(X, Y) \rightarrow \mathbf{C}(X, Y)$ on it given by scalar multiplication as $(s, f) \mapsto$ $s \bullet f$. The composition morphism $\mathbf{C}(X, Y) \otimes \mathbf{C}(Y, Z) \rightarrow \mathbf{C}(X, Z)$ is now the unique one through which the bimorphism $\mathbf{C}(X, Y) \times \mathbf{C}(Y, Z) \rightarrow \mathbf{C}(X, Z)$, determined by $(f, g) \mapsto g \circ f$, factors; this is a morphism in $\mathbf{V}$. The identity morphism $1 \rightarrow \mathbf{C}(X, X)$ is given by $* \mapsto \mathrm{id}_{X}$; this is also a morphism in $\mathbf{V}$, since 1 carries the trivial action. One easily verifies that these satisfy the requirements of an enriched category. Finally, since the free functor $\mathbf{V} \rightarrow \operatorname{Act}_{M}(\mathbf{V})$ preserves monoidal structure, there is an isomorphism $I_{\mathbf{A c t}_{M}(\mathbf{V})} \cong M \otimes I_{\mathbf{V}} \cong M$ of monoids.

2.4.7 Whereas the previous theorem covers the case of Set-enriched categories, the following theorem gives the general construction. It incorporates functoriality; but for that we first need to get rid of the indexing monoid $M$ in $\operatorname{Act}_{M}(\mathbf{V})$. We denote the Grothendieck completion $\int_{M \in \mathbf{c M o n}(\mathbf{V})} \operatorname{Act}_{M}(\mathbf{V})$ of the indexed category $\mathbf{c M o n}(\mathbf{V})^{\text {op }} \rightarrow$ Cat by $\operatorname{Act}(\mathbf{V})$. Explicitly, $\operatorname{Act}(\mathbf{V})$ has pairs $(M, \alpha)$ with $M \in \mathbf{c M o n}(\mathbf{V})$ and $\alpha \in \operatorname{Act}_{M}(\mathbf{V})$ as objects; morphisms from $(M, \alpha: M \otimes X \rightarrow X)$ to $(N, \beta: N \otimes Y \rightarrow Y)$ in $\mathbf{A c t}(\mathbf{V})$ are pairs of morphisms $f: M \rightarrow N$ and $g: X \rightarrow Y$ in $\mathbf{V}$ satisfying $\beta \circ(f \otimes g)=g \circ \alpha$ [99]. If $\mathbf{V}$ is symmetric monoidal, then so is $\operatorname{Act}(\mathbf{V})$, whence it makes sense to talk of $\operatorname{Act}(\mathbf{V})$-enriched categories.

2.4.8 Theorem If $\mathrm{V}$ is a suitable symmetric monoidal category, there is a functor

$$
\left({ }^{(}\right)^{\otimes}: \operatorname{cMon}(\mathrm{V}-\mathrm{Cat}) \longrightarrow(\operatorname{Act}(\mathrm{V})) \text {-Cat. }
$$

Proof We first describe how $\left({ }_{-}\right)^{\otimes}$ acts on objects. Let $\mathbf{C} \in \mathbf{c M o n}(\mathbf{V}-\mathbf{C a t})$, cf. 2.2.6. The objects of the $\mathbf{V}$-category $\mathbf{C}$ and the $\boldsymbol{A c t}(\mathbf{V})$-category $\mathbf{C}^{\otimes}$ are the same. The homobjects are determined by the action of scalar multiplication, i.e. $\mathbf{C}^{\otimes}(X, Y)$ is the action

$$
\mathbf{C}(I, I) \otimes_{\mathbf{V}} \mathbf{C}(X, Y) \stackrel{\otimes_{\mathbf{C}}}{\longrightarrow} \mathbf{C}\left(I \otimes_{\mathbf{C}} X, I \otimes_{\mathbf{C}} Y\right)=\mathbf{C}(X, Y) .
$$


The identity on $X$ is the morphism

$$
\mathbf{C}(I, I) \stackrel{\cong}{\longrightarrow} \mathbf{C}(I, I) \otimes I_{\mathbf{V}} \stackrel{\mathrm{id} \otimes i_{X}}{\longrightarrow} \mathbf{C}(I, I) \otimes \mathbf{C}(X, X) \stackrel{\otimes_{\mathbf{C}}}{\longrightarrow} \mathbf{C}(X, X)
$$

in V. It is a morphism of actions $I_{\mathbf{A c t}_{\mathbf{C}(I, I)}(\mathbf{V})} \rightarrow \mathbf{C}^{\otimes}(X, X)$ since $I_{\mathbf{A c t}_{M}(\mathbf{V})}$ is the action $\mu: M \otimes M \longrightarrow M$. A lengty but straightforward calculation that uses the structure of the Kock-Day tensor product in $\operatorname{Act}_{M}(\mathbf{V})$, now shows that these data in fact provide an enrichment.

We now turn to the action of $\left(_{-}\right)^{\otimes}$ on morphisms. Let $F$ be a morphism $\mathbf{C} \rightarrow \mathbf{D}$ in $\mathbf{c M o n}\left(\mathbf{V}\right.$-Cat). Define its image $F^{\otimes}$ to work on objects $X \in \mathbf{C}^{\otimes}$ as $F^{\otimes}(X)=F(X)$. It also acts on morphisms as $F$ - since $F$ is a '(strict) monoidal $\mathrm{V}$-functor', it is automatically a (scalar multiplication) action morphism.

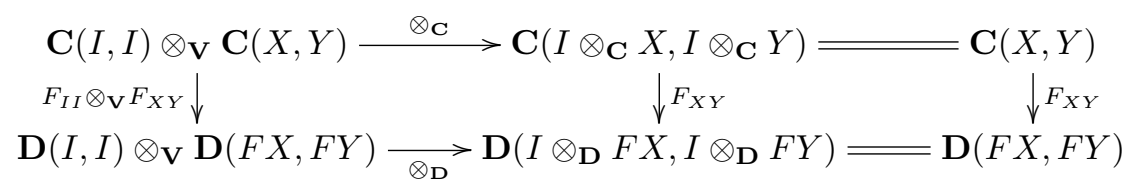

That is, $F_{X Y}^{\otimes}$ is indeed a morphism in $\operatorname{Act}(\mathbf{V})$.

2.4.9 The extension of enrichment in the previous theorem is initial, in the sense that the forgetful functor $\mathbf{C}\left(I_{-}\right): \mathbf{C} \rightarrow \mathbf{V}$ of any symmetric monoidal $\mathbf{V}$-enriched category $\mathbf{C}$ factors through it, as illusstrated in the following commutative diagram of monoidal functors:

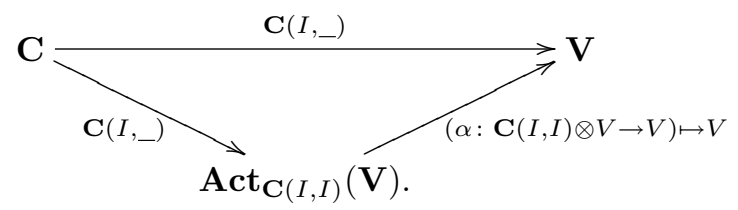

2.4.10 By Lemma 2.2.14, it only makes sense to put the Kock-Day tensor product on $\operatorname{Act}_{\mathbf{C}(I, I)}$ when $\mathbf{C}(I, I)$ is commutative. Hence we can only speak of enrichment in that case. Nonetheless, we can also consider the functor $\mathbf{C}\left(I,_{-}\right)$ when the tensor product in $\mathbf{C}$ is not symmetric. In fact, the following lemma shows that certain homsets have a remnant of the scalar multiplication action, even if a tensor product is not available.

2.4.11 Lemma If $\mathbf{C}$ is a monoidal category, and $s: I \rightarrow I$ and $f: X \rightarrow I$ are morphisms, then $s \bullet f=s \circ f$. Hence even in case $\mathbf{C}$ is not monoidal we have $\mathbf{C}(X, I) \in \mathbf{A c t}_{\mathbf{C}(I, I)}$, and analogously $\mathbf{C}(I, X) \in \mathbf{C}_{\mathbf{C}(I, I)}$ Act. 
PROOF We prove the right-action; the left version is analogous:

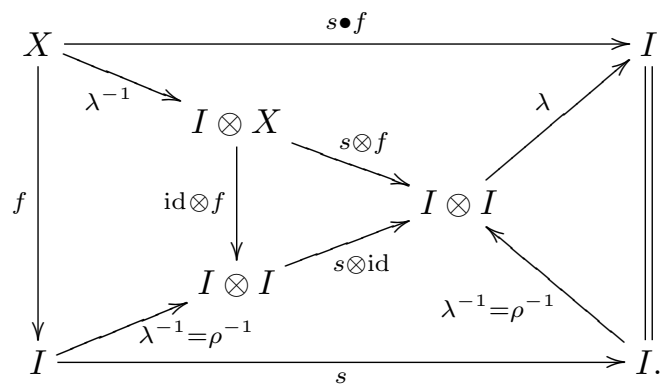

Notice how we use $\lambda_{I}=\rho_{I}$ in much the same way as in Lemma 2.2.11.

\subsection{Modules over rigs}

Part of the structure that descends from the category to the scalars, as in the previous section, applies to all homsets. This section shows that categories with both tensor products and biproducts are enriched in modules over rigs, and uses this to prove preliminary embedding theorems.

2.5.1 A left-module over a rig $R$ is a set $X$ equipped with a commutative addition $(+, 0)$ and a scalar multiplication $\bullet: R \times X \rightarrow X$, satisfying the familiar equations. Right-R-modules are defined analogously, as are left- $R$-right- $S$ modules for rigs $R, S$ (cf. Example 2.1.1). Taking linear functions between modules over a rig $R$ as morphisms, we obtain categories that we denote by ${ }_{R}$ Mod, $\operatorname{Mod}_{R}$ and ${ }_{R} \operatorname{Mod}_{S}$. This overloading of notation is justified because it coincides with the previous meaning in case $R$ is a ring. Modules over rigs are also known as semimodules [94].

2.5.2 Example It is well-known that $\mathbb{Z}$-modules are simply Abelian groups, that is, $\operatorname{Mod}_{\mathbb{Z}} \cong \mathbf{A b}$. A $\mathbb{Q}$-module is precisely a divisible torsion-free Abelian group: $\operatorname{Mod}_{\mathbb{Q}} \cong \operatorname{divtfAb}$. Since an action of $\mathbb{N}$ on a commutative monoid is already completely fixed by its monoid structure, we have $\operatorname{Mod}_{\mathbb{N}} \cong \mathbf{c M o n}$. Looking at the Boolean rig, we can identify an action of $\mathbb{B}=(\{0,1\}$, max, $0, \min , 1)$ on a commutative monoid $L$ as an idempotent commutative monoid, because $l=$ $1 \cdot l=\max (1,1) \cdot l=\max (1 \cdot l, 1 \cdot l)=\max (l, l)$. Hence $\operatorname{Mod}_{\mathbb{B}} \cong$ SLat, the category of bounded semilattices.

2.5.3 Much of the behaviour of $\operatorname{Mod}_{R}$ for $R \in \mathbf{c R g}$ is explained by the fact that it is algebraic. Consider the functor $\widehat{R}$ : Set $\rightarrow$ Set defined on objects by

$$
\widehat{R}(X)=\{\varphi: X \rightarrow R \mid \operatorname{supp}(\varphi) \text { finite }\}
$$


and acting on a function $f: X \rightarrow Y$ as

$$
\widehat{R}(f)(\varphi)(y)=\sum_{x \in f^{-1}(y)} \varphi(x) .
$$

$\widehat{R}$ is a monad in Set. Its unit $\eta: X \rightarrow \widehat{R}(X)$ is given by the Kronecker function

$$
\eta(x)\left(x^{\prime}\right)= \begin{cases}1 & \text { if } x=x^{\prime} \\ 0 & \text { otherwise }\end{cases}
$$

Its multiplication $\mu: \widehat{R}(\widehat{R}(X)) \rightarrow \widehat{R}(X)$ is matrix multiplication

$$
\mu(\Phi)(x)=\sum_{\varphi \in \widehat{R}(X)} \Phi(\varphi) \cdot \varphi(x) .
$$

In fact, $\left.\widehat{\left(\_\right.}\right)$is a functor from $\mathbf{R g}$ to the category of monads on Set. The category of (Eilenberg-Moore) algebras of $\widehat{R}$ is $\operatorname{Mod}_{R}$. Hence, the latter is complete [23, Theorem 3.4.1] and cocomplete [23, Proposition 9.3.4].

In particular, this category has kernels, i.e. equalisers of a morphism and the zero morphism, and cokernels, i.e. coequalisers of a morphism and the zero morphism. Moreover, as the construction of finite biproducts in $\operatorname{Mod}_{R}$ for a ring $R$ does not need subtraction of scalars, it descends to $\operatorname{Mod}_{R}$ for a rig $R$.

2.5.4 However, monos and epis in $\operatorname{Mod}_{R}$ for a rig $R$ might not behave as expected, due to the absence of subtraction of scalars.

A morphism is mono precisely when it is injective. However, not every mono is a kernel. A submodule $X$ of $Y \in \operatorname{Mod}_{R}$ is called subtractive when $y+y^{\prime} \in X$ and $y^{\prime} \in X$ imply $y \in X$ for all $y, y^{\prime} \in Y$. A mono $m: X \rightarrow Y$ in $\operatorname{Mod}_{R}$ is a kernel if and only if $m(X)$ is subtractive. An example of an injective function that is not a kernel is the inclusion $\mathbb{N} \rightarrow \mathbb{Z}$ in $\operatorname{Mod}_{\mathbb{N}}$.

Epimorphisms are harder to characterise. We can, however, recognise regular epimorphisms, i.e. the coequalisers. They are precisely the surjective morphisms. Equivalently, $f: X \rightarrow Y$ is surjective if and only if it is epi and $f(X)$ is subtractive. An example of an epi that is not regular is the inclusion $\mathbb{N} \rightarrow \mathbb{Z}$ in $\operatorname{Mod}_{\mathbb{N}}$. An example of a regular epi that is not a cokernel is $f: \mathcal{P}\{0,1\} \rightarrow\{0,1\}$ determined by $f(\emptyset)=0$ and $f(\{0\})=f(\{1\})=f(\{0,1\})=1$.

2.5.5 Definition An object $G$ is a generator when $f=g: X \rightrightarrows Y$ whenever $f \circ x=g \circ x$ for all $x: G \rightarrow X$. This is precisely what is needed to make $\mathbf{C}\left(G, \_\right)$ faithful. For example, the rig $R$ itself is a generator in $\operatorname{Mod}_{R}$.

An object $P \in \mathbf{C}$ is projective when for every regular epi $X \rightarrow Y$, any mor- 
phism $P \rightarrow Y$ factors through $X$ :

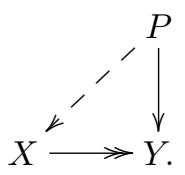

Hence $P$ is projective precisely when $\mathbf{C}\left(P,_{-}\right)$preserves regular epis. The projective objects in $\operatorname{Mod}_{R}$ for a rig $R$ are precisely the projective modules, i.e. the retracts of free ones. In particular, the rig $R$ itself, seen as a (free) module, is a projective object.

An object $X$ is called finitely projective when there are a projective generator $G$, some natural number $n$, and a regular epi $\bigoplus_{i=1}^{n} G \rightarrow X$.

2.5.6 As in 2.4.4, we can characterise modules over rigs categorically. As a rig $R$ is an object in Mon(cMon), we can consider its category of actions ${ }_{R}$ Act(cMon). An object in this category consists of a commutative monoid $(X,+, 0)$ with an action $\bullet: R \otimes X \rightarrow X$. The latter is a morphism in $\mathbf{c M o n}$, and hence corresponds to a bimorphism $\bullet: R \times X \rightarrow X$ in Set. As such it satisfies the action equations

$$
\begin{aligned}
1 \bullet x & =x, \\
(r \bullet s) \bullet x & =r \bullet(s \bullet x),
\end{aligned}
$$

the equations for being a left homomorphism, and those for a right homomorphism:

$$
\begin{aligned}
0 \bullet x & =0, \\
(r \bullet x)+(s \bullet x) & =(r+s) \bullet x, \\
r \bullet 0 & =0, \\
(r \bullet x)+(r \bullet y) & =r \bullet(x+y) .
\end{aligned}
$$

In other words, $(X,+, 0, \bullet)$ is precisely a module over the rig $R$. Also, morphisms in this category are precisely linear functions. Hence ${ }_{R} \operatorname{Mod}={ }_{R} \operatorname{Act}(\mathbf{c M o n})$. We can now define Mod $=\mathbf{A c t}(\mathbf{c M o n})$ as the category of modules over any rig.

Now, Theorems 2.3 .18 and 2.4 .8 can be combined, showing that categories with both biproducts and tensor products are in fact enriched in modules over their scalars. The stock example is the category $\operatorname{Mod}_{R}$ itself, in which the homsets are again $R$-modules by pointwise addition and scalar multiplication. Be- 
cause of equation (2.10), we can speak of strict monoidal categories with finite biproducts as objects of $\operatorname{Mon}(\mathbf{B P})$.

2.5.7 Theorem There is a functor

$$
\left({ }^{-}\right)^{\oplus \otimes}: \operatorname{cMon}(\mathbf{B P}) \longrightarrow \text { Mod-Cat. }
$$

Proof First, notice that the functor $\left(\_\right)^{\oplus}$ of Theorem 2.3.18 is strong monoidal. That is, $(\mathbf{C} \times \mathbf{D})^{\oplus} \cong \mathbf{C}^{\oplus} \times \mathbf{D}^{\oplus}$ for $\mathbf{C}, \mathbf{D} \in \mathbf{c M o n}(\mathbf{B P})$. Moreover, $\mathbf{1}^{\oplus}=\mathbf{1}$ [34]. Hence $\left(\_\right){ }^{\oplus}$ restricts to a functor

$$
\operatorname{cMon}\left(\left({ }_{-}\right)^{\oplus}\right): \operatorname{cMon}(\mathrm{BP}) \longrightarrow \operatorname{cMon}(\mathbf{c M o n}-\mathrm{Cat}) .
$$

The desired functor is then the composition $\left({ }_{-}\right)^{\oplus \otimes}=\left({ }_{(}\right)^{\otimes} \circ \mathbf{c M o n}\left(\left({ }_{-}\right)^{\oplus}\right)$ with the functor from Theorem 2.4.8.

2.5.8 If we combine all functors so far with forgetful ones, we can summarise Theorems 2.3.18, 2.4.8 and 2.5.7 by the following diagram:

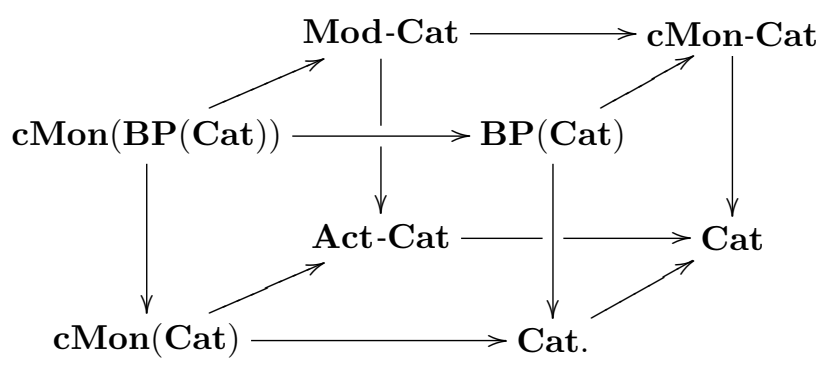

The next corollary is the basis for several embedding theorems.

2.5.9 Corollary If $\mathbf{C}$ is a symmetric monoidal category with biproducts, then there is a functor $\mathbf{C}\left(I,{ }_{-}\right): \mathbf{C} \rightarrow \operatorname{Mod}_{R}$, where $R=\mathbf{C}(I, I)$. It is faithful if and only if $I$ is a generator.

Following Lemma 2.4.11, we can extend the previous corollary to the situation where $\mathbf{C}$ does not have tensor products.

2.5.10 Theorem If $\mathbf{C}$ has biproducts, then there is a functor $\mathbf{C}\left(I,{ }_{-}\right): \mathbf{C} \rightarrow{ }_{R} \mathbf{M o d}$ for any object $I \in \mathbf{C}$, where $R=\mathbf{C}(I, I)$. This functor:

(a) is faithful if and only if I is a generator;

(b) is full when I is a projective generator and every object of $\mathbf{C}$ is finitely projective; 
(c) preserves finite biproducts;

(d) preserves coequalisers and hence all finite colimits if and only if I is projective;

(e) is monoidal when $\mathbf{C}$ is monoidal and $I$ is its monoidal unit.

Proof We already know that $\mathbf{C}\left(I,_{-}\right)$can be regarded as a functor to $\mathbf{c M o n}$ as well as to ${ }_{R} \mathbf{A c t}(\mathbf{S e t})$. Hence to prove that it is a functor to ${ }_{R}$ Mod, it suffices to show that $\mathbf{C}(I, X)$ has the distribution properties (2.14)-(2.17). Equations (2.14) and (2.16) are immediate: $0 \bullet x=x \circ 0=0$, and equations (2.15) and (2.17) follow from properties of the biproduct:

$$
\begin{aligned}
(r \bullet x)+(s \bullet x) & =(x \circ r)+(x \circ s) \\
& =\nabla \circ((x \circ r) \oplus(x \circ s)) \circ \Delta \\
& =\nabla \circ(x \oplus x) \circ(r \oplus s) \circ \Delta \\
& =x \circ \nabla \circ(r \oplus s) \circ \Delta \\
& =x \circ(r+s) \\
& =(r+s) \bullet x .
\end{aligned}
$$

Right-distributivity is analogous.

In proving part (b), we temporarily write the action of $\mathbf{C}\left(I,{ }_{-}\right)$on morphisms as $T: \mathbf{C}(X, Y) \rightarrow{ }_{R} \operatorname{Mod}(\mathbf{C}(I, X), \mathbf{C}(I, Y))$. Let $\Phi: \mathbf{C}(I, X) \rightarrow \mathbf{C}(I, Y)$ in ${ }_{R}$ Mod. We must find $\varphi: X \rightarrow Y$ in $\mathbf{C}$ such that $\Phi(x)=\varphi \circ x$ for all $x: I \rightarrow X$ in $\mathbf{C}$.

Suppose first that $I=X$. Then $\Phi(x)=\Phi\left(\mathrm{id}_{I} \circ x\right)=\Phi\left(\mathrm{id}_{I}\right) \circ x$ since $\Phi$ is a morphism of left- $R$-modules. So $\varphi=\Phi\left(\mathrm{id}_{I}\right)$ satisfies $\Phi(x)=\varphi \circ x$ for all $x: I \rightarrow X$.

In general, since $X$ is finitely projective, there are an $n \in \mathbb{N}$ and a regular epi $p: \bigoplus_{i=1}^{n} I \rightarrow X$. Denote by $\Phi_{i}$ the morphism

$$
\Phi_{i}: \mathbf{C}(I, I) \stackrel{T\left(\kappa_{i}\right)}{\longrightarrow} \mathbf{C}\left(I, \bigoplus_{i=1}^{n} I\right) \stackrel{T(p)}{\longrightarrow} \mathbf{C}(I, X) \stackrel{\Phi}{\longrightarrow} \mathbf{C}(I, Y) \quad \text { in }{ }_{R} \text { Mod. }
$$

So, by the previous case $(I=X)$, for each $i \in I$ there is $\varphi_{i} \in \mathbf{C}(I, Y)$ such that $\Phi_{i}(x)=\varphi_{i} \circ x$ for all $x \in S$. Define $\bar{\varphi}=\left[\varphi_{i}\right]_{i=1}^{n}: \bigoplus_{i=1}^{n} I \rightarrow Y$, and write $\bar{\Phi}=\Phi \circ T(p): \mathbf{C}\left(I, \bigoplus_{i=1}^{n} I\right) \rightarrow \mathbf{C}(I, Y)$. Then, for $x \in \mathbf{C}\left(I, \bigoplus_{i=1}^{n} I\right)$ :

$$
\begin{aligned}
\bar{\Phi}(x) & =\Phi(p \circ x)=\Phi\left(p \circ\left(\sum_{i \in I} \kappa_{i} \circ \pi_{i}\right) \circ x\right)=\sum_{i=1}^{n} \Phi\left(p \circ \kappa_{i} \circ \pi_{i} \circ x\right) \\
& =\sum_{i=1}^{n} \Phi_{i}\left(\pi_{i} \circ x\right)=\sum_{i=1}^{n} \varphi_{i} \circ \pi_{i} \circ x=\bar{\varphi} \circ x .
\end{aligned}
$$


Since $p$ is a regular epi, it is a coequaliser, say

$$
M \stackrel{m^{\prime}}{\underset{m}{\longrightarrow}} \bigoplus_{i=1}^{n} I \stackrel{p}{\longrightarrow} X \text {. }
$$

Now,

$$
\bar{\varphi} \circ m=\bar{\Phi}(m)=\Phi(p \circ m)=\Phi\left(p \circ m^{\prime}\right)=\bar{\Phi}\left(m^{\prime}\right)=\bar{\varphi} \circ m^{\prime},
$$

so there is a (unique) $\varphi: X \rightarrow Y$ such that $\bar{\varphi}=\varphi \circ p$. Let $x: I \rightarrow X$. Because $I$ is projective, there is an $x^{\prime}: I \rightarrow \bigoplus_{i=1}^{n} I$ such that $x=p \circ x^{\prime}$. Finally

$$
\Phi(x)=\Phi\left(p \circ x^{\prime}\right)=\bar{\Phi}\left(x^{\prime}\right)=\bar{\varphi} \circ x^{\prime}=\varphi \circ p \circ x^{\prime}=\varphi \circ x .
$$

Thus $\mathbf{C}\left(I,{ }_{-}\right)$is full.

As for (c): since biproducts are in particular products, we have $\mathbf{C}(I, X \oplus Y) \cong$ $\mathbf{C}(I, X) \oplus \mathbf{C}(I, Y)$ because of the universal property of a product. Then (d) follows immediately.

Finally, to prove (e), suppose that $\mathbf{C}$ is monoidal. By definition we have $R=\mathbf{C}(I, I)$. We still need to exhibit a natural transformation with components $\mathbf{C}(I, X) \otimes \mathbf{C}(I, Y) \rightarrow \mathbf{C}(I, X \otimes Y)$ in ${ }_{R}$ Mod. That is, we need a bimorphism $\mathbf{C}(I, X) \times \mathbf{C}(I, Y) \rightarrow \mathbf{C}(I, X \otimes Y)$. But that is easy: map $x: I \rightarrow X$ and $y: I \rightarrow Y$ to the composite $I \stackrel{\cong}{\longrightarrow} I \otimes I \stackrel{x \otimes y}{\longrightarrow} X \otimes Y$. One easily verifies that this is natural, bilinear, which satisfies the required coherence properties.

We conclude this section with some further context, summarising well-known results and placing the developments leading to the previous theorem in the perspective of established literature.

2.5.11 For certain well-behaved categories $\mathbf{C}$ with finite biproducts, the previous theorem can be strengthened by prefacing it with a functor that embeds $\mathrm{C}$ into a category with finite biproducts and a projective generator. This is accomplished by the process of Lubkin completion due to Saul Lubkin and was further developed by Michael Barr [19, 34, 159]. It works for regular categories, i.e. categories with coequalisers of kernel pairs in which regular epimorphisms are stable under pullbacks. As a corollary to 2.5.3 — and as might be expected from 2.5.4 - the category $\operatorname{Mod}_{R}$ for $R \in \mathbf{R g}$ is regular. We now summare this construction. We do not pursue full detail, as the next chapter considers a novel embedding theorem of categories for which the Lubkin completion cannot be performed in principle.

2.5.12 Theorem (Lubkin-Barr) If $\mathbf{C}$ is a small regular category, there are a small cMon-enriched regular category $\mathbf{D}$ and a full and faithful functor $\mathbf{C} \rightarrow[\mathbf{D}, \mathbf{c M o n}]$ 
that preserves finite limits and coequalisers. ${ }^{1}$

If $\mathbf{C}$ furthermore has finite biproducts, this functor automatically preserves all finite colimits as well.

Proof By Example 2.2.18, the category cMon satisfies the conditions set out in [19, III.5.12]. Hence the statement follows from [19, Theorem III.1.3].

The next few lemmas show explicitly that the cMon-category [D, cMon] of the previous theorem has a projective generator, namely the functor

$$
U=\coprod_{X \in \mathbf{D}} \mathbf{D}\left(X, \_\right): \mathbf{D} \rightarrow \mathbf{c M o n} .
$$

\subsubsection{Lemma $U$ is a projective object in [D, cMon].}

Proof Since a coproduct of projectives is again projective [33, Proposition 4.6.7], it suffices to show that for $X \in \mathbf{D}$, the representable functor $\mathbf{D}\left(X,{ }_{-}\right)$is projective in $[\mathbf{D}, \mathbf{c M o n}]$. For $F, G \in[\mathbf{D}, \mathbf{c M o n}]$, let $\alpha: \mathbf{D}\left(X,{ }_{-}\right) \rightarrow F$, and let $\beta: G \rightarrow$ $F$ be a regular epi. Since $\alpha$ is just a natural transformation, it corresponds to an $f \in F(X)$ via the (enriched) Yoneda lemma. Likewise, $\beta$ is a natural transformation, the components $\beta_{Y}: G(Y) \rightarrow F(Y)$ of which are regular epis in $\mathbf{c M o n}$ since colimits in functor categories are computed pointwise. That is, every $\beta_{Y}$ is a surjective monoid homomorphism. Pick $g \in \beta_{X}^{-1}(f) \subseteq G(X)$. Again via the Yoneda lemma, $g$ corresponds to a natural transformation $\gamma: \mathbf{C}\left(X,_{-}\right) \rightarrow G$, and $\beta \circ \gamma=\alpha$. Hence $\mathbf{D}\left(X,{ }_{-}\right)$is projective.

2.5.14 Lemma Let $\mathbf{D}$ be a regular category and $P$ a projective object in it. If for distinct subobjects $r: R \longmapsto Y$ and $s: S \longmapsto Y$, there is an $y: P \rightarrow S$ not factoring through $r$, (or an $y: P \rightarrow R$ not factoring through $s$ ), then $P$ is a generator.

PROOF In any regular category, any morphism $f: X \rightarrow Y$ can be written as a composition $X \longrightarrow \operatorname{Im}(f) \longrightarrow Y$ of a regular epi and a mono (see Example 3.4.13). Now, given $f \neq g: X \rightrightarrows Y$, their images $\operatorname{Im}(f) \longmapsto Y$ and $\operatorname{Im}(g) \longmapsto$ $Y$ are distinct. So there is an $y: P \rightarrow \operatorname{Im}(f)$ not factoring through $\operatorname{Im}(g)$ :

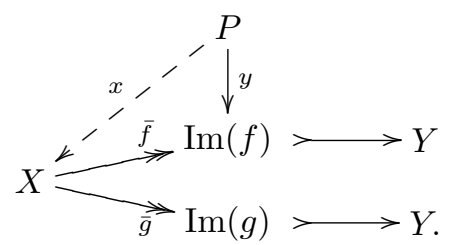

\footnotetext{
${ }^{1}$ In the context of Theorem $\sqrt{2.5 .10}$ (e) and Definition 3.6.1 in the next chapter, it is noteworthy that the objects of $\mathbf{D}$ bijectively correspond to subobjects of the terminal object whenever $\mathbf{C}$ has equalisers. [19, Theorem III.1.6]
} 
So there is an $x: P \rightarrow X$ such that $\operatorname{Im}(f) \circ y=\operatorname{Im}(f) \circ \bar{f} \circ x=f \circ x$. If $f \circ x=g \circ x$, then $\operatorname{Im}(f) \circ y=\operatorname{Im}(g) \circ \bar{g} \circ x$, so $y$ would factor through $\operatorname{Im}(g)$. Hence $f \circ x \neq g \circ x$, and thus $P$ is a generator.

\subsubsection{Lemma $U$ is a generator for $[\mathrm{D}, \mathrm{cMon}]$.}

PROOF By the previous lemma, it suffices to prove that for distinct subobjects $\alpha: S \Rightarrow F$ and $\beta: T \Rightarrow F$, there is a $\gamma: U \Rightarrow S$ not factoring through $T$. Since $\alpha \neq \beta$, we can assume there is a $Z \in \mathbf{D}$ with $\alpha_{Z}: S(Z) \longmapsto F(Z)$ not factoring through $\beta_{Z}: T(Z) \longmapsto F(Z)$. Pick an element $w \in \alpha_{Z}(S(Z))$ such that $w \notin$ $\beta_{Z}(T(Z))$, say $w=\alpha_{Z}(z)$ for $z \in S(Z)$. Define $c \in \prod_{X \in \mathbf{C}} S(X)$ by $\pi_{Z}(c)=z$ and $\operatorname{supp}(c)=\{X\}$. By the Yoneda lemma

$$
\left[\coprod_{X \in \mathbf{C}} \mathbf{C}\left(X,{ }_{-}\right), S\right] \cong \prod_{X \in \mathbf{C}}\left[\mathbf{C}\left(X,{ }_{-}\right), S\right] \cong \prod_{X \in \mathbf{C}} S(X) .
$$

Denote the natural transformation corresponding to $c$ by $\gamma: \amalg_{X \in \mathbf{C}} \mathbf{C}\left(X,{ }_{-}\right) \Rightarrow$ $S$, and the element corresponding to $\prod_{X \in \mathbf{C}}\left[\mathbf{C}\left(X, \_\right), S\right]$ by $\gamma^{\prime}$. If $\gamma$ were to factor through $T$, then $\gamma_{Z}^{\prime}: \mathbf{C}\left(Z,{ }_{-}\right) \Rightarrow S$ would factor through $T$. In particular, then $\pi_{Z}\left(\gamma_{Z}^{\prime}\right): \mathbf{C}(Z, Z) \rightarrow S(Z)$ would factor through $T(Z)$, whence $\pi_{Z}(c)=z \in S(Z)$ would factor through $T(Z)$ via $\beta_{Z}$. But this is a contradiction, and hence $\gamma$ does not factor through $T$.

Since the construction of the functor in Theorem 2.5.12 is such that its image consists of finitely projective objects [19, Proposition III.5.10], we can conclude with the following theorem.

2.5.16 Theorem Any small regular category with finite biproducts has a full embedding into the category of modules over a rig that preserves all finite colimits.

\subsection{Compact objects}

This section studies objects that can be 'reversed' in a particular sense. In quantum theory, the relationship between such a 'reversible' object and its dual accounts for the information flow between entangled particles-this perspective will be exemplified in Section 3.3. In standard cases, compact objects turn out to correspond to finite-dimensional modules under the embedding theorem of the previous section.

2.6.1 Before we formally define the notion of a compact object, let us discuss the terminology "compact", for an object, or "compact closed", for a category in which every object is compact. As we will see in Example 3.2.14, the category 
[ $G$, fdHilb] of finite-dimensional unitary representations of a given group $G$ is a compact closed category. This example is where the compactness terminology seems to have originated: the group $G$ can be reconstructed from [ $G$, fdHilb] when it is compact [132, 149, 207]. Hence the name "compact" transferred from the group to categories resembling $[G$, fdHilb].

Alternatively, one could observe that being a Hausdorff space, the unit ball of a Hilbert space (in its norm topology) is compact if and only if it is finitedimensional. Finally, a Hilbert space is locally compact if and only if it is finitedimensional [104, Problem 10].

Compact closed categories were first introduced in 1972 as a class of examples in the context of the coherence problem [140]. They were subsequently studied first from the perspective of categorical algebra [65, 142], and later in relation to linear logic [195]. Interest has rejuvenated since the discovery of the aspect that we use in this thesis: compact closed categories model (aspects of) quantum computation [2, 197]. However, none of these references explain the origin of the name "compact".

Compact closed categories and closely related variants that are not necessarily symmetric are known under an abundance of different names in the literature: rigid, pivotal, autonomous, sovereign, spherical, ribbon, tortile, balanced, category with conjugates [200].

2.6.2 Definition An object $X$ of a symmetric monoidal category is said to be compact when it carries a compact structure, i.e. there are an object $Y$ and morphisms $\eta: I \rightarrow Y \otimes X$ and $\varepsilon: X \otimes Y \rightarrow I$ such that the following diagrams commute:
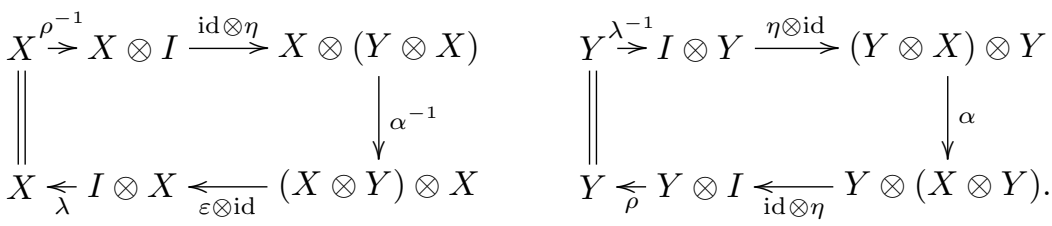

A compact closed category is a symmetric monoidal category whose objects are compact.

2.6.3 For a given compact object $X$, the object $Y$ of the previous definition is called a dual object for $X$. Such dual objects are unique up to isomorphism [188, 2.5.4]. A chosen dual object for $X$ is usually denoted by $X^{*}$. Notice that $I$ is a compact object in any symmetric monoidal category, with $I^{*}=I$. Also, if $X$ is compact, then so is $X^{*}$. Moreover, any compact object $X$ is isomorphic to its double dual $X^{* *}[188,2.5 .4 .3]$. 
2.6.4 A bicategory is a category that is weakly enriched over Cat, in the sense that composition is only associative up to isomorphism-see [26], [157] or [33, Section 7.7]. For example, the category Mod may be regarded as a bicategory, whose objects are commutative rings.

Any monoidal category may be regarded as a one-object bicategory, whose single homobject is the given category, and whose composition is provided by the given monoidal structure. As in any 2-category, it makes sense to consider adjunctions in a bicategory. Two compact objects in a symmetric monoidal category are dual to each other precisely when they are both left and right adjoint to each other as morphisms in the induced one-object bicategory. Under this identification, $\eta$ and $\varepsilon$ are the unit and counit of the adjunction [65, 158].

2.6.5 Example In a posetal symmetric monoidal category, diagrams (2.18) say that an object $X$ is compact precisely when there is an object $X^{*}$ such that $X^{*} \otimes X=I=X \otimes X^{*}$. Any ordered commutative monoid is such a category, where the order induces the morphisms, and the monoid multiplication and unit provide symmetric monoidal structure. Hence the compact objects in an ordered commutative monoid, seen as a posetal category, are precisely its invertible elements. Thus any ordered Abelian group induces a compact closed category; an Abelian group is partially ordered if and only if it is torsion-free [171, Theorem 1.1.3]. This example has been studied more generally under the name of "Lambek pregroups" [189].

2.6.6 Example In the category Vect, any finite-dimensional vector space $X$ is a compact object, with compact structure as follows. Let $X^{*}$ be the dual vector space $\{f: X \rightarrow \mathbb{C} \mid f$ linear $\}$. If $\left(e_{i}\right)$ is a basis for $X$, then the functionals $\theta^{i}$ determined by $\theta^{i}\left(e_{i}\right)=1$ and $\theta^{i}\left(e_{j}\right)=0$ when $i \neq j$ form a basis for $X^{*}$. Define $\eta_{X}$ and $\varepsilon_{X}$ by linear extension of

$$
\begin{aligned}
\eta_{X}(1) & =\sum_{i=1}^{\operatorname{dim}(X)} \theta^{i} \otimes e_{i}, \\
\varepsilon_{X}\left(e_{i} \otimes \theta^{j}\right) & =\theta^{j}\left(e_{i}\right) .
\end{aligned}
$$

Diagrams (2.18) are readily seen to commute.

An infinite-dimensional vector space cannot be isomorphic to its double dual because of a well-known cardinality argument [127, Theorem IX.2], which we sketch briefly. Let $X$ be an infinite-dimensional vector space, and choose a basis $B$ for it. Then $X \cong \bigsqcup_{B} \mathbb{C}$, and so $X^{*} \cong \prod_{B} \mathbb{C}$ [8, Proposition 20.2]. So $\operatorname{dim}(X) \supsetneqq \operatorname{dim}\left(X^{*}\right) \supsetneqq \operatorname{dim}\left(X^{* *}\right)$, whence $X \not X^{* *}$ and $X$ is not a compact object in Vect. For completeness' sake, let us recall here that even for a finitedimensional vector space $X$, the isomorphism $X \cong X^{*}$ is not natural, although 
$X \cong X^{* *}$ is [163, Section VII.4]. Thus the full subcategory fdVect of Vect consisting of finite-dimensional vector spaces is the largest compact closed subcategory of Vect.

The following lemma generalises this example to the category of modules over a commutative rig $R$ : the compact objects in that category are precisely the finitely projective ones. Recall from Example 2.1.1 that a module over $R$ is called free if it is of the form $R^{X}$ for some $X \in$ Set, with pointwise operations; the cardinality of $X$ is its dimension. A module is called projective if it is a retract of a free one, and finitely projective if it is a retract of a finite-dimensional free one.

2.6.7 Lemma For a commutative rig $R$, an object $X \in \operatorname{Mod}_{R}$ is compact if and only if it is finitely projective.

Proof [103, Lemma 1.3] The category $\operatorname{Mod}_{R}$ is enriched over itself. So if $X$ is compact, there is an isomorphism $a: \operatorname{Mod}_{R}(X, X) \rightarrow X \otimes \operatorname{Mod}_{R}(X, R)$ in $\operatorname{Mod}_{R}$. By the structure of the tensor product in $\operatorname{Mod}_{R}$, we can write $a\left(\operatorname{id}_{X}\right)=$ $\sum_{i=1}^{n} x_{i} \otimes \varphi^{i}$ for some $x_{i} \in X$ and $\varphi^{i}: X \rightarrow R$ and $i=1, \ldots, n$. Define $f: X \rightarrow$ $R^{n}$ by $f(x)=\left(\varphi^{1}(x), \ldots, \varphi^{n}(x)\right)$, and $g: R^{n} \rightarrow X$ by $g\left(r_{1}, \ldots, r_{n}\right)=\sum_{i=1}^{n} r_{i} \cdot x_{i}$. Diagrams (2.18) then yield $\sum_{i=1}^{n} \varphi^{i}(x) \cdot x_{i}=x$, so that $g \circ f=\operatorname{id}_{X}$. Hence $X$ is a retract of the finite-dimensional free module $R^{n}$.

We now recall a few basic properties of compact objects that will be useful later. The following proposition shows that compact closed categories are indeed closed.

2.6.8 Proposition [158] Let $\mathbf{C}$ be a symmetric monoidal category.

(a) If $X \in \mathbf{C}$ is compact, then $\mathbf{C}(X, Y) \cong \mathbf{C}\left(I, X^{*} \otimes Y\right)$ for all $Y \in \mathbf{C}$.

(b) If $Y \in \mathbf{C}$ is compact, then $\mathbf{C}(X, Y) \cong \mathbf{C}\left(X \otimes Y^{*}, I\right)$ for all $X \in \mathbf{C}$.

(c) An object $X \in \mathbf{C}$ is compact if and only if there is an $Y \in \mathbf{C}$ such that $\mathbf{C}(X \otimes Y, I) \cong \mathbf{C}(X, X) \cong \mathbf{C}(I, Y \otimes X)$.

(d) An object $X \in \mathbf{C}$ is compact iff there is a $Y \in \mathbf{C}$ such that $X \otimes\left({ }_{-}\right)$is left adjoint to $Y \otimes\left({ }_{(}\right)$. In that case, $X \otimes\left({ }_{-}\right)$is also right adjoint to $Y \otimes\left(\_\right)$.

2.6.9 For future reference, let us explicate the bijections in (a) and (b) of the previous proposition. In (a), a morphism $f: X \rightarrow Y$ corresponds to its name $\ulcorner f\urcorner=(f \otimes$ id $) \circ \eta: I \rightarrow X^{*} \otimes Y$. It satisfies the following absorption rules [77, Lemma 2.18]:

$$
\text { (id } \otimes g) \circ\ulcorner f\urcorner=\ulcorner g \circ f\urcorner=\left(f^{*} \otimes \text { id }\right) \circ\ulcorner g\urcorner \text {. }
$$


Likewise, in (b), $f$ corresponds to its coname $\llcorner f\lrcorner=\varepsilon \circ(f \otimes$ id $): X \otimes Y^{*} \rightarrow I$.

A prominent property of a compact closed category is that a choice of dual objects $X^{*}$ extends functorially, as in the following proposition.

2.6.10 Proposition [142] For a morphism $f: X \rightarrow Y$ in a compact closed category $\mathbf{C}$, define $f^{*}: Y^{*} \rightarrow X^{*}$ as the composite

$$
Y^{*} \cong Y^{*} \otimes I \stackrel{\mathrm{id} \otimes \eta_{X}}{\longrightarrow} Y^{*} \otimes\left(X \otimes X^{*}\right) \stackrel{\mathrm{id} \otimes f \otimes \mathrm{id}}{\longrightarrow}\left(Y^{*} \otimes Y\right) \otimes X^{*} \stackrel{\varepsilon_{Y} \otimes \mathrm{id}}{\longrightarrow} I \otimes X^{*} \cong X^{*} .
$$

This defines a "choice-of-duals" functor $\left({ }_{(}\right)^{*}: \mathbf{C}^{\mathrm{op}} \rightarrow \mathbf{C}$. In fact, $\left({ }_{(}\right)^{*}$ is an equivalence since $\left(\_\right)^{* *} \cong \mathrm{Id}$.

We end this chapter by making precise the intuition that compact objects 'behave finite-dimensionally' in standard cases, by considering their image under the embedding of Theorem 2.5.10.

2.6.11 Proposition Let $\mathbf{C}$ be a symmetric monoidal category with finite biproducts, and denote its scalars by $R=\mathbf{C}(I, I)$. Suppose that the monoidal functor $\mathbf{C}\left(I,{ }_{-}\right): \mathbf{C} \rightarrow \operatorname{Mod}_{R}$ of Theorem 2.5 .10 is strong monoidal. If $X$ is a compact object in $\mathbf{C}$, then $\mathbf{C}(I, X)$ is finitely projective.

PROOF Strong monoidal functors preserve compact objects [158, Proposition 3], and Lemma 2.6.7 shows that the compact objects in $\operatorname{Mod}_{R}$ are precisely the finitely projective $R$-modules.

2.6.12 The fact that the functor $\mathbf{C}\left(I,_{-}\right)$is strong monoidal means that the canonical coherent morphisms

$$
\mathbf{C}(I, X) \otimes \mathbf{C}(I, Y) \cong \mathbf{C}(I, X \otimes Y)
$$

are isomorphisms. Here, the tensor product on the left-hand side is that of $R$ modules, i.e. the Kock-Day tensor product of Example 2.2.18. Hence the requirement in the previous proposition means that the tensor product of $\mathbf{C}$ "behaves bilinearly". This is quite a natural restriction. 



\section{Chapter 3}

\section{Dagger categories}

This chapter studies categories in which one can reverse morphisms. That is, categories with a self-duality that is made explicit by a so-called dagger. Possible extra structure, such as the tensor products and biproducts studied in the previous chapter, is then required to be compatible with this dagger. The presence of a dagger turns out to have profound consequences. For example, we will prove that the scalars in a Hilbert category - a special kind of dagger category with biproducts and tensor products-form a subfield of the complex numbers, and that the whole category embeds into that of Hilbert spaces. The material in this chapter is based on [114] and [116].

\subsection{Examples}

After formally defining the object of study, this section considers several examples: first concrete ones, and then more general ones.

3.1.1 Definition A dagger is a contravariant, identity-on-objects, involutive endofunctor, i.e. a functor $\dagger: \mathbf{D}^{\text {op }} \rightarrow \mathbf{D}$ on a category $\mathbf{D}$ with $X^{\dagger}=X$ on objects and $f^{\dagger \dagger}=f$ on morphisms. We will call $f^{\dagger}$ the adjoint morphism of $f$ or simply the adjoint of $f$. A category that comes equipped with a dagger is then called a dagger category. Together with dagger functors, i.e. functors $F$ satisfying $F\left(f^{\dagger}\right)=F(f)^{\dagger}$, dagger categories form a (large) category DagCat.

3.1.2 The use of daggers, mostly with additional assumptions, dates back to [162, 179]. They have recently been considered independently as axiomatising features in the context of quantum physics in the form portrayed in this chapter ([2, 197], but see also [12, 93, 105] for similar situations with additional assumptions). 
In general, naming concepts after some notation is inferior to finding a descriptive name, since notation is susceptible to change. However, in this case 'involutive' instead of 'dagger' is not flexible enough, because it is not clear what exactly is involuted-objects, morphisms, or both?-as we will see in Example 3.1.8 We follow [199], and use 'dagger' as prefix to signify compatibility, e.g. 'dagger functor', and 'dagger biproduct', 'dagger kernel', in Section 3.2 .

3.1.3 Example Any groupoid is a dagger category, in which the adjoint of a morphism is given by its inverse. In particular, discrete categories are dagger categories. As a non-example, consider a partially ordered set as a category. If it were to have a dagger, then the category would collapse to a discrete one.

3.1.4 Example The category Rel of sets and relations introduced in Example 2.1.6 has a dagger. The adjoint of $R \subseteq X \times Y$ is its relational converse:

$$
R^{\dagger}=\{(y, x) \mid(x, y) \in R\} \subseteq Y \times X .
$$

It is instructive to represent a morphism $R \subseteq X \times Y$ in $\mathbf{R e l}$ as (an equivalence class of ) a pair of maps $\left(X \stackrel{r_{1}}{\longleftarrow} R \stackrel{r_{2}}{\longrightarrow} Y\right.$ ) whose tuple $\left\langle r_{1}, r_{2}\right\rangle: R \rightarrow X \times Y$ of legs is injective. The dagger then becomes

$$
\left(X \stackrel{r_{1}}{\longleftarrow} R \stackrel{r_{2}}{\longrightarrow} Y\right)^{\dagger}=\left(Y \stackrel{r_{2}}{\longleftarrow} R \stackrel{r_{1}}{\longrightarrow} X\right) .
$$

3.1.5 Example The subcategory PInj of Rel consisting of sets and partial injections, introduced in Example 2.1.7, inherits the dagger from Rel. Morphisms in PInj can be represented as spans of monomorphisms with the notational convention

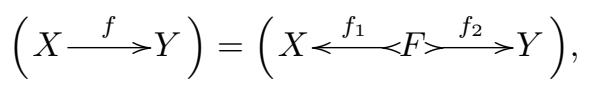

where spans $\left(X \stackrel{f_{1}}{<}<F \stackrel{f_{2}}{\longrightarrow} Y\right)$ and $\left(X \stackrel{g_{1}}{\longleftarrow}<G \stackrel{g_{2}}{\longrightarrow} Y\right)$ are identified if there is an isomorphism $\varphi: F \rightarrow G$ with $g_{i} \circ \varphi=f_{i}$ for $i=1,2$ - like for relations. In this representation, the dagger becomes a simple swap of legs, as before.

3.1.6 Example The category preHilb of pre-Hilbert spaces of Example 2.1.2 has a dagger built in to its definition. Since morphisms are adjointable functions, we can take the dagger of a morphism to be its adjoint. This satisfies $f^{\dagger \dagger}=f$ since

$$
\left\langle f^{\dagger \dagger}(x) \mid y\right\rangle=\left\langle y \mid f^{\dagger \dagger}(x)\right\rangle^{\ddagger}=\left\langle f^{\dagger}(y) \mid x\right\rangle^{\ddagger}=\left\langle x \mid f^{\dagger}(y)\right\rangle=\langle f(x) \mid y\rangle .
$$

In our context, the category Hilb of Hilbert spaces, discussed in Example 2.1.3, is the most important example of a dagger category. Because of 
the completeness of Hilbert spaces, every continuous linear function between Hilbert spaces has a unique adjoint [135, Theorem 2.4.2], determined by the equation

$$
\langle f(x) \mid y\rangle=\left\langle x \mid f^{\dagger}(y)\right\rangle .
$$

The dagger of Hilb descends to the category PHilb of Example 2.1.4, because if $f=z \cdot g$ for some $z \in U(1)$, then

$$
\langle f(x) \mid y\rangle=\bar{z} \cdot\langle g(x) \mid y\rangle=\bar{z} \cdot\left\langle x \mid g^{\dagger}(y)\right\rangle=\left\langle x \mid \bar{z} \cdot g^{\dagger}(y)\right\rangle,
$$

whence $f^{\dagger}=\bar{z} \cdot g^{\dagger}$.

3.1.7 Example Let us mention some examples of dagger categories occurring in the literature without explaining them completely.

Every *-category in the sense of [93] is a dagger category (with certain extra properties). For example, Hilbert modules over a fixed $\mathrm{C}^{*}$-algebra (see Chapter 5) form a dagger category. A more interesting example of a dagger category is the following. Objects are *-morphisms $\rho: A \rightarrow A$ for some fixed unital *algebra $A$, morphisms $\rho \rightarrow \sigma$ are $a \in A$ such that $a=a \cdot \rho(1)$, and $a \cdot \rho(b)=\sigma(b) \cdot a$ for all $b \in A$.

All $n$-dimensional manifolds form the objects of a category whose morphisms are so-called cobordisms. These are $n+1$-dimensional manifolds, whose boundary is the disjoint union of its domain and codomain manifolds. This category is a dagger category; the dagger of a cobordism is its reversal [147].

3.1.8 Example This example describes 'the mother of all dagger categories'.

A functor $*: \mathbf{C}^{\text {op }} \rightarrow \mathbf{C}$ is called involutive when $* \circ *=\mathrm{Id}$. (Daggers are special cases that furthermore fix objects.) Define a category InvAdj as follows. Its objects are pairs $(\mathbf{C}, *)$ of a category with an involution. A morphism $(\mathbf{C}, *) \rightarrow$ $(\mathbf{D}, *)$ is functor $F: \mathbf{C}^{\text {op }} \rightarrow \mathbf{D}$ that has a left adjoint, where two such functors are identified when they are naturally isomorphic. The identity morphism on $(\mathbf{C}, *)$ is the functor $*: \mathbf{C}^{\mathrm{op}} \rightarrow \mathbf{C}$; its left adjoint is $*^{\mathrm{op}}: \mathbf{C} \rightarrow \mathbf{C}^{\mathrm{op}}$. The composition of $F: \mathbf{C}^{\text {op }} \rightarrow \mathbf{D}$ and $G: \mathbf{D}^{\mathrm{op}} \rightarrow \mathbf{E}$ is defined by $G \circ *^{\mathrm{op}} \circ F: \mathbf{C}^{\mathrm{op}} \rightarrow \mathbf{E}$; its left adjoint is $F^{\prime} \circ * \circ G^{\prime}$, where $F^{\prime} \dashv F$ and $G^{\prime} \dashv G$.

Let us show that it is not arbitrary to require a left adjoint instead of a right one. A contravariant functor from $\mathbf{C}$ to $\mathbf{D}$ can be written both as a (covariant) functor $F: \mathbf{C}^{\mathrm{op}} \rightarrow \mathbf{D}$ or as a (covariant) functor $F^{\mathrm{op}}: \mathbf{C} \rightarrow \mathbf{D}^{\mathrm{op}}$. The latter version has a right adjoint $G: \mathbf{D}^{\mathrm{op}} \rightarrow \mathbf{C}$ when $\mathbf{C}(X, G Y) \cong \mathbf{D}(Y, F X)$, or equivalently, when the former version has a left adjoint. (Such an adjunction is also called 'adjoint on the right' [88].) Hence right adjoints of contravariant functors can also be seen as left adjoints. 
InvAdj is a dagger category: the adjoint of a morphism $F: \mathbf{C}^{\mathrm{op}} \rightarrow \mathbf{D}$ is the right adjoint of $F^{\mathrm{op}}: \mathbf{C} \rightarrow \mathbf{D}^{\mathrm{op}}$. Since $G \dashv F$ if and only if $F^{\mathrm{op}} \dashv G^{\mathrm{op}}$, we have $F^{\text {op }} \dashv F^{\dagger}$, so $\left(F^{\dagger}\right)^{\text {op }} \dashv F$, but also $\left(F^{\dagger}\right)^{\text {op }} \dashv F^{\dagger \dagger}$. Because adjoints are unique op to natural isomorphism, thus $[F]=\left[F^{\dagger \dagger}\right]$, as equivalence classes.

3.1.9 Example An orthoposet is a partially ordered set $L$ that comes with a function $\perp: L \rightarrow L$ satisfying $x^{\perp \perp}=x$ and $y^{\perp} \leq x^{\perp}$ when $x \leq y$. They form the objects of a category InvGal. A morphism $L \rightarrow M$ in this category is an antitone Galois connection between $L$ and $M$, i.e. a pair of two functions $f_{*}: L \rightarrow M$ and $f^{*}: M \rightarrow L$, such that $f^{*}(y) \leq x$ iff $y \leq f_{*}(x)$, and $f_{*}(x) \geq f_{*}\left(x^{\prime}\right)$ when $x \leq x^{\prime}$, and $f^{*}(y) \geq f^{*}\left(y^{\prime}\right)$ when $y \leq y^{\prime}$. The identity morphism on $L$ is then given by the pair $(\perp, \perp)$, and the composition of $\left(g_{*}, g^{*}\right)$ and $\left(f_{*}, f^{*}\right)$ is $\left(g_{*} \circ \perp \circ f_{*}, f^{*} \circ \perp \circ g^{*}\right)$.

Thus, InvGal is a full subcategory of InvAdj. We can make it into a dagger category by defining $\left(f_{*}, f^{*}\right)^{\dagger}=\left(f^{*}, f_{*}\right)$.

Before we look at more general examples, let us study some relations between the concrete examples of dagger categories we have seen until now. The following proposition is novel, as far as we know.

3.1.10 Proposition There is a full, faithful, and injective-on-objects dagger functor Sub: Rel $\rightarrow$ InvGal.

Proof On objects, Sub takes a set $X$ to the orthoposet of its subsets $(\mathcal{P}(X), \subseteq)$. If $R \subseteq X \times Y$, then $\operatorname{Sub}(R)$ sends $U \subseteq X$ to $\left\{y \in Y \mid \forall_{x \in U} .(x, y) \notin R\right\}$. Hence $\operatorname{Sub}(R): \mathcal{P}(X)^{\text {op }} \rightarrow \mathcal{P}(Y)$. This assignment is functorial, as for $S \subseteq Y \times Z$ :

$$
\begin{aligned}
\left(\operatorname{Sub}(S) \circ{ }_{\text {InvGal }} \operatorname{Sub}(R)\right)(U) & =(\operatorname{Sub}(S) \circ \perp \circ \operatorname{Sub}(R))(U) \\
& =\operatorname{Sub}(S)\left(\left\{y \in Y \mid \exists_{x \in U} \cdot(x, y) \in R\right\}\right) \\
& =\left\{z \in Z \mid \forall_{x \in U} \forall_{y \in Y} \cdot(x, y) \notin R \vee(y, z) \notin S\right\} \\
& =\left\{z \in Z \mid \forall_{x \in U} \cdot(x, z) \notin S \circ R\right\} \\
& =\operatorname{Sub}(S \circ R)(U) .
\end{aligned}
$$

Indeed, $\operatorname{Sub}(R)$ has a left adjoint (by the adjoint functor theorem) as it preserves infima:

$$
\begin{aligned}
\operatorname{Sub}(R)\left(\bigcup_{i} U_{i}\right) & =\left\{y \in Y \mid \forall_{x \in \cup_{i} U_{i}} \cdot(x, y) \notin R\right\} \\
& =\bigcap_{i}\left\{y \in Y \mid \forall_{x \in U_{i}} \cdot(x, y) \notin R\right\}=\bigcap_{i} \operatorname{Sub}(R)\left(U_{i}\right)
\end{aligned}
$$


Let us show that Sub preserves the dagger.

$$
\begin{aligned}
& U \subseteq \operatorname{Sub}\left(R^{\dagger}\right)(V)=\left\{x \in X \mid \forall_{y \in V} \cdot(x, y) \notin R\right\} \\
\Longleftrightarrow & \forall_{x \in U} \forall_{y \in V} \cdot(x, y) \notin R \\
\Longleftrightarrow & V \subseteq \operatorname{Sub}(R)(U)=\left\{y \in Y \mid \forall_{x \in U} \cdot(x, y) \notin R\right\}
\end{aligned}
$$

So indeed $\operatorname{Sub}\left(R^{\dagger}\right) \dashv \operatorname{Sub}(R)$, so that $\operatorname{Sub}\left(R^{\dagger}\right)=\operatorname{Sub}(R)^{\dagger}$.

Towards faithfulness, let $R, R^{\prime} \subseteq X, Y$ and suppose that $\operatorname{Sub}(R) \neq \operatorname{Sub}\left(R^{\prime}\right)$. Then by definition there is some $U \subseteq X$ such that $\left\{y \mid \forall_{x \in U} \cdot(x, y) \notin R\right\} \neq\{y \mid$ $\left.\forall_{x \in U} .(x, y) \notin R^{\prime}\right\}$. Without loss of generality, there are $U \subseteq X$ and $y \in Y$ with $\forall_{x \in U} .(x, y) \notin R$ and $\exists_{x \in U} .(x, y) \notin R^{\prime}$. In other words, there are $x \in X$ and $y \in Y$ with $(x, y) \in R^{\prime}$ but not $(x, y) \in R$. So $R \neq R^{\prime}$. Thus Sub is faithful.

For fullness, let $f: \mathcal{P}(X) \rightarrow \mathcal{P}(Y)$ be given such that $f\left(\bigcup_{i} U_{i}\right)=\bigcap_{i} f\left(U_{i}\right)$. Define $R=\{(x, y) \mid y \notin f(\{x\})\} \subseteq X \times Y$. Then

$$
\begin{aligned}
f(U) & =f\left(\bigcup_{x \in U}\{x\}\right)=\bigcap_{x \in U} f(\{x\})=\bigcap_{x \in U}\{y \in Y \mid y \in f(\{x\})\} \\
& =\bigcap_{x \in U}\{y \in Y \mid(x, y) \notin R\}=\left\{y \in Y \mid \forall_{x \in U} .(x, y) \notin R\right\}=\operatorname{Sub}(R)(U),
\end{aligned}
$$

establishing that Sub is full.

There is a very similar functor from Hilb to InvGal, as the following proposition shows. This one is not full, however. Both functors will play a prominent role in Chapter 4.

3.1.11 Proposition There is a faithful dagger functor ClSub: Hilb $\rightarrow$ InvGal that is injective on objects.

Proof On objects, define ClSub to take a Hilbert space $X$ to the poset of its closed subspaces. This is indeed an orthoposet, since the involution $M^{\perp}=\{x \in$ $\left.X \mid \forall_{m \in M} \cdot\langle x \mid m\rangle=0\right\}$ satisfies $N^{\perp} \subseteq M^{\perp}$ when $M \subseteq N$. If $f: X \rightarrow Y$ is a bounded linear transformation between Hilbert spaces, then the functor $\operatorname{ClSub}(f): \operatorname{ClSub}(X)^{\mathrm{op}} \rightarrow \operatorname{ClSub}(Y)$ sends a subspace $M \subseteq X$ to $f(M)^{\perp}$. It follows from [174] that the adjoint $f^{\dagger}$ of $f$ is sent to a (left) adjoint of $\mathrm{ClSub}(f)$; see also Theorem 4.4.9 and Theorem 4.4.11 below. To show faithfulness, let $f, f^{\prime}: X \rightrightarrows Y$ and suppose $\operatorname{ClSub}(f) \neq \operatorname{ClSub}\left(f^{\prime}\right)$. Then by definition there is $M \in \operatorname{ClSub}(X)$ such that $f(M)^{\perp} \neq f^{\prime}(M)^{\perp}$. Without loss of generality, there are $M \in \operatorname{ClSub}(X), m \in M$, and $y \in Y$ with $\langle f(m) \mid y\rangle=0 \neq\left\langle f^{\prime}(m) \mid y\right\rangle$. So $f(m) \neq f^{\prime}(m)$, whence $f \neq f^{\prime}$. Thus ClSub is faithful.

3.1.12 Every Hilbert space is automatically a pre-Hilbert space. Conversely, every pre-Hilbert space can be completed to a Hilbert space into which it densely 
embeds, by the process of Cauchy completion [181, I.3]. A bounded adjointable function between pre-Hilbert spaces then extends to a bounded linear function between the resulting Hilbert spaces [181, I.7]. These two functors form a coreflection preHilb ${ }^{\text {bd }} \underset{<}{\prec}$ Hilb.

3.1.13 There is a dagger functor $\ell^{2}: \mathbf{P I n j} \rightarrow$ Hilb. It acts on a set $X$ as

$$
\ell^{2}(X)=\left\{\varphi:\left.X \rightarrow \mathbb{C}\left|\sum_{x \in X}\right| \varphi(x)\right|^{2}<\infty\right\}
$$

which is a Hilbert space under the inner product $\langle\varphi \mid \psi\rangle=\sum_{x \in X} \overline{\varphi(x)} \cdot \psi(x)$ [135].

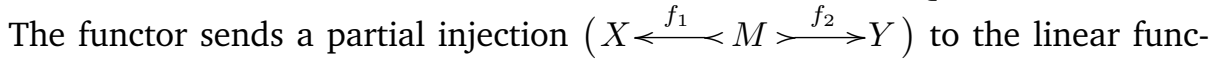
tion $\ell^{2}(f): \ell^{2}(X) \rightarrow \ell^{2}(Y)$ determined informally by $\ell^{2}(f)=\left(\_\right) \circ f^{\dagger}$. Explicitly, $\ell^{2}(f)(\varphi)(y)=\sum_{m \in f_{2}^{-1}(y)} \varphi\left(f_{1}(m)\right)$. This is a well-defined morphism in Hilb:

$$
\begin{aligned}
\sum_{y \in Y}\left|\ell^{2}(f)(\varphi)(y)\right|^{2} & =\sum_{y \in Y}\left|\sum_{m \in f_{2}^{-1}(y)} \varphi\left(f_{1}(m)\right)\right|^{2} \leq \sum_{y \in Y} \sum_{m \in f_{2}^{-1}(y)}\left|\varphi\left(f_{1}(m)\right)\right|^{2} \\
& =\sum_{m \in M}\left|\varphi\left(f_{1}(m)\right)\right|^{2} \leq \sum_{x \in X}|\varphi(x)|^{2}<\infty .
\end{aligned}
$$

To verify that this assignment is functorial, suppose that $\left(Y<^{g_{1}}<N>\stackrel{g_{2}}{\longrightarrow} Z\right.$ ) is another morphisms. Their composition $g \circ f$ is given by $\left(X<_{p_{1}}^{<P>} \stackrel{p_{2}}{\longrightarrow} Z\right)$, where $P=\left\{(m, n) \in M \times N \mid f_{2}(m)=g_{1}(n)\right\}$, with $p_{1}(m, n)=f_{1}(m)$ and $p_{2}(m, n)=g_{2}(n)$. Hence

$$
\begin{aligned}
\left(\ell^{2}(g) \circ \ell^{2}(f)\right)(\varphi)(z) & =\sum_{n \in g_{2}^{-1}(z)} \sum_{m \in f_{2}^{-1}\left(g_{1}(n)\right)} \varphi\left(f_{1}(m)\right)=\sum_{(m, n) \in P, g_{2}(n)=z} \varphi\left(f_{1}(m)\right) \\
& =\sum_{(m, n) \in p_{2}^{-1}(z)} \varphi\left(p_{1}(m, n)\right)=\left(\ell^{2}(g \circ f)\right)(\varphi)(z) .
\end{aligned}
$$

It follows directly from the definition that $\ell^{2}($ id $)=$ id. Hence $\ell^{2}: \mathbf{P I n j} \rightarrow \mathbf{H i l b}$ is indeed a functor. The following calculation shows that it is a dagger functor. For $\varphi \in \ell^{2}(X)$ and $\psi \in \ell^{2}(Y)$ :

$$
\begin{aligned}
\left\langle\ell^{2}(f)(\varphi) \mid \psi\right\rangle_{\ell^{2}(Y)} & =\sum_{y \in Y} \overline{\ell^{2}(f)(\varphi)(y)} \cdot \psi(y)=\sum_{y \in Y} \sum_{m \in f_{2}^{-1}(y)} \overline{\varphi\left(f_{1}(m)\right)} \cdot \psi(y) \\
& =\sum_{m \in M} \overline{\varphi\left(f_{1}(m)\right)} \cdot \psi\left(f_{2}(m)\right)=\sum_{x \in X} \sum_{m \in f_{1}^{-1}(x)} \overline{\varphi(x)} \cdot \psi\left(f_{2}(m)\right) \\
& =\sum_{x \in X} \overline{\varphi(x)} \cdot\left(\sum_{m \in f_{1}^{-1}(x)} \psi\left(f_{2}(m)\right)\right)=\left\langle\varphi \mid \ell^{2}\left(f^{\dagger}\right)(\psi)\right\rangle_{\ell^{2}(X)} .
\end{aligned}
$$


The restriction $\ell^{2}:$ finPInj $\rightarrow$ fdHilb to the category of finite sets and partial injections extends to a functor $\ell^{2}$ : finSet $\rightarrow$ fdHilb, but neither to a functor finRel $\rightarrow$ fdHilb, nor to a functor Set $\rightarrow$ Hilb. This was first noticed in [21], and further studied in [102].

3.1.14 The functor $\ell^{2}$ could be seen as a sort of free functor, as it factors via preHilb $^{\text {bd }}$ by the coreflection of 3.1.12.

Define a functor $F: \mathbf{P I n j} \rightarrow$ preHilb $^{\text {bd }}$ as follows. A set $X$ is mapped to $F(X)=\{\varphi: X \rightarrow \mathbb{C} \mid \operatorname{supp}(\varphi)$ finite $\}$ with $\langle\varphi \mid \psi\rangle_{F X}=\sum_{x \in X} \overline{\varphi(x)} \cdot \psi(x)$. On a morphism $f: X \rightarrow Y$, it is defined as $F f(\varphi)(y)=\sum_{m \in f_{2}^{-1}(y)} \varphi\left(f_{1}(m)\right)$. Then $F f$ is adjointable (with adjoint $F\left(f^{\dagger}\right)$ ), and bounded:

$$
\begin{aligned}
\|F f(\varphi)\| & =\left(\sum_{y \in Y}\left|\sum_{x \in f^{-1}(y)} \varphi(x)\right|^{2}\right)^{\frac{1}{2}} \leq\left(\sum_{y \in Y} \sum_{x \in f^{-1}(y)}|\varphi(x)|^{2}\right)^{\frac{1}{2}} \\
& =\left(\sum_{x \in \operatorname{Dom}(f)}|\varphi(x)|^{2}\right)^{\frac{1}{2}} \leq\left(\sum_{x \in X}|\varphi(x)|^{2}\right)^{\frac{1}{2}}=\|\varphi\| .
\end{aligned}
$$

One could say that $F$ is the free vector space functor that equips its output with the canonical inner product, except that it needs to be restricted to PInj. Finally, $\ell^{2}(X)$ is the Cauchy completion of $F(X)$, and the extension of $F(f)$ is $\ell^{2}(f)$.

We can summarise the above relationships by the following commuting diagram of dagger categories.

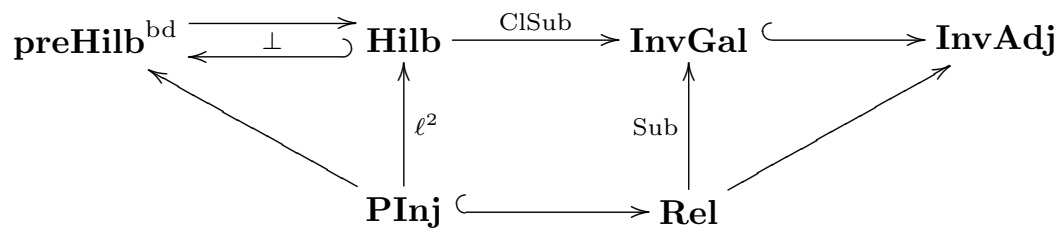

The rest of this section is concerned with more general examples of dagger categories.

3.1.15 Example If $\mathbf{D}$ and $\mathbf{E}$ are dagger categories, then the category $[\mathbf{D}, \mathbf{E}]$ of dagger functors and natural transformations is again a dagger category. The adjoint $\alpha^{\dagger}: G \Rightarrow F$ of a natural transformation $\alpha: F \Rightarrow G$ is determined componentwise, by $\left(\alpha^{\dagger}\right)_{X}=\left(\alpha_{X}\right)^{\dagger}$, as is verified for $f: X \rightarrow Y$ in $\mathbf{D}$ by:

$$
\begin{aligned}
\left(\alpha^{\dagger}\right)_{Y} \circ G(f) & =\left(G(f)^{\dagger} \circ\left(\alpha^{\dagger}\right)_{Y}^{\dagger}\right)^{\dagger}=\left(G\left(f^{\dagger}\right) \circ \alpha_{Y}\right)^{\dagger} \\
& =\left(\alpha_{X} \circ F\left(f^{\dagger}\right)\right)^{\dagger}=\left(\left(\alpha^{\dagger}\right)_{X}^{\dagger} \circ F(f)^{\dagger}\right)^{\dagger}=F(f) \circ\left(\alpha^{\dagger}\right)_{X} .
\end{aligned}
$$


A particularly important special case is the category $[G, \mathbf{H i l b}]$ for a group $G$. An object in this category is a functor $U: G \rightarrow$ Hilb; on objects, $U(*)$ gives a Hilbert space $H_{U}$. On morphisms, $U(g)$ gives a bounded linear transformation $U(g): H_{U} \rightarrow H_{U}$, such that $U(h) \circ U(g)=U(h \circ g)$. The fact that $U$ must preserve the dagger means that $U\left(g^{-1}\right)=U(g)^{\dagger}$. Hence the objects of $[G$, Hilb] of are precisely the unitary representation of the group $G$. Likewise, the morphisms are precisely intertwiners between representations. It could be said that representation theory (see e.g. [166]) is all about the study of the categories of the form $[G$, Hilb $]$.

3.1.16 Definition The construction $\mathbf{C}_{\leftrightarrows}$ of Definition 2.3.5 extends to a functor $\left({ }_{(}\right) \leftrightarrows$ : Cat $\rightarrow$ DagCat as follows. Given a functor $F: \mathbf{C} \rightarrow \mathbf{D}$, define $F_{\leftrightarrows}: \mathbf{C}_{\leftrightarrows} \rightarrow \mathbf{D}_{\leftrightarrows}$ by $F_{\leftrightarrows}(X)=F(X)$ and $F_{\leftrightarrows}\left(f_{\leftarrow}, f_{\rightarrow}\right)=\left(F\left(f_{\leftarrow}\right), F\left(f_{\rightarrow}\right)\right)$.

The following theorem shows that $\mathbf{C}_{\leftrightarrows}$ is the cofree dagger category. The functor $\left(\_\right) \leftrightarrows$ resembles a 'diagonal' Chu construction [20], and is used in reversible computation [126]. There is also a parallel with Corollary 2.3.6; a category $\mathbf{C}$ is self-dual, i.e. $\mathbf{C} \cong \mathbf{C}^{\text {op }}$, if and only if the forgetful functor $\mathbf{C}_{\leftrightarrows} \rightarrow \mathbf{C}$ is an isomorphism.

3.1.17 Theorem The functor $\left(_{-}\right) \leftrightarrows$ is right adjoint to the evident forgetful functor DagCat $\rightarrow$ Cat.

PROOF We are to establish a natural correspondence

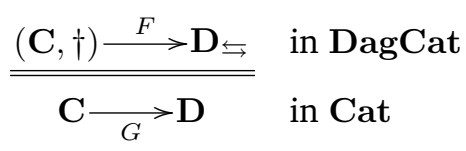

Define the transpose of $F$ by $F^{\vee}(X)=F(X)$ and $F^{\vee}(f)=(F(f))_{\rightarrow}$. This is a well-defined functor. Define the transpose of $G$ by $G^{\wedge}(X)=G(X)$ and $G^{\wedge}(f)=\left(G\left(f^{\dagger}\right), G(f)\right)$. It is a well-defined dagger functor. Moreover

$$
\begin{aligned}
F^{\vee \wedge}(f) & =\left(F^{\vee}\left(f^{\dagger}\right), F^{\vee}(f)\right) \\
& =\left(\left(F\left(f^{\dagger}\right)\right)_{\rightarrow},(F(f))_{\rightarrow}\right) \\
& =\left(\left(F(f)^{\dagger}\right)_{\rightarrow},(F(f))_{\rightarrow}\right) \\
& =\left(F(f)_{\leftarrow}, F(f)_{\rightarrow}\right) \\
& =F(f)
\end{aligned}
$$

and

$$
G^{\wedge \vee}(f)=\left(G^{\wedge}(f)\right)_{\rightarrow}=\left(G\left(f^{\dagger}\right), G(f)\right)_{\rightarrow}=G(f) .
$$


3.1.18 Definition Let a category $\mathbf{C}$ be given. Define a category $\operatorname{Zigzag}(\mathbf{C})$ with the same objects as $\mathbf{C}$, as follows. A morphism from $X$ to $Y$ in $\operatorname{Zigzag}(\mathbf{C})$ consists of a natural number $n=1,2, \ldots$, objects $X_{2}, \ldots, X_{n}$ and $X^{1}, \ldots, X^{n}$ of $\mathrm{C}$, and morphisms

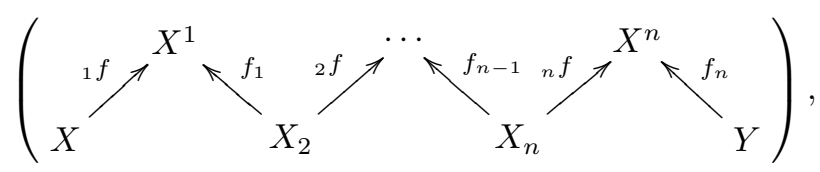

subject to the identifications

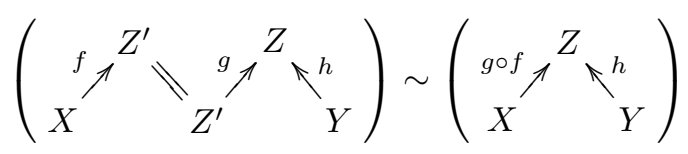

and

$$
\left(\begin{array}{cccc}
f^{f} \nearrow^{Z} & \aleph^{g} & Z^{Z^{\prime}} & \\
X & Z^{\prime} & & Y
\end{array}\right) \sim\left(\begin{array}{ccc}
f_{f} \nearrow^{Z} & \aleph^{g \circ h} \\
X & & Y
\end{array}\right) .
$$

Composition of such 'zigzag' morphisms is defined by juxtaposition. The identity on $X$ is $(X=X=X)$. Thus we have a category $\operatorname{Zigzag}(\mathbf{C})$. In fact, this is a dagger category by

$$
\left({ }_{1} f, f_{1}, \ldots,{ }_{n} f, f_{n}\right)^{\dagger}=\left(f_{n},{ }_{n} f, \ldots, f_{1},{ }_{1} f\right) .
$$

Any morphism in Zigzag $(\mathbf{C})$ can be written in normal form by using (3.1) and (3.2), read from left to right. The normal form of $f$ then has the smallest $n$ possible, and no ${ }_{i} f=$ id or $f_{i}=$ id except perhaps ${ }_{1} f=$ id or $f_{n}=$ id.

For a functor $F: \mathbf{C} \rightarrow \mathbf{D}$, define $\operatorname{Zigzag}(F): \operatorname{Zigzag}(\mathbf{C}) \rightarrow \operatorname{Zigzag}(\mathbf{D})$ by

$$
\begin{aligned}
\operatorname{Zigzag}(F)(X) & =F(X), \\
\operatorname{Zigzag}(F)\left({ }_{1} f, f_{1}, \ldots,{ }_{n}, f_{n}\right) & =\left(F\left({ }_{1} f\right), F\left(f_{1}\right), \ldots, F\left({ }_{n} f\right), F\left(f_{n}\right)\right) .
\end{aligned}
$$

Thus we have a functor Zigzag: Cat $\rightarrow$ DagCat. The following theorem shows that $\mathbf{Z i g z a g}(\mathbf{C})$ is the free dagger category.

3.1.19 Theorem The functor $\operatorname{Zigzag(\_ )~is~left~adjoint~to~the~evident~forgetful~}$ functor DagCat $\rightarrow$ Cat. 
PROOF We are to establish a natural correspondence

$$
\frac{\underset{\mathbf{Z i g z a g}(\mathbf{C}) \stackrel{F}{\longrightarrow}(\mathbf{D}, \dagger)}{\mathbf{C} \underset{G}{\longrightarrow} \mathbf{D}}}{\text { in Cat }} \begin{aligned}
& \text { in DagCat } \\
& \text { in }
\end{aligned}
$$

Define the transpose of $F$ by $F^{\vee}(X)=F(X)$ and $F^{\vee}(f)=F(X \stackrel{f}{\longrightarrow} Y=Y)$; this is a well-defined functor. Define the transpose of $G$ by $G^{\wedge}(X)=G(X)$ and $G^{\wedge}\left({ }_{1} f, f_{1}, \ldots,{ }_{n}, f_{n}\right)=G\left(f_{n}\right)^{\dagger} \circ G\left({ }_{n} f\right) \circ \cdots G\left(f_{1}\right)^{\dagger} \circ G\left({ }_{1} f\right)$; this is a well-defined dagger functor. Moreover

$$
\begin{aligned}
& F^{\vee \wedge}\left(\begin{array}{ccccc}
{ }_{1 f} X^{X^{1}} & & & { }_{n} f_{1} & X^{n} \\
X & X_{2} & \cdots & X_{n} & Y
\end{array}\right) \\
& =\left(F^{\vee}\left(f_{n}\right)\right)^{\dagger} \circ F^{\vee}\left({ }_{n} f\right) \circ \cdots \circ\left(F^{\vee}\left(f_{1}\right)\right)^{\dagger} \circ F^{\vee}\left({ }_{1} f\right)
\end{aligned}
$$

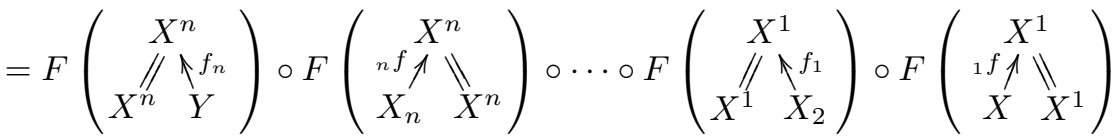

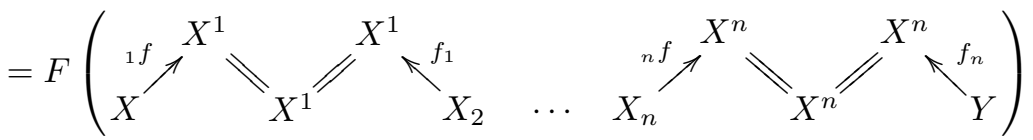

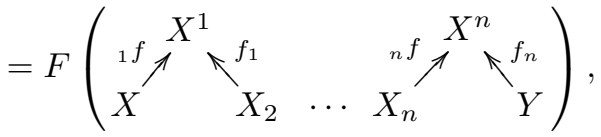

and

$$
G^{\wedge \vee}(f)=G^{\wedge}(X \stackrel{f}{\longrightarrow} Y=Y)=G(\mathrm{id})^{\dagger} \circ G(f)=G(f) .
$$

The categories Rel and PInj are both quotient categories of Zigzag(Set). The previous two theorems can be summarised by the following diagram.

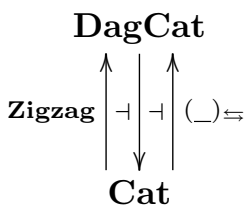

\subsection{Dagger structures}

In a category with an explicit self-duality in the form of a dagger, it makes sense to consider compatibility of the dagger with all kinds of structures. This section 
considers how a dagger interacts with monomorphisms, epimorphisms, tensor products, biproducts, kernels and equalisers.

3.2.1 Definition A monomorphism $f$ in a dagger category is called a dagger mono when $f^{\dagger} \circ f=\mathrm{id}$, i.e. when it is split by its own adjoint. An epimorphism is called a dagger epi when $f \circ f^{\dagger}=$ id, i.e. when its adjoint is dagger mono. A dagger iso is a morphism that is both dagger mono and dagger epi, i.e. a morphism whose adjoint is its inverse.

Some authors transfer terminology from Hilb to any dagger category. For example, dagger monos are also called isometries, since they are precisely the ones that preserve the metric:

$$
\langle f(x) \mid f(y)\rangle=\left\langle x \mid\left(f^{\dagger} \circ f\right)(y)\right\rangle=\langle x \mid y\rangle .
$$

Likewise, dagger isos are also called unitaries. However, in keeping with 3.1.2, we prefer to prefix structures with dagger, with two exceptions that have no obvious equivalent in this terminology. A morphism $f: X \rightarrow X$ is self-adjoint when $f^{\dagger}=f$. A projection is a self-adjoint idempotent morphism, i.e. a morphism $f: X \rightarrow X$ that satisfies $f^{\dagger}=f=f \circ f$.

Let us illustrate the differences between e.g. monos and dagger monos in several of our examples.

3.2.2 Example The requirement that a morphism $R \subseteq X \times Y$ in $\mathbf{R e l}$ is a dagger mono amounts to the equivalence

$$
\exists_{y \in Y} \cdot(x, y) \in R \wedge\left(x^{\prime}, y\right) \in R \quad \Longleftrightarrow \quad x=x^{\prime}
$$

for all $x, x^{\prime} \in X$. This can be split into two statements:

$$
\forall_{x \in X} \cdot \exists_{y \in Y} \cdot(x, y) \in R \quad \text { and } \quad \forall_{x, x^{\prime} \in X, y \in Y} \cdot(x, y) \in R \wedge\left(x^{\prime}, y\right) \in R \Rightarrow x=x^{\prime} .
$$

Hence a dagger mono $R$ is given by a span of the form

$$
\left(X \stackrel{r_{1}}{\ll^{1}} R>^{r_{2}}>Y\right) \text {. }
$$

A dagger epi has the same shape, but with legs exchanged.

Here is an example of a mono which is not a dagger mono. Consider the relation $R \subseteq\{0,1\} \times\{a, b, c\}$ given by $R=\{(0, a),(0, b),(1, b),(1, c)\}$. Clearly, the first leg of $R$ is a surjection, and the second one is neither an injection nor a surjection. To verify that $R$ is monic, suppose $S, T: X \rightarrow\{0,1\}$ satisfy $R \circ S=R \circ T$. If $(x, 0) \in S$, then $(x, a) \in(R \circ S)=(R \circ T)$, so that $(x, 0) \in T$. Similarly, $(x, 1) \in S \Rightarrow(x, 1) \in T$. 
3.2.3 Example Consider a morphism $f=\left(X{f_{1}}^{f_{2}}<F \succ_{f_{2}}^{\longrightarrow} Y\right)$ in PInj. Since $f^{\dagger} \circ f=\left(X<\stackrel{f_{1}}{<F>} \stackrel{f_{1}}{\longrightarrow} X\right)$, the morphism $f$ is a dagger mono if and only if its first leg $f_{1}: F \longmapsto X$ is an isomorphism. We can therefore identify a mono $m: M \longmapsto X$ in Set with the corresponding dagger mono $(M=M>m$ in PInj. In fact, being a mono in PInj and being a dagger mono are precisely the same thing. For if $m: X \rightarrow Y$ is a mono in PInj that is not dagger mono, then there is an $x \in X$ with $x \notin m_{1}(M)$; hence there are different partial injections $f, g: 1 \rightrightarrows X$ such that $m \circ f=m \circ g$, which is a contradiction. Dually, the dagger epis in PInj coincide with all epis.

3.2.4 Example It is not hard to see that the monos in Hilb are precisely the injective continuous linear transformations. On the other hand, we already saw in 3.1.2 that the dagger monos in Hilb are precisely the isometries. The range $\{f(x) \mid x \in X\}$ of an isometry $f: X \rightarrow Y$ is automatically a closed subspace of $Y$ since isometries are injective [10, Proposition 4.5.2].

Dually, dagger epimorphisms in Hilb are automatically surjective [10, Proposition 4.6.1], but the epis in Hilb are precisely the continuous linear transformations whose range is dense. Indeed, if $e: X \rightarrow Y$ satisfies $f \circ e=g \circ e$ for all $f, g: Y \rightarrow Z$ and $e$ has dense range, we can write $y=\lim _{n} e\left(x_{n}\right)$ for $y \in Y$, whence

$$
f(y)=f\left(\lim _{n} e\left(x_{n}\right)\right)=\lim _{n} f\left(e\left(x_{n}\right)\right)=\lim _{n} g\left(e\left(x_{n}\right)\right)=g\left(\lim _{n} e\left(x_{n}\right)\right)=g(y) .
$$

Conversely, suppose that $e: X \rightarrow Y$ is epi. If we denote by $\overline{e(X)}$ the closure of e's range in $Y$, then $Y \overline{e(X)}$ is again a Hilbert space, and the induced projection $p: Y \rightarrow Y / \overline{e(X)}$ is continuous and linear. Then $p \circ e=0 \circ e$, whence $p=0$, and $\overline{e(X)}=Y$.

Here is an example of a morphism that is both mono and epi, but is neither a dagger mono nor a dagger epi. Define $f: \ell^{2}(\mathbb{N}) \rightarrow \ell^{2}(\mathbb{N})$ by $f(\varphi)(n)=\frac{1}{n} \varphi(n)$. It is injective, self-adjoint, and hence also has dense range. But it is not surjective, as $\varphi \in \ell^{2}(\mathbb{N})$ determined by $\varphi(n)=\frac{1}{n}$ is not in its range. Its range therefore cannot be a closed subspace.

3.2.5 Example For any category $\mathbf{C}$, a morphism $f$ of $\mathbf{C}_{\leftrightarrows}$ is a mono precisely when $f_{\rightarrow}$ is mono and $f_{\leftarrow}$ is epi. But $f$ is dagger mono if and only if $\left(f_{\leftarrow}\right) \circ\left(f_{\rightarrow}\right)=$ $\operatorname{id}_{X}$, i.e. when $f_{\leftarrow}$ is a mono that is split by $f_{\rightarrow}$.

Dually, epis and dagger epis have the same characterisation, with constituents exchanged. In particular, $f$ is a dagger isomorphism if and only if $\left(f_{\leftarrow}\right)=$ $\left(f_{\rightarrow}\right)^{-1}$. 
3.2.6 Example For any category $\mathbf{C}$, a morphism $f$ of $\operatorname{Zigzag}(\mathbf{C})$ in normal form is dagger monic if and only if $f_{i}=\mathrm{id}={ }_{i} f$ for all $i=1, \ldots, n$. That is, the only dagger monos are the identities, and dually, the only dagger epis are identities. But there can be more monos than just identities. For example, any $f$ which has a normal form with ${ }_{1} f$ an isomorphism and $f_{1}$ epic in $\mathbf{C}$, is monic in Zigzag $(\mathbf{C})$. One can see this by assuming $f \circ g=f \circ h$ and distinguishing the different cases discerning whether the last legs of $g$ and $h$, and the first leg ${ }_{1} f$ of $f$ are identities. Also, any $f$ in normal form for which ${ }_{1} f$ is monic and non-split epic in $\mathbf{C}$, is monic in $\operatorname{Zigzag}(\mathbf{C})$.

We now move on to the interaction between a dagger and monoidal structures.

3.2.7 Definition A dagger (symmetric) monoidal category is a dagger category $(\mathbf{D}, \dagger)$ that carries a (symmetric) monoidal structure $(\mathbf{D}, \otimes, I)$ for which $(f \otimes$ $g)^{\dagger}=f^{\dagger} \otimes g^{\dagger}$ for all morphisms $f$ and $g$, and whose coherence isomorphisms $\alpha, \rho, \lambda$ (and $\gamma$ ) are dagger isomorphisms.

A dagger (strong) monoidal functor is a functor between dagger monoidal categories that is simultaneously dagger and (strong) monoidal.

3.2.8 Example The symmetric monoidal categories $(\mathbf{R e l}, \times, 1),(\mathbf{P I n j}, \times, 1)$ and $(\mathbf{H i l b}, \otimes, \mathbb{C})$ are all dagger symmetric monoidal categories, when equipped with the daggers covered in Examples 3.1.4, 3.1.5 and 3.1.6, respectively.

3.2.9 Definition An object $X$ of a dagger symmetric monoidal category is said to be dagger compact when it carries a dagger compact structure: this is a compact structure such that $\eta_{X}=\varepsilon_{X}^{\dagger} \circ \gamma_{X^{*}, X}$.

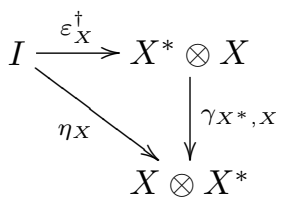

A dagger compact closed category is a dagger symmetric monoidal category whose objects are dagger compact.

3.2.10 In any dagger compact closed category $\mathbf{D}$, the choice-of-duals functor of Proposition 2.6.10 commutes with the dagger [197, Definition 2.9]). Hence there is a covariant functor $\left({ }_{-}\right)_{*}: \mathbf{D} \rightarrow \mathbf{D}$ determined by $X_{*}=X^{*}$ on objects and acting as $f_{*}=f^{* \dagger}=f^{\dagger *}$. In Hilb, this functor maps a morphism to its complex conjugate. 
3.2.11 Example The complex field $\mathbb{C}$ forms a metric space, and hence can be regarded as a $([0, \infty)$-enriched) category, with the metric providing homobjects [155]. By combining this with Example 2.6.5, it turns out that we can regard $\mathbb{C}$ as a dagger compact closed category, as follows. Objects are just complex numbers $x \in \mathbb{C}$. Morphisms $x \rightarrow y$ are $z \in \mathbb{C}$ satisfying $|z|=y-x$. Define identities by $\operatorname{id}_{x}=0$. Composition of $u: x \rightarrow y$ and $v: y \rightarrow z$ is given by $v \circ u=|v|+|u|$. This is indeed well-defined, for

$$
|v \circ u|=|| v|+| u||=|v|+|u|=(z-y)+(y-x)=z-x .
$$

This category has a symmetric monoidal structure, given on objects by $x \otimes y=$ $x+y$, and on morphisms by $u \otimes v=u+v$. The unit for this (strict) monoidal structure is $I=0$. This even provides a symmetric monoidal closed structure, by $y \multimap z=z-y$. Moreover, every object $x$ is compact, by $x^{*}=-x$. Finally, there is a dagger, given by complex conjugation: $u^{\dagger}=\bar{u}$. Thus we can regard the complex numbers as a dagger compact closed category.

3.2.12 Example In the category Rel of sets and relations, every object $X$ is compact: by defining $X^{*}=X$ and

$$
\begin{aligned}
& \eta_{X}=\{(*,(x, x)) \mid x \in X\}, \\
& \varepsilon_{X}=\{((x, x), *) \mid x \in X\},
\end{aligned}
$$

one easily verifies that diagrams (2.18) commute. In fact, this makes $X$ dagger compact. Thus Rel is a dagger compact closed category.

This example can be generalised to the Kleisli category of the monad on Set given by $\mathcal{P}(M \times-)$ for an arbitrary commutative monoid $M$ instead of the trivial monoid. It can also be generalised to the category of relations on an arbitrary regular category [44]. In both the Kleisli categories and the relation categories above, every object is compact.

At first sight, one might expect that the category Sup of complete lattices and supremum-preserving functions is compact, but it is not [20, page 99]. Its largest compact closed subcategory is that of complete atomic Boolean lattices and sup-preserving functions; this category is equivalent to Rel. Coincidentally, Sup is the category of Eilenberg-Moore algebras for the powerset monad, and Rel is the category of Kleisli algebras for that monad.

3.2.13 Continuing 2.6.12, notice that the tensor product $\times$ in Rel does not satisfy the requirement of "behaving bilinearly". Indeed, the left-hand side $\operatorname{Rel}(1, X) \otimes \operatorname{Rel}(1, Y)$ of 2.20$)$ can be identified with $(\mathcal{P}(X) \times \mathcal{P}(Y)) / \sim$, where $\sim$ is the least equivalence relation determined by $(\emptyset, V) \sim(U, \emptyset)$ for $U \subseteq X$ and $V \subseteq Y$. But the right-hand side $\operatorname{Rel}(1, X \times Y)$ is $\mathcal{P}(X \times Y)$, which is of higher 
cardinality in general. This explains why compact objects in Rel can be infinite as sets. This keeps us from calling compact objects "finite objects", which would make sense from the perspective of Examples 2.6.5 and 2.6.6 and the next example.

3.2.14 Example One can extend Example 2.6.6 to Hilb. For a Hilbert space $X$, let $X^{*}$ be the conjugate of the dual space $\operatorname{Hilb}(X, \mathbb{C})=\{f: X \rightarrow \mathbb{C} \mid$ $f$ bounded linear\}, i.e. it has the same additive group as the dual space, but conjugated scalar multiplication. In general, $X^{*} \otimes X$ is isomorphic to the Hilbert space of all Hilbert-Schmidt maps $X \rightarrow X$ [135]. For finite-dimensional $X$, define $\eta_{X}$ by letting 1 correspond to the identity map under this isomorphism and extending linearly and continuously, and define $\varepsilon_{X}$ as the adjoint of $\eta_{X}$. Then diagrams (2.18) commute. Hence any finite-dimensional Hilbert space $X$ is a dagger compact object Hilb.

Since the identity map on $X$ is a Hilbert-Schmidt map if and only if $X$ is finite-dimensional, this recipe for obtaining compact structure on $X$ only works for finite-dimensional $X$. In other words, fdHilb is a full compact closed subcategory of Hilb. Moreover, a full compact closed subcategory of Hilb is necessarily closed by Proposition 2.6.8(d). Since only the Hilbert-Schmidt operators form a Hilbert space again [135], a full compact closed subcategory of Hilb must consist of objects between which all continuous linear functions are automatically Hilbert-Schmidt. That is, the largest full compact closed subcategory of Hilb is fdHilb.

This example can be generalised to the category $[G, \mathbf{H i l b}]$ of unitary representations of a given group $G$, as discussed in Example 3.1.15. The compact objects in that category are precisely the representations with a finite-dimensional carrier space.

3.2.15 Definition A dagger category $(\mathbf{D}, \dagger)$ has (finite) dagger biproducts when it has (finite) biproducts with $\kappa_{i}=\pi_{i}^{\dagger}$. In the algebraic characterisation of Corollary 2.3.6, this is equivalent to $\Delta^{\dagger}=\nabla$ and $u^{\dagger}=n$.

3.2.16 Both $(\mathbf{R e l}, \dagger,+, \emptyset)$ and $(\mathbf{H i l b}, \dagger, \oplus, 0)$ are examples of dagger biproduct categories. The category PInj inherits the dagger symmetric monoidal structure $(+, \emptyset)$ from Rel, but it does not provide biproducts in the former category. Observe, nevertheless, that the 'free vector space functor' $F:$ PInj $\rightarrow$ preHilb $^{\text {bd }}$ from 3.1 .14 preserves this dagger monoidal structure $(+, \emptyset)$. Hence so does the functor $\ell^{2}:$ PInj $\rightarrow$ Hilb. Compare 2.3.15 and 2.3.16. This suggests to study the dagger symmetric monoidal structure $(+, \emptyset)$ on PInj. It is coaffine, i.e. its unit $\emptyset$ is an initial object (and also a final one, in the presence of a dagger). 
3.2.17 Proposition The category finPInj of finite sets and partial injections is the free dagger coaffine category on one object. More precisely, there is a functor 1: $\mathbf{1} \rightarrow$ finPInj such that for every dagger coaffine category $\mathbf{D}$ and every functor $G: \mathbf{1} \rightarrow \mathbf{D}$, there is a unique dagger monoidal functor $D:$ finPInj $\rightarrow \mathbf{D}$ satisfying $D \circ 1 \cong G$, canonically.

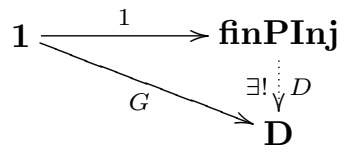

Proof Define the functor 1 by picking a singleton set $\{*\}$ in finPInj. Identify the functor $G$ with an object of the category $\mathbf{D}$. We define the functor $D$ as follows. On objects, $D$ sends a set $X$ to $\oplus_{x \in X} G$. To define its action on morphisms, we use the fact that the category finInj of finite sets and injections is the free coaffine category on one object, i.e. that there is a unique functor $D^{\prime}$ : finInj $\rightarrow \mathbf{D}$ such that $D^{\prime} \circ 1 \cong G$ with the same action on objects as $D$ [176]. Given a partial injection $\left(X<\stackrel{f_{1}}{<}<F>\stackrel{f_{2}}{\longrightarrow} Y\right)$, notice that $F$ must be a finite set, and factor it as $\left(F=F>\stackrel{f_{1}}{\longrightarrow} X\right)^{\dagger}$ followed by $\left(F=F>\stackrel{f_{2}}{\longrightarrow} Y\right)$. Define $D(f)=D^{\prime}\left(f_{2}\right) \circ D^{\prime}\left(f_{1}\right)^{\dagger}$. The desired properties of $D$ now follow from those of $D^{\prime}$.

3.2.18 It is interesting to note that picking $G=\mathbb{C} \in$ fdHilb in the previous proposition results in $D$ being the (restricted) functor $\ell^{2}:$ finPInj $\rightarrow$ fdHilb.

The previous proposition suggests that PInj could be characterised as the free dagger category with 'infinite symmetric monoidal' structure whose unit is initial. Although 'infinite tensor products' have not been studied in the literature, the unbiased categories of [157] could be generalised to fit these requirements. However, we refrain from doing so here.

3.2.19 If a category $\mathbf{C}$ has biproducts, then $\mathbf{C}_{\leftrightarrows}$ has dagger biproducts. The zero object in $\mathbf{C}_{\leftrightarrows}$ is that of $\mathbf{C}$, and $X \oplus Y$ in $\mathbf{C} \leftrightarrows$ is the object $X \oplus Y$ in $\mathbf{C}$. The projection in $\mathbf{C}_{\leftrightarrows}$ is determined by $\pi_{\leftarrow}=\kappa$ and $\pi_{\rightarrow}=\pi$, where the right-hand sides are the biproduct morphisms in $\mathbf{C}$.

The category Zigzag $(\mathbf{C})$ need not have a zero object, even if $\mathbf{C}$ has one; there is still a morphism $(0=0 \longleftarrow 0$ one. Hence Zigzag(_) need not preserve biproducts.

3.2.20 Definition A dagger equaliser of two morphisms $f, g: X \rightrightarrows Y$ is an equaliser of $f, g$ that is a dagger mono. In particular, a dagger kernel of $f: X \rightarrow$ 
$Y$ is a dagger equaliser of $f$ and $0: X \rightarrow Y$ :

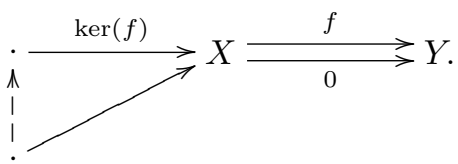

There is a subtlety in saying that a category has dagger equalisers: equalisers are closed under precomposition with arbitrary isomorphisms, but dagger equalisers are only closed under precomposition with dagger isomorphisms. Hence we say that a dagger category has dagger equalisers when it has (finite) equalisers and for every equaliser there is a dagger mono that is isomorphic to it. The same goes for dagger kernels. A dagger category that has dagger equalisers is called a dagger equaliser category, and a dagger category that has (a zero object and) dagger kernels is called a dagger kernel category. The latter form a category DagKerCat, the morphisms of which are dagger functors that preserve the zero object and kernels. In diagrams, we draw dagger kernels as $\triangleright$.

3.2.21 If a dagger category has limits of a certain symmetric shape, then it also has colimits of that shape. For if $D: \mathbf{J} \rightarrow \mathbf{D}$ is a diagram in a dagger category, we can consider the reversed diagram $\dagger \circ D^{\mathrm{op}}: \mathbf{J}^{\mathrm{op}} \rightarrow \mathbf{D}$. If the latter diagram has a limit cone $l_{j}: L \rightarrow D(j)$, then $l_{j}^{\dagger}: D(j) \rightarrow L$ is a colimit cone for the original diagram.

Thus, if a dagger category has dagger equalisers, it also has dagger coequalisers, i.e. coequalisers that can be chosen to be dagger epi. And if a dagger category has dagger kernels, it also has dagger cokernels, i.e. cokernels that can be chosen to be dagger epi, by coker $(f)=\operatorname{ker}\left(f^{\dagger}\right)^{\dagger}$. In diagrams, we draw dagger cokernels as $\longrightarrow$.

3.2.22 Example Perhaps the best example of dagger kernels can be given in categories with dagger biproducts. Then $\operatorname{ker}\left(\pi_{1}\right)=\kappa_{2}$ and $\operatorname{ker}\left(\pi_{2}\right)=\kappa_{1}$, almost by definition. Certainly $\pi_{1} \circ \kappa_{2}=0$, and if $\pi_{1} \circ f=0$ for some $f: X \rightarrow X_{1} \oplus X_{2}$, then $f=\left\langle\kappa_{1} \circ \pi_{1} \circ f, \kappa_{2} \circ \pi_{2} \circ f\right\rangle=\left\langle 0, \kappa_{2} \circ \pi_{2} \circ f\right\rangle=\kappa_{2} \circ\left\langle 0, \pi_{2} \circ f\right\rangle$, so that $f$ indeed factors uniquely through $\kappa_{2}$. Similarly, $\operatorname{coker}\left(\kappa_{1}\right)=\pi_{2}$ and $\operatorname{coker}\left(\kappa_{2}\right)=\pi_{1}$.

3.2.23 Example The category Rel has dagger kernels. For an arbitrary morphism $R: X \rightarrow Y$ one takes $\operatorname{ker}(R)=\{x \in X \mid \neg \exists y \in Y .(x, y) \in R\}$ with map $k: \operatorname{ker}(R) \rightarrow X$ in Rel given by $k=\{(x, x) \mid x \in \operatorname{ker}(R)\}$. Clearly, $R \circ k=0$. And if $S: Z \rightarrow X$ satisfies $R \circ S=0$, then $\neg \exists x \in X .(x, y) \in R \wedge(z, x) \in S$, for all $z \in Z$ and $y \in Y$. This means that $(z, x) \in S$ implies there is no $y$ with $(x, y) \in R$. Hence $S$ factors through the kernel $k$. Kernels are therefore of the 
following form:

$$
\left({ }_{K}{ }^{K} \searrow_{X}\right) \text { with } K=\left\{x \in X \mid \neg \exists_{y \in Y} .(x, y) \in R\right\} .
$$

Since relations of this form are dagger mono by Example 3.2.2, Rel has dagger kernels. But not every dagger mono is a dagger kernel. Notice that kernels correspond to subsets of $X$.

However, Rel does not have dagger equalisers, since it even lacks equalisers. To see this, consider the sets $X=\{0,1\}$ and $Y=\{0\}$, and the parallel relations $R=X \times Y$ and $S=\{(0,0)\} \subseteq X \times Y$. Their equaliser must be contained in $T=\{(0,0)\} \subseteq\{0\} \times X$. Now $T^{\prime}=\{0\} \times X$ also satisfies $R \circ T^{\prime}=S \circ T^{\prime}$, but does not factor through any subrelation of $T$.

3.2.24 Example The category PInj also has dagger kernels. For the description of the kernel of a morphism $f=\left(X \stackrel{f_{1}}{\longleftarrow} F \stackrel{f_{2}}{\longleftrightarrow} Y\right)$ we shall use the ad hoc notation $\neg f_{1}: \neg_{1} F \longmapsto X$ for the complement of the first leg $f_{1}: F \longmapsto X$ as a subset, yielding

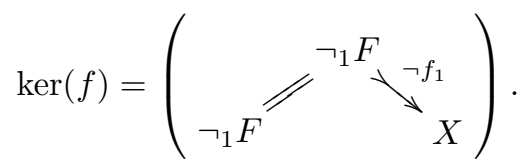

This satisfies $f \circ \operatorname{ker}(f)=0$, and is a dagger mono by construction. Notice that in PInj, too, kernels correspond to subsets. But in PInj, unlike in Rel, every dagger mono is a dagger kernel by Example 3.2.3. Seeing that PInj even has dagger equalisers is easier in the notation of Example 2.1.7. The equaliser of $f, g: X \rightrightarrows Y$ is the inclusion of

$$
\{x \in X \mid x \notin(\operatorname{dom}(f) \cup \operatorname{dom}(g)) \vee(x \in(\operatorname{dom}(f) \cap \operatorname{dom}(g)) \wedge f(x)=g(x))\}
$$

into $X$. Indeed, for $g=0$ this reduces to the above $\operatorname{ker}(f)$.

3.2.25 Example The category Hilb has dagger equalisers. The equaliser of morphisms $f, g: X \rightrightarrows Y$ is the inclusion of the subspace $\{x \in X \mid f(x)=g(x)\}$ into $X$. Since this subspace is always closed, its inclusion is a dagger mono by Example 3.2.4 As a special case, Hilb also has dagger kernels. But we can also derive dagger equalisers from dagger kernels, since the equaliser of $f$ and $g$ is the kernel of $g-f$.

Every dagger mono $m: X \longmapsto Y$ in Hilb is a dagger kernel, namely of the orthogonal projection onto the orthocomplement of its range $\{m(x) \mid x \in X\}$ (see Example 4.2.4). 
3.2.26 The functor $\ell^{2}:$ PInj $\rightarrow$ Hilb preserves dagger kernels: for a partial

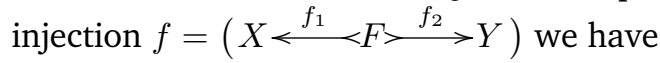

$$
\begin{aligned}
\operatorname{ker}\left(\ell^{2}(f)\right) & =\left\{\varphi \in \ell^{2}(X) \mid \ell^{2}(f)(\varphi)=0\right\} \\
& =\left\{\varphi \in \ell^{2}(X) \mid \forall_{y \in Y} \cdot \sum_{u \in f_{2}^{-1}(y)} \varphi\left(f_{1}(u)\right)=0\right\} \\
& =\left\{\varphi \in \ell^{2}(X) \mid \forall \forall_{u \in F} \cdot \varphi\left(f_{1}(u)\right)=0\right\} \\
& =\left\{\varphi \in \ell^{2}(X) \mid \operatorname{supp}(\varphi) \subseteq \neg_{1} F\right\} \\
& =\ell^{2}\left(\neg_{1} F\right) \\
& =\ell^{2}(\operatorname{ker}(f)) .
\end{aligned}
$$

However, $\ell^{2}$ does not preserve dagger equalisers. For a counterexample, take $X=\{0,1\}, Y=\{a\}$, and let $f, g: X \rightrightarrows Y$ be the partial injections $f=\{(0, a)\}$ and $g=\{(1, a)\}$. Their equaliser in PInj is $\emptyset$. But

$$
\begin{aligned}
\mathrm{eq}\left(\ell^{2}(f), \ell^{2}(g)\right) & =\left\{\varphi \in \ell^{2}(X) \mid \ell^{2}(f)(\varphi)=\ell^{2}(g)(\varphi)\right\} \\
& =\left\{\varphi \in \ell^{2}(X) \mid \forall y \in Y \cdot \sum_{u \in f_{2}^{-1}(y)} \varphi\left(f_{1}(u)\right)=\sum_{v \in g_{2}^{-1}(y)} \varphi\left(g_{1}(v)\right)\right\} \\
& =\{\varphi:\{0,1\} \rightarrow \mathbb{C} \mid \varphi(0)=\varphi(1)\} \\
& \cong \mathbb{C} .
\end{aligned}
$$

Hence eq $\left(\ell^{2}(f), \ell^{2}(g)\right) \cong \mathbb{C} \nsucceq\{\emptyset\}=\ell^{2}(\operatorname{eq}(f, g))$.

3.2.27 Example Dagger kernels in Hilb descend to PHilb. More precisely, the kernel $\operatorname{ker}(f)=\{x \in X \mid f(x)=0\}$ of a morphism $f: X \rightarrow Y$ is well-defined, for if $f=z \cdot f^{\prime}$ for some $z \in U(1)$, then

$$
\operatorname{ker}(f)=\left\{x \in X \mid z \cdot f^{\prime}(x)=0\right\}=\left\{x \in X \mid f^{\prime}(x)=0\right\}=\operatorname{ker}\left(f^{\prime}\right) .
$$

However, dagger equalisers do not descend from Hilb to PHilb. Defining the equaliser of $f, g: X \rightrightarrows Y$ as (the inclusion of) $\{x \in X \mid f(x)=g(x)\}$ is not respected by the equivalence relation. For if $f=u \cdot f^{\prime}$ and $g=v \cdot g^{\prime}$ for some $u, v \in U(1)$, then there is no reason why $f(x)=u \cdot f^{\prime}(x)=v \cdot g^{\prime}(x)=g(x)$ should hold if and only if $f^{\prime}(x)=g^{\prime}(x)$, except when $u=v$.

3.2.28 Example If $\mathbf{C}$ and $\mathbf{D}$ are dagger categories, then the dagger functor category $[\mathbf{C}, \mathbf{D}]$ has dagger kernels whenever $\mathbf{D}$ does. The kernel is computed objectwise. Let $F, G: \mathbf{C} \rightrightarrows \mathbf{D}$ and $\alpha: F \Rightarrow G$ be given. The assignment $K(X)=\operatorname{ker}\left(\alpha_{X}\right)$ on objects defines a functor $K: \mathbf{C} \rightarrow \mathbf{D}$ that acts on mor- 
phisms as follows. For $f: X \rightarrow Y$ in $\mathbf{C}$, we have

$$
\alpha_{Y} \circ\left(F f \circ \operatorname{ker}\left(\alpha_{X}\right)\right)=G f \circ \alpha_{X} \circ \operatorname{ker}\left(\alpha_{X}\right)=G f \circ 0=0,
$$

inducing a morphism $K f: K X \rightarrow K Y$. In a diagram:

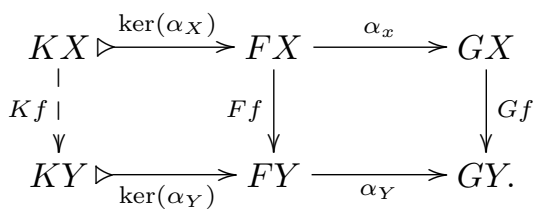

This also shows that $\operatorname{ker}\left(\alpha_{X}\right)$ defines a natural transformation $K \Rightarrow F$. It is the kernel of $\alpha$ in $[\mathbf{C}, \mathbf{D}]$. Similarly, $[\mathbf{C}, \mathbf{D}]$ has dagger equalisers when $\mathbf{D}$ does.

3.2.29 Example For any category $\mathbf{C}$, kernels in the cofree dagger category $\mathbf{C}_{\leftrightarrows}$ are given by $\operatorname{ker}(f)=\left(\operatorname{ker}\left(f_{\rightarrow}\right)\right.$, $\left.\operatorname{coker}\left(f_{\leftarrow}\right)\right)$, if the latter pair exists and coincides in $\mathbf{C}$.

The free dagger functor Zigzag(_) need not preserve zero objects, as seen in 3.2.19, and hence also need not preserve kernels.

\subsection{Quantum key distribution}

We have now gathered enough categorical structure to consider an application. As an intermezzo to the theory, this section models a certain quantum key distribution protocol and prove its correctness. This exemplifies the point of view that compact objects model a (one-time) quantum communication channel, as recognised by [2, 72, 101].

3.3.1 Quantum key distribution is the name for a collection of protocols that provide two parties (traditionally called Alice and Bob) with a shared binary string, unknowable to anyone else, using a quantum channel between them. Moreover, such a scheme must be provably secure by the laws of nature, i.e. its security may not depend on any unsolved or computationally unfeasible mathematical problems. Like Whitfield Diffie and Martin Hellmann's [70], such a protocol regulates key distribution, but gives no guarantee about authenticity of the two parties involved. The best-known protocol in this family is that of Charles Bennett and Giles Brassard [27], which essentially relies on Bell's inequalities and the law of large numbers to provide secure keys. There are several improvements upon this protocol. In particular, Arthur Ekert [80] has developed a very nice simplification of the Bennett-Brassard protocol, which is outlined in Figure 3.3.1. As Bell's inequalities provide a means to verify that two qubits 
1. Alice and Bob agree upon 3 measurements $m_{1}, m_{2}, m_{3}$.

2. Alice secretly chooses $a_{i} \in\{1,2,3\}$ for $i=1, \ldots, 3$ n randomly, Bob secretly chooses $b_{i} \in\{1,2,3\}$ for $i=1, \ldots, 3 n$ randomly.

3. They share $3 n$ fresh qubit-pairs prepared in the Bell state $\frac{|01\rangle-|10\rangle}{\sqrt{2}}$. We denote these by $\left(x_{i}^{a}, x_{i}^{b}\right)_{i=1, \ldots, 3 n}$.

4. Alice measures $x_{i}^{a}$ with $m_{a_{i}}$ to obtain $c_{i}$ for $i=1, \ldots, 3 n$. Bob measures $x_{i}^{b}$ with $m_{b_{i}}$ to obtain $c_{i}^{\prime}$ for $i=1, \ldots, 3 n$.

5. Alice publicly announces $a_{i}$. Bob publicly announces $b_{i}$. Thus they determine $I=\left\{i \in\{1, \ldots, 3 n\} \mid a_{i} \neq b_{i}\right\}$. With large probability $\# I \leq n$; if not, go to step 2 .

6. Alice publicly announces $c_{i}$ for $i \in I$. Bob publicly announces $c_{i}^{\prime}$ for $i \in I$.

With large probability, $c_{i}$ and $c_{i}^{\prime}$ are sufficiently correlated by Bell's inequality for $i \in I$; if not, go to step 2 .

7. Alice uses $c_{j}$ for $j \in\{1, \ldots, 3 n\} \backslash I$ as her key bits. Bob uses $1-c_{j}^{\prime}$ for $j \in\{1, \ldots, 3 n\} \backslash I$ as his key bits.

Figure 3.3.1: Ekert's quantum protocol to obtain a $2 n$-bits shared secret key [80].

are 'sufficiently correlated', potential eavesdroppers can be detected with large probability. The law of large numbers thus ensures that this protocol works (up to a negligible probability that can be specified in advance).

3.3.2 We need to distinguish between correctness and security. A quantum key distribution protocol is correct if in each and every run both parties end up with the same key (which may, of course, depend on the particular run), i.e. when $c_{j}=1-c_{j}^{\prime}$ for all $j \in\{1, \ldots, 3 n\} \backslash I$ and every choice of $m_{i}, a_{i}$ and $b_{i}$ in Figure 3.3.1. It is secure when a potential eavesdropper cannot learn any of the key bits. In this instance, the security relies on Bell's inequalities. Hence in this case one could say that correctness is a qualitative notion, whilst security is a quantitative one. Because the very purpose of our categorical approach is to abstract away from quantitative details, we will focus on correctness, and forget about the classical calculation in steps 5 and 6 . Our point is just to show that compact objects are capable of modeling protocols involving quantum communication via a pair of entangled qubits. 
One final ingredient that we must consider before modeling the above protocol categorically, is classical communication.

3.3.3 A compact structure can be seen as a single instance of quantum communication. In the traditional formalism of quantum information theory [173], the preparation of a pair of qubits in a so-called Bell state is precisely equivalent to the compact structure on $X=\mathbb{C}^{2}$ described in Example 3.2.14. The fact that performing an operation on $X$ also affects $X^{*}$ is reflected by the existence of $\varepsilon$ and commutativity of diagrams (2.18): thus we can regard id: $X \rightarrow X$ or its name $\eta=\ulcorner\operatorname{id}\urcorner: I \rightarrow X^{*} \otimes X$ as a quantum channel capable of a onetime quantum communication. Graphical languages make this fact a bit more intuitive [2, 197, 200].

Let us now consider classical communication. The following definition of classical structure, which is due to Bob Coecke and Duško Pavlović [49-52], counterfactually exploits the fact that quantum data cannot be cloned or forgotten [69, 216]. In contrast to a compact structure, which accommodates a single instance of communication, a classical structure accommodates any finite amount of communication.

3.3.4 Definition A classical structure in a dagger symmetric monoidal category is a commutative comonoid $(A, \delta: A \rightarrow A \otimes A, \nu: A \rightarrow I)$ such that $\delta$ is a dagger mono satisfying the Frobenius condition:

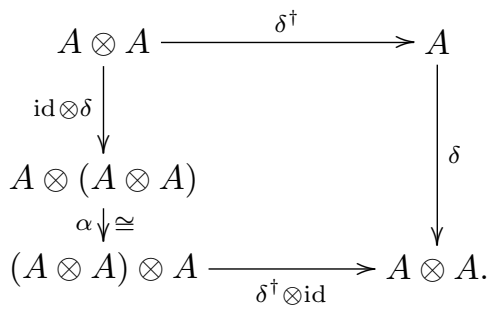

(This condition is named after Ferdinand G. Frobenius, who first studied the requirement that $A \cong A^{*}$ as right $A$-modules for a ring $A$ in the context of group representations [147, 2.2.19])

3.3.5 Example In the category fdHilb of finite-dimensional Hilbert spaces, any choice of an orthonormal basis $\left(e_{1}, \ldots, e_{n}\right)$ for an object $A$ provides it with a classical structure by (linear extension of) the assignments

$$
\begin{aligned}
& \delta\left(e_{i}\right)=e_{i} \otimes e_{i}, \\
& \nu\left(e_{i}\right)=1 .
\end{aligned}
$$


In fact, all classical structures in fdHilb are of this form [53], so in this case we can think of a classical structure as an object equipped with a choice of orthonormal basis.

Each choice of biproduct in the same category provides a standard choice of orthonormal basis for any object $A$ : since there is a dagger isomorphism $A \cong \bigoplus_{i=1}^{n} \mathbb{C}$ for some $n \in \mathbb{N}$, the standard basis for $\mathbb{C}^{n}$ transfers to $A$ by [51, Lemma 3.7]. The comparison with Theorem 2.3.2 then lets one regard classical structure as 'partial biproduct structure'.

The following lemma shows that classical communication can be seen as an extra structure on a compact object-namely a special kind of comonoid, modeling copying and deleting of classical information.

3.3.6 Lemma [52] If $(\delta, \nu)$ is a classical structure for an object $A$ in a symmetric monoidal category, then $A^{*}=A, \eta=\delta \circ \nu^{\dagger}$ and $\varepsilon=\nu \circ \delta^{\dagger}$ provide compact structure for $A$.

Classical and quantum structures are related by measurement. The next definition is an unsophisticated version of the notion defined in [50]. For example, it disregards probabilities, that are of major importance in quantum information theory. Nevertheless, it suffices for our purposes.

3.3.7 Definition A measurement of a compact object $X$ with respect to a classical structure $(A, \delta, \nu)$ in a dagger symmetric monoidal category is a dagger epi $m: X \rightarrow A$.

3.3.8 We model the protocol of Figure 3.3.1 as follows. The qubit is modeled by a compact object $X$, and a classical structure $A$ represents the bit. Let us denote the measurements that step 1 provides by $m_{i}: X \rightarrow A$. The quantum communication channel is set up by the morphism $\eta: I \rightarrow X^{*} \otimes X$, after which Alice receives and can only act upon $X^{*}$, and Bob's operations are restricted to $X$. Alice's procedure for obtaining the $c_{i}$ that might eventually become her key bits, then, is the morphism

$$
X^{*} \otimes X \stackrel{\left(m_{i}\right)_{*} \otimes m_{i}}{\longrightarrow} A^{*} \otimes A \stackrel{\nu \otimes \mathrm{id}}{\longrightarrow} I \otimes A \stackrel{\cong}{\longrightarrow} A .
$$

Likewise, Bob's procedure is

$$
X^{*} \otimes X \stackrel{\left(m_{i}\right)_{*} \otimes m_{i}}{\longrightarrow} A^{*} \otimes A \stackrel{\text { id } \otimes \nu}{\longrightarrow} A^{*} \otimes I \stackrel{\cong}{\longrightarrow} A .
$$

We are now in a position to prove the correctness of the protocol categorically, i.e. to prove that Alice and Bob in fact end up with equal key bits, without assuming anything about the measurements $m_{i}$ or the external choices of $a_{i}$ and 
$b_{i}$. It suffices to prove this for each individual key bit that arises from Alice and Bob using the same measurement, because step 7 discards the other bits. Hence the correctness of the protocol comes down to the correctness of each of its rounds, and is therefore proved by the following theorem.

3.3.9 Theorem The following diagram commutes for any measurement $m: X \rightarrow$ A:

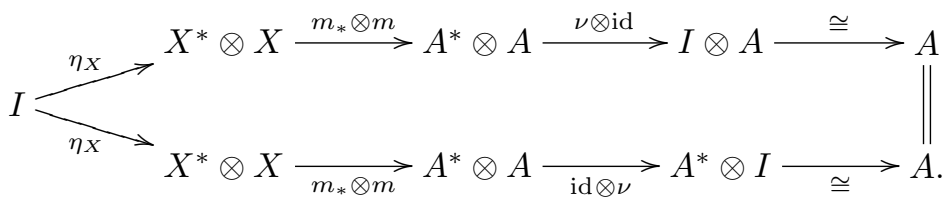

Proof First, notice that

$$
\left(m_{*} \otimes m\right) \circ \eta_{X}=\left(m_{*} \otimes m\right) \circ\left\ulcorner\operatorname{id}_{X}\right\urcorner \stackrel{2.19}{=}\left(m_{*} \otimes \mathrm{id}\right) \circ\ulcorner m\urcorner \stackrel{2.19}{=}\left\ulcorner m \circ m^{\dagger}\right\urcorner=\left\ulcorner\operatorname{id}_{A}\right\urcorner .
$$

since $m \circ m^{\dagger}=$ id by Definition 3.3.7. The commutativity of the above diagram is then established by the following calculation that uses Lemma 3.3.6.

$$
\begin{aligned}
(\nu \otimes \mathrm{id}) \circ\left(m_{*} \otimes m\right) \circ \eta_{X} & =(\nu \otimes \mathrm{id}) \circ\left\ulcorner\mathrm{id}_{A}\right\urcorner \\
& =(\nu \otimes \mathrm{id}) \circ \delta \circ \nu^{\dagger} \\
& =(\mathrm{id} \otimes \nu) \circ \delta \circ \nu^{\dagger} \\
& =(\mathrm{id} \otimes \nu) \circ\left\ulcorner\mathrm{id}_{A}\right\urcorner \\
& =(\text { id } \otimes \nu) \circ\left(m_{*} \otimes m\right) \circ \eta_{X} .
\end{aligned}
$$

\subsection{Factorisation}

Dagger kernel categories have a feature that will turn out to be important later: every morphism can be factored as a dagger cokernel followed by a morphism of a type to be detailed later, followed by a dagger kernel. This section studies this phenomenon, starting with some basic observations.

3.4.1 Lemma In a dagger kernel category, for any morphism $f$,
(a) $\operatorname{ker}(X \stackrel{0}{\longrightarrow} Y)=(X \stackrel{\text { id }}{\longrightarrow} X)$;
(b) $\operatorname{ker}(\operatorname{ker}(f))=0$;
(c) $\operatorname{ker}(\operatorname{coker}(\operatorname{ker}(f)))=\operatorname{ker}(f)$. 
Proof Since (a) and (b) are elementary, we only prove (c). For an arbitrary $f: X \rightarrow Y$, consider the following diagram.

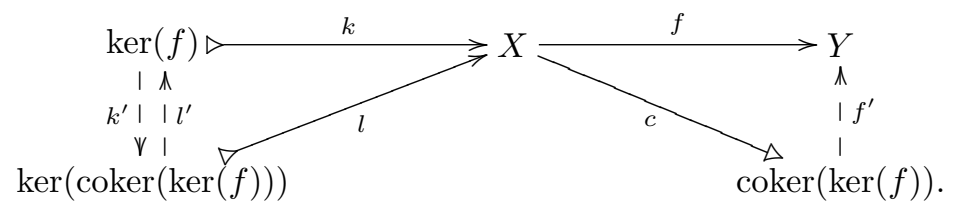

By construction, $f \circ k=0$ and $c \circ k=0$. Hence there are $f^{\prime}$ and $k^{\prime}$ as indicated. Since $f \circ l=f^{\prime} \circ c \circ l=f^{\prime} \circ 0=0$ one gets $l^{\prime}$. Hence the kernels $l$ and $k$ are equal as subobjects.

3.4.2 Lemma For monos $m, n$ in a dagger category with $m \leq n$, say via $m=n \circ \varphi$ :

(a) if $m, n$ are dagger mono, then so is $\varphi$;

(b) if $m$ is a kernel, then so is $\varphi$.

Proof If $m=n \circ \varphi$ and $m, n$ are dagger mono, then

$$
\varphi^{\dagger} \circ \varphi=\left(n^{\dagger} \circ m\right)^{\dagger} \circ \varphi=m^{\dagger} \circ n \circ \varphi=m^{\dagger} \circ m=\mathrm{id} .
$$

And if $m=\operatorname{ker}(f)$, then $\varphi=\operatorname{ker}(f \circ n)$, since: (1) $f \circ n \circ \varphi=f \circ m=0$, and (2) if $f \circ n \circ g=0$, then there is a unique $\psi$ with $m \circ \psi=n \circ g$; but then $\varphi \circ \psi=g$, since $n$ is monic.

3.4.3 Proposition In a dagger kernel category, pullbacks of kernels exist and are kernels again. Explicitly, given a kernel $n$ and map $f$, one obtains a pullback

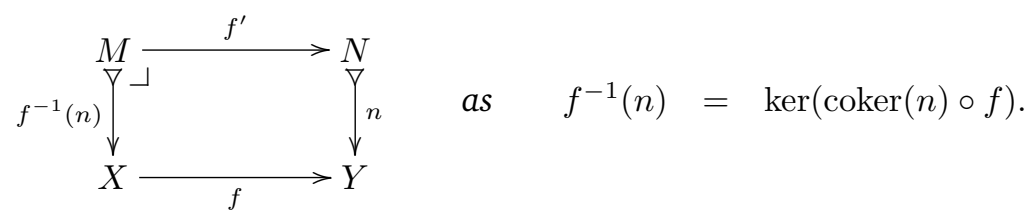

If $f$ is a dagger epi, then so is $f^{\prime}$.

PROOF For convenience write $m=f^{-1}(n)=\operatorname{ker}(\operatorname{coker}(n) \circ f)$. By construction, $\operatorname{coker}(n) \circ f \circ m=0$, so that $f \circ m$ factors through $\operatorname{ker}(\operatorname{coker}(n))=n$, say via $f^{\prime}: M \rightarrow N$ with $n \circ f^{\prime}=f \circ m$, as in the diagram. This yields a pullback. For if $a: Z \rightarrow X$ and $b: Z \rightarrow N$ satisfy $f \circ a=n \circ b$, then

$$
\operatorname{coker}(n) \circ f \circ a=\operatorname{coker}(n) \circ n \circ b=0 \circ b=0,
$$


so that there is a unique map $c: Z \rightarrow M$ with $m \circ c=a$. Then $f^{\prime} \circ c=b$ because $n$ is monic.

In case $f$ is dagger epic, $f \circ f^{\dagger} \circ n=n$. Hence there is a morphism $f^{\prime \prime}$ making following diagram commute, as the right square is a pullback:

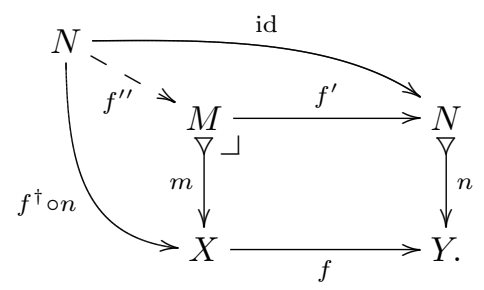

Then $f^{\prime \prime}=m^{\dagger} \circ m \circ f^{\prime \prime}=m^{\dagger} \circ f^{\dagger} \circ n=f^{\prime \dagger} \circ n^{\dagger} \circ n=f^{\prime \dagger}$. Hence $f^{\prime}$ is dagger epic, too.

3.4.4 Lemma In a dagger kernel category, kernels are closed under composition, and hence so are cokernels.

Proof We shall prove the result for cokernels, because it uses the pullback of the previous proposition. Assume we have (composable) cokernels $e, d$; we wish to show $e \circ d=\operatorname{coker}(\operatorname{ker}(e \circ d))$. First notice, using Proposition 3.4.3,

$$
\operatorname{ker}(e \circ d)=\operatorname{ker}(\operatorname{coker}(\operatorname{ker}(e)) \circ d)=d^{-1}(\operatorname{ker}(e)),
$$

yielding a pullback

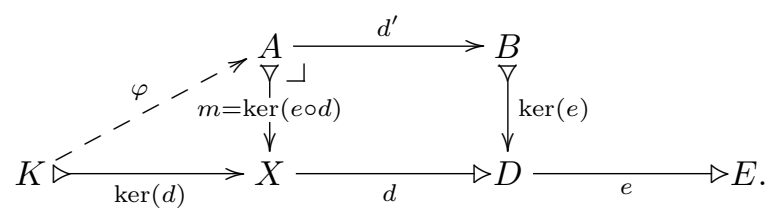

We intend to prove $e \circ d=\operatorname{coker}(m)$. Clearly,

$$
e \circ d \circ m=e \circ \operatorname{ker}(e) \circ d^{\prime}=0 \circ d^{\prime}=0 .
$$

And if $f: X \rightarrow Y$ satisfies $f \circ m=0$, then $f \circ \operatorname{ker}(d)=f \circ m \circ \varphi=0$, so because $d=\operatorname{coker}(\operatorname{ker}(d))$ there is $f^{\prime}: D \rightarrow Y$ with $f^{\prime} \circ d=f$. But then

$$
f^{\prime} \circ \operatorname{ker}(e) \circ d^{\prime}=f^{\prime} \circ d \circ m=f \circ m=0 .
$$

Then $f^{\prime}$ oker $(e)=0$, because $d^{\prime}$ is dagger-epi because $d$ is (see Proposition 3.4.3). This finally yields $f^{\prime \prime}: E \rightarrow Y$ with $f^{\prime \prime} \circ e=f^{\prime}$. Hence $f^{\prime \prime} \circ e \circ d=f$. 
As the following notion does not seem to have an established terminology, we introduce our own.

3.4.5 Definition In a category with a zero object, a morphism $m$ is called a zero mono if $m \circ f=0$ implies $f=0$ for each $f$. Dually, $e$ is zero epi if $f \circ e=0$ implies $f=0$. In diagrams we write $>0>$ for zero monos and $\longrightarrow 0 \gg$ for zero epis.

Clearly, any epi is a zero epi, since $f \circ e=0=0 \circ e$ implies $f=0$ if $e$ is epic. The following lemma shows that the converse also holds in the presence of dagger equalisers.

3.4.6 Lemma In a dagger equaliser category where every dagger mono is a kernel, zero epis are ordinary epis.

Proof Assume a zero epi $e: E \rightarrow X$ with two maps $f, g: X \rightrightarrows Y$ satisfying $f \circ e=g \circ e$. We need to prove $f=g$. Let $m: M \longmapsto X$ be the equaliser of $f, g$, with $h=\operatorname{coker}(m)$ :

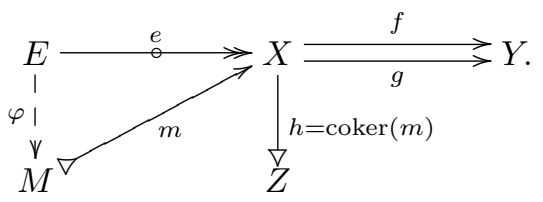

This $e$ factors through the equaliser $m$, as indicated, since $f \circ e=g \circ e$. Then $h \circ e=h \circ m \circ \varphi=0 \circ \varphi=0$. Hence $h=0$ because $e$ is a zero epi. But then $m=\operatorname{ker}(h)=\operatorname{ker}(0)=$ id, so that $f=g$.

3.4.7 Lemma In a dagger kernel category,

(a) $e$ is a zero epi if and only if $\operatorname{coker}(e)=0$;

(b) $\operatorname{coker}(f \circ e)=\operatorname{coker}(f)$ when $e$ is a zero epi;

(c) a kernel which is zero epi is an isomorphism.

Needless to say, since a dagger category is self-dual, the dual statements of all results in this section until now are also true. For example, $\operatorname{ker}(m \circ f)=\operatorname{ker}(f)$ for a zero mono $m$.

3.4.8 Proposition In Rel there are proper inclusions

kernel $\subsetneq$ dagger mono $\subsetneq$ mono $\subsetneq$ zero mono. 
In PInj there are proper identities

$$
\text { kernel }=\text { dagger mono }=\text { mono }=\text { zero mono. }
$$

In Hilb and PHilb one has

$$
\text { kernel }=\text { dagger mono } \subsetneq \text { mono }=\text { zero mono. }
$$

PROOF We still need to produce a zero mono in Rel that is not mono, and prove that every zero mono in PHilb is a mono. For the first, consider the relation $R \subseteq\{0,1\} \times\{a, b\}$ given by $R=\{(0, a),(1, a)\}$. Its first leg is surjective, so $R$ is a zero mono by the dual of Lemma 3.4.7(a) and Example 3.2.23. But it is not a mono: there are two different relations $\{(*, 0)\},\{(*, 1)\} \subseteq\{*\} \times\{0,1\}$ with $R \circ\{(*, 0)\}=\{(*, a)\}=R \circ\{(*, 1)\}$.

For the second, let $m: Y \rightarrow Z$ be a zero mono, and let $f, g: X \rightrightarrows Y$ be arbitrary morphisms in PHilb. More precisely, let $m, f$ and $g$ be morphisms in Hilb representing the equivalence classes $[m],[f]$ and $[g]$, which are morphisms in PHilb. Suppose that $[m \circ f]=[m \circ g]$. Then $m \circ f \sim m \circ g$, say $m \circ f=z \cdot m \circ g$ for $z \in U(1)$. So $m \circ(f-z \cdot g)=0$, and $f-z \cdot g=0$ since $m$ is zero mono. Then $f=z \cdot g$ and hence $f \sim g$, i.e. $[f]=[g]$. Thus $m$ is mono.

The following concept was popularised by Peter Freyd and Max Kelly [89], but its origins can be traced back to Saunders Mac Lane [161] and John Isbell [121] (see also [23, Exercises 5.5] or [33, Section 5.5]).

3.4.9 Definition A factorisation system $(E, M)$ for a category $\mathbf{C}$ consists of two classes $E$ and $M$ of morphisms of $\mathbf{C}$ such that

- $E$ and $M$ both contain all isomorphisms of $\mathbf{C}$, and are closed under composition;

- every morphism $f$ of $\mathbf{C}$ can be factored as $f=i \circ e$ for some $i \in M$ and $e \in E$; and

- the factorisation is functorial, in the sense that for morphisms $u, v$ with $v \circ i \circ e=i^{\prime} \circ e^{\prime} \circ u$ for $i, i^{\prime} \in M$ and $e, e^{\prime} \in E$, there is a unique morphism $w$ making the following diagram commute:

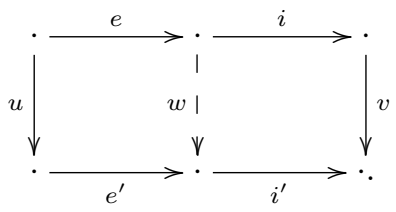


We will often denote $i \in M$ and $e \in E$ with $i \circ e=f$ by $i_{f}$ and $e_{f}$.

3.4.10 The requirement that a factorisation system $(E, M)$ is functorial is often formulated as the following equivalent diagonal fill-in property. For $e \in E, i \in M$ and arbitrary morphisms $f, g$ satisfying $i \circ f=g \circ e$, there is a unique $d$ such that $f=d \circ e$ and $g=i \circ d$.

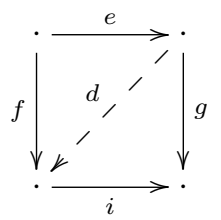

3.4.11 "Factorisation" can be taken more literally by viewing $M$ and $E$ as subcategories of $\mathbf{C}$ and saying $\mathbf{C}=M \circ E$ [187]. In any factorisation system, each of the classes $E$ and $M$ determines the other via 'orthogonality' [33, Proposition 5.5.3]. Let us call a factorisation system $(E, M)$ in a dagger category a dagger factorisation system when $M^{\dagger}=E$. A dagger factorisation system then resembles a square root, as $\mathbf{C}=E^{\dagger} \circ E$, or " $E=\sqrt{\mathbf{C}}$ ".

3.4.12 Example Any posetal category has a factorisation system where $E$ consists of all identity morphisms, and $M$ comprises all morphisms.

3.4.13 Example Regular categories, as discussed in 2.5.11, have a factorisation system consisting of monomorphisms and regular epis; see [169, Section 3.4] or [23, Exercise 5.5.4].

For example, the category Vect is regular, and hence it has a factorisation system of monos and regular epis. The regular epis in Vect are just the surjective morphisms [33, Example 4.3.10a], and the monos are just the injective ones.

The next few lemma's lead to a theorem stating that dagger kernel categories have a factorisation system consisting of zero epis and dagger kernels.

3.4.14 Any morphism $f: X \rightarrow Y$ in a dagger kernel category factors through $\operatorname{Im}(f)=\operatorname{ker}(\operatorname{coker}(f))$ via $i_{f}: \operatorname{Im}(f) \longmapsto \longrightarrow$, as follows. First take the kernel

$$
\operatorname{ker}\left(f^{\dagger}\right) \triangleright^{k} \longrightarrow Y \stackrel{f^{\dagger}}{\longrightarrow} X,
$$

and define $i_{f}$ as the dagger kernel of $k^{\dagger}$.

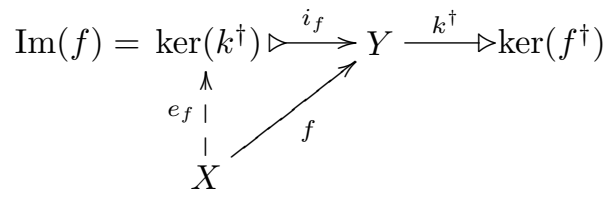


The map $e_{f}: X \rightarrow \operatorname{Im}(f)$ is obtained from the universal property of kernels, since $k^{\dagger} \circ f=\left(f^{\dagger} \circ k\right)^{\dagger}=0^{\dagger}=0$. Since $i_{f}$ is a dagger mono, this $e_{f}$ is determined as $e_{f}=\left(i_{f}\right)^{\dagger} \circ i_{f} \circ e_{f}=\left(i_{f}\right)^{\dagger} \circ f$.

The image of $f$ is therefore defined as kernel $\operatorname{ker}(\operatorname{coker}(f))$. Conversely, every kernel $m=\operatorname{ker}(f)$ arises as an image, since $\operatorname{ker}(\operatorname{coker}(m))=m$ by Lemma 3.4.1.

The maps arising as $e_{f}$ in (3.5) can be characterised as follows.

3.4.15 Lemma The morphisms in a dagger kernel category that arise of the form $e_{f}$, as in (3.5), are precisely the zero epis.

PRoOF We first show that $e_{f}$ is a zero epi. Assume that $h: \operatorname{ker}\left(k^{\dagger}\right) \rightarrow Z$ satisfies $h \circ e_{f}=0$. Since $e_{f}=\left(i_{f}\right)^{\dagger} \circ f$, we have

$$
f^{\dagger} \circ\left(i_{f} \circ h^{\dagger}\right)=\left(h \circ\left(i_{f}\right)^{\dagger} \circ f\right)^{\dagger}=\left(h \circ e_{f}\right)^{\dagger}=0^{\dagger}=0 .
$$

This means that $i_{f} \circ h^{\dagger}$ factors through the kernel of $f^{\dagger}$, say via $a: Z \rightarrow \operatorname{ker}\left(f^{\dagger}\right)$ with $k \circ a=i_{f} \circ h^{\dagger}$. Since $k$ is a dagger mono we now get

$$
a=k^{\dagger} \circ k \circ a=k^{\dagger} \circ i_{f} \circ h^{\dagger}=0 \circ h^{\dagger}=0 .
$$

But then $i_{f} \circ h^{\dagger}=k \circ a=k \circ 0=0=i_{f} \circ 0$, so that $h^{\dagger}=0$ because $i_{f}$ is mono. Thus $h=0$, as required.

Conversely, assume that $g: X \rightarrow Y$ is a zero epi. Then $\operatorname{coker}(g)=0$ by Lemma 3.4.7. By the same Lemma, $\operatorname{ker}\left((0 \rightarrow X)^{\dagger}\right)$ is the identity $\operatorname{id}_{X}$. By construction, this is the image of $g$, so that $e_{g}=g$.

3.4.16 Lemma In a dagger kernel category, any commuting square that is of the

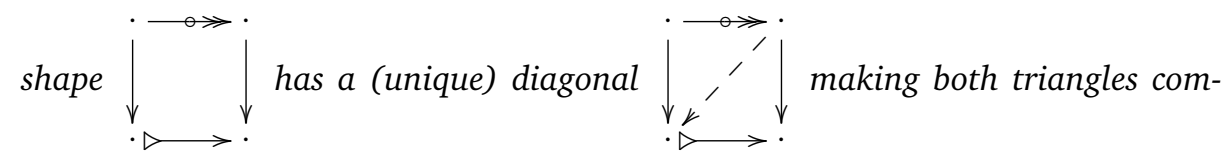
mute.

Proof Assume the zero epi $e: E \rightarrow Y$ and the kernel $k=\operatorname{ker}(h): K \triangleright \longrightarrow X$ satisfy $k \circ f=g \circ e$.

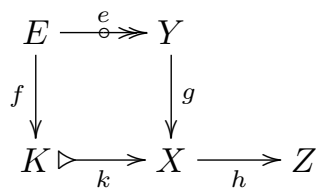

Then $h \circ g \circ e=h \circ k \circ f=0 \circ f=0$ and hence $h \circ g=0$, because $e$ is zero epi. This yields the required diagonal $d: Y \rightarrow K$ with $k \circ d=g$, because $k$ is the kernel of $h$. Using that $k$ is monic we get $d \circ e=f$. 
3.4.17 Theorem Dagger kernel categories have a factorisation system consisting of zero epis and (dagger) kernels.

As a corollary to the previous theorem, the following lemma brings more clarity to our running examples in Proposition 3.4.8. Compare also Lemma 3.4.6.

3.4.18 Lemma In any dagger kernel category: if zero epis are (ordinary) epis, then dagger monos are kernels.

Proof Suppose $m: M \longmapsto X$ is a dagger mono, with factorisation $m=i \circ e$ as in (3.5), where $i$ is a dagger kernel, and $e$ is a zero epi and hence an epi by assumption. We are done if we can show that $e$ is an isomorphism. Since $m=i \circ e$ and $i$ is dagger mono, we have $i^{\dagger} \circ m=i^{\dagger} \circ i \circ e=e$. Hence

$$
e^{\dagger} \circ e=\left(i^{\dagger} \circ m\right)^{\dagger} \circ e=m^{\dagger} \circ i \circ e=m^{\dagger} \circ m=\mathrm{id} .
$$

But then also $e \circ e^{\dagger}=\mathrm{id}$, because $e$ is epi and $e \circ e^{\dagger} \circ e=e$.

3.4.19 Suppose that $\mathbf{D}$ is a dagger equaliser category in which every dagger mono is a kernel, and coequalisers are stable under pullback. Then it is also a regular category (cf. 2.5.11), and the factorisation systems of Example 3.4.13 and Theorem 3.4.17 coincide by Lemma 3.4.6. It is tempting to call such categories dagger regular categories.

3.4.20 Theorem 3.4.17 shows that any dagger kernel category has a factorisation system consisting of zero epis and dagger kernels. Equivalently, it has a factorisation system of dagger cokernels and zero monos, by taking daggers. Both factorisation systems can be combined as follows. Any map $f: X \rightarrow Y$ in a dagger kernel category factors as $f=i_{f} \circ e_{f}$, where $i_{f}=\operatorname{ker}(\operatorname{coker}(f))$ is a kernel and $e_{f}$ is a zero epi. We can apply the same factorisation to $f^{\dagger}$. The dual of its image, $\left(i_{f^{\dagger}}\right)^{\dagger}=\operatorname{coker}(\operatorname{ker}(f)): X \longrightarrow \operatorname{Im}\left(f^{\dagger}\right)$, is commonly called the coimage of $f$. It is a dagger cokernel by construction. Thus we have
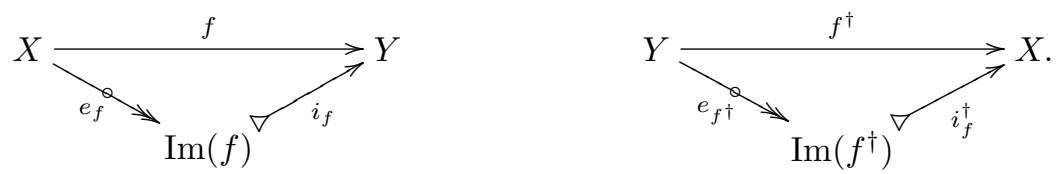
By combining these factorisations we get two mediating maps $m$ by diagonal fill-in:
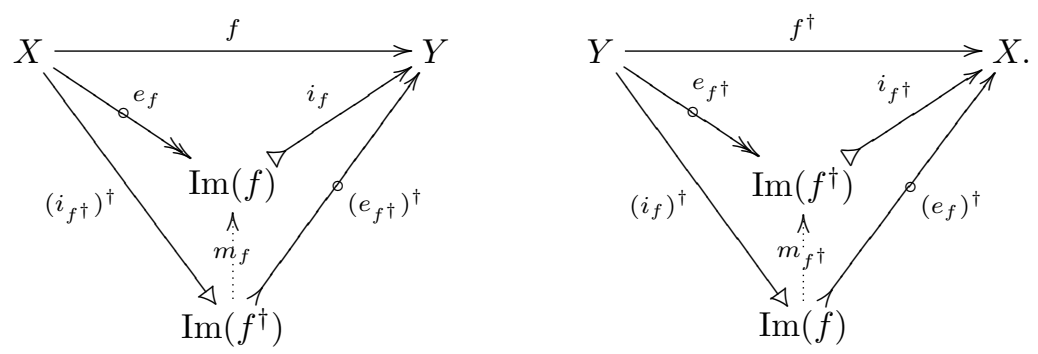

We claim that $\left(m_{f}\right)^{\dagger}=m_{f^{\dagger}}$. This easily follows from the fact that $\left(i_{f^{\dagger}}\right)^{\dagger}$ is epi:

$$
\left(m_{f^{\dagger}}\right)^{\dagger} \circ\left(i_{f^{\dagger}}\right)^{\dagger}=\left(i_{f^{\dagger}} \circ m_{f^{\dagger}}\right)^{\dagger}=\left(e_{f}\right)^{\dagger \dagger}=e_{f}=m_{f} \circ\left(i_{f^{\dagger}}\right)^{\dagger} .
$$

Moreover, $m_{f}$ is both a zero epi and a zero mono. As a result we can factorise each map $f: X \rightarrow Y$ as

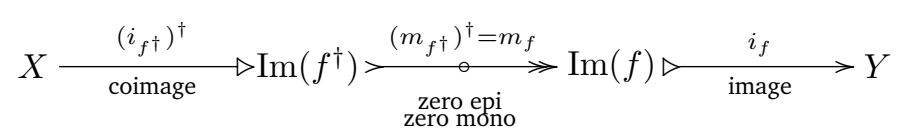

This coimage may also be reversed, so that a morphism can also be understood as a pair of kernels with a zero mono/epi between them:

$$
X \longleftarrow i_{f^{\dagger}} \triangleleft \operatorname{Im}\left(f^{\dagger}\right) \succ \longrightarrow \operatorname{Im}(f) \longmapsto i_{f} \quad \longrightarrow Y .
$$

We briefly describe the factorisation (3.6) in a few examples. These show that the two outer kernel maps perform some "bookkeeping" to adjust the types; the real action takes place in the middle. This can be thought of as a generalisation of polar decomposition in Hilbert space theory [104, Problem 105].

3.4.21 Example Any morphism $\left(X \stackrel{r_{1}}{\longleftarrow} R \stackrel{r_{2}}{\longrightarrow} Y\right.$ ) in Rel factors through the (set-theoretic) images $X^{\prime} \longmapsto X$ of $r_{1}$ and $Y^{\prime} \hookleftarrow Y$ of $r_{2}$. In diagrammatic order (with notation $f ; g=g \circ f$ ):

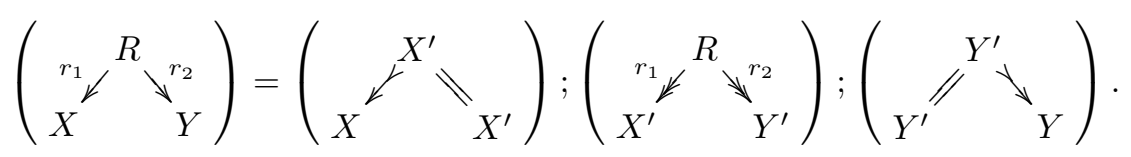

In the spirit of 3.4.11, the above three-way factorisation system can be refined by splitting the middle class, of zero monos that are zero epic, in two parts 
to arrive at a (two-way) factorisation system that is compatible with the dagger of Rel, as follows. Any morphism in Rel factors as the adjoint of (the graph of) a function followed by (the graph of) a function:

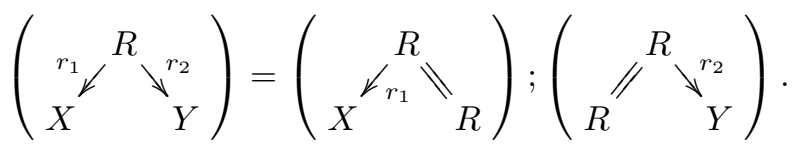

3.4.22 Example In PInj, the middle part $m$ in 3.6 is the identity:

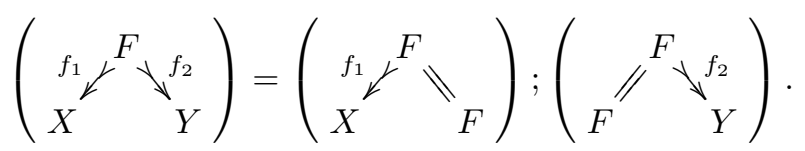

3.4.23 Example A morphism $f: X \rightarrow Y$ in Hilb factors as $f=i \circ m \circ e$. The third part $i: I \rightarrow Y$ is given by $i(y)=y$, where the image $I$ is the closure $\overline{\{f(x) \mid x \in X\}}$. The first part $e: X \rightarrow E$ is given by orthogonal projection on the closure $E=\overline{\left\{f^{\dagger}(y) \mid y \in Y\right\}}$; explicitly, $e(x)$ is the unique $x^{\prime}$ such that $x=x^{\prime}+x^{\prime \prime}$ with $x^{\prime} \in E$ and $\left\langle x^{\prime \prime} \mid z\right\rangle=0$ for all $z \in E$ (see Example 4.2.4). Using the fact that the adjoint $e^{\dagger}: E \rightarrow X$ is given by $e^{\dagger}(x)=x$, we deduce that the middle part $m: E \rightarrow I$ is determined by $m(x)=(i \circ m)(x)=\left(f \circ e^{\dagger}\right)(x)=f(x)$. Explicitly,

$$
(X \stackrel{f}{\longrightarrow} Y)=(X \stackrel{e}{\longrightarrow} E) ;(E \stackrel{m}{\longrightarrow} I) ;(I \stackrel{i}{\longrightarrow} Y) .
$$

This description descends to PHilb.

3.4.24 Definition A morphism $f$ in a dagger category is called a partial isometry when it factors as $f=i \circ e$ for a dagger mono $i$ and a dagger epi $e$.

3.4.25 In the category Hilb, the previous definition coincides with the usual notion. Every morphism in PInj is a partial isometry, and the functor $\ell^{2}: \mathbf{P I n j} \rightarrow$ Hilb preserves partial isometries.

The composition of partial isometries need not be a partial isometry again. For example, consider the partial isometries $\kappa: \mathbb{C} \rightarrow \mathbb{C}^{2}$ and $\frac{1}{2} \sqrt{2} \nabla: \mathbb{C}^{2} \rightarrow \mathbb{C}$ in Hilb. Their composite is given by $x \mapsto \frac{1}{2} \sqrt{2} x$. This is not a partial isometry anymore, since it factors as $\mathbb{C} \stackrel{1 / \sqrt{2}}{\longrightarrow} \mathbb{C} \triangleright \stackrel{\text { id }}{\longrightarrow} \mathbb{C}$.

3.4.26 Lemma If $f$ is a partial isometry in a dagger category, then $f^{\dagger} \circ f$ and $f \circ f^{\dagger}$ are projections. In dagger equaliser categories the converse also holds. Moreover, if one of $f \circ f^{\dagger}$ and $f^{\dagger} \circ f$ is a projection, so is the other. 
Proof Suppose that $f$ is a partial isometry, say $f=i$ o $e$ for a dagger mono $i$ and a dagger epi $e$. Then $f^{\dagger} \circ f \circ f^{\dagger} \circ f=e^{\dagger} \circ i^{\dagger} \circ i \circ e \circ e^{\dagger} \circ i^{\dagger} \circ i \circ e=e^{\dagger} \circ i^{\dagger} \circ i \circ e=f^{\dagger} \circ f$, and $\left(f^{\dagger} \circ f\right)^{\dagger}=f^{\dagger} \circ f$, so $f^{\dagger} \circ f$ is a projection. Likewise, $f \circ f^{\dagger}$ is a projection.

Conversely, suppose that $f^{\dagger} \circ f$ is a projection. Factorise $f=i \circ e$ for a zero epi $e$ and a dagger kernel $i$. Since $f^{\dagger} \circ f \circ f^{\dagger} f=f^{\dagger} \circ f$, we have

$$
e^{\dagger} \circ e=e^{\dagger} \circ i^{\dagger} \circ i \circ e=e^{\dagger} \circ i^{\dagger} \circ i \circ e \circ e^{\dagger} \circ i^{\dagger} \circ i \circ e=e^{\dagger} \circ e \circ e^{\dagger} \circ e .
$$

Because $e$ is epi by Lemma 3.4.6 it follows that $e^{\dagger}=e^{\dagger} \circ e \circ e^{\dagger}$. And because $e^{\dagger}$ is mono, one has id $=e \circ e^{\dagger}$. So $e$ is a dagger epi, and hence $f$ is a partial isometry.

\subsection{Hilbert modules}

This section develops another example of a dagger kernel category, namely that of Hilbert modules over an involutive rig, and studies its categorical properties. Analogously to Section 2.5, one could say that this category is the prime example of a dagger monoidal category with dagger biproducts, for this section shows that the latter always embed into a category of Hilbert modules.

3.5.1 A (commutative) involutive rig is a (commutative) rig $R$ that is equipped with a function $\ddagger: R \rightarrow R$ satisfying

$$
\begin{aligned}
r^{\ddagger \ddagger} & =r, \\
(r+s)^{\ddagger} & =r^{\ddagger}+s^{\ddagger}, \\
(r \cdot s)^{\ddagger} & =s^{\ddagger} \cdot r^{\ddagger}, \\
0^{\ddagger} & =0 .
\end{aligned}
$$

An element $r$ of an involutive rig is called positive, denoted $r \geq 0$, when it is of the form $r=s^{\ddagger} \cdot s$. The set of all positive elements of an involutive rig $R$ is denoted by $R^{+}$.

3.5.2 Let $R$ be an involutive rig. For every left- $R$-module $X$, there is also a right- $R$-module $X^{\ddagger}$, whose carrier set and addition are the same as before, but whose scalar multiplication $x \cdot r$ is defined in terms of the scalar multiplication of $X$ by $r^{\ddagger} \cdot x$.

A morphism $f: X \rightarrow Y$ of ${ }_{R} \operatorname{Mod}_{S}$ induces a morphism $f^{\ddagger}: X^{\ddagger} \rightarrow Y^{\ddagger}$ of ${ }_{S} \operatorname{Mod}_{R}$, by $f^{\ddagger}(m)=f(m)$. Thus an involution $\ddagger$ on a commutative rig $R$ induces an involutive functor $\ddagger: \operatorname{Mod}_{R} \rightarrow \operatorname{Mod}_{R}$.

Now, just as pre-Hilbert spaces are vector spaces over an involutive field carrying an inner product ( $c f$. Example 2.1.2), we can consider modules over 
involutive rigs with an inner product in general. The following definition is a novel extension of Hilbert modules over less general notions than involutive rigs.

3.5.3 Definition Let $R$ and $S$ be involutive rigs. A left- $R$-right- $S$-module $X$ is called a Hilbert left-R-right-S-module, or simply Hilbert module, when it is equipped with a morphism $\left\langle_{-} \mid \_\right\rangle: X^{\ddagger} \otimes X \rightarrow S$ of ${ }_{S} \operatorname{Mod}_{S}$, satisfying

- $\langle x \mid y\rangle=\langle y \mid x\rangle^{\ddagger}$;

- $\langle x \mid x\rangle \geq 0$;

- $\left\langle x \mid \_\right\rangle=\left\langle\left. y\right|_{\_}\right\rangle \Rightarrow x=y$.

The Hilbert module is called strict if furthermore

- $\langle x \mid x\rangle=0 \Rightarrow x=0$.

3.5.4 Example A commutative involutive rig $R$ is itself a Hilbert $R$-module by $\langle r \mid s\rangle_{R}=r^{\ddagger} \cdot s$. Recall that a rig $R$ is multiplicatively cancellative when $r \cdot s=r \cdot t$ and $r \neq 0$ imply $s=t$ [94]. In that case, $R$ is a strict Hilbert $R$-module.

3.5.5 Example Hilbert $C^{*}$-modules [152] are examples of Hilbert modules over a $C^{*}$-algebra. These special kinds of rings will be introduced in Chapter 5 , where they play an important role. Such Hilbert modules are automatically strict, since the base rig is a ring, in which subtraction is available.

3.5.6 Example Hilbert modules over involutive rigs also generalise Hilbert modules over involutive quantales [175]. A quantale is basically a rig in which addition can take an infinite number of arguments. More precisely, a quantale is a monoid in the category of complete lattices.

The following choice of morphisms is also the standard choice of morphisms between Hilbert modules over $\mathrm{C}^{*}$-algebras and Hilbert modules over quantales.

3.5.7 Definition A function $f: X \rightarrow Y$ between Hilbert $R$-modules is adjointable when there is a function $f^{\dagger}: Y \rightarrow X$ such that $\left\langle f^{\ddagger}(x) \mid y\right\rangle_{Y}=\left\langle x \mid f^{\dagger}(y)\right\rangle_{X}$ for all $x \in X^{\ddagger}$ and $y \in Y$. Adjointable functions are automatically module homomorphisms.

We will mostly be interested in commutative involutive rigs $R$. Hilbert $R$ modules and adjointable maps organise themselves in a category $\mathbf{H M o d}_{R}$. We denote by $\mathbf{s H M o d}_{R}$ the full subcategory of strict Hilbert $R$-modules. 
3.5.8 There is another analogy for this choice of morphisms. If we write $X^{*}$ for the dual $R$-module $\operatorname{Mod}(X, R)$ of $X$, Definition 3.5.3 resembles that of a 'diagonal' object of the Chu construction [20] on $\operatorname{Mod}_{R}$. The Chu construction provides a 'generalised topology', like an inner product provides a vector space provides with a metric and hence a topology [22].

3.5.9 The adjoint $f^{\dagger}$ is unique since the power transpose of the inner product is a monomorphism by definition. However, it does not necessarily exist, except in special situations like (Cauchy-complete) Hilbert spaces $(R=\mathbb{C}$ or $R=\mathbb{R})$ ) and bounded semilattices ( $R=\mathbb{B}$, see [175]).

The choice of morphisms ensures that $\mathbf{H M o d}_{R}$ and $\mathbf{s H M o d}{ }_{R}$ are dagger categories. We now study some of their properties. The following lemma could be read as a generalisation of the Riesz-Fischer theorem [181, Theorem III.1].

3.5.10 Proposition The category $\operatorname{HMod}_{R}$ is enriched over $\operatorname{Mod}_{R}$, and

$$
\operatorname{HMod}_{R}(R, X)=\operatorname{Mod}_{R}(R, X) \cong X,
$$

where we suppress the forgetful functor $\mathbf{H M o d}_{R} \rightarrow \operatorname{Mod}_{R}$.

Proof For $X, Y \in \operatorname{HMod}_{R}$, the zero map $X \rightarrow Y$ in $\operatorname{Mod}_{R}$ is adjointable, and hence a morphism in $\operatorname{HMod}_{R}$; its adjoint is the zero map $Y \rightarrow X$. If $f, g: X \rightrightarrows Y$ are adjointable, then so is $f+g$, as its adjoint is $f^{\dagger}+g^{\dagger}$. If $r \in R$ and $f: X \rightarrow Y$ is adjointable, then so is $r \cdot f$, as its adjoint is $r^{\ddagger} \cdot f^{\dagger}$ :

$$
\langle r \cdot f(x) \mid y\rangle_{Y}=r^{\ddagger} \cdot\langle f(x) \mid y\rangle_{Y}=r^{\ddagger} \cdot\left\langle x \mid f^{\dagger}(y)\right\rangle_{X}=\left\langle x \mid r^{\ddagger} \cdot f^{\dagger}(y)\right\rangle_{X} .
$$

Since composition is bilinear, $\operatorname{HMod}_{R}$ is enriched over $\operatorname{Mod}_{R}$.

Suppose $X \in \operatorname{HMod}_{R}$, and $f: R \rightarrow X$ is a morphism in $\operatorname{Mod}_{R}$. Define a morphism $f^{\dagger}: X \rightarrow R$ in $\operatorname{Mod}_{R}$ by $f^{\dagger}=\langle f(1) \mid-\rangle_{X}$. Then

$$
\langle f(r) \mid x\rangle_{X}=\langle r \cdot f(1) \mid x\rangle_{X}=r^{\ddagger} \cdot\langle f(1) \mid x\rangle_{X}=r^{\ddagger} \cdot f^{\dagger}(x)=\left\langle r \mid f^{\dagger}(x)\right\rangle_{R} .
$$

Hence $f \in \operatorname{HMod}_{R}(R, X)$. Obviously $\operatorname{HMod}_{R}(R, X) \subseteq \operatorname{Mod}_{R}(R, X)$. The fact that $R$ is a generator for $\operatorname{Mod}_{R}$ proves the last claim $\operatorname{Mod}_{R}(R, X) \cong X$.

3.5.11 Notice from the proof of the above proposition that the inner product of $X$ can be reconstructed from $\operatorname{HMod}_{R}(R, X)$. Indeed, if we temporarily define $\underline{x}: R \rightarrow X$ by $1 \mapsto x$ for $x \in X$, then we can use the adjoint by

$$
\langle x \mid y\rangle_{X}=\langle\underline{x}(1) \mid y\rangle_{X}=\left\langle 1 \mid \underline{x}^{\dagger}(y)\right\rangle_{S}=\underline{x}^{\dagger}(y)=\underline{x}^{\dagger} \circ \underline{y}(1) .
$$

We can go further by providing $\operatorname{HMod}_{R}(R, X)$ itself with the structure of a 
Hilbert $R$-module: for $f, g \in \operatorname{HMod}_{R}(R, X)$, put $\langle f \mid g\rangle_{\mathbf{H M o d}_{R}(R, X)}=f^{\dagger} \circ g(1)$. Then the above proposition can be strengthened as follows.

3.5.12 Proposition For any $X \in \mathbf{H M o d}_{R}$ there exists a dagger isomorphism $X \cong \operatorname{HMod}_{R}(R, X)$.

Proof Let $f: X \rightarrow \operatorname{HMod}_{R}(R, X)$ and $g: \operatorname{HMod}_{R}(R, X) \rightarrow X$ be the $R$ module homomorphisms determined by $f(x)=x \cdot\left(\_\right)$and $g(\varphi)=\varphi(1)$. Then $f \circ g=\mathrm{id}$ and $g \circ f=\mathrm{id}$, and furthermore $f^{\dagger}=g$ :

$$
\begin{aligned}
\langle x \mid g(\varphi)\rangle_{X} & =\langle x \mid \varphi(1)\rangle_{X}=\left(x \cdot\left({ }_{-}\right)\right)^{\dagger} \circ \varphi(1) \\
& =\left\langle x \cdot\left(\_\right) \mid \varphi\right\rangle_{\operatorname{HMod}_{R}(R, X)}=\langle f(x) \mid \varphi\rangle_{\operatorname{HMod}_{R}(R, X)}
\end{aligned}
$$

Recall that (a subset of) a rig is zerosumfree when $r+s=0$ implies $r=s=0$ for all elements $r$ and $s$ in it [94]. For example, $\mathbb{B}, \mathbb{Z}^{+}=\mathbb{N}, \mathbb{Q}^{+}$and $\mathbb{C}^{+}=\mathbb{R}^{+}$ are zerosumfree, but no ring is. Hence the following proposition for example applies to $\mathbb{N}, \mathbb{Z}, \mathbb{Q}, \mathbb{R}$ and $\mathbb{C}$.

3.5.13 Proposition The category $\operatorname{HMod}_{R}$ has finite dagger biproducts. When $R^{+}$is zerosumfree, $\mathbf{s H M o d}_{R}$ has finite dagger biproducts.

Proof Let $H_{1}, H_{2} \in \mathbf{H M o d}_{R}$ be given. Consider the $R$-module $H=H_{1} \oplus H_{2}$ from 2.5.3. Equip it with the inner product

$$
\left\langle h \mid h^{\prime}\right\rangle_{H}=\left\langle\pi_{1}(h) \mid \pi_{1}\left(h^{\prime}\right)\right\rangle_{H_{1}}+\left\langle\pi_{2}(h) \mid \pi_{2}\left(h^{\prime}\right)\right\rangle_{H_{2}} .
$$

Suppose that $\left\langle h \mid{ }_{-}\right\rangle_{H}=\left\langle\left. h^{\prime}\right|_{-}\right\rangle_{H}$. For every $i \in\{1,2\}$ and $h^{\prime \prime} \in H_{i}$, one then has

$$
\left\langle\pi_{i}(h) \mid h^{\prime \prime}\right\rangle_{H_{i}}=\left\langle h \mid \kappa_{i}\left(h^{\prime \prime}\right)\right\rangle_{H}=\left\langle h^{\prime} \mid \kappa_{i}\left(h^{\prime \prime}\right)\right\rangle_{H}=\left\langle\pi_{i}\left(h^{\prime}\right) \mid h^{\prime \prime}\right\rangle_{H_{i}},
$$

whence $\pi_{i}(h)=\pi_{i}\left(h^{\prime}\right)$, and so $h=h^{\prime}$. Thus $H$ is a Hilbert $R$-module. The maps $\kappa_{i}$ are morphisms of $\operatorname{HMod}_{R}$, as their adjoints are given by $\pi_{i}: H \rightarrow H_{i}$ :

$$
\left\langle h \mid \kappa_{i}\left(h^{\prime}\right)\right\rangle_{H}=\left\langle\pi_{1}(h) \mid \pi_{1} \kappa_{i}\left(h^{\prime}\right)\right\rangle_{H_{1}}+\left\langle\pi_{2}(h) \mid \pi_{2} \kappa_{i}\left(h^{\prime}\right)\right\rangle_{H_{2}}=\left\langle\pi_{i}(h) \mid h^{\prime}\right\rangle_{H_{i}} .
$$

For $\mathbf{s H M o d}_{R}$ we need to verify that $H$ is strict when $H_{1}$ and $H_{2}$ are. Suppose $\langle h \mid h\rangle_{H}=0$. Then $\left\langle\pi_{1}(h) \mid \pi_{1}(h)\right\rangle_{H_{1}}+\left\langle\pi_{2}(h) \mid \pi_{2}(h)\right\rangle_{H_{2}}=0$. Since $R^{+}$is zerosumfree, we have $\left\langle\pi_{i}(h) \mid \pi_{i}(h)\right\rangle_{H_{i}}=0$ for $i=1,2$. Hence $\pi_{i}(h)=0$, because $H_{i}$ is strict. Thus $h=0$, and $H$ is indeed strict.

3.5.14 Proposition The category $\mathbf{H M o d}_{R}$ is symmetric dagger monoidal. When $R$ is multiplicatively cancellative, $\mathbf{s H M o d}_{R}$ is symmetric dagger monoidal. 
Proof Let $H, K$ be Hilbert $R$-modules; then $H \otimes K$ is again an $R$-module. Define an equivalence relation $\sim$ on $H \otimes K$ by setting

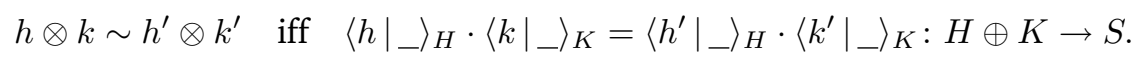

This is a congruence (see [94]), so $H \otimes_{H} K=H \otimes K / \sim$ is again an $R$-module. Defining an inner product on it by

$$
\left\langle[h \otimes k]_{\sim} \mid\left[h^{\prime} \otimes k^{\prime}\right]_{\sim}\right\rangle_{H \otimes H}=\left\langle h \mid h^{\prime}\right\rangle_{H} \cdot\left\langle k \mid k^{\prime}\right\rangle_{K}
$$

makes $H \otimes_{H} K$ into a Hilbert module.

Now let $f: H \rightarrow H^{\prime}$ and $g: K \rightarrow K^{\prime}$ be morphisms of $\operatorname{HMod}_{R}$. Define a morphism $f \otimes_{H} g: H \otimes_{H} K \rightarrow H^{\prime} \otimes_{H} K^{\prime}$ by $\left(f \otimes_{H} g\right)\left([h \otimes k]_{\sim}\right)=[f(h) \otimes g(k)]_{\sim}$. This is a well-defined function, for if $h \otimes k \sim h^{\prime} \otimes k^{\prime}$, then

$$
\begin{aligned}
\left\langle\left. f(h)\right|_{-}\right\rangle_{H^{\prime}} \cdot\left\langle g(k) \mid{ }_{-}\right\rangle_{K^{\prime}} & =\left\langle h \mid f^{\dagger}\left(\_\right)\right\rangle_{H} \cdot\left\langle k \mid g^{\dagger}\left(\_\right)\right\rangle_{K} \\
& =\left\langle h^{\prime} \mid f^{\dagger}\left(\_\right)\right\rangle_{H} \cdot\left\langle k^{\prime} \mid g^{\dagger}\left(\_\right)\right\rangle_{K} \\
& =\left\langle\left. f\left(h^{\prime}\right)\right|_{-}\right\rangle_{H^{\prime}} \cdot\left\langle g\left(k^{\prime}\right) \mid{ }_{-}\right\rangle_{K^{\prime}},
\end{aligned}
$$

and hence $\left(f \otimes_{H} g\right)(h \otimes k) \sim\left(f \otimes_{H} g\right)\left(h^{\prime} \otimes k^{\prime}\right)$. Moreover, it is adjointable, and hence a morphism of $\mathbf{H M o d}_{R}$ :

$$
\begin{aligned}
\left\langle\left(f \otimes_{H} g\right)(h \otimes k) \mid\left(h^{\prime} \otimes k^{\prime}\right)\right\rangle_{H^{\prime} \otimes_{H} K^{\prime}} & =\left\langle f(h) \otimes g(k) \mid h^{\prime} \otimes k^{\prime}\right\rangle_{H^{\prime} \otimes_{H} K^{\prime}} \\
& =\left\langle f(h) \mid h^{\prime}\right\rangle_{H^{\prime}} \cdot\left\langle g(k) \mid k^{\prime}\right\rangle_{K^{\prime}} \\
& =\left\langle h \mid f^{\dagger}\left(h^{\prime}\right)\right\rangle_{H} \cdot\left\langle k \mid g\left(k^{\prime}\right)\right\rangle_{K} \\
& =\left\langle h \otimes k \mid f^{\dagger}\left(h^{\prime}\right) \otimes g^{\dagger}\left(k^{\prime}\right)\right\rangle_{H \otimes_{H} K} \\
& =\left\langle h \otimes k \mid\left(f^{\dagger} \otimes g^{\dagger}\right)\left(h^{\prime} \otimes k^{\prime}\right)\right\rangle_{H \otimes_{H} K}
\end{aligned}
$$

In the same way, one shows that the coherence isomorphisms $\alpha, \lambda, \rho$ and $\gamma$ of the tensor product in $\operatorname{Mod}_{R}$ respect $\sim$, and descend to dagger isomorphisms in $\operatorname{HMod}_{R}$. For example:

$$
\begin{aligned}
\left\langle\lambda(r \otimes h) \mid h^{\prime}\right\rangle_{H} & =\left\langle r \cdot h \mid h^{\prime}\right\rangle_{H} \\
& =r^{\ddagger}\left\langle h \mid h^{\prime}\right\rangle_{H} \\
& =\langle r \mid 1\rangle_{R} \cdot\left\langle h \mid h^{\prime}\right\rangle_{H} \\
& =\left\langle r \otimes h \mid 1 \otimes h^{\prime}\right\rangle_{R \otimes_{H} H} \\
& =\left\langle r \otimes h \mid \lambda^{-1}\left(h^{\prime}\right)\right\rangle_{R \otimes_{H} H},
\end{aligned}
$$

so $\lambda^{\dagger}=\lambda^{-1}$. A routine check shows that $\left(\otimes_{H}, R\right)$ makes HMod $_{R}$ into a symmetric monoidal category. 
Finally, let us verify that these tensor products descend to $\operatorname{sHMod}_{R}$ when $R$ is multiplicatively cancellative. Suppose that

$$
0=\left\langle[h \otimes k]_{\sim} \mid[h \otimes k]_{\sim}\right\rangle_{H \otimes_{H} K}=\langle h \mid h\rangle_{H} \cdot\langle k \mid k\rangle_{K} .
$$

Then since $R$ is multiplicatively cancellative, either $\langle h \mid h\rangle_{H}=0$ or $\langle k \mid k\rangle_{H}=0$. Since $H$ and $K$ are assumed strict, this means that either $h=0$ or $k=0$. In both cases we conclude $[h \otimes k]_{\sim}=0$, so that $H \otimes_{H} K$ is indeed strict.

Theorem 2.5 .10 shows that categories with finite biproducts embed into some $\operatorname{Mod}_{R}$. We end this section by showing that in the presence of a dagger this embedding factors through $\operatorname{HMod}_{R}$. We start with an extension of Proposition 2.4.3.

3.5.15 Lemma Let $\mathbf{D}$ be a dagger symmetric monoidal category with finite dagger biproducts. Then $R=\mathbf{D}(I, I)$ is a commutative involutive rig.

Proof By Proposition 2.4.3, $R$ is a commutative rig. The dagger provides an involution on $R$. One easily verifies that this satisfies the requirements of 3.5.1. $\square$

3.5.16 Lemma Let $\mathbf{D}$ be a dagger symmetric monoidal category with finite dagger biproducts. There is a dagger functor $\mathbf{D}\left(I, \_\right): \mathbf{D} \rightarrow \mathbf{H M o d}_{R}$ for $R=\mathbf{D}(I, I)$.

Proof We start by putting an $R$-valued inner product on $\mathbf{D}(I, X)$. Inspired by Proposition 3.5.12, we define $\left\langle_{-} \mid{ }_{-}\right\rangle: \mathbf{D}(I, X)^{\ddagger} \otimes \mathbf{D}(I, X) \rightarrow \mathbf{D}(I, I)$ by (linear extension of) $\langle x \mid y\rangle=x^{\dagger} \circ y$ for $x, y \in \mathbf{D}(I, X)$. The Yoneda lemma shows that its power transpose $x \mapsto x^{\dagger} \circ\left(_{-}\right)$is a monomorphism. Thus $\mathbf{D}(I, X)$ is a Hilbert left- $R$-module.

Moreover, the image of a morphism $f: X \rightarrow Y$ of $\mathbf{D}$ under $\mathbf{D}\left(I, \_\right)$is indeed a morphism of $\operatorname{sHMod}_{R}$, that is, it is adjointable, since

$$
\langle f \circ x \mid y\rangle_{\mathbf{D}(I, Y)}=(f \circ x)^{\dagger} \circ y=x^{\dagger} \circ f^{\dagger} \circ y=\left\langle x \mid f^{\dagger} \circ y\right\rangle_{\mathbf{D}(I, X)}
$$

for $x \in \mathbf{D}(I, X)$ and $y \in \mathbf{D}(I, Y)$. This also shows that $\mathbf{D}\left(I,{ }_{-}\right)$is a dagger functor.

The next sections will consider preservation properties of the functor in the previous lemma, but we can at once see that it preserves dagger kernels.

3.5.17 Lemma Let $\mathbf{D}$ be a dagger symmetric monoidal category with finite dagger biproducts. The functor $\mathbf{D}\left(I,{ }_{-}\right): \mathbf{D} \rightarrow \mathbf{H M o d}_{R}$ of the previous lemma preserves dagger kernels. 
Proof Suppose that $k=\operatorname{ker}(f): K \bowtie \longrightarrow X$ is a kernel of $f: X \rightarrow Y$ in $\mathbf{D}$. We have to show that $\mathbf{D}(I, k)=k \circ\left(_{-}\right): \mathbf{D}(I, K) \triangleright \mathbf{D}(I, X)$ is a kernel of $\mathbf{D}(I, f)=f \circ\left(_{\_}\right): \mathbf{D}(I, X) \rightarrow \mathbf{D}(I, Y)$ in $\mathbf{s H M o d}_{R}$. First of all, one indeed has $\mathbf{D}(I, f) \circ \mathbf{D}(I, k)=\mathbf{D}(I, f \circ k)=0$. Now suppose that $l: Z \rightarrow \mathbf{D}(I, X)$ also satisfies $\mathbf{D}(I, f) \circ l=0$. That is, for all $z \in Z$, we have $f \circ(l(z))=0$. Since $k$ is a kernel, for each $z \in Z$ there is a unique $m_{z}: I \rightarrow K$ with $l(z)=k \circ m_{z}$. Define a function $m: Z \rightarrow \mathbf{D}(I, K)$ by $m(z)=m_{z}$. This is a well-defined module morphism, since $l$ is; for example,

$$
k \circ m_{z+z^{\prime}}=l\left(z+z^{\prime}\right)=l(z)+l\left(z^{\prime}\right)=\left(k \circ m_{z}\right)+\left(k \circ m_{z^{\prime}}\right)=k \circ\left(m_{z}+m_{z^{\prime}}\right),
$$

so that $m\left(z+z^{\prime}\right)=m(z)+m\left(z^{\prime}\right)$ because $k$ is mono. In fact, $m$ is the unique module morphism satisfying $l=\mathbf{D}(I, k) \circ \mathrm{m}$. Since $k$ is a dagger mono, we have $m=\mathbf{D}\left(I, k^{\dagger}\right) \circ l$. So as a composition of adjointable module morphisms $m$ is a well-defined morphism of $\mathbf{s H M o d}_{R}$. Thus $\mathbf{D}(I, k)$ is indeed a kernel of $\mathbf{D}(I, f)$.

\subsection{Scalars revisited}

This section studies what consequences additional assumptions on a dagger symmetric monoidal category with dagger biproducts have for its scalars. We consider the following cases: dagger equalisers, the monoidal unit being a so-called simple object, and the monoidal unit being a generator. It turns out that under these circumstances the scalars form an involutive field, and even a subfield of the complex numbers. This is inspired by [211], and extends this paper in the following way: the single additional assumption that dagger monos are kernels guarantees the scalars to be a field, thus eliminating the need to freely add differences and quotients. This section also relates to [178, 215], although it uses no topology.

3.6.1 Definition An object $I$ of a category $\mathbf{D}$ is simple when $\operatorname{Sub}(I)=\{0, I\}$, and $\mathbf{D}(I, I)$ is at most of continuum cardinality.

Here $\operatorname{Sub}(I)$ denote the subobjects of $I$, i.e. equivalence classes of monomorphisms into $I$ - this will be recalled in some detail in 4.1.1. There are always at least two subobjects, namely $0: 0 \longmapsto I$ and id: $I \longmapsto I$. When there are no others (and the size condition is satisfied), $I$ is simple. For example, $\mathbb{C}$ is a simple object in Hilb. Intuitively, simple objects can be thought of as 1-dimensional. They can also be thought of as supercompact topological spaces [81].

3.6.2 Convention For the rest of this section, we fix a dagger symmetric monoidal category $\mathbf{D}$ with finite dagger biproducts, and denote its commutative in- 
volutive rig of scalars by $R=\mathbf{D}(I, I)$.

3.6.3 Lemma If $\mathbf{D}$ has dagger kernels, then the functor $\mathbf{D}\left(I,_{-}\right): \mathbf{D} \rightarrow \mathbf{H M o d}_{R}$ of Lemma 3.5.16 takes values in $\mathbf{s H M o d}_{R}$.

Proof [211, Lemma 2.11] We have to prove that $x^{\dagger} \circ x=0$ implies $x=0$, for every $x: I \rightarrow X$ in $\mathbf{D}$. If $x^{\dagger} \circ x=0$, then $x$ factors through $k=\operatorname{ker}\left(x^{\dagger}\right)$, say by $x=k \circ x^{\prime}$ :

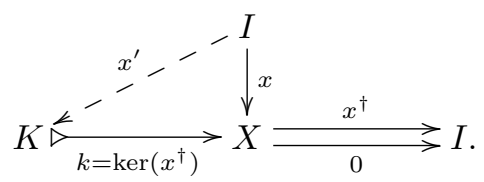

But since kernels are dagger monos,

$$
x=k \circ x^{\prime}=k \circ k^{\dagger} \circ k \circ x^{\prime}=k \circ \operatorname{coker}(x) \circ x=k \circ 0=0 .
$$

3.6.4 Corollary If $\mathbf{D}$ has dagger kernels, then $R^{+}$is zerosumfree.

PRoof Suppose that scalars $r, s: I \rightrightarrows I$ satisfy $r^{\dagger} \circ r+s^{\dagger} \circ s=0$. By definition,

$$
\begin{aligned}
0 & =r^{\dagger} \circ r+s^{\dagger} \circ s \\
& =\nabla \circ\left(r^{\dagger} \circ r \oplus s^{\dagger} \circ s\right) \circ \Delta \\
& =\nabla \circ\left(r^{\dagger} \oplus s^{\dagger}\right) \circ(r \oplus s) \circ \Delta \\
& =((r \oplus s) \circ \Delta)^{\dagger} \circ((r \oplus s) \circ \Delta) .
\end{aligned}
$$

Hence by the previous lemma $(r \oplus s) \circ \Delta=0$. That means $\langle r, s\rangle=(r \oplus s) \circ \Delta=$ $0=\langle 0,0\rangle$. Thus $r=s=0$.

3.6.5 Lemma If $\mathbf{D}$ has equalisers and $I$ is simple, then $R$ is multiplicatively cancellative.

PRoOF [211, 3.5] Suppose that $r \circ s=r \circ t$ and $r \neq 0$ for scalars $r, s, t \in R$. Then the equaliser $e=\mathrm{eq}(s, t) \in \operatorname{Sub}(I)$. Since $I$ is assumped simple, $e$ is either zero or isomorphic. We will show the impossibility of $e=0$, so that it must be isomorphic, whence $s=t$. Because $r \circ s=r \circ t$, there is a $\bar{r}$ such that $r=e \circ \bar{r}$.

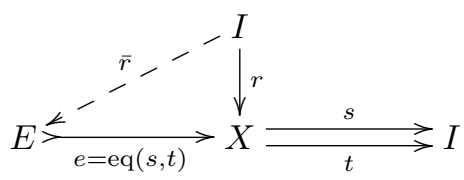

So if $e=0$, then $r=e \circ \bar{r}=0 \circ \bar{r}=0$, which is a contradiction. 
The previous lemma can be strengthened. An (involutive) division rig is a (involutive) rig in which every nonzero element has a multiplicative inverse. Commutative division rigs are also called semifields [94].

3.6.6 Lemma If $\mathbf{D}$ has dagger equalisers, every dagger mono is a kernel, and $I$ is simple, then $R$ is a division rig, wich multiplicative inverse $r^{-1}$ given by compositional inverse $r^{-1}$.

PROOF We will show that $R$ is a division rig by proving that any $r \in R$ is either zero or iso, using the factorisation of Theorem 3.4.17. Factorise $r$ as $r=i \circ e$ for a dagger mono $i: J \triangleright \longrightarrow I$ and a zero epi $e: I \longrightarrow 0$. Since $I$ is assumed simple, either $i$ is zero or $i$ is isomorphic. If $i=0$ then $r=0$. If $i$ is iso, then $r$ is zero epi. By Lemma 3.4.6, $r$ is epi, so $r^{\dagger}$ is mono. Again, either $r^{\dagger}=0$, in which case $r=0$, or $r^{\dagger}$ is isomorphic. In this last case $r$ is also isomorphic.

3.6.7 Convention Until now, we have allowed rigs $R$ with $0=1$. Sometimes this unique trivial rig is excluded from consideration by convention. For example, fields usually require $0 \neq 1$ by definition. In our case, the rig $R$ being trivial is equivalent to the category $\mathbf{D}$ being trivial, i.e. $\mathbf{D}$ being the one-morphism (and hence one-object) category. The results in the rest of this section assume $\mathbf{D}$ to be non-trivial. The main theorem, Theorem 3.7.18, holds regardlessly.

3.6.8 Lemma If $\mathbf{D}$ has dagger equalisers, every dagger mono is a kernel, and I is simple, then $R$ is an involutive field.

PRoof Applying [94, 4.34] to the previous lemma yields that $R$ is either zerosumfree, or a field. Assume, towards a contraction, that it is zerosumfree. Consider the kernel $k: K \rightarrow I \oplus I$ of the codiagonal $\nabla: I \oplus I \rightarrow I$. Defining $k_{i}=\pi_{i} \circ k: K \rightarrow I$, we have $k_{1}+k_{2}=0$. Hence also $k_{1} \circ k_{1}^{\dagger}+k_{2} \circ k_{1}^{\dagger}=0$, and so $k_{1} \circ k_{1}^{\dagger}=0$. But then $k_{1}=0$, and hence $k_{2}=0$. Thus $\operatorname{ker}(\nabla)=0$. But then, by Lemma 3.4.7(a) and Lemma 3.4.6, $\nabla$ is mono, whence $\kappa_{1}=\kappa_{2}$, which is a contradiction. Finally, the dagger on $\mathbf{D}$ provides an involution on the field $R$.

3.6.9 Note that the scalars of PHilb are $\mathbb{R}^{+}$, which is not a ring. Since $\mathbb{C}$ is a simple generator in PHilb, this entails that the category PHilb either does not have dagger equalisers, or does not have finite dagger biproducts. Likewise, the previous lemma shows that Rel does not have dagger equalisers.

3.6.10 Theorem If $\mathbf{D}$ has dagger equalisers, every dagger mono is a kernel, and $I$ is a simple generator, then $R$ is an involutive field of characteristic zero and at most continuum cardinality, with $R^{+}$zerosumfree. 
PRoOF We still have to prove that for all scalars $r: I \rightarrow I$ the property $r+$ $\cdots+r=0$ implies $r=0$, where the sum contains $n$ copies of $r$, for all $n \in$ $\{1,2,3, \ldots\}$. So suppose that $r+\cdots+r=0$. By definition, $r+\cdots+r=$ $\nabla^{n} \circ(r \oplus \cdots \oplus r) \circ \Delta^{n}=\nabla^{n} \circ \Delta^{n} \circ r$, where $\nabla^{n}=[\mathrm{id}]_{i=1}^{n}: \bigoplus_{i=1}^{n} I \rightarrow I$ and $\Delta^{n}=\langle\mathrm{id}\rangle_{i=1}^{n}: I \rightarrow \bigoplus_{i=1}^{n} I$ are the $n$-fold (co)diagonals. But $0 \neq \nabla^{n} \circ \Delta^{n}=$ $\left(\Delta^{n}\right)^{\dagger} \circ \Delta^{n}$ by Lemma 3.6.3. Since $R$ is a field, this means that $r=0$.

To prove that any field as in the previous theorem in fact embeds into the complex numbers, we cite two well-known results from algebra and model theory. Recall that a field is algebraically closed when every polynomial over it of degree at least 1 has a root.

3.6.11 Lemma [98. Theorem 4.4] Any field of characteristic zero and at most continuum cardinality can be embedded in an algebraically closed field of characteristic zero and continuum cardinality.

3.6.12 Lemma [47. Proposition 1.4.10] All algebraically closed fields of characteristic zero and continuum cardinality are isomorphic.

3.6.13 Theorem If $\mathbf{D}$ has dagger equalisers, every dagger mono is a kernel, and I is a simple generator, then there is a monomorphism $R \mapsto \mathbb{C}$ of fields.

3.6.14 The isomorphism of Lemma 3.6.12 is by no means canonical; there can be very many such isomorphisms. This is caused by the reliance of the proof of the above lemma on Zorn's lemma, or equivalently, on the axiom of choice. In particular, the embedding of fields of the previous theorem does not necessarily preserve the involution.

3.6.15 For (not necessarily symmetric) dagger monoidal categories, the scalars form only a skew field of characteristic zero and at most continuum cardinality. One might suspect that any such skew field embeds into the quaternions. However, this is not the case. First, there is no canonical definition stating that a skew field is algebraically closed [148]. Second, the technique of adding a continuum of transcendentals does not apply in this case [167]. Third, not all algebraically closed skew fields of the same cardinality are isomorphic [55].

3.6.16 There are size issues at play in the above theorem. Definition 3.6.1. of a simple object, requires that $R$ be of at most continuum cardinality, but Lemmas 3.6.11 and 3.6.12 hold for higher cardinalities as well. Moreover, the usual algebraic definition of simple object does not include the size requirement.

Thus, although the complex numbers are very familiar, we could also take Theorem 3.6.10 at face value, and drop the unappealing size requirement. Theorem 3.7 .8 below would then give an embedding into preHilb $_{R}$ for an involutive field $R$ instead of into preHilb $\mathbb{C}_{\mathbb{C}}$, but it would preserve the dagger on the nose. 
3.6.17 The above theorem is of interest to reconstruction programmes that try to the mathematical structure of quantum theory from simpler assumptions, for among the things to be reconstructed are the scalars [153, 177]. For example, [201] shows that if an orthomodular pre-Hilbert space is infinite dimensional, then the base field is either $\mathbb{R}$ or $\mathbb{C}$, and the space is a Hilbert space.

\subsection{Hilbert categories}

This section axiomatically defines (pre-)Hilbert categories as dagger categories that moreover enjoy the "dagger properties" that we studied in the previous sections. It finishes the chapter by proving analogues of the embedding theorem in Chapter 2 in the presence of a dagger. This justifies the names: (pre-)Hilbert categories embed into the category of (pre-)Hilbert spaces, preserving all pertinent structure.

3.7.1 Definition A category is called a pre-Hilbert category when:

- it has a dagger;

- it has finite dagger biproducts;

- it has (finite) dagger equalisers;

- every dagger mono is a kernel;

- it is symmetric dagger monoidal.

3.7.2 Lemma If $\mathbf{D}$ is an arbitrary pre-Hilbert category, then there is a functor $\mathbf{D}\left(I,{ }_{-}\right): \mathbf{D} \rightarrow \mathbf{s H M o d}_{R}$, where $R=\mathbf{D}(I, I)$. This functor:

(a) is faithful if and only if I is a generator;

(b) preserves the dagger;

(c) preserves dagger biproducts;

(d) preserves dagger equalisers, and hence all finite limits and colimits;

(e) is monoidal when I is simple;

Proof We already saw in Lemma 3.6.3 that the desired functor exists and that it satisfies (b) of that lemma. Also (a) has been demonstrated before in Theorem 2.5.10, so we start with (c). Notice that by Corollary 3.6.4, the category $\operatorname{sHMod}_{R}$ indeed has dagger biproducts, as described in Proposition 3.5.13. By definition of product in $\mathbf{D}$, we have $\mathbf{D}(I, X \oplus Y) \cong \mathbf{D}(I, X) \oplus \mathbf{D}(I, Y)$. 
We move to (d). It easy to see that $F=\mathbf{D}\left(I,{ }_{-}\right)$is an $\mathbf{A b}$-functor, that is, $(f+g) \circ\left(\_\right)=\left(f \circ{ }_{-}\right)+\left(g \circ{ }_{-}\right)$. Hence it follows from Lemma 3.5.17 that $F$ preserves (dagger) equalisers:

$$
F(\mathrm{eq}(f, g))=F(\operatorname{ker}(f-g))=\operatorname{ker}(F(f-g))=\operatorname{ker}(F f-F g)=\operatorname{eq}(F f, F g) .
$$

Since $F$ preserves equalisers and finite products, it preserves all finite limits. Because it also preserves the self-duality $\dagger$, also all finite colimits are preserved.

For (e), notice that by Lemma 3.6.6, the category $\mathbf{s H M o d}_{R}$ indeed has dagger tensor products, as described in Proposition 3.5.14. We must give a natural transformation $\varphi_{X, Y}: \mathbf{D}(I, X) \otimes \mathbf{D}(I, Y) \rightarrow \mathbf{D}(I, X \otimes Y)$ and a morphism $\psi: R \rightarrow \mathbf{D}(I, I)$. Since $R=\mathbf{D}(I, I)$, we can simply take $\psi=\mathrm{id}$. Define $\varphi$ by mapping $x \otimes y$ for $x: I \rightarrow X$ and $y: I \rightarrow Y$ to the composite

$$
I \stackrel{\cong}{\longrightarrow} I \otimes I \stackrel{x \otimes y}{\longrightarrow} X \otimes Y .
$$

It is easily seen that $\varphi$ and $\psi$ make the required coherence diagrams commute. $\square$

3.7.3 Just like in Theorem 2.5.10, the functor from the previous embedding is full when $I$ is a projective generator and every object of $\mathbf{D}$ is finitely projective. However, the technique used in Chapter 2 to embed a regular category fully into one with a projective generator that makes every object finitely projective does not apply here. There, moving from $\mathbf{C}$ to $[\mathbf{C}, \mathbf{c M o n}]$ provided a structurepreserving embedding of categories. But in the setting of dagger categories, there is no analogue of cMon. Moreover, whereas $\coprod_{X \in \mathbf{D}} \mathbf{D}\left(X,{ }_{-}\right)$would be the obvious candidate for a projective generator in $[\mathbf{D}, \mathbf{c M o n}]$, pre-Hilbert categories tend not to have infinite coproducts ( $c f$. 2.3.15 and 2.3.16.

3.7.4 If $\mathbf{H}$ is a pre-Hilbert category and $\mathbf{D}$ is a dagger category, then $[\mathbf{D}, \mathbf{H}]$ is again a pre-Hilbert category. But even if the monoidal unit of $\mathbf{H}$ is simple, the monoidal unit of $[\mathbf{D}, \mathbf{H}]$ no longer is for nontrivial $\mathbf{D}$. Hence an extension of the above embedding theorem to $I$ that are not necessarily simple requires reconstructing $\mathbf{D}$ from $\operatorname{Sub}(I)$. Compare footnote 1 on page 40 .

In a pre-Hilbert category $\mathbf{D}$ whose monoidal unit is a simple generator, the scalars embed into the complex field by Theorem 3.6.13. We will now construct a functor $\mathbf{H M o d}_{R} \rightarrow \operatorname{HMod}_{S}$ from a homomorphism $f: R \rightarrow S$ of involutive rigs, and apply this construction to $R \longmapsto \mathbb{C}$. Thus we will extend the previous theorem to a structure-preserving embedding $\mathbf{D} \rightarrow \mathbf{H M o d}_{\mathbb{C}}=\operatorname{preHilb}_{\text {. }}$

This technique is called extension of scalars, and is well known in the setting of modules (see e.g. [9, 10.8.8]). Let us first consider in some more detail the construction on modules over rigs. 
3.7.5 Let $R$ and $S$ be commutative rigs, and let $f: R \rightarrow S$ be a homomorphism of rigs. Then any $S$-module $X$ can be considered an $R$-module $X_{R}$ by defining scalar multiplication $r \cdot x$ in $X_{R}$ in terms of scalar multiplication in $X$ by $f(r) \cdot x$. In particular, we can regard $S$ as an $R$-module. Hence it makes sense to look at $S \otimes_{R} X$. Somewhat more precisely, we can view $S$ as a left- $S$-right- $R$-module, and $X$ as a left- $R$-module. Hence $S \otimes_{R} X$ becomes a (left-) $S$-module. This construction induces a functor $f^{*}: \operatorname{Mod}_{R} \rightarrow \operatorname{Mod}_{S}$, acting on morphisms $g$ as id $\otimes_{R} g$. It is easily seen to be strong monoidal and to preserve biproducts and kernels.

Moreover, the fact that any $S$-module can be seen as an $R$-module via $f$ immediately induces another functor $f_{*}: \operatorname{Mod}_{S} \rightarrow \operatorname{Mod}_{R}$. This one is called restriction of scalars along $f$. In fact, $f_{*}$ is right adjoint to $f^{*}[33,3.1 .6 \mathrm{e}]$.

Now let us change to the setting of Hilbert modules, where $R$ and $S$ are involutive rigs, and $f: R \rightarrow S$ is a morphism of involutive rigs. The next lemma shows that extension of scalars along $f$ lifts to a functor $f^{*}: \operatorname{sHMod}_{R} \rightarrow$ $\operatorname{sHMod}_{S}$ (under some conditions on $S$ and $f$ ). However, since we do not know how to fashion an $R$-valued inner product out of an $S$-valued one in general, it seems impossible to construct an adjoint functor $f_{*}: \mathbf{s H M o d}_{S} \rightarrow \mathbf{s H M o d}_{R}$.

3.7.6 Lemma Let $R$ be a commutative involutive rig, $S$ a multiplicatively cancellative commutative involutive ring, and $f: R \mapsto S$ a monomorphism of involutive rigs. There is a functor $f^{*}: \mathbf{s H M o d}_{R} \rightarrow \mathbf{s H M o d}_{S}$. This functor:

(a) is faithful;

(b) preserves the dagger;

(c) preserves dagger biproducts when both $R^{+}$and $S^{+}$are zerosumfree;

(d) is strong monoidal when $R$ is multiplicatively cancellative.

Proof Let $X$ be a strict Hilbert $R$-module. Defining the carrier of $f^{*} X$ to be $S \otimes_{R} X$ turns it into an $S$-module as before. Furnish it with

$$
\left\langle s \otimes x \mid s^{\prime} \otimes x^{\prime}\right\rangle_{f^{*} X}=s^{\ddagger} \cdot s^{\prime} \cdot f\left(\left\langle x \mid x^{\prime}\right\rangle_{X}\right) .
$$

Assume $0=\langle s \otimes x \mid s \otimes x\rangle_{f^{*} X}=s^{\ddagger} \cdot s \cdot f\left(\langle x \mid x\rangle_{X}\right)$. Since $S$ is multiplicatively cancellative, either $s=0$ or $f\left(\langle x \mid x\rangle_{X}\right)=0$. In the former case $s \otimes x=0$. In the latter case $\langle x \mid x\rangle_{X}=0$, since $f$ is injective, and because $X$ is strict this yields $x=0$, whence $s \otimes x=0$. Since $S$ is a ring, this implies that $f^{*} X$ is a strict Hilbert $S$-module. For if $\langle x \mid-\rangle_{f^{*} X}=\left\langle x^{\prime} \mid-\right\rangle_{f^{*} X}$ then $\left\langle x-x^{\prime} \mid-\right\rangle_{f^{*} X}=0$, so in particular $\left\langle x-x^{\prime} \mid x-x^{\prime}\right\rangle_{f^{*} X}=0$. Hence $x-x^{\prime}=0$ and $x=x^{\prime}$. 
Moreover, the image of a morphism $g: X \rightarrow X^{\prime}$ of $\operatorname{sHMod}_{R}$ under $f^{*}$ is a morphism of $\mathbf{s H M o d}_{S}$, as its adjoint is id $\otimes g^{\dagger}$ :

$$
\begin{aligned}
\left\langle(\mathrm{id} \otimes g)(s \otimes x) \mid s^{\prime} \otimes x^{\prime}\right\rangle_{f^{*} X^{\prime}} & =\left\langle s \otimes g(x) \mid s^{\prime} \otimes x^{\prime}\right\rangle_{f^{*} X^{\prime}} \\
& =s^{\ddagger} \cdot s^{\prime} \cdot f\left(\left\langle g(x) \mid x^{\prime}\right\rangle_{X^{\prime}}\right) \\
& =s^{\ddagger} \cdot s^{\prime} \cdot f\left(\left\langle x \mid g^{\dagger}\left(x^{\prime}\right)\right\rangle_{X}\right) \\
& =\left\langle s \otimes x \mid s^{\prime} \otimes g^{\dagger}\left(x^{\prime}\right)\right\rangle_{f^{*} X} \\
& =\left\langle s \otimes x \mid\left(\mathrm{id} \otimes g^{\dagger}\right)\left(s^{\prime} \otimes x^{\prime}\right)\right\rangle_{f^{*} X} .
\end{aligned}
$$

Obviously, $f^{*}$ is faithful, and preserves the dagger, proving (a) and (b). For (c): if dagger biproducts are available, then $f^{*}$ preserves them, since biproducts distribute over tensor products. Finally, we show (d). If tensor products are available, showing that $f^{*}$ preserves them comes down to giving an isomorphism $S \rightarrow S \otimes_{R} R$ and a natural isomorphism $\left(S \otimes_{R} X\right) \otimes_{S}\left(S \otimes_{R} Y\right) \rightarrow S \otimes_{R}\left(X \otimes_{R} Y\right)$. The obvious candidates for these satisfy the coherence diagrams, making $f^{*}$ strong monoidal.

3.7.7 The extension of scalars functor $f^{*}$ of the above lemma is full if and only if $f$ is a regular epimorphism, i.e. iff $f$ is surjective. For an illustrative example, consider the inclusion $f: \mathbb{N} \hookrightarrow \mathbb{Z}$, which is obviously not surjective. We find that $f^{*}: \mathbf{s H M o d}_{\mathbb{N}} \rightarrow \operatorname{sHMod}_{\mathbb{Z}}$ sends an object $X \in \operatorname{sHMod}_{\mathbb{N}}$ to $X \amalg X$, with additive inverses being provided by swapping the two terms $X$. If $g$ is a morphism, then $f^{*}(g)$ sends $\left(x, x^{\prime}\right)$ to $\left(g(x), g\left(x^{\prime}\right)\right)$. Consider $h: X \amalg X \rightarrow$ $X \amalg X$, determined by $h\left(x, x^{\prime}\right)=\left(x^{\prime}, x\right)$. If $h=f^{*}(g)$ for some $g$, then $\left(x^{\prime}, x\right)=$ $h\left(x, x^{\prime}\right)=\left(f^{*}(g)\right)\left(x, x^{\prime}\right)=\left(g(x), g\left(x^{\prime}\right)\right)$, so $g(x)=x^{\prime}$ and $g\left(x^{\prime}\right)=x$ for all $x, x^{\prime} \in X$. Hence $g$ must be constant, contradicting $h=f^{*}(g)$. Thus $f^{*}$ is not full.

Collecting previous results now establishes the following embedding theorem for pre-Hilbert categories.

3.7.8 Theorem If $\mathbf{D}$ is a pre-Hilbert category whose monoidal unit I is simple, then there is a functor $F: \mathbf{D} \rightarrow$ preHilb. It depends on the monomorphism $\mathbf{D}(I, I) \longmapsto \mathbb{C}$ of Theorem 3.6.13 by acting on objects as $F(X)=\mathbb{C} \otimes \mathbf{D}(I, X)$. The functor $F$ :

(a) is faithful if and only if I is a generator;

(b) preserves the dagger up to an isomorphism of the base field;

(c) preserves all finite limits and colimits;

(d) is monoidal. 
ProOF The only claim that does not follow directly from previous results is (c). We need to prove that extension of scalars $f^{*}$ along $f: \mathbf{D}(I, I) \longmapsto \mathbb{C}$ preserves all finite limits and colimits. This comes down to a calculation in the well-studied situation of module theory, see [9, Exercise 10.8.5].

The notion of compact object from Section 2.6 characterises when the embedding of pre-Hilbert categories takes values in finite-dimensional pre-Hilbert spaces; see also 2.6 .12 .

3.7.9 Corollary Let $\mathbf{D}$ be a compact pre-Hilbert category whose monoidal unit I is a simple generator. If $\mathbf{D}\left(I,{ }_{-}\right)$is strong monoidal, then it takes values in $\mathbf{f d H i l b . ~}$

Proof Combine Theorem 3.7.8 and Proposition 2.6.11.

3.7.10 The previous corollary opens the way to diagram chasing (see e.g. 34, Section 1.9]): to prove that a diagram commutes in a pre-Hilbert category, it suffices to prove this in pre-Hilbert spaces, where one has access to actual elements. When the embedding is strong monoidal, this partly explains the main result in [108], namely that an equation holds in all so-called traced symmetric monoidal categories if and only if it holds in finite-dimensional vector spaces; every compact closed category is traced symmetric monoidal. Moreover, in combination with the fact that the categories of finite-dimensional pre-Hilbert spaces and finite-dimensional Hilbert spaces coincide, this partly explains the main result in [198], namely that an equation holds in all dagger traced symmetric monoidal categories if and only if it holds in finite-dimensional Hilbert spaces.

Up to now we have concerned ourselves with algebraic structure only. To arrive at the category of Hilbert spaces and continuous linear maps, some analysis now comes into play. To extend the previous theorem to an embedding into the category of Hilbert spaces, we will postcompose it with the reflection preHilb ${ }^{\text {bd }} \stackrel{\perp}{\longleftrightarrow}$ Hilb induced by Cauchy completion, as discussed in 3.1 .12 We impose another axiom on the morphisms of $\mathbf{D}$ to ensure they end up in preHilb $^{\text {bd }}$ instead of preHilb. To do so we first study the positive scalars in some more detail.

3.7.11 Lemma In a pre-Hilbert category whose monoidal unit $I$ is simple, a scalar $r: I \rightarrow I$ is positive if and only if it is of the form $r=f^{\dagger} \circ f$ for some $f: I \rightarrow X$.

Proof One direction is obvious: if $r$ is positive, then by definition $r=s^{\dagger} \circ s$ for some $s: I \rightarrow I$. Conversely, suppose that $r=f^{\dagger} \circ f$ for $f: \rightarrow X$. Factorise $f$ as 
$f=i \circ e$ with $i$ dagger mono and $e$ epi.

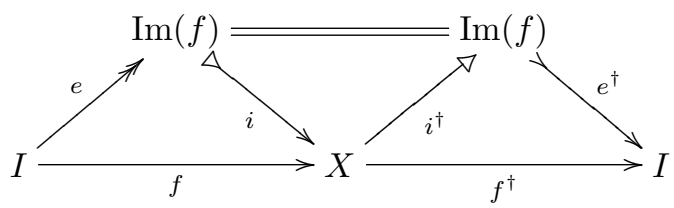

Since $I$ is simple, $e^{\dagger}$ is either zero or isomorphic. In the former case $r=f^{\dagger} f=$ $0=0^{\dagger} \circ 0$ for $0: I \rightarrow I$. In the latter case, there is an isomorphism $d: I \rightarrow I$ such that $r=f^{\dagger} \circ f=d^{\dagger} \circ d$.

3.7.12 Lemma In a pre-Hilbert category whose monoidal unit is simple, the positive scalars $R^{+}$are a sub-involutive-rig of the scalars $R$.

Proof [107, Proposition 7.5] Obviously, 0 and 1 are positive. If $r=f^{\dagger} \circ f$ and $s=g^{\dagger} \circ g$ are positive, then so are

$$
\begin{aligned}
r \bullet s & =\lambda^{-1} \circ(r \otimes s) \circ \lambda=((f \otimes g) \circ \lambda)^{\dagger} \circ((f \otimes g) \circ \lambda), \\
r+s & =\nabla \circ(r \oplus s) \circ \Delta=((f \oplus g) \circ \Delta)^{\dagger} \circ((f \oplus g) \circ \Delta),
\end{aligned}
$$

by the previous lemma. Hence $R^{+}$is a subrig of $R$. Finally, if $r=f^{\dagger} \circ f$ is positive, so is $r^{\dagger}=f^{\dagger} \circ f=r$, making $R^{+}$an involutive rig.

3.7.13 In a pre-Hilbert category whose monoidal unit is a simple generator, the relation on scalars defined by $r \leq s$ iff $s=r+p$ for some $p \in R^{+}$is a partial order: reflexivity is immediate, transitivity follows from the previous lemma, and anti-symmetry follows from the fact that $R$ is a ring.

3.7.14 Definition In a pre-Hilbert category whose monoidal unit is a simple generator, a scalar $r: I \rightarrow I$ is said to bound a morphism $g: X \rightarrow Y$ when $x^{\dagger} \circ g^{\dagger} \circ g \circ x \leq r^{\dagger} \circ x^{\dagger} \circ x \circ r$ for all $x: I \rightarrow X$. A morphism is called bounded when it has a bound.

A Hilbert category is a pre-Hilbert category whose morphisms are bounded. In the category Hilb this coincides with the usual notion of bounded map.

3.7.15 Almost by definition, the functor $\mathbf{D}\left(I,_{-}\right)$of Lemma 3.7.2 preserves boundedness of morphisms when $\mathbf{D}$ is a Hilbert category. The following lemma shows that also the extension of scalars of Theorem 3.7 .8 preserves boundedness. It is noteworthy that a combinatorial condition (boundedness) on the category $\mathbf{D}$ ensures an analytic property (continuity) of its image in preHilb, as we never even assumed a topology on the scalar field, let alone assuming completeness. 
3.7.16 Lemma Let $R$ be a commutative involutive rig, $S$ a multiplicatively cancellative commutative involutive ring, and $f: R \longmapsto S$ a monomorphism of involutive rigs. If $g: X \rightarrow Y$ is bounded in $\operatorname{sHMod}_{R}$, then $f^{*}(g)$ is bounded in $\operatorname{sHMod}_{S}$, in the notation of Lemma 3.7.6.

PROOF First, notice that $f: R \rightarrow S$ preserves the canonical order: if $r \leq r^{\prime}$, say $r+t^{\ddagger} \cdot t=r^{\prime}$ for $r, r^{\prime}, t \in R$, then $f(r)+f(t)^{\ddagger} \cdot f(t)=f\left(r+t^{\ddagger} \cdot t\right)=f\left(r^{\prime}\right)$, so $f(r) \leq f\left(r^{\prime}\right)$.

Suppose $\langle g(x) \mid g(x)\rangle_{Y} \leq r^{\ddagger} \cdot r \cdot\langle x \mid x\rangle_{X}$ for all $x \in X$ and some $r \in R$. Then $f\left(\langle g(x) \mid g(x)\rangle_{Y}\right) \leq f\left(r^{\ddagger} \cdot r \cdot\langle x \mid x\rangle_{X}\right)=f(r)^{\ddagger} f(r) f\left(\langle x \mid x\rangle_{X}\right)$ for $x \in X$. Hence for $s \in S$ :

$$
\begin{aligned}
\left\langle f^{*} g(s \otimes x) \mid f^{*} g(s \otimes x)\right\rangle_{f^{*} Y} & =\langle(\operatorname{id} \otimes g)(s \otimes x) \mid(\operatorname{id} \otimes g)(s \otimes x)\rangle_{f^{*} Y} \\
& =\langle s \otimes g(x) \mid s \otimes g(x)\rangle_{f^{*} Y} \\
& =s^{\ddagger} \cdot s \cdot f\left(\langle g(x) \mid g(x)\rangle_{Y}\right) \\
& \leq s^{\ddagger} \cdot s \cdot f(r)^{\ddagger} \cdot f(r) \cdot f\left(\langle x \mid x\rangle_{X}\right) \\
& =f(r)^{\ddagger} \cdot f(r) \cdot\langle s \otimes x \mid s \otimes x\rangle_{f^{*} X} .
\end{aligned}
$$

Because elements of the form $s \otimes x$ form a basis for $f^{*} X=S \otimes_{R} X$, we thus have

$$
\left\langle f^{*} g(z) \mid f^{*} g(z)\right\rangle_{f^{*} Y} \leq f(r)^{\ddagger} f(r)\langle z \mid z\rangle_{f^{*} X}
$$

for all $z \in f^{*} X$. In other words: $f^{*}(g)$ is bounded (namely, by $f(r)$ ).

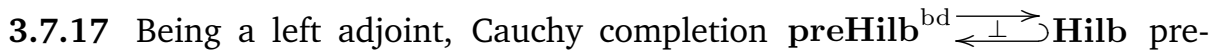
serves colimits. Because of the dagger, this means it preserves all finite limits and colimits. It is also strong monoidal, by definition of the tensor products in Hilb.

Combining the previous lemma with Theorem 3.7.8 now results in the main theorem of this section.

3.7.18 Theorem If $\mathbf{D}$ is a Hilbert category whose monoidal unit I is simple, then there is a functor $F: \mathbf{D} \rightarrow \mathbf{H i l b}$. It depends on the monomorphism $\mathbf{D}(I, I) \longmapsto \mathbb{C}$ of Theorem 3.6.13, by acting on objects as $F(X)=\mathbb{C} \otimes \mathbf{D}(I, X)$. The functor $F$ :

(a) is faithful if and only if I is a generator;

(b) preserves the dagger up to an isomorphism of the base field;

(c) preserves all finite limits and colimits;

(d) is monoidal. 
3.7.19 Notice that the embedding of the Hilbert category Hilb into itself thus constructed is (isomorphic to) the identity functor.

Theorems 3.7.8 and 3.7.18 justify taking (pre-)Hilbert categories as our categorical models for quantum theory, as indicated in Chapter 1. Except for what will be described in the next chapter, (pre-)Hilbert categories have all the structure needed to model quantum protocols. The above theorems show that they enjoy a satisfactory relation to the traditional formalism of quantum theory, namely that of Hilbert spaces.

To end this chapter, let us put (pre-)Hilbert categories and Theorem 3.7.18 into historical context by sketching related approaches.

3.7.20 Proposition 1.14 of [93] proves that any $C^{* *}$-category embeds into Hilb. Here, a C*-category is a category such that:

1. it is enriched over complex Banach spaces and linear contractions;

2. it has an antilinear dagger;

3. every $f: X \rightarrow Y$ satisfies $f^{\dagger} \circ f=0 \Rightarrow f=0$, and there is a $g: X \rightarrow X$ with $f^{\dagger} \circ f=g^{\dagger} \circ g$;

4. $\|f\|^{2}=\left\|f^{\dagger} \circ f\right\|$ for every morphism $f$.

See also Example 3.1.7. The embedding of a C*-category into Hilb uses powerful analytical methods, as it is basically an extension of the Gelfand-Naimark theorem (see Chapter (5) showing that every $\mathrm{C}^{*}$-algebra (i.e. one-object $\mathrm{C}^{*}$ category) can be realised concretely as an algebra of operators on a Hilbert space. Compare the previous definition to 3.7.1: the axioms of (pre-)Hilbert categories are much weaker. For example, nothing about the base field is built into the definition. In fact, 3.6.10 derives the fact that the base semiring is a field. For the same reason, our situation also differs from Tannakian categories [67], that are otherwise somewhat similar to our (pre-)Hilbert categories. Moreover, (pre-)Hilbert categories do not presuppose any enrichment, but derive it from prior principles.

3.7.21 A related embedding theorem is [72] (see also [105] for a categorical account). It characterises categories that are equivalent to the category of finite-dimensional unitary representations of a uniquely determined compact supergroup. Without explaining the postulates, let us mention that the categories C considered:

1. are enriched over complex vector spaces;

2. have an antilinear dagger; 
3. have finite biproducts;

4. have tensor products $(I, \otimes)$;

5. satisfy $\mathbf{C}(I, I) \cong \mathbb{C}$;

6. every projection dagger splits;

7. every object is compact.

Our Definition 3.7.1 also required 2,3, and 4 above. Furthermore, we used that $I$ is a simple generator, which is an analogue of 5 . But notice, again, that 1 above presupposes a base field $\mathbb{C}$, and enrichment over complex vector spaces, whereas (pre-)Hilbert categories do not. We will come back to point 7 in Corollary 3.7.9 below.

3.7.22 This is taken a step further by [11], which follows the "categorification" programme originating in homotopy theory [139]. A 2-Hilbert space is a category that:

1. is enriched over Hilb;

2. has an antilinear dagger;

3. is Abelian;

The category $2 \mathrm{Hilb}$ of 2-Hilbert spaces turns out to be monoidal. Hence it makes sense to define a symmetric $2-H^{*}$-algebra as a commutative monoid in $2 \mathrm{Hilb}$, in which furthermore every object is compact. Then, [11] proves that every symmetric $2-\mathrm{H}^{*}$-algebra is equivalent to a category of continuous unitary finite-dimensional representations of some compact supergroupoid. Again, the proof is basically a categorification of the Gelfand-Naimark theorem. Although the motivation for 2-Hilbert spaces is a categorification of a single Hilbert space, they resemble our (pre-)Hilbert categories, that could be seen as a characterisation of the category of all Hilbert spaces. However, there are important differences. First of all, axiom 1 above again presupposes both the complex numbers as a base field, and a nontrivial enrichment. For example, as (pre-)Hilbert categories assume no enrichment, we do not have to consider coherence with conjugation. Moreover, [11] considers only finite dimensions, whereas the category of all Hilbert spaces, regardless of dimension, is a prime example of a (pre-)Hilbert category (see also Corollary 3.7.9 below). Finally, 2-Hilbert space is an Abelian category, whereas a (pre-)Hilbert category need not be. After all, by Example 3.2.4, the Hilbert category Hilb has epic monomorphisms that are not isomorphic. 


\section{Chapter 4}

\section{Dagger kernel logic}

Whereas the previous chapters considered categorical models, which could be considered as a type theory, this chapter develops the corresponding categorical logic. By studying kernel subobjects as predicates, it turns out that there are tight connections to what is traditionally called quantum logic [30]: namely, the lattice of kernel subobjects in a dagger kernel category is always orthomodular. We establish an existential quantifier in said categories, and show that there can be no universal quantifier. This situation has hardly been considered by quantum logicians, but the categorical approach makes such structure apparent. Nonetheless, we will see that the categorical logic of dagger kernel categories is of a different nature from traditional settings studied in categorical logic: the former turns out to have a 'dynamic' character. Finally, we characterise and study the setting where the induced logic of a dagger kernel category is classical. This chapter is both a concise introduction to categorical logic and a synthesis of the results in [116] and some unpublished results obtained in cooperation with Bart Jacobs.

\subsection{Subobjects}

It could be said that categorical logic is the study of subobjects in a category. Intuitively, the latter correspond to predicates. In this section, we study socalled kernel subobjects in dagger kernel categories. These turn out to give an appropriate notion of predicate for quantum logic. For example, we will prove that such subobjects correspond to certain projections, and also to closed subobjects in a precise sence-this is reminiscent of the correspondence between projections and closed subspaces in Hilbert space theory. 
4.1.1 Recall that the collection of monomorphisms into an object $X$ in a category forms a preorder: for monos $m: M \rightarrow X$ and $n: N \rightarrow X$ we define $m \leq n$ if there is a (necessarily unique, monic) $\varphi: M \rightarrow N$ satisfying $m=n \circ \varphi$. This preorder can made into a partial order by taking equivalence classes, considering $m$ and $n$ equivalent if $m \leq n$ and $n \leq m$. Equivalently, $m$ and $n$ are equivalent if there is an isomorphism $\varphi: M \rightarrow N$ with $m=n \circ \varphi$. Such an equivalence class $[m]$ is called a subobject of $X$. The collection of subobjects of $X$ is denoted by $\operatorname{Sub}(X)$. We will often not distinguish a subobject $[m]$ from a representative $m$.

4.1.2 Example In the category Set, a subobject of an object $X$ can simply be seen as a subset of the set $X$. Hence $\operatorname{Sub}(X) \cong \mathcal{P}(X)$ in Set. In the category Mon of monoids, subobjects of $M$ correspond to submonoids of $M$. In the category Rg of rigs, subobjects of $R$ correspond to subrigs of $R$. In the category $\operatorname{Mod}_{R}$ for some rig $R$, subobjects correspond to submodules. In the category Hilb, subobjects correspond to Hilbert subspaces, i.e. closed linear subspaces.

4.1.3 Definition We say that a subobject in a dagger kernel category (cf. Definition 3.2.20 is a kernel subobject when its representatives are kernels, i.e. when every representative $m: M \longmapsto X$ of the subobject is a kernel of some morphism $f: X \rightarrow Y$. Consequently, every kernel subobject can be represented by a dagger mono (cf. [199]).

The collection $\operatorname{KSub}(X)$ of kernel subobjects of $X$ inherits the partial order of $\operatorname{Sub}(X):$ for $k: K \rightarrow X$ and $l: L \rightarrow X$ we define $k \leq l$ if there is a $\varphi: K \rightarrow L$ satisfying $k=l \circ \varphi$. By Lemma 3.4.2, $\varphi$ is also a kernel. The order on $\operatorname{KSub}(X)$ is a special case of a partial order on homsets, as in Definition 4.1.5 below.

4.1.4 Example Let us review what kernel subobjects are in the dagger kernel categories we met in Section 3.2. By Example 3.2.23, we have $\operatorname{KSub}(X) \cong \mathcal{P}(X)$ in the category Rel. By Example 3.2.24, the same holds in the subcategory PInj. Notice that in the latter category $\operatorname{KSub}(X)=\operatorname{Sub}(X)$ by Proposition 3.4.8. In the category Hilb, any subobject can be represented by isometric inclusion of a closed linear subspace and therefore is a kernel subobject by Example 3.2.25. The situation in PHilb is similar by Example 3.2.27 elements of $\operatorname{KSub}(X)$ correspond to closed subspaces of $X$ too.

4.1.5 Definition Let $f, g: X \rightrightarrows Y$ be parallel morphisms in a dagger kernel category. Factorise them as $f=i_{f} \circ m_{f} \circ\left(i_{f^{\dagger}}\right)^{\dagger}$ and $g=i_{g} \circ m_{g} \circ\left(i_{g^{\dagger}}\right)^{\dagger}$. Define $f \leq g$ if and only if there are (necessarily unique, dagger monic) $\varphi: \operatorname{Im}(f) \rightarrow$ 
$\operatorname{Im}(g)$ and $\psi: \operatorname{Im}\left(f^{\dagger}\right) \rightarrow \operatorname{Im}\left(g^{\dagger}\right)$, so that in the diagram

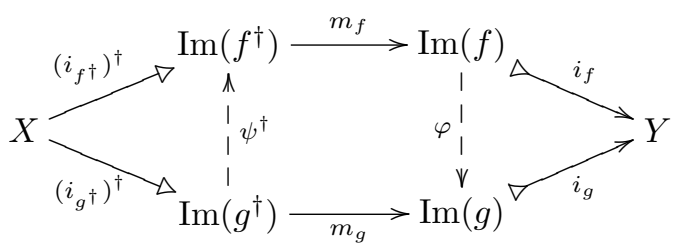

one has

$$
\psi^{\dagger} \circ\left(i_{g^{\dagger}}\right)^{\dagger}=\left(i_{f^{\dagger}}\right)^{\dagger}, \quad \varphi \circ m_{f}=m_{g} \circ \psi, \quad \varphi^{\dagger} \circ m_{g}=m_{f} \circ \psi^{\dagger}, \quad i_{g} \circ \varphi=i_{f} .
$$

4.1.6 Lemma The relation $\leq$ is a partial order on each homset of a dagger kernel category, with the zero morphism as least element.

Proof Reflexivity is easily established by taking $\varphi=$ id and $\psi=$ id. For transitivity, suppose that $f \leq g$ via $\varphi$ and $\psi$, and that $g \leq h$ via $\alpha$ and $\beta$. Then the four conditions in the previous definition are fulfilled by $\alpha \circ \varphi$ and $\psi \circ \beta$, so that $f \leq h$. Finally, for anti-symmetry, suppose that $f \leq g$ via $\varphi$ and $\psi$, and that $g \leq f$ via $\alpha$ and $\beta$.

Then $i_{f} \circ \alpha \circ \varphi=i_{g} \circ \varphi=i_{f}$, so that $\alpha \circ \varphi=$ id. Similarly, $\beta \circ \psi=$ id. By Lemma 3.4.1 $\alpha$ is a dagger mono so that $\alpha^{\dagger}=\alpha^{\dagger} \circ \alpha \circ \varphi=\varphi$. Similarly, $\beta^{\dagger}=\psi$, and therefore:

$$
\begin{aligned}
f=i_{f} \circ m_{f} \circ\left(i_{f^{\dagger}}\right)^{\dagger}=i_{f} \circ \alpha \circ \varphi \circ m_{f} \circ\left(i_{f^{\dagger}}\right)^{\dagger} & =i_{g} \circ m_{g} \circ \psi \circ\left(i_{f^{\dagger}}\right)^{\dagger} \\
& =i_{g} \circ m_{g} \circ \beta^{\dagger} \circ\left(i_{f^{\dagger}}\right)^{\dagger} \\
& =i_{g} \circ m_{g} \circ\left(i_{g^{\dagger}}\right)^{\dagger} \\
& =g .
\end{aligned}
$$

Finally, for any $f$ we have $0 \leq f$ by taking $\varphi=\psi=0$.

4.1.7 Example Let $f, g: X \rightrightarrows Y$ be morphisms in PInj. Writing out Definition 4.1.5, we see that $f \leq g$ if and only if there is a $\varphi: F \longmapsto G$ such that $f_{i}=g_{i} \circ \varphi$, in the notation of Example 3.1.5. That is, the order of Definition 4.1.5 coincides with the order on homsets of PInj induced by that of spans.

4.1.8 Example We now give explicit characterisations of the order in homsets of Rel and Hilb. An easy way to obtain these will be given in 4.2 .12 below.

In Rel we have $R \leq S$ for $R, S \subseteq X \times Y$ if and only if $S=R \cup R^{\prime}$ for some $R \subseteq X \times Y$ with $R \cap R^{\prime}=\emptyset$. That is, $R \leq S$ if and only if $R \subseteq S$ as subsets of $X \times Y$. 
In Hilb, we have $f \leq g$ for $f, g: X \rightrightarrows Y$ if and only if $g=f+f^{\prime}$ for some $f^{\prime}: X \rightarrow Y$ with $\operatorname{Im}(f)$ and $\operatorname{Im}\left(f^{\dagger}\right)$ orthogonal to $\operatorname{Im}\left(f^{\prime}\right)$ and $\operatorname{Im}\left(\left(f^{\prime}\right)^{\dagger}\right)$, respectively. (This is stronger than just $\operatorname{Im}(f) \leq \operatorname{Im}(g)$.)

4.1.9 The partial order of Definition 4.1.5 does not provide an enrichment, in general. For example, consider the morphisms $\kappa_{1}, \Delta: \mathbb{C} \rightrightarrows \mathbb{C}^{2}$ in Hilb. Then $\kappa_{1} \leq \Delta$, since $\kappa_{1}+\kappa_{2}=\Delta$ and $\operatorname{Im}\left(\kappa_{1}\right) \perp \operatorname{Im}\left(\kappa_{2}\right)$ and $\operatorname{Im}\left(\pi_{1}\right) \perp \operatorname{Im}\left(\pi_{2}\right)$. We also have $\nabla \circ \kappa_{1}=$ id and $\nabla \circ \Delta=2$. Suppose that id $\leq 2$. Then, in diagram (4.1), we must have $\varphi=\psi=$ id since $i_{\mathrm{id}}=i_{\mathrm{id}^{\dagger}}=i_{2}=i_{2^{\dagger}}=\mathrm{id}$. But then $\varphi \circ m_{\mathrm{id}}=\mathrm{id} \neq$ $2=m_{2} \circ \psi$, which contradicts Definition 4.1.5. This shows that the order is not preserved by postcomposition, in general. Nevertheless, the order is preserved under several special types of composition, as in the next lemma.

4.1.10 Lemma If parallel morphisms in a dagger kernel category satisfy $f \leq g$, then:

(a) $i \circ f \leq i \circ g$ for a dagger mono $i$;

(b) $f \circ e \leq g \circ$ e for a dagger epi $e$;

(c) $f^{\dagger} \leq g^{\dagger}$.

We omit the proof.

Before moving on to functorial properties of KSub in Section 4.4 we first study $\operatorname{KSub}(X)$ in isolation, relating it to traditional quantum logic in several ways. We start with a correspondence between kernel subobjects and certain projections.

4.1.11 Recall that a projection on an object $X$ in an arbitrary dagger kernel category is a self-adjoint idempotent morphism, i.e. a morphism $p: X \rightarrow X$ that satisfies $p^{\dagger}=p=p \circ p$. The collection $\operatorname{Proj}(X)$ of projections on $X$ carries a preorder structure in which $p \sqsubseteq q$ iff $p \circ q=p$. To see that it is in fact a partial order, suppose $p \circ q=p$ and $q \circ p=q$. Then

$$
p=p \circ q=p^{\dagger} \circ q^{\dagger}=(q \circ p)^{\dagger}=q^{\dagger}=q,
$$

so that $\sqsubseteq$ is indeed antisymmetric.

4.1.12 Proposition For any object $X$ in a dagger kernel category, there is an order isomorphism

$$
P:(\operatorname{KSub}(X), \leq) \stackrel{\cong}{\longrightarrow}(\{p \in \operatorname{Proj}(X) \mid p \leq \mathrm{id}\}, \sqsubseteq),
$$

given by $P(m)=m \circ m^{\dagger}$ and $P^{-1}(p)=\operatorname{Im}(p)$.

If zero epis are epis, then $p \leq \mathrm{id}$ holds for any $p \in \operatorname{Proj}(X)$. 
Proof Clearly, $P(m)$ is a projection satisfying $P(m) \leq$ id, by taking $\varphi=\psi=m$ in diagram (4.1). Also, the image of any morphism is by definition a kernel subobject, so that $P^{-1}$ is well-defined. Let us verify that these maps are each other's inverses. For $m \in \operatorname{KSub}(X)$, we have

$$
P^{-1} \circ P(m)=P^{-1}\left(m \circ m^{\dagger}\right)=\operatorname{Im}\left(m \circ m^{\dagger}\right)=m .
$$

Conversely, if $p \in \operatorname{Proj}(X)$, note that $p=i_{p} \circ m_{p} \circ\left(i_{p}\right)^{\dagger}$. If $p$ satisfies $p \leq$ id, then there are $\varphi, \psi: \operatorname{Im}(p) \rightrightarrows X$ with $\psi^{\dagger}=\left(i_{p}\right)^{\dagger}, \varphi \circ m_{p}=\psi, \varphi^{\dagger}=m_{p} \circ \psi^{\dagger}$ and $\varphi=i_{p}$. This yields $\psi=i_{p}$ and $m_{p}=$ id. Hence $p=i_{p} \circ\left(i_{p}\right)^{\dagger}=P(\operatorname{Im}(p))=P \circ P^{-1}(m)$.

Now let us consider the order. If $m \leq n$ as subobjects, say $m=n \circ \varphi$ for a dagger mono $\varphi$, then $m \circ m^{\dagger} \circ n \circ n^{\dagger}=n \circ \varphi \circ \varphi^{\dagger} \circ n^{\dagger} \circ n \circ n^{\dagger}=n \circ \varphi \circ \varphi^{\dagger} \circ n^{\dagger}=m \circ m^{\dagger}$, so indeed $P(m) \sqsubseteq P(n)$. Conversely, if $p \sqsubseteq q$, then $p \circ q=p$, whence $\operatorname{Im}(p \circ q)=$ $\operatorname{Im}(p)$, so that indeed $P^{-1}(p) \leq P^{-1}(q)$ by functoriality of the factorisation.

If zero epis are epis, we write for a projection $p$,

$$
i_{p} \circ e_{p}=p=p \circ p=p^{\dagger} \circ p=\left(e_{p}\right)^{\dagger} \circ\left(i_{p}\right)^{\dagger} \circ i_{p} \circ e_{p}=\left(e_{p}\right)^{\dagger} \circ e_{p} .
$$

and obtain $i_{p}=\left(e_{p}\right)^{\dagger}$. Hence $p=P\left(i_{p}\right)$ and therefore $p \leq$ id.

Compare the previous proposition to Lemma 3.4.26. We now consider the inclusion of $\mathrm{KSub}(X)$ in $\operatorname{Sub}(X)$. It will turn out that the former can be characterised as the closed subobjects of the latter, in a precise sense.

4.1.13 Let $X$ be an object in a dagger kernel category. A closure operation on $\operatorname{Sub}(X)$ [29] consists in giving an $\bar{m} \in \operatorname{Sub}(X)$ for every $m \in \operatorname{Sub}(X)$, in such a way that:

(i) $m \leq \bar{m}$;

(ii) if $m \leq n$ then $\bar{m} \leq \bar{n}$;

(iii) $\overline{\bar{m}}=\bar{m}$;

4.1.14 Lemma In a dagger kernel category, $m \mapsto \operatorname{Im}\left(\_\right)=\operatorname{ker}\left(\operatorname{coker}\left(\_\right)\right)$is a closure operation.

Proof Part (i) is easy: $\operatorname{coker}(m) \circ m=0$, so $m \leq \operatorname{ker}(\operatorname{coker}(m))$. To see (ii), suppose $m \leq n$. Then $\operatorname{coker}(n) \circ \operatorname{ker}(\operatorname{coker}(m))=0$,

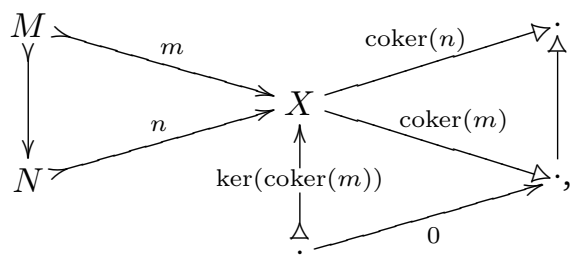


so that $\operatorname{ker}(\operatorname{coker}(m)) \leq \operatorname{ker}(\operatorname{coker}(n))$. Finally, part (iii) follows directly from Lemma 3.4.1.(c).

4.1.15 Lemma There is a reflection $\operatorname{Sub}(X) \stackrel{\operatorname{ker}\left(\operatorname{coker}\left(\_\right)\right)}{\stackrel{\perp}{\longrightarrow}} \mathrm{KSub}(X)$ for any object $X$ in a dagger kernel category where dagger monos are kernels.

ProOF We have to prove that $\operatorname{ker}(\operatorname{coker}(m)) \leq n$ iff $m \leq n$ for a mono $m$ and a dagger mono $n$. By (i) of Lemma 4.1.14 we have $m \leq \operatorname{ker}(\operatorname{coker}(m))$, proving one direction. For the converse direction, (ii) of Lemma 4.1.14 shows that $\operatorname{ker}(\operatorname{coker}(m)) \leq \operatorname{ker}(\operatorname{coker}(n))$. To finish the proof, we note that $n=$ $\operatorname{ker}(\operatorname{coker}(n))$, since dagger monos are kernels.

Finally, we relate the partial orders $\operatorname{KSub}(X)$ to the notions of simple object and generator. In doing so, we will discover that the latter notions, which might have seemed arbitrary restrictions on the category to be embedded in Chapters 2 and 3 , turn out to correspond to well-known notions in order theory.

4.1.16 Definition For elements $x, y$ of a poset, we say that $y$ covers $x$ when $x<y$ and $x \leq z<y$ implies $z=x$ (where $z<y$ if and only if $z \leq y$ and $z \neq y$ ). An element $a$ of a poset with least element 0 is called an atom when it covers 0 . Equivalently, an atom cannot be expressed as a join of strictly smaller elements. Consequently, 0 is not an atom. A poset is called atomic if for any $x \neq 0$ in it there exists an atom $a$ with $a \leq x$. Finally, a lattice is atomistic when every element is a join of atoms [64].

4.1.17 Proposition For an arbitrary object I in a dagger kernel category, the following are equivalent:

(a) $\operatorname{id}_{I}=1$ is an atom in $\operatorname{KSub}(I)$;

(b) $\operatorname{KSub}(I)=\{0,1\}$;

(c) each nonzero kernel $x: I \rightarrow X$ is an atom in $\operatorname{KSub}(X)$.

PROOF For (a) $\Rightarrow$ (b), let $m$ be a kernel into $I$. Because $m \leq \mathrm{id}_{I}$ and the latter is an atom, we have that $m=0$ or $m$ is isomorphism. $\operatorname{Thus~} \operatorname{KSub}(I)=\{0,1\}$.

To prove $(\mathrm{b}) \Rightarrow(\mathrm{c})$, suppose that $m \leq x$ for kernels $m: M \rightarrow X$ and $x: I \rightarrow$ $X$. Say $m=x \circ \varphi$ for $\varphi: M \longmapsto I$. Then $\varphi$ is a kernel by Lemma 3.4.2. Since $\operatorname{KSub}(I)=\{0,1\}$, either $\varphi$ is zero or $\varphi$ is isomorphism. Hence either $m=0$ or $m=x$ as subobjects. So $x$ is an atom. Finally, (c) $\Rightarrow$ (a) is trivial.

4.1.18 Definition If $I$ satisfies the conditions of the previous lemma, we call it a KSub-simple object. To distinguish KSub-simple objects from simple objects, 
as defined in Definition 3.6.1, we will also call the latter Sub-simple. Any Subsimple object is KSub-simple.

Similarly, let us call $I$ a KSub-generator if $f=g: X \rightrightarrows Y$ whenever $f \circ x=$ $g \circ x$ for all kernels $x: I \longmapsto X$. Any KSub-generator is a generator.

4.1.19 Lemma If a dagger kernel category $\mathrm{D}$ has a KSub-simple KSub-generator $I$, then beneath any element of $\operatorname{KSub}(X)$ lies a nonzero element of the form $x: I \rightarrow$ $X$. Hence $\operatorname{KSub}(X)$ is atomic, and its atoms are the nonzero kernels $x: I \rightarrow X$.

Proof Suppose that there is a nonzero kernel $m: M \rightarrow X$ through which no nonzero kernel $x: I \rightarrow X$ factors. If $f: I \rightarrow M$ is a kernel, then $x=m \circ f$ is a kernel that factors through $m$, so $x$ must be 0 . And since $m$ is mono, in that case also $f=0$. Hence 0 is the only kernel $I \rightarrow M$. Because $I$ is a KSub-generator, it follows that $\mathbf{D}(M, Y)=\{0\}$ for any object $Y$. But then $m=0$, which is a contradiction.

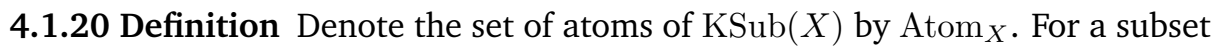
$B$ of $\operatorname{KSub}(X)$ and an element $m \in \operatorname{KSub}(X)$, write $B[m]=\{b \leq m \mid b \in B\}$.

4.1.21 Lemma If a dagger kernel category has a KSub-simple KSub-generator I, then $\operatorname{KSub}(X)$ is atomistic for any object $X$.

Proof Let $m \in \operatorname{KSub}(X)$. We show that $m$ is the least upper bound of the set $\operatorname{Atom}_{X}[m]=\{x \leq m \mid x$ atom $\}$. Obviously $m$ is an upper bound. Suppose that $x \leq n$ for all $x \in \operatorname{Atom}_{X}[m]$. We have to prove that $m \leq n$, or equivalently, $m=n \circ n^{\dagger} \circ m$. Since $I$ is a KSub-generator, it suffices to prove $m \circ y=n \circ n^{\dagger} \circ m \circ y$ for all kernels $y: I \rightarrow M$. Now, $x=m \circ y$ is an atom by Proposition 4.1.17. satisfying $x \leq m$. Hence $x \leq n$, so that $m \circ y=x=n \circ n^{\dagger} \circ x=n \circ n^{\dagger} \circ m \circ y$. $\operatorname{Thus} \operatorname{KSub}(X)$ is atomistic.

We finish this section by showing that any $\operatorname{KSub}(X)$ is a meet-semilattice. The next two sections will exhibit much richer structure.

4.1.22 Proposition For an object $X$ in a dagger kernel category, $\operatorname{KSub}(X)$ is a bounded meet-semilattice. Explicitly, its least element is $0=\operatorname{ker}\left(\operatorname{id}_{X}\right): 0 \rightarrow X$, its greatest element is $1=\mathrm{id}_{X}=\operatorname{ker}(0): X \rightarrow X$, and meets are defined by pullback.

Proof By Proposition 3.4.3, $\operatorname{KSub}(X)$ has (finite) meets-for $k, l \in \operatorname{KSub}(X)$, define $k \wedge l \in \operatorname{KSub}(X)$ as the pullback:

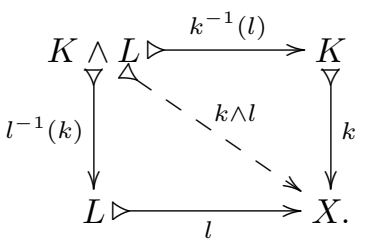




\subsection{Orthogonality}

In the category of Hilbert spaces, the existence of inner products (and hence of adjoint morphisms) induces the notion of orthogonality, with a satisfactory geometric intuition. In this section, we widen the concept of orthogonality to any dagger kernel category. The foremost consequence is that any $\operatorname{KSub}(X)$ is an orthomodular lattice, as the next section will show. This section concentrates on the fact that orthogonality makes any $\operatorname{KSub}(X)$ into a lattice.

4.2.1 Definition In a dagger category with a zero object, morphisms $f: X \rightarrow Z$ and $g: Y \rightarrow Z$ with a common codomain are called orthogonal, denoted $f \perp g$, when $g^{\dagger} \circ f=0$, or equivalently, when $f^{\dagger} \circ g=0$.

4.2.2 Example In Hilb, morphisms $f: X \rightarrow Z$ and $g: Y \rightarrow Z$ are orthogonal precisely when $\langle f(x) \mid g(y)\rangle=0$ for all $x \in X$ and $y \in Y$, i.e. when $f(x) \perp g(y)$ in the Hilbert space $Z$ for every pair of elements $x$ of $X$ and $y$ of $Y$.

4.2.3 Definition For a morphism $k: K \rightarrow X$ in a dagger kernel category, we define $k^{\perp}=\operatorname{ker}\left(k^{\dagger}\right)$. When $k$ is a kernel subobject, we call $k^{\perp}$ its orthogonal kernel subobject, and denote it by $k^{\perp}: K^{\perp} \longmapsto X$.

4.2.4 Example Let $k \in \operatorname{KSub}(X)$ be a kernel subobject in Hilb. We can represent it by (the isometric inclusion of) a closed subspace $K \subseteq X$, as in Example 3.2.4. Then $k^{\dagger}: X \rightarrow K$ is the orthogonal projection of $X$ onto $K$. Every element $x$ of the Hilbert space $X$ can be written uniquely in the form $x=y+z$, with $y \in K$ and $z \perp u$ for all $u \in K$. Then $k^{\dagger}(x)=k^{\dagger}(y+z)=y$. Hence

$$
K^{\perp}=\operatorname{ker}\left(k^{\dagger}\right)=\left\{x \in X \mid \forall_{u \in K} \cdot\langle u \mid x\rangle=0\right\} .
$$

(See [135, 2.5].) The same holds in PHilb.

Identifying a kernel subobject of $X$ in Rel with a subset $K \subseteq X$, one easily sees that $K^{\perp}=\{x \in X \mid x \notin K\}$. The same holds for PInj.

4.2.5 Lemma In a dagger kernel category one has $k^{\perp \perp}=k$ for each kernel subobject $k$. Hence every $\operatorname{KSub}(X)$ is a so-called orthoposet.

Proof Say $k=\operatorname{ker}(f)$. Then:

$$
k^{\perp \perp}=\operatorname{ker}\left(\operatorname{ker}\left(k^{\dagger}\right)^{\dagger}\right)=\operatorname{ker}(\operatorname{coker}(\operatorname{ker}(f)))=\operatorname{ker}(f)=k .
$$

4.2.6 Lemma In a dagger kernel category, the following are equivalent:

(a) f factors through $g^{\perp}$; 
(b) $f \perp g$;

(c) $g \perp f$;

(d) $g$ factors through $f^{\perp}$.

In particular, $m \leq n^{\perp}$ if and only if $n \leq m^{\perp}$. Hence there are functors

$$
\left({ }^{\perp}\right)^{\perp}: \operatorname{KSub}(X) \stackrel{\cong}{\longrightarrow} \operatorname{KSub}(X)^{\mathrm{op}} \text {. }
$$

If we interpret the elements of $\operatorname{KSub}(X)$ as predicates on $X$, then the functor $\left({ }^{\prime}\right)^{\perp}$ acts like a negation on predicates. In some ways it indeed behaves as expected of a negation, as in the following lemma.

4.2.7 Lemma In a dagger kernel category, the functor $\perp: \operatorname{KSub}(X)^{\mathrm{op}} \rightarrow \operatorname{KSub}(X)$ is an equivalence of categories. In particular, it is both left and right adjoint to its opposite $\perp^{\mathrm{op}}: \operatorname{KSub}(X) \rightarrow \operatorname{KSub}(X)^{\mathrm{op}}$.

Proof This means that $m^{\perp} \leq n$ iff $n^{\perp} \leq m$, which holds as $\perp$ is involutive.

But the properties of $\left(\_\right)^{\perp}$ resemble those of a negation only so far, as the following corollary to Proposition 3.4.3 exhibits.

4.2.8 Corollary The following diagram is a pullback:

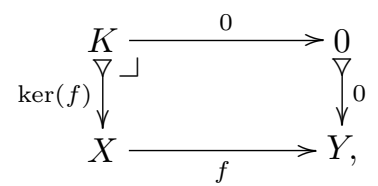

showing that, logically speaking, falsum-i.e. the bottom element $0 \in \mathrm{KSub}(Y)$-is in general not preserved under substitution. Also, negation $\left({ }_{-}\right)^{\perp}$ does not commute with substitution, because $1=0^{\perp}$ and $f^{-1}(1)=1$.

4.2.9 In Proposition 4.1.22 we saw that every $\operatorname{KSub}(X)$ is a meet-semilattice in a dagger kernel category. Combining this with the functor $\left({ }_{-}\right)^{\perp}: \operatorname{KSub}(X)^{\mathrm{op}} \rightarrow$ $\operatorname{KSub}(X)$, we find that $\operatorname{KSub}(X)$ is a lattice, by defining $k \vee l=\left(k^{\perp} \wedge l^{\perp}\right)^{\perp}$. Joins are not necessarily preserved by pullback along a morphism $f$, but we do have:

$$
k \vee k^{\perp}=\left(k^{\perp} \wedge k^{\perp \perp}\right)^{\perp}=\left(k^{\perp} \wedge k\right)^{\perp}=0^{\perp}=1 .
$$

In other words, $\operatorname{KSub}(X)$ is an orthocomplemented lattice.

As an intermezzo, we can now characterise the order of Definition 4.1.5 in terms of biproducts, if these are available. The following two results also apply to PInj, with its coaffine structure of disjoint union instead of $\oplus$. For simplicity, we only state them using biproducts. 
4.2.10 Lemma For parallel morphisms $f, g, h$ in a dagger kernel category with dagger biproducts:

(a) $h \perp(f+g)$ if and only if $h \perp f$ and $h \perp g$;

(b) If $f+g=0$ then $f=g=0$;

(c) If $f+g=f$ then $g=0$.

Proof For (a), observe that $h \perp f$ if and only if $i_{h} \perp i_{f}$. Hence $h \perp f$ and $h \perp g$ if and only if $i_{f}^{\dagger} \circ i_{h}=0$ and $i_{g}^{\dagger} \circ i_{h}=0$. This is equivalent to

$$
i_{f+g}^{\dagger} \circ i_{h}=\left[i_{f}, i_{g}\right]^{\dagger} \circ i_{h}=\left\langle i_{f}^{\dagger}, i_{g}^{\dagger}\right\rangle \circ i_{h}=0,
$$

and hence to $h \perp(f+g)$.

For (b), assume that $f+g=0$. Then $\left[i_{f}, i_{g}\right] \circ\left\langle e_{f}, e_{g}\right\rangle=f+g=0$. Since $\left[i_{f}, i_{g}\right]$ is monic, this entails $\left\langle e_{f}, e_{g}\right\rangle=0$. Hence $e_{f}=e_{g}=0$ and $f=g=0$.

For (c), assume that $f+g=f$. Then

$$
\left[i_{f}, i_{g}\right] \circ\left\langle e_{f}, e_{g}\right\rangle=f+g=f=i_{f} \circ e_{f}=\left[i_{f}, i_{g}\right] \circ \kappa_{1} \circ e_{f},
$$

so that $\left\langle e_{f}, e_{g}\right\rangle=\kappa_{1} \circ e_{f}$. But then $e_{g}=\pi_{2} \circ\left\langle e_{f}, e_{g}\right\rangle=\pi_{2} \circ \kappa_{1} \circ e_{f}=0$, whence $g=0$.

4.2.11 Proposition If a dagger category $\mathbf{D}$ has dagger biproducts, its homsets carry a partial order, in which $f \leq g$ if and only if there is an $f^{\prime}$ such that $f \perp f^{\prime}$ and $f^{\dagger} \perp\left(f^{\prime}\right)^{\dagger}$ and $f+f^{\prime}=g$. This order coincides with that of Definition 4.1 .5 when there is a (necessarily unique dagger) isomorphism $\varphi: K \oplus K^{\perp} \rightarrow X$ with $\varphi \circ \kappa_{1}=k$ and $\varphi \circ \kappa_{2}=k^{\perp}$ for every $k \in \operatorname{KSub}(X)$.

PROOF Reflexivity is easy: $f \leq f$ by picking $f^{\prime}=0$. For transitivity, assume $f \leq g$ and $g \leq h$, say by $f+f^{\prime}=g$ and $g+g^{\prime}=h$ with $f \perp f^{\prime}, f^{\dagger} \perp\left(f^{\prime}\right)^{\dagger}, g \perp g^{\prime}$ and $g^{\dagger} \perp\left(g^{\prime}\right)^{\dagger}$. Picking $f^{\prime \prime}=f^{\prime}+g^{\prime}$ yields $f+f^{\prime \prime}=h$. By Lemma 4.2.10(a), we obtain $f \perp f^{\prime \prime}$ and $f^{\dagger} \perp\left(f^{\prime \prime}\right)^{\dagger}$, whence $f \leq h$. For anti-symmetry, assume that $f \leq g$ and $g \leq f$, say by $f+f^{\prime}=g$ and $g+g^{\prime}=f$ with $f \perp f^{\prime}, f^{\dagger} \perp\left(f^{\prime}\right)^{\dagger}, g \perp g^{\prime}$ and $g^{\dagger} \perp\left(g^{\prime}\right)^{\dagger}$. Then $f=f+\left(f^{\prime}+g^{\prime}\right)$, so that $f^{\prime}+g^{\prime}=0$ by Lemma 4.2.10(c), and so $f^{\prime}=g^{\prime}=0$ by Lemma 4.2.10(b). But then $f=g$. Thus $\leq$ is a partial order.

To show that this partial coincides with the one of Definition 4.1.5, first assume $f+f^{\prime}=g$, where $f \perp f^{\prime}$ and $f^{\dagger} \perp\left(f^{\prime}\right)^{\dagger}$. The factorisation of $f+f^{\prime}$ yields 
a diagram of the form (4.1), namely:

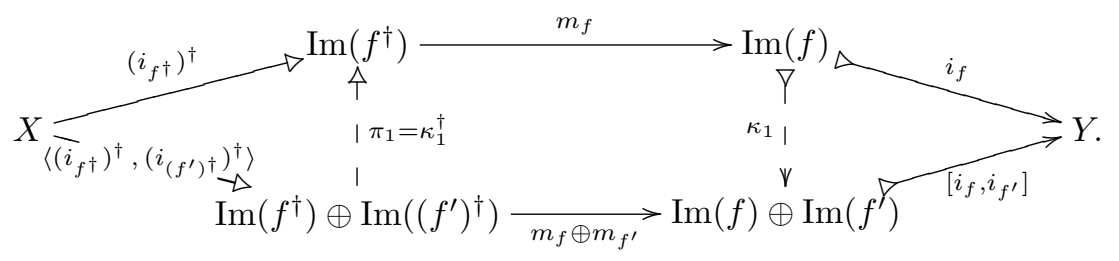

Conversely, assume $\varphi, \psi$ as in diagram (4.1), so that there are isomorphisms $\left[\varphi, \varphi^{\perp}\right]: \operatorname{Im}(f) \oplus \operatorname{Im}(f)^{\perp} \rightarrow \operatorname{Im}(g)$, and $\left[\psi, \psi^{\perp}\right]: \operatorname{Im}\left(f^{\dagger}\right) \oplus \operatorname{Im}\left(f^{\dagger}\right)^{\perp} \rightarrow \operatorname{Im}\left(g^{\dagger}\right)$. Since $\varphi^{\dagger} \circ m_{g}=m_{f} \circ \psi^{\dagger}$, there is an $n$ making the following diagram commute:

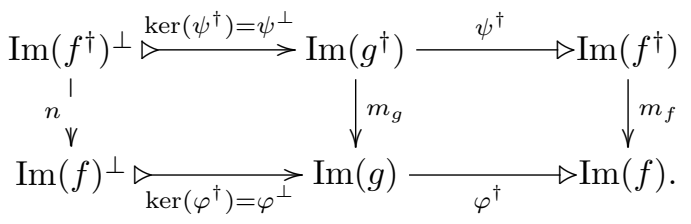

Hence

$$
\begin{aligned}
{\left[\varphi, \varphi^{\perp}\right] \circ\left(m_{f} \oplus n\right) \circ\left[\psi, \psi^{\perp}\right]^{\dagger} } & =\left[\varphi \circ m_{f}, \varphi^{\perp} \circ n\right] \circ\left[\psi, \psi^{\perp}\right]^{\dagger} \\
& =m_{g} \circ\left[\psi, \psi^{\perp}\right] \circ\left[\psi, \psi^{\perp}\right]^{\dagger}=m_{g} .
\end{aligned}
$$

Now put

$$
f^{\prime}=\left(X \stackrel{\left(i_{g^{\dagger}}\right)^{\dagger}}{\longrightarrow} \operatorname{Im}\left(g^{\dagger}\right) \stackrel{\left(\psi^{\perp}\right)^{\dagger}}{\longrightarrow} \operatorname{Im}\left(f^{\dagger}\right)^{\perp} \stackrel{n}{\longrightarrow} \operatorname{Im}(f)^{\perp} \stackrel{\varphi^{\perp}}{\longrightarrow} \operatorname{Im}(g) \stackrel{i_{g}}{\longrightarrow} Y\right) .
$$

Then

$$
\begin{aligned}
f^{\dagger} \circ f^{\prime} & =f^{\dagger} \circ i_{g} \circ \varphi^{\perp} \circ n \circ\left(\psi^{\perp}\right)^{\dagger} \circ\left(i_{g^{\dagger}}\right)^{\dagger} \\
& =\left(e_{f}\right)^{\dagger} \circ\left(i_{f}\right)^{\dagger} \circ i_{g} \circ \varphi^{\perp} \circ n \circ\left(\psi^{\perp}\right)^{\dagger} \circ\left(i_{g^{\dagger}}\right)^{\dagger} \\
& =\left(e_{f}\right)^{\dagger} \circ\left(i_{f}\right)^{\dagger} \circ i_{g} \circ \operatorname{ker}\left(\left(i_{f}\right)^{\dagger} \circ i_{g}\right) \circ n \circ\left(\psi^{\perp}\right)^{\dagger} \circ\left(i_{g^{\dagger}}\right)^{\dagger} \\
& =\left(e_{f}\right)^{\dagger} \circ 0 \circ n \circ\left(\psi^{\perp}\right)^{\dagger} \circ\left(i_{g^{\dagger}}\right)^{\dagger}=0,
\end{aligned}
$$

whence $f \perp f^{\prime}$. Analogously $f^{\dagger} \perp\left(f^{\prime}\right)^{\dagger}$. Finally, we verify that $f+f^{\prime}=g$ :

$$
\begin{aligned}
g & =i_{g} \circ m_{g} \circ\left(i_{g^{\dagger}}\right)^{\dagger} \\
& =i_{g} \circ\left[\varphi, \varphi^{\perp}\right] \circ\left(m_{f} \oplus n\right) \circ\left[\psi, \psi^{\perp}\right]^{\dagger} \circ\left(i_{g^{\dagger}}\right)^{\dagger} \\
& =\left[i_{g} \circ \varphi, i_{g} \circ \varphi^{\perp}\right] \circ\left(m_{f} \oplus n\right) \circ\left\langle\psi^{\dagger} \circ\left(i_{g^{\dagger}}\right)^{\dagger},\left(\psi^{\perp}\right)^{\dagger} \circ\left(i_{g^{\dagger}}\right)^{\dagger}\right\rangle \\
& =\left(i_{f} \circ m_{f} \circ\left(i_{f^{\dagger}}\right)^{\dagger}\right)+\left(i_{g} \circ \varphi^{\perp} \circ n \circ\left(\psi^{\perp}\right)^{\dagger} \circ\left(i_{g^{\dagger}}\right)^{\dagger}\right)=f+f^{\prime}
\end{aligned}
$$


4.2.12 Since Rel and Hilb both have dagger biproducts satisfying the requirement of the previous proposition, we have an explicit characterisation of the order on their homsets, justifying Example 4.1.8.

We finish this section by showing that the existence of directed colimits suffices for the kernel subobject lattice to be complete.

4.2.13 Recall that a preorder is directed when every two elements have a common upper bound; a directed colimit is a colimit of a directed preorder considered as a diagram.

The following lemma is a special case of [33, Proposition 2.16.3].

4.2.14 Lemma If a category $\mathbf{C}$ has directed colimits and $X$ is an object in it, then the slice category $\mathbf{C} / X$ has directed colimits as well.

Proof Let $\left(k_{i}\right)_{i \in I}$ be a directed diagram in $\mathbf{C} / X$. Then $\left(K_{i}\right)_{i \in I}$ is a directed diagram in $\mathbf{C}$; let $c_{i}: K_{i} \rightarrow K$ be a colimit.

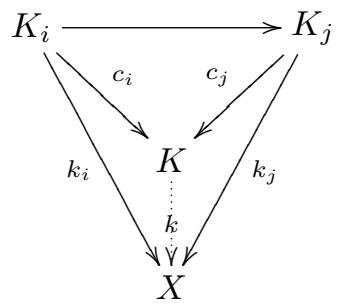

Then there is a morphism $k: K \rightarrow X$ such that $k_{i}=k \circ c_{i}$, i.e. there is a cone $c_{i}: k_{i} \rightarrow k$ in $\mathbf{C} / X$.

Suppose that $d_{i}: k_{i} \rightarrow l$ is a cone in $\mathbf{C} / X$. That is, suppose that morphisms $d_{i}: K_{i} \rightarrow L$ satisfy $k_{i}=l \circ d_{i}$. Then $\left(d_{i}\right)_{i \in I}$ forms a cone in $\mathbf{C}$, so there is a $\varphi: K \rightarrow L$ with $d_{i}=\varphi \circ c_{i}$. Hence $l \circ \varphi \circ c_{i}=k \circ c_{i}$ for all $i \in I$. Since the $c_{i}$ are jointly epimorphic, we have $l \circ \varphi=k$. Hence $l \circ \varphi=k$ in $\mathbf{C} / X$, and the diagram $\left(k_{i}\right)_{i \in I}$ indeed has a colimit in $\mathbf{C} / X$, namely $c_{i}: k_{i} \rightarrow k$.

The previous lemma specialises to kernel subobjects, as follows.

4.2.15 Lemma If a dagger kernel category $\mathrm{D}$ has directed colimits, then $\operatorname{KSub}(X)$ has directed colimits for every $X \in \mathbf{D}$.

Proof Lemma 4.1 .15 extends to a reflection $\mathbf{D} / X_{\longleftrightarrow}^{\stackrel{\operatorname{Im}\left(\_\right)}{\perp}} \operatorname{LSub}(X)$. Indeed, for $f: Y \rightarrow X$ in $\mathbf{D} / X$ and $k: K \longmapsto X$ in $\operatorname{KSub}(X)$, we find that morphisms $d: i_{f} \rightarrow k$ in $\operatorname{KSub}(X)$ correspond bijectively to morphisms $\varphi: f \rightarrow k$ in 
$\mathbf{D} / X$ by using diagonal fill-in on the following diagram:

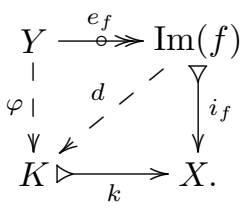

Let a directed diagram in $\operatorname{KSub}(X)$ be given. Regarded as a diagram in $\mathbf{D} / X$, it has a colimit $c$. Because $\operatorname{Im}\left(\_\right)$is a left adjoint, $\operatorname{Im}(c)$ is a colimit for the diagram in $\operatorname{KSub}(X)$. Hence $\operatorname{KSub}(X)$ has directed colimits if $\mathbf{D} / X$ does, which is the case if $\mathbf{D}$ has directed colimits by the previous lemma.

4.2.16 Proposition If a dagger kernel category $\mathrm{D}$ has directed colimits, $\operatorname{KSub}(X)$ is a complete lattice for every $X \in \mathbf{D}$.

PROOF Combine the previous lemma with the fact that a lattice is complete if it has directed joins ([130, Lemma I.4.1], or [137, Lemma 2.12]).

\subsection{Orthomodularity}

This section shows that any $\operatorname{KSub}(X)$ is an orthomodular lattice, thereby justifying the study of dagger kernel categories in the context of quantum logic. We then study the totality of orthomodular lattices as the objects of a particular dagger kernel category.

4.3.1 Definition An orthocomplemented lattice is called an orthomodular lattice when it satisfies $k \vee\left(k^{\perp} \wedge l\right)=l$, (or, equivalently, $k=l \wedge\left(l^{\perp} \vee k\right)$ ) if $k \leq$ $l$ [136]. This is the notion that has come to be known as "the logic of quantum mechanics" after the work of Garett Birkhoff and John von Neumann [30].

4.3.2 Lemma If $k: K \longmapsto X$ is a kernel subobject in a dagger kernel category, then $k \circ\left(_{-}\right): \operatorname{KSub}(K) \rightarrow \mathrm{KSub}(X)$ is left adjoint to $k^{-1}\left({ }_{-}\right)$, and therefore preserves joins.

PROoF For kernel subobjects $l, m$ one has $l \leq \operatorname{ker}(\operatorname{coker}(m) \circ k)=k^{-1}(m)$ if and only if $\operatorname{coker}(m) \circ k \circ l=0$. But the latter holds if and only if $k \circ l \leq$ $\operatorname{ker}(\operatorname{coker}(m))=m$ by Lemma 3.4.1(c).

4.3.3 Theorem For any object $X$ in a dagger kernel category, $\operatorname{KSub}(X)$ is an orthomodular lattice. 
ProOF We first prove that if kernel subobjects $k, l$ of $X$ in a dagger kernel category satisfy $k \leq l$, say via $\varphi$ with $l \circ \varphi=k$, one has pullbacks

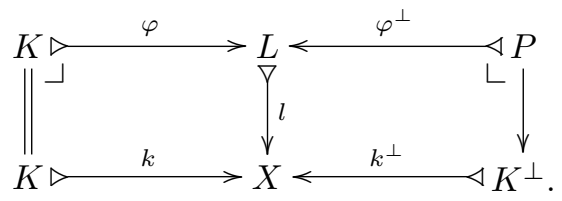

The square on the left is obviously a pullback. For the one on the right we use a simple calculation, following Proposition 3.4 .3 .

$$
\begin{aligned}
l^{-1}\left(k^{\perp}\right) & =\operatorname{ker}\left(\operatorname{coker}\left(k^{\perp}\right) \circ l\right) \\
& =\operatorname{ker}\left(\operatorname{coker}\left(\operatorname{ker}\left(k^{\dagger}\right)\right) \circ l\right) \\
& =\operatorname{ker}\left(k^{\dagger} \circ l\right) \\
& \stackrel{*}{=} \operatorname{ker}\left(\varphi^{\dagger}\right) \\
& =\varphi^{\perp},
\end{aligned}
$$

where the marked equation holds because $l \circ \varphi=k$, so that $\varphi=l^{\dagger} \circ l \circ \varphi=l^{\dagger} \circ k$, and therefore $\varphi^{\dagger}=k^{\dagger} \circ l$. Then:

$$
k \vee\left(k^{\perp} \wedge l\right)=(l \circ \varphi) \vee\left(l \circ \varphi^{\perp}\right) \stackrel{*}{=} l \circ\left(\varphi \vee \varphi^{\perp}\right)=l \circ \mathrm{id}=l,
$$

where the marked equation holds because by Lemma 4.3.2. Hence $\operatorname{KSub}(X)$ is an orthomodular lattice.

4.3.4 In connection to 3.7.15, Lemma 4.1.14 and Lemma 4.1.15, a relevant fact is that a pre-Hilbert space is complete if and only if its lattice of closed subspaces is orthomodular [7]. However, preHilb is not a dagger kernel category, for if $f: X \rightarrow Y$, then $\{x \in X \mid f(x)=0\}$ is again an object of preHilb, but the its inclusion into $X$ is not necessarily adjointable. Hence the previous theorem fortunately does not imply that every pre-Hilbert space is a Hilbert space.

The following definition collects all orthomodular lattices into a category, choosing the morphisms such that it becomes a dagger kernel category. The rest of this section studies that category.

4.3.5 Definition We denote by OMLatGal the full subcategory of InvGal of Example 3.1 .9 consisting of orthomodular lattices. Let us write out the morphisms, recalling Example 3.1.9. A morphism $f: X \rightarrow Y$ in OMLatGal is an antitone Galois connection, i.e. a pair of functions $f_{*}: X^{\mathrm{op}} \rightarrow Y$ and $f^{*}: Y \rightarrow$ $X^{\mathrm{op}}$ such that: 
- $x \leq f^{*}(y)$ if and only if $y \leq f_{*}(x)$;

- $f_{*}(x) \geq f_{*}\left(x^{\prime}\right)$ when $x \leq x^{\prime}$;

- $f^{*}(y) \geq f^{*}\left(y^{\prime}\right)$ when $y \leq y^{\prime}$.

The identity morphism on $X$ is given by the pair $(\perp, \perp)$, and composition is determined by

$$
\begin{aligned}
& (g \circ f)_{*}=g_{*} \circ \perp \circ f_{*}, \\
& (g \circ f)^{*}=f^{*} \circ \perp \circ g^{*} .
\end{aligned}
$$

The components $f_{*}: X^{\mathrm{op}} \rightarrow Y$ and $f^{*}: Y \rightarrow X^{\text {op }}$ of a morphism $f: X \rightarrow$ $Y$ in OMLatGal are not required to preserve any structure, but the Galois connection yields that $f_{*}$ preserves meets, as a right adjoint, and therefore sends joins in $X$ (meets in $X^{\mathrm{op}}$ ) to meets in $Y$. Dually, $f^{*}$ sends joins in $Y$ to meets in $X$.

4.3.6 The category OMLatGal inherits the dagger from InvGal:

$$
\left(f_{*}, f^{*}\right)^{\dagger}=\left(f^{*}, f_{*}\right)
$$

A morphism $f: X \rightarrow Y$ is a dagger mono in OMLatGal precisely when it safisfies $f^{*}\left(f_{*}(x)^{\perp}\right)=x^{\perp}$ for all $x \in X$, because $\operatorname{id}^{*}(x)=x^{\perp}=\operatorname{id}_{*}(x)$ and

$$
\left(f^{\dagger} \circ f\right)^{*}(x)=f^{*}\left(f_{*}(x)^{\perp}\right)=\left(f^{\dagger} \circ f\right)_{*}(x) .
$$

4.3.7 Lemma Let $f, g: X \rightrightarrows Y$ be morphisms of OMLatGal. One has $f=g$ if either $f_{*}=g_{*}$ or $f^{*}=g^{*}$.

Proof We prove that $f^{*}=g^{*}$ if $f_{*}=g_{*}$. For all $x \in X$ and $y \in Y$,

$$
x \leq f^{*}(y) \Longleftrightarrow y \leq f_{*}(x)=g_{*}(x) \Longleftrightarrow x \leq g^{*}(y) .
$$

Given $y$, this holds for all $x$, and so in particular for $x=f^{*}(y)$ and $x=g^{*}(y)$, which yields $f^{*}(y)=g^{*}(y)$.

4.3.8 Lemma Let $X$ be an orthomodular lattice, and $a \in X$.

(a) The (principal) downset $\downarrow a=\{u \in X \mid u \leq a\}$ is again an orthomodular lattice, with order and meets as in $X$, and with orthocomplement $\perp_{a}$ given by $u^{\perp_{a}}=a \wedge u^{\perp}$, where $\perp$ is the orthocomplement of $X$.

(b) There is a dagger mono $\downarrow a \triangleright \longrightarrow X$ in OMLatGal, for which we also write $a$, determined by $a_{*}(u)=u^{\perp}$ and $a^{*}(x)=a \wedge x^{\perp}$. 
Proof For (a), let $u \in \downarrow a$. Then

$$
u^{\perp_{a} \perp_{a}}=a \wedge\left(a \wedge u^{\perp}\right)^{\perp}=a \wedge\left(a^{\perp} \vee u\right)=u,
$$

by orthomodularity, since $u \leq a$. We thus obtain a morphism in OMLatGal, because for arbitrary $u \in \downarrow a$ and $x \in X$ :

$$
x \leq a_{*}(u)=u^{\perp} \Longleftrightarrow u \leq x^{\perp} \Longleftrightarrow u \leq a \wedge x^{\perp}=a^{*}(x) .
$$

This map $a: \downarrow a \rightarrow X$ is a dagger mono since

$$
a^{*}\left(a_{*}(u)^{\perp}\right)=a^{*}\left(u^{\perp \perp}\right)=a^{*}(u)=a \wedge u^{\perp}=u^{\perp_{a}} .
$$

Later, in Proposition 4.6.5, we shall see that the maps $\downarrow a \triangleright \longrightarrow X$ of the previous lemma are precisely the kernels in the category OMLatGal. But we first show that this category has kernels in the first place.

4.3.9 The category OMLatGal has a zero object $\underline{0}$, namely the one-element orthomodular lattice $\{*\}$. We can write its unique element as $*=0=1$. Let us show that the lattice $\underline{0}$ is indeed a final object in OMLatGal. Let $X$ be an arbitrary orthomodular lattice. The only function $f_{*}: X \rightarrow \underline{0}$ is $f_{*}(x)=1$. We will show that it has a left adjoint $f^{*}: \underline{0} \rightarrow X$ defined by $f^{*}(1)=1$ :

$$
\begin{array}{ll}
\frac{f^{*}(1)=1 \leq x}{\frac{1 \geq x}{1 \geq x}} & \text { in } X^{\text {op }} \\
\overline{\overline{1 \leq 1=f_{*}(x)}} & \text { in } \underline{0} .
\end{array}
$$

Likewise, the unique morphism $g: \underline{0} \rightarrow Y$ is given by $g_{*}(1)=1$ and $g^{*}(y)=1$. Hence the zero morphism $z: X \rightarrow Y$ is determined by $z_{*}(x)=1$ and $z^{*}(y)=1$.

4.3.10 Proposition The category OMLatGal is a dagger kernel category. Explicitly, the kernel of $f: X \rightarrow Y$ is $k: \downarrow k \rightarrow X$, where $k=f^{*}(1)$, as in Lemma 4.3.8

PROOF The composition $f \circ k$ is the zero morphism $\downarrow k \rightarrow Y$. First, for $u \in$ $\downarrow f^{*}(1)$ :

$$
(f \circ k)_{*}(u)=f_{*}\left(k_{*}(u)^{\perp}\right)=f_{*}(u)=1,
$$

because $u \leq f^{*}(1)$ (in $X$ ) and so $f_{*}(u) \leq 1$ (in $Y$ ). And for $y \in Y$ :

$$
(f \circ k)^{*}(y)=k^{*}\left(f^{*}(y)^{\perp}\right)=f^{*}(y) \wedge f^{*}(1)=f^{*}(y \vee 1)=f^{*}(1)=k=1_{\downarrow k} .
$$

Now suppose that $g \circ k$ equals the zero morphism for $g: Z \rightarrow X$. Then $f_{*} \circ \perp \circ g_{*}=1$ and $g^{*} \circ \perp \circ f^{*}=1$. Hence for $z \in Z$ we have $1 \leq f_{*}\left(g_{*}(z)^{\perp}\right)$, 
so $g_{*}(z)^{\perp} \leq f^{*}(1)=k$. Define $h_{*}: Z^{\mathrm{op}} \rightarrow \downarrow k$ by $h_{*}(z)=g_{*}(z) \wedge k$, and define $h^{*}: \downarrow k \rightarrow Z^{\mathrm{op}}$ by $h^{*}(u)=g^{*}(u)$. Then $h^{*} \dashv h_{*}$ since for $u \leq k$ and $z \in Z$ :

$$
\begin{array}{cl}
\frac{h^{*}(u)=g^{*}(u) \leq z}{u \leq g_{*}(z)} & \text { in } Z^{\text {op }} \\
\overline{\overline{u \leq g_{*}(z) \wedge k=h_{*}(z)}} & \text { in } K \\
\text { in } K,
\end{array}
$$

whence $h$ is a well-defined morphism of OMLatGal. It satisfies

$$
\begin{aligned}
(k \circ h)_{*}(z) & =k_{*}\left(h_{*}(z)^{\perp \downarrow k}\right) \\
& =k_{*}\left(\left(g_{*}(z) \wedge k\right)^{\perp \downarrow k}\right) \\
& =\left(\left(g_{*}(z) \wedge k\right)^{\perp \downarrow k} \wedge k\right)^{\perp} \\
& =\left(\left(g_{*}(z) \wedge k\right)^{\perp} \wedge k \wedge k\right)^{\perp} \\
& =\left(g_{*}(z) \wedge k\right) \vee k^{\perp} \\
& =g_{*}(z)
\end{aligned}
$$

by orthomodularity, since $k=f^{*}(1)^{\perp} \leq g_{*}(z)$ because $g_{*}(z)^{\perp} \leq f^{*}(1)=k$, which follows from $1 \leq f_{*}\left(g_{*}(z)^{\perp}\right)$. Hence $h$ is a mediating morphism satisfying $k \circ h=g$. It is the unique such morphism, since $k$ is a dagger mono and hence a mono, as we will now show to finish the proof: for $u \in K$ we have $k^{*}\left(k_{*}(u)^{\perp}\right)=$ $k^{*}(u)=u^{\perp_{K}}$.

4.3.11 Proposition The category OMLatGal has (finite) dagger biproducts. Explicitly, $X_{1} \oplus X_{2}$ is the Cartesian product of orthomodular lattices, and the coprojection $\kappa_{1}: X_{1} \rightarrow X_{1} \oplus X_{2}$ is defined by $\left(\kappa_{1}\right)_{*}(x) \mapsto\left(x^{\perp}, 1\right)$ and $\left(\kappa_{1}\right)^{*}(x, y)=x^{\perp}$. The projections are given by $\pi_{i}=\left(\kappa_{i}\right)^{\dagger}$.

Proof Let us first verify that $\kappa_{1}$ is a well-defined morphism of OMLatGal, i.e. that $\left(\kappa_{1}\right)^{*} \dashv\left(\kappa_{1}\right)_{*}$ :

$$
\begin{array}{cl}
\frac{\kappa_{1}^{*}(x, y)=x^{\perp} \leq z}{(x, y) \leq\left(z^{\perp}, 1\right)=\left(\kappa_{1}\right)_{*}(z)} & \text { in } X \oplus Y .
\end{array}
$$

Since $\left(\kappa_{1}\right)^{*}\left(\left(\kappa_{1}\right)_{*}(x)^{\perp}\right)=\left(\kappa_{1}\right)^{*}\left(\left(x^{\perp}, 1\right)^{\perp}\right)=\left(\kappa_{1}\right)^{*}(x, 0)=x^{\perp}$, one finds that $\kappa_{1}$ is a dagger mono. Likewise, there is a dagger mono $\kappa_{2}: X_{2} \rightarrow X_{1} \oplus X_{2}$. For $i \neq j$, one finds that $\left(\kappa_{j}\right)^{\dagger} \circ \kappa_{i}$ is the zero morphism.

To show that $X_{1} \oplus X_{2}$ is indeed a coproduct, let morphisms $f_{i}: X_{i} \rightarrow Y$ be 
given. We then define the cotuple $\left[f_{1}, f_{2}\right]: X_{1} \oplus X_{2} \rightarrow Y$ by

$$
\begin{aligned}
{\left[f_{1}, f_{2}\right]_{*}\left(x_{1}, x_{2}\right) } & =\left(f_{1}\right)_{*}\left(x_{1}\right) \wedge\left(f_{2}\right)_{*}\left(x_{2}\right), \\
{\left[f_{1}, f_{2}\right]^{*}(y) } & =\left(f_{1}^{*}(y), f_{2}^{*}(y)\right) .
\end{aligned}
$$

Indeed $\left[f_{1}, f_{2}\right]^{*} \dashv\left[f_{1}, f_{2}\right]_{*}$, making it a well-defined morphism:

$$
\begin{array}{cl}
\frac{\left[f_{1}, f_{2}\right]^{*}(y)=\left(f_{1}^{*}(y), f_{2}^{*}(y)\right) \leq\left(x_{1}, x_{2}\right)}{\underline{f_{i}^{*}(y) \leq x_{i}}} & \text { in }\left(X_{1} \oplus X_{2}\right)^{\mathrm{op}} \\
\overline{\overline{y \leq\left(f_{i}\right)_{*}\left(x_{i}\right)}} & \text { in } X_{i}^{\mathrm{op}} \\
\overline{y \leq\left(f_{1}\right)_{*}\left(x_{1}\right) \wedge\left(f_{2}\right)_{*}\left(x_{2}\right)=\left[f_{1}, f_{2}\right]_{*}\left(x_{1}, x_{2}\right)} & \text { in } Y \\
\text { in } Y .
\end{array}
$$

Then

$$
\begin{aligned}
\left(\left[f_{1}, f_{2}\right] \circ \kappa_{1}\right)_{*}(x) & =\left[f_{1}, f_{2}\right]_{*}\left(\left(\kappa_{1}\right)_{*}(x)^{\perp}\right) \\
& =\left[f_{1}, f_{2}\right]_{*}\left(\left(x^{\perp}, 1\right)^{\perp}\right) \\
& =\left(f_{1}\right)_{*}(x) \wedge\left(f_{2}\right)_{*}(0) \\
& =\left(f_{1}\right)_{*}(x) \wedge 1=\left(f_{1}\right)_{*}(x),
\end{aligned}
$$

so that $\left[f_{1}, f_{2}\right] \circ \kappa_{1}=f_{1}$. Likewise, $\left[f_{1}, f_{2}\right] \circ \kappa_{2}=f_{2}$. Moreover, if $g: X_{1} \oplus X_{2} \rightarrow$ $Y$ also satisfies $g \circ \kappa_{i}=f_{i}$, then:

$$
\begin{aligned}
{\left[f_{1}, f_{2}\right]_{*}\left(x_{1}, x_{2}\right) } & =\left(f_{1}\right)_{*}\left(x_{1}\right) \wedge\left(f_{2}\right)_{*}\left(x_{2}\right) \\
& =g_{*}\left(\left(\kappa_{1}\right)_{*}\left(x_{1}\right)^{\perp}\right) \wedge g_{*}\left(\left(\kappa_{2}\right)_{*}\left(x_{2}\right)^{\perp}\right) \\
& =g_{*}\left(\left(x_{1}^{\perp}, 1\right)^{\perp}\right) \wedge g_{*}\left(\left(1, x_{2}^{\perp}\right)^{\perp}\right) \\
& =g_{*}\left(x_{1}, 0\right) \wedge g_{*}\left(0, x_{2}\right) \\
& =g_{*}\left(\left(x_{1}, 0\right) \vee\left(0, x_{2}\right)\right)=g_{*}\left(x_{1}, x_{2}\right) .
\end{aligned}
$$

4.3.12 Example The dagger biproducts of OMLatGal provide it with enrichment in commutative monoids by Theorem 2.3.18. This boils down to

$$
\begin{aligned}
& (f+g)_{*}(x)=f_{*}(x) \wedge g_{*}(x), \\
& (f+g)^{*}(y)=f^{*}(y) \wedge g^{*}(y) .
\end{aligned}
$$

The associated order, as in Proposition 4.2.11, is determined by

$$
f \leq g \Longleftrightarrow g=f+g \Longleftrightarrow g_{*} \leq f_{*} \text { and } g^{*} \leq f^{*} \text { pointwise. }
$$


Thus the category OMLatGal is enriched over join-semilattices.

4.3.13 It is unclear whether the category OMLatGal has other monoidal structures besides biproducts. Next to the disadvantages mentioned in Chapter 1. there are no suitable tensor products of orthomodular lattices such that $\operatorname{KSub}(X) \otimes \operatorname{KSub}(Y) \cong \operatorname{KSub}(X \otimes Y)$ in the category Hilb. The lack of such a tensor product is one of the main sources of criticism on programmes that try to explain the mathematical structure of quantum physics through orthomodular lattices [100, 129, 165, 177, 209].

\subsection{Quantifiers}

The previous sections mostly studied $\operatorname{KSub}(X)$ of a single object $X$ in isolation. This section studies morphisms between different kernel subobject lattices. We have already seen one such morphism: pulling back along $f: X \rightarrow Y$ gives a morphism $f^{-1}: \operatorname{KSub}(Y) \rightarrow \operatorname{KSub}(X)$ of meet-semilattices. One of the major insights of categorical logic is that quantifiers can be described as adjoints to substitution: existential quantification $\exists_{f}$ is left adjoint to $f^{-1}$, whilst universal quantification $\forall_{f}$ is right adjoint to $f^{-1}$ [131, 154]. Notice that this general prescription, when applied to our quantum setting, is of a different nature from earlier attempts at quantifiers for quantum logic [128, 183], as it concerns multiple orthomodular lattices instead of a single one.

We start by constructing $\exists_{f}$ (thus defined) from the factorisation system of a dagger kernel category, as is standard [131, Lemma A1.3.1]. Subsequently we prove that it is left adjoint to $f^{-1}$.

4.4.1 Definition For a given kernel subobject $k: K \triangleright \longrightarrow X$ and a morphism $f: X \rightarrow Y$ in a dagger kernel category, define $\exists_{f}(k)=\operatorname{Im}(f \circ k)$, using the factorisation of Theorem 3.4.17. This yields a well-defined function $\exists_{f}: \operatorname{KSub}(X) \rightarrow$ $\operatorname{KSub}(Y)$.

4.4.2 Theorem [43. Lemma 2.5] Let $f: X \rightarrow Y$ be a morphism in a dagger kernel category. The map $\exists_{f}: \operatorname{KSub}(X) \rightarrow \operatorname{KSub}(Y)$ is monotone and left-adjoint (in the category of posets) to $f^{-1}: \operatorname{KSub}(Y) \rightarrow \operatorname{KSub}(X)$. If $g: Y \rightarrow Z$ is another morphism then $\exists_{g} \circ \exists_{f}=\exists_{g \circ f}: \operatorname{KSub}(X) \rightarrow \operatorname{KSub}(Z)$. Also $\exists_{\mathrm{id}}=\mathrm{id}$.

Proof For monotonicity of $\exists_{f}$, let $k \leq l$ in $\operatorname{KSub}(X)$. First factorise $l$ and then 
$K \rightarrow \exists_{f}(l)$ to obtain the following diagram:

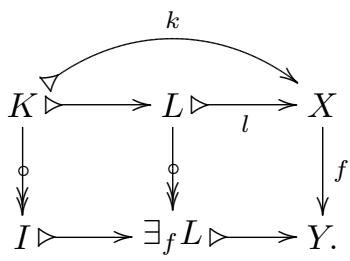

Now $K \longrightarrow \triangle \triangleright \longrightarrow Y$ is a zero epi/dagger mono factorisation of $f \circ k$, so $I$ represents $\exists_{f}(k)$, and $\exists_{f}(k) \leq \exists_{f}(l)$. To show the adjunction, let $k \in \operatorname{KSub}(X)$ and $l \in \operatorname{KSub}(Y)$, and consider the solid arrows in the following diagram:

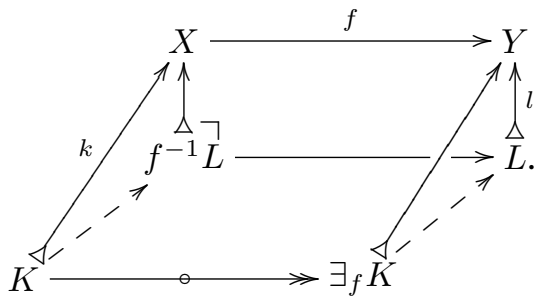

If $\exists_{f}(k) \leq l$, then the right dashed map $\exists_{f} K \rightarrow L$ exists and the outer square commutes. Hence, since $f^{-1} L$ is a pullback, the left dashed map $K \rightarrow f^{-1} L$ exists, and $k \leq f^{-1}(l)$. Conversely, if $k \leq f^{-1}(l)$, factorise the map $K \rightarrow L$ to obtain the image of $f \circ k$. In particular, this image then factors through $L$, whence $\exists_{f}(k) \leq l$. Finally, $\exists_{g} \circ \exists_{f}=\exists_{g \circ f}$ just states how left adjoints compose. $\square$

4.4.3 Proposition For $f: X \rightarrow Y$ and $k \in \mathrm{KSub}(X)$ in a dagger kernel category, one has $\exists_{f}(k)=\left(\left(f^{\dagger}\right)^{-1}\left(k^{\perp}\right)\right)^{\perp}$.

Proof One has:

$$
\begin{aligned}
\left(\left(f^{\dagger}\right)^{-1}\left(k^{\perp}\right)\right)^{\perp} \leq l & \Longleftrightarrow l^{\perp} \leq\left(f^{\dagger}\right)^{-1}\left(k^{\perp}\right) \\
& \Longleftrightarrow \text { there is a } \psi: L^{\perp} \rightarrow K^{\perp} \text { with } k^{\perp} \circ \psi=f^{\dagger} \circ l^{\perp} \\
& \Longleftrightarrow \text { there is a } \varphi: K \rightarrow L \text { with } l \circ \psi=f \circ k \\
& \Longleftrightarrow k \leq f^{-1}(l) .
\end{aligned}
$$

For the direction $(\Rightarrow)$ of the marked equivalence, recall that $l=\operatorname{ker}(\operatorname{coker}(l))$, so

$$
\operatorname{coker}(l) \circ f \circ k=\left(f^{\dagger} \circ l^{\perp}\right)^{\dagger} \circ k=\left(k^{\perp} \circ \psi\right)^{\dagger} \circ l=\psi^{\dagger} \circ \operatorname{coker}(k) \circ k=0 .
$$


The reverse direction works similarly: given $\varphi$, one obtains

$$
k^{\dagger} \circ f^{\dagger} \circ l^{\perp}=(f \circ k)^{\dagger} \circ l^{\perp}=(l \circ \varphi)^{\dagger} \circ l^{\perp}=\varphi^{\dagger} \circ l^{\dagger} \circ l^{\perp}=\varphi^{\dagger} \circ 0=0 .
$$

4.4.4 Definition A bounded lattice $X$ is called a Heyting algebra when, regarding $X$ as a category, $\left(_{-}\right) \wedge x$ has a right adjoint $x \Rightarrow\left({ }_{-}\right)$for every $x \in X$. Explicitly, a Heyting algebra $X$ comes with a monotone function $\Rightarrow: X^{\mathrm{op}} \times X \rightarrow X$ satisfying $x \leq(y \Rightarrow z)$ if and only if $x \wedge y \leq z$.

4.4.5 Lemma Suppose there are right adjoints $\forall_{f}$ to $f^{-1}: \operatorname{KSub}(Y) \rightarrow \operatorname{KSub}(X)$ for each $f: X \rightarrow Y$ in a dagger kernel category. Then each $\operatorname{KSub}(X)$ is a Heyting algebra.

Proof For $k, l \in \operatorname{KSub}(X)$, define $(k \Rightarrow l)=\forall_{k}\left(k^{-1}(l)\right)$. This is again a kernel subobject of $X$. Let $K$ be the domain of $k$. Then for any $m \in \operatorname{KSub}(X)$ :

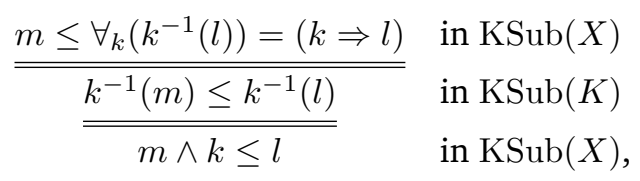

where the last equivalence follows from Lemma 4.3.2.

4.4.6 Definition A distributive lattice is a lattice satisfying the following equations:

$$
\begin{aligned}
& x \vee(y \wedge z)=(x \vee y) \wedge(x \vee z), \\
& x \wedge(y \vee z)=(x \wedge y) \vee(x \wedge z) .
\end{aligned}
$$

4.4.7 Example A Heyting algebra is necessarily a distributive lattice, because $\left(\_\right) \wedge x$, being a left adjoint, preserves colimits, i.e. joins.

For an example of a nondistributive lattice, consider the lattice $\operatorname{KSub}\left(\mathbb{C}^{2}\right)$ in the category Hilb. For the kernel subobjects represented by

$$
\begin{aligned}
& \kappa_{1}: \mathbb{C} \rightarrow \mathbb{C}^{2}, \\
& \kappa_{2}=\left(\kappa_{1}\right)^{\perp}: \mathbb{C} \rightarrow \mathbb{C}^{2}, \\
& \Delta=\langle\text { id }, \text { id }\rangle: \mathbb{C} \rightarrow \mathbb{C}^{2},
\end{aligned}
$$

we have $\kappa_{1} \wedge\left(\Delta \vee \kappa_{2}\right)=\kappa_{1} \wedge 1=\kappa_{1} \neq 0=0 \vee 0=\left(\kappa_{1} \wedge \Delta\right) \vee\left(\kappa_{1} \wedge \kappa_{2}\right)$. By the previous lemma, we conclude that there are dagger kernel categories that do not have universal quantifiers. Section 4.5 will give a wider class of dagger kernel categories that do not have universal quantifiers. 
4.4.8 In categorical logic, quantifiers usually have to satisfy an additional condition, namely the so-called Beck-Chevalley condition-if the left square below is a pullback, then the right one must commute:
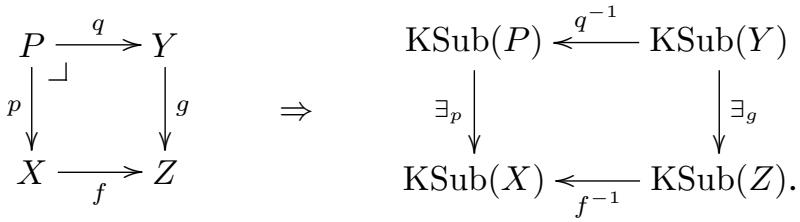

This condition ensures that $\exists$ commutes with substitution. Beck-Chevalley holds for those pullbacks from Proposition 3.4.3 that are known to exist. For a kernel $k \in \operatorname{KSub}(Y)$, we have

$$
\begin{aligned}
f^{-1}\left(\exists_{g}(k)\right) & =f^{-1}(g \circ k) \\
& =q^{-1}(k) \circ p \\
& =\exists_{p}\left(q^{-1}(k)\right) .
\end{aligned}
$$

(because both $g, k$ are kernels)

(by composition of pullbacks)

If the dagger kernel category has biproducts and equalisers, then Beck-Chevalley holds for all morphisms by [33, II, Proposition 1.7.6]. But in general, BeckChevalley cannot be expected to hold in dagger kernel categories because of Corollary 4.2.8.

Up to now, we have considered KSub to be a functor taking values in the category of meet-semilattices. Towards showing that it in fact takes values in the category OMLatGal, we now establish a connection between adjoint morphisms and adjoint functors between the corresponding kernel subobject lattices. Thus we will explicate the relationship between $\exists_{f}$ and $\exists_{f^{\dagger}}$, inspired by [174].

4.4.9 Theorem For a morphism $f: X \rightarrow Y$ in a dagger kernel category, define 2

$$
\operatorname{KSub}(f)_{*}=\left(\exists_{f}\left(\_\right)\right)^{\perp}: \operatorname{KSub}(X) \rightarrow \operatorname{KSub}(Y) .
$$

Then $\operatorname{KSub}(f)_{*}$ is left adjoint to $\operatorname{KSub}\left(f^{\dagger}\right)_{*}$.

Proof We use the alternative description of Proposition 4.4.3 for $k \in \operatorname{KSub}(X)$

\footnotetext{
${ }^{2}$ Formally, we should consider opposites of certain functors. For example, $\operatorname{KSub}(f)_{*}$ is really $\perp \circ \exists_{f}^{\text {op }}: \operatorname{KSub}(X)^{\text {op }} \rightarrow \mathrm{KSub}(Y)$ instead of $\perp \circ \exists_{f}$. We suppress them so as not to clutter our notation.
} 
and $l \in \operatorname{KSub}(Y)$ :

$$
\begin{array}{cl}
\underline{\operatorname{KSub}(f)_{*}(k)=\exists_{f}(k)^{\perp}=\left(f^{\dagger}\right)^{-1}\left(k^{\perp}\right) \leq l} & \text { in } \operatorname{KSub}(Y)^{\text {op }} \\
\frac{l \leq\left(f^{\dagger}\right)^{-1}\left(k^{\perp}\right)}{\underline{\exists_{f^{\dagger}}(l) \leq k^{\perp}}} & \text { in } \operatorname{KSub}(Y) \\
\overline{\overline{k \leq \exists_{f^{\dagger}}(l)^{\perp}=\operatorname{KSub}\left(f^{\dagger}\right)_{*}(l)}} & \text { in } \operatorname{KSub}(X) \\
\operatorname{KSub}(X) .
\end{array}
$$

In a diagram, the adjunction of the previous theorem is the following:

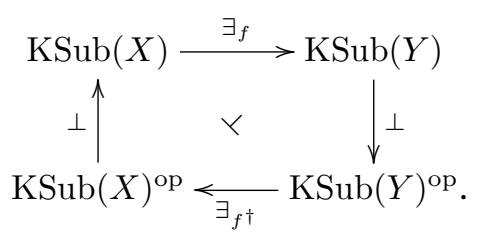

For pre-Hilbert categories whose monoidal unit is a simple generator, there is a converse to the previous theorem.

4.4.10 Lemma Let I be a simple object in a pre-Hilbert category. If $f, g: X \rightrightarrows I$ satisfy $\operatorname{ker}(f) \leq \operatorname{ker}(g)$, then $g=r \circ f$ for some $r: I \rightarrow I$, which is unique unless $f=0$.

PRoof Factorise $f$ as $f=e_{f} \circ i_{f}$ for a cokernel $e_{f}$ and a kernel $i_{f}$. Then either $i_{f}=0$ or $i_{f}=1$, since $I$ is simple. In the first case, $f=0$, whence $g=0 \circ f$. In the second case, $f$ is a cokernel, and we are left with the following situation:

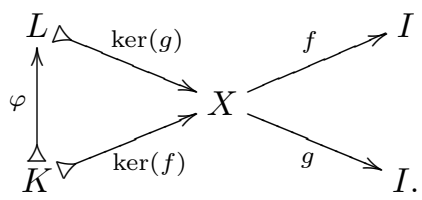

Now $f=e_{f}=\operatorname{coker}\left(\operatorname{ker}\left(e_{f}\right)\right)=\operatorname{coker}(\operatorname{ker}(f))$ by Lemma 3.4.1(c), and we have $g \circ \operatorname{ker}(f)=g \circ \operatorname{ker}(g) \circ \varphi=0$. Hence there is a unique $r$ such that $g=r \circ f$.

4.4.11 Theorem In a pre-Hilbert category whose monoidal unit $I$ is a simple generator, if $\operatorname{KSub}(f)_{*} \dashv \operatorname{KSub}(g)_{*}$, then $g=r \bullet f^{\dagger}$ for a scalar $r: I \rightarrow I$, which is unique unless $f=0$.

Proof In general, for $g: Y \rightarrow X$, the adjunction $\operatorname{KSub}(f)_{*} \dashv \operatorname{KSub}(g)_{*}$ means that for $m \in \operatorname{KSub}(X)$ and $n \in \operatorname{KSub}(Y)$, one has $n \leq \operatorname{ker}\left(\operatorname{Im}(f \circ m)^{\dagger}\right)$ if and 
only if $m \leq \operatorname{ker}\left(\operatorname{Im}(g \circ n)^{\dagger}\right)$.
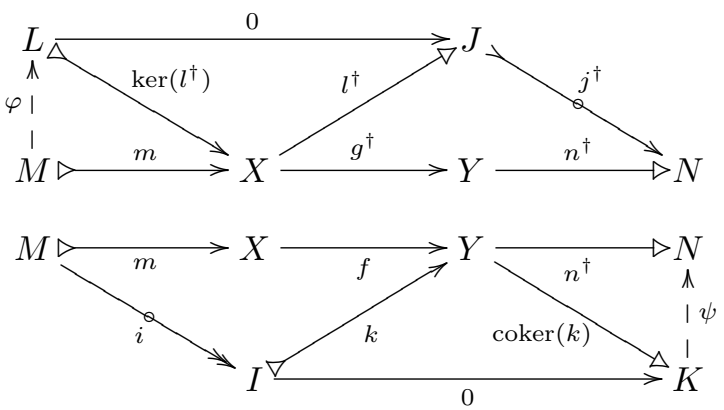

Hence the adjunction of the hypothesis means that there is a $\varphi$ making the upper diagram in (4.5) commute if and only if there is a $\psi$ making the lower one commute. So, if $n^{\dagger} \circ f \circ m=0$, then $n^{\dagger} \circ k \circ i=0$, and because $i$ is a zero epi it follows that $n^{\dagger} \circ k=0$. So $\psi$ exists, whence $\varphi$ exists, so that $n^{\dagger} \circ g^{\dagger} \circ m=$ $j^{\dagger} \circ 0 \circ \varphi=0$. Taking $m=\operatorname{ker}\left(n^{\dagger} \circ f\right)$ thus gives that $\operatorname{ker}\left(n^{\dagger} \circ f\right) \leq \operatorname{ker}\left(n^{\dagger} \circ g^{\dagger}\right)$ for all $n$. Applying Lemma 4.4.10yields that for all $n: I \rightarrow Y$, there exists $r_{n}: I \rightarrow I$ such that $n^{\dagger} \circ g^{\dagger}=r_{n} \circ n^{\dagger} \circ f$. Using 2.2.9(c), this becomes: for all $n: I \rightarrow Y$, there exists $r_{n}: I \rightarrow I$ with $g \circ n=\left(r_{n}^{\dagger} \bullet f^{\dagger}\right) \circ n$. We will show that all $r_{n}$ are in fact equal to each other (or to zero). If all $y: I \rightarrow Y$ would have $y=0$, then $Y \cong 0$, in which case $g=0 \bullet f^{\dagger}$. Otherwise, pick an $y: I \rightarrow Y$ with $y \neq 0$. There is an $r: I \rightarrow I$ with $g \circ y=\left(r^{\dagger} \bullet f^{\dagger}\right) \circ y$. Put $n^{\prime}=y \circ y^{\dagger} \circ n: I \rightarrow Y$ and $n^{\prime \prime}=\operatorname{ker}\left(y^{\dagger}\right) \circ \operatorname{coker}(y) \circ n: I \rightarrow Y$. Then

$$
\begin{aligned}
n^{\prime}+n^{\prime \prime} & =[\mathrm{id}, \mathrm{id}] \circ\left(\left(y \circ y^{\dagger} \circ n\right) \oplus\left(\operatorname{ker}\left(y^{\dagger}\right) \circ \operatorname{ker}\left(y^{\dagger}\right)^{\dagger} \circ n\right)\right) \circ\langle\mathrm{id}, \mathrm{id}\rangle \\
& =\left[y, y^{\perp}\right] \circ\left[y, y^{\perp}\right]^{\dagger} \circ n \\
& =n .
\end{aligned}
$$

Moreover,

$$
\left(r_{n^{\prime}}^{\dagger} \bullet f^{\dagger}\right) \circ n^{\prime}=g \circ n^{\prime}=g \circ y \circ y^{\dagger} \circ n=\left(r^{\dagger} \bullet f^{\dagger}\right) \circ y \circ y^{\dagger} \circ n=\left(r^{\dagger} \bullet f^{\dagger}\right) \circ n^{\prime},
$$

so $r_{n^{\prime}}=r$. Finally

$$
\begin{aligned}
\left(\left(r_{n^{\prime}}^{\dagger} \bullet f^{\dagger}\right) \circ n^{\prime}\right)+\left(\left(r_{n^{\prime \prime}}^{\dagger} \bullet f^{\dagger}\right) \circ n^{\prime \prime}\right) & =\left(g \circ n^{\prime}\right)+\left(g \circ n^{\prime \prime}\right) \\
& =g \circ n \\
& =\left(r_{n}^{\dagger} \bullet f^{\dagger}\right) \circ n \\
& =\left(\left(r_{n}^{\dagger} \bullet f^{\dagger}\right) \circ n^{\prime}\right)+\left(\left(r_{n}^{\dagger} \bullet f^{\dagger}\right) \circ n^{\prime \prime}\right) .
\end{aligned}
$$

Hence $r_{n}=r_{n^{\prime}}=r$ for all $n: I \rightarrow Y$, and we have $g \circ n=\left(r^{\dagger} \bullet f^{\dagger}\right) \circ n$. But since 
$I$ is a generator, $g=r^{\dagger} \bullet f^{\dagger}$. Reviewing our choice of $r$ in the above proof, we see that it is unique unless $f=0$.

As a consequence, we find that, modulo scalars, the passage from morphisms $f$ to functors $\operatorname{KSub}(f)_{*}$ is one-to-one. We are now ready to view KSub as a functor taking values in OMLatGal.

4.4.12 Proposition Let $\mathbf{D}$ be a dagger kernel category. Then KSub is a functor $\mathbf{D} \rightarrow$ OMLatGal. It acts on morphisms $f: X \rightarrow Y$ of $\mathbf{D}$ as

$$
\begin{aligned}
& \operatorname{KSub}(f)_{*}=\left(\exists_{f}\left(\_\right)\right)^{\perp}, \\
& \operatorname{KSub}(f)^{*}=f^{-1}\left(\left(\_\right)^{\perp}\right) .
\end{aligned}
$$

Proof On objects, we already saw that $\operatorname{KSub}(X)$ is an orthomodular lattice in Theorem 4.3.3. On morphisms, it is indeed the case that $\operatorname{KSub}(f)^{*} \dashv \operatorname{KSub}(f)_{*}$ :

$$
\begin{array}{cl}
\frac{\operatorname{KSub}(f)^{*}(n)=f^{-1}\left(n^{\perp}\right) \leq m}{\overline{m \leq f^{-1}\left(n^{\perp}\right)}} & \text { in } \operatorname{KSub}(X)^{\text {op }} \\
\overline{\overline{\exists_{f}(m) \leq n^{\perp}}} & \text { in } \operatorname{KSub}(X) \\
\overline{\overline{n \leq\left(\exists_{f}(m)\right)^{\perp}=\operatorname{KSub}(f)_{*}(m)}} & \text { in } \operatorname{KSub}(Y) \\
\operatorname{KSub}(Y) .
\end{array}
$$

We now set out to prove that the functor KSub: $\mathbf{D} \rightarrow$ OMLatGal preserves a great deal of the structure of $\mathbf{D}$.

4.4.13 Lemma In a dagger kernel category, for any $l: L \rightarrow X$ in $\operatorname{KSub}(X)$, there is an order isomorphism $\operatorname{KSub}(L) \cong \mathrm{KSub}(X)[l]=\downarrow l \subseteq \mathrm{KSub}(X)$.

Proof The direction $\operatorname{KSub}(L) \rightarrow \operatorname{KSub}(X)[l]$ of the desired bijection is given by $m \mapsto l \circ m$. This is well-defined since a composition of kernels is again a kernel. The other direction $\operatorname{KSub}(X)[l] \rightarrow \operatorname{KSub}(L)$ is $n \mapsto \varphi$, where $n=l \circ \varphi$. One easily checks that these maps are each other's inverse, and preserve the order. $\square$

4.4.14 Theorem Let $\mathbf{D}$ be an arbitrary dagger kernel category. Then the functor KSub: D $\rightarrow$ OMLatGal is a morphism of DagKerCat.

Proof By Theorem 4.4.9 we have $\operatorname{KSub}(f)_{*} \dashv \operatorname{KSub}\left(f^{\dagger}\right)_{*}$, whence $\operatorname{KSub}\left(f^{\dagger}\right)=$ $\operatorname{KSub}(f)^{\dagger}$. Preservation of the zero object is easy: $\operatorname{KSub}(0)=\{0\}=\underline{0}$. Next, let $l: L \rightarrow X$ be the kernel of a morphism $f: X \rightarrow Y$ in D. By Corollary 4.2.8, $l$ can be described as inverse image $l=f^{-1}(0)=f^{-1}\left(1^{\perp}\right)=\operatorname{KSub}(f)^{*}(1)$. Hence Lemma 4.4.13 and Lemma 4.3.8 yield the isomorphism on the left in the 
following diagram:

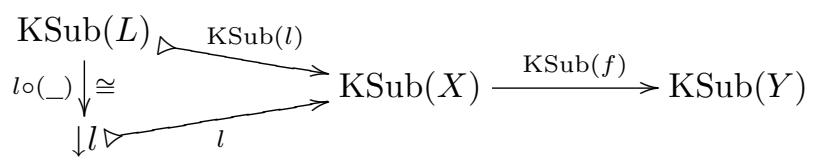

It yields a commuting triangle since for $n \in \operatorname{KSub}(K)$,

$$
\operatorname{KSub}(l)_{*}(n)=\exists_{l}(n)^{\perp}=\operatorname{Im}(l \circ n)^{\perp}=(l \circ n)^{\perp}=l_{*}(l \circ n) .
$$

Similarly for $m \in \operatorname{KSub}(X)$,

$$
l \circ \operatorname{KSub}(l)^{*}(m)=l \circ l^{-1}\left(m^{\perp}\right)=l \wedge m^{\perp}=l^{*}(m) .
$$

4.4.15 Example 4.4.7 showed that the orthomodular lattices $\operatorname{KSub}(X)$ in a dagger kernel category do not have a Heyting implication $\Rightarrow$, in general. The best possible approximation is the Sasaki hook $\Rightarrow_{\mathrm{S}}[62,82]$. This is defined by

$$
x \Rightarrow_{\mathrm{S}} y=x^{\perp} \vee(x \wedge y),
$$

and satisfies $x \wedge y \leq z$ iff $x \leq y \Rightarrow_{\mathrm{S}} z$ for $y$ and $z$ that are compatible, in the sense that $y=\left(y \wedge z^{\perp}\right) \vee(y \wedge z)$.

The following proposition shows that the Sasaki hook arises naturally in our setting of dagger kernel categories. Thus it makes the dynamical aspects of quantum logic described in [54] explicit.

4.4.16 Proposition For kernels $m: M \triangleright \longrightarrow X, n: N \triangleright \longrightarrow X$ in a dagger kernel category, the pullback $P(m)^{-1}(n)$ is the Sasaki hook:

$$
m \Rightarrow_{\mathrm{S}} n=P(m)^{-1}(n) .
$$

The associated left adjoint $\exists_{P(m)} \dashv P(m)^{-1}$ yields the and then operator $k \& m$ defined as $\exists_{P(m)}(k)=m \wedge\left(m^{\perp} \vee k\right)$, so that the following "Sasaki adjunction" holds by construction: $k \& m \leq n \Longleftrightarrow k \leq m \Rightarrow_{\mathrm{S}} n$.

PROOF Consider the following pullbacks:
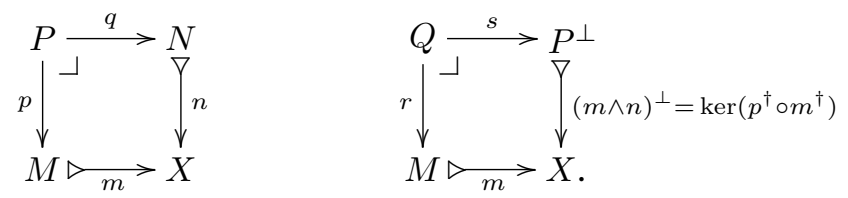
Then:

$$
\begin{aligned}
m^{\perp} \vee(m \wedge n) & =\left(m \wedge(m \wedge n)^{\perp}\right)^{\perp} \\
& =\operatorname{ker}\left(\left(m \wedge(m \wedge n)^{\perp}\right)^{\dagger}\right) \\
& =\operatorname{ker}\left(r^{\dagger} \circ m^{\dagger}\right) \\
& =\operatorname{ker}\left(\operatorname{ker}\left(\operatorname{coker}\left((m \wedge n)^{\perp}\right) \circ m\right)^{\dagger} \circ m^{\dagger}\right) \quad \text { (by Proposition 3.4.3. } \\
& =\operatorname{ker}\left(\operatorname{ker}\left(\operatorname{coker}\left(\operatorname{ker}\left(p^{\dagger} \circ m^{\dagger}\right)\right) \circ m\right)^{\dagger} \circ m^{\dagger}\right) \\
& =\operatorname{ker}\left(\operatorname{ker}\left(p^{\dagger} \circ m^{\dagger} \circ m\right)^{\dagger} \circ m^{\dagger}\right) \\
& =\operatorname{ker}\left(\operatorname{coker}(p) \circ m^{\dagger}\right) \\
& =\left(m^{\dagger}\right)^{-1}(p) \\
& =\left(m^{\dagger}\right)^{-1}\left(m^{-1}(n)\right) \\
& =P\left(m^{-1}(n) .\right.
\end{aligned}
$$

4.4.17 Quantum logic based on the and then connective \& is developed in [156], see also [184, 185]. This \& connective is in general noncommutative and non-associative. (In particular, the and then connective \& should not be confused with the multiplication of a quantale, since the latter is always associative.) Some basic properties, which easily follow from the Sasaki adjuntion, are:

- $m \& m=m$;

- $1 \& m=m \& 1=m$;

- $0 \& m=m \& 0=0$;

- if $k \& m \leq n$ and $k^{\perp} \& m \leq n$, then $m \leq n$.

The noncommutativity of $\&$ in general indicates that categorical logic in dagger kernel categories is temporal, in the sense that the order of asserting predicates matters.

4.4.18 Example The pullback $R^{-1}(l)$ of a kernel $l=(L=L \longmapsto Y)$ along a relation $R \subseteq X \times Y$, as described in Proposition 4.4.16, is the subset of $X$ given by the modal formula

$$
\square_{R}(l)(x)=R^{-1}(l)(x) \Longleftrightarrow\left(\forall_{y \in Y} \cdot(x, y) \in R \Rightarrow y \in L\right) .
$$

As is well known in modal logic, $\square_{R}$ preserves conjunctions, but not disjunctions. This again exhibits the dynamic character of the categorical logic of dagger kernel categories. 
The functor KSub: $\mathbf{D} \rightarrow$ OMLatGal can be seen as an indexed category, and is therefore essentially the same thing as a fibration via the Grothendieck construction that we used before in 2.4.7; see [125, Section 1.10] or [4]. We end this section with a discussion of 'fibred' properties of the functor KSub, but instead of (op)fibrations, we only consider (op)indexed posets for simplicity.

4.4.19 Definition An indexed poset is a functor $F: \mathbf{C}^{\text {op }} \rightarrow$ POSet to the category of partially ordered sets and monotone functions. These form a very simple instance of categorical logic. Intuitively, regarding the objects $X$ of the index category $\mathbf{C}$ as types, $F(X)$ are predicates (with free variable ranging) over that type $X$. Quantifiers then boil down to $\exists_{f} \dashv F f \dashv \forall_{f}$ for morphisms $f$ of $\mathbf{C}$.

A morphism of indexed posets from $F: \mathbf{B}^{\mathrm{op}} \rightarrow$ POSet to $G$ : $\mathbf{C}^{\mathrm{op}} \rightarrow$ POSet is a functor $H: \mathbf{B} \rightarrow \mathbf{C}$ for which there is a natural transformation $F \Rightarrow G \circ H^{\mathrm{op}}$. A morphism $H$ of indexed posets is called a change-of-base when the natural transformation is a natural isomorphism. Indexed posets and their morphisms form a category IPOSet.

Similarly, an op-indexed poset if a functor $F: \mathbf{C} \rightarrow \mathbf{P O S e t}$, and a morphism of op-indexed posets $F \rightarrow G$ is a functor $H$ for which there is a natural transformation $F \Rightarrow G \circ H$. The category of op-indexed posets is denoted by oIPOSet.

4.4.20 Proposition There is a functor DagKerCat $\rightarrow$ IPOSet that acts on an object $\mathbf{D}$ as the functor $\mathrm{KSub}^{*}$ determined by $X \mapsto \operatorname{KSub}(X)$ and $f \mapsto \mathrm{KSub}(f)^{*}$.

There is also a functor DagKerCat $\rightarrow$ oIPOSet that acts on an object $\mathbf{D}$ as the functor $\mathrm{KSub}_{*}$ determined by $X \mapsto \operatorname{KSub}(X)$ and $f \mapsto \operatorname{KSub}(f)_{*}$.

Proof A morphism $F: \mathbf{D} \rightarrow \mathbf{E}$ of DagKerCat induces a natural transformation $\mathrm{KSub}^{*} \Rightarrow \mathrm{KSub}^{*} \circ F^{\mathrm{op}}$, since for $f: X \rightarrow Y$ in $\mathbf{D}$ and $l \in \operatorname{KSub}^{*}(Y)$,

$$
\begin{aligned}
\left(F \circ \mathrm{KSub}^{*}(f)\right)(l) & =F\left(f^{-1}\left(l^{\perp}\right)\right) \\
& =(F f)^{-1}\left((F l)^{\perp}\right) \\
& =(F f)^{-1}\left(F\left(l^{\perp}\right)\right)=\left(\operatorname{KSub}^{*}(f) \circ F\right)(l),
\end{aligned}
$$

because $F$ preserves kernels and daggers. Similarly, $F$ induces a natural transformation $\mathrm{KSub}_{*} \Rightarrow \mathrm{KSub}_{*} \circ F$, since

$$
\begin{aligned}
\left(F \circ \mathrm{KSub}_{*}(f)\right)(l) & =F\left(\left(\exists_{f}(l)\right)^{\perp}\right) \\
& =F\left(\left(f^{\dagger}\right)^{-1}\left(l^{\perp}\right)\right) \\
& =\left((F f)^{\dagger}\right)^{-1}\left((F l)^{\perp}\right) \\
& =\left(\exists_{F f}(F l)\right)^{\perp}=\left(\operatorname{KSub}_{*}(f) \circ F\right)(l),
\end{aligned}
$$

where we used the alternative description of $\exists$ of Proposition 4.4.3. 
4.4.21 Example The contravariant powerset functor induces an indexed poset $\mathcal{P}:$ Set $^{\mathrm{op}} \rightarrow$ POSet. The graph functor $\mathcal{G}:$ Set $\rightarrow$ Rel now induces a morphism $\mathcal{P} \rightarrow \mathrm{KSub}_{\mathrm{Rel}}$ of indexed categories, since for $f: X \rightarrow Y$ in Set and $L \subseteq Y$ :

$$
\begin{aligned}
\mathcal{G}(f)^{-1}(L) & =\left\{x \in X \mid \forall_{y \in Y} \cdot(x, y) \in \mathcal{G}(f) \Rightarrow y \in L\right\} \\
& =\left\{x \in X \mid \forall_{y \in Y} . f(x)=y \Rightarrow y \in L\right\} \\
& =\{x \in X \mid f(x) \in L\} \\
& =f^{-1}(L) .
\end{aligned}
$$

In fact, the graph functor induces a change-of-base, as $\mathcal{P}(X) \cong \mathrm{KSub}_{\mathbf{R e l}}(\mathcal{G}(X))$ by Example 4.1.4. Hence the familiar categorical logic of Set can be obtained from the logic of the dagger kernel category Rel. (See also [111].)

The graph functor is also a morphism of op-indexed posets: for $f: X \rightarrow Y$ and $K \subseteq X$ in Set, one has

$$
\begin{aligned}
\exists_{\mathcal{G}}(f)(K) & =\left\{y \in Y \mid \exists_{x \in X} \cdot(x, y) \in \mathcal{G}(f) \wedge x \in K\right\} \\
& =\left\{y \in Y \mid \exists_{x \in X} \cdot f(x)=y \wedge x \in K\right\} \\
& =\{f(x) \mid x \in K\}=\exists_{f}(K),
\end{aligned}
$$

where $\exists_{f}$ in the last line is left adjoint to pullback $f^{-1}$ in the category Set.

4.4.22 Example By 3.2.26, the functor $\ell^{2}:$ PInj $\rightarrow$ Hilb of 3.1 .13 is a morphism of DagKerCat, and hence induces a morphism of indexed posets and a morphism of op-indexed posets. It is not a change-of-base, since the map $\operatorname{KSub}_{\mathbf{P I n j}}(X)=\mathcal{P}(X) \rightarrow \operatorname{KSub}_{\mathbf{H i l b}}\left(\ell^{2}(X)\right)$ is not an isomorphism.

4.4.23 Example The functor $P:$ Hilb $\rightarrow$ PHilb of Example 2.1.4 is a morphism of DagKerCat by Example 3.2.27. Hence it induces a morphism of indexed posets, and a morphism of op-indexed posets. In fact, it induces a change-of-base, since kernels in PHilb are precisely kernels in Hilb by Example 3.2 .27 .

\subsection{Booleanness}

This section characterises precisely when the kernel subobject lattices in a dagger kernel category represent classical logics, i.e. when they are not just orthomodular lattices but Boolean algebras. Accordingly, such dagger kernel categories are called Boolean. This notion gives rise to a generic construction that generalises the way the category of partial injections can be obtained from the category of relations. We also discuss a method of turning an arbitrary Boolean algebra into a Boolean dagger kernel category. 
4.5.1 Definition A dagger kernel category is called Boolean if $k \wedge l=0$ implies $k^{\dagger} \circ l=0$, for all kernels $k, l$.

4.5.2 Lemma A dagger kernel category is Boolean if and only if

$$
k \wedge l=0 \Longleftrightarrow k \perp l
$$

for all $k, l \in \operatorname{KSub}(X)$, i.e. when kernels are disjoint if and only if they are orthogonal.

Proof Booleanness means that $k \wedge l=0$ implies $k^{\dagger} \circ l=0$, which is equivalent to $l^{\dagger} \circ k=0$, which is $k \perp l$ by definition. The reverse implication is easy, too: if $k \circ f=l \circ g$, then $f=k^{\dagger} \circ k \circ f=k^{\dagger} \circ l \circ g=0 \circ g=0$. Similarly, $g=0$. Hence the pullback of $k$ and $l$ is the zero object 0 .

4.5.3 A Boolean algebra is a Heyting algebra that is at the same time an orthomodular lattice with the same lattice operations. More precisely, a Boolean algebra is a Heyting algebra in which $x=x^{\perp \perp}$ for all $x$, where $x^{\perp}$ is defined to be $(x \Rightarrow 0)$. It is usual to denote the latter by $\neg x$ instead of $x^{\perp}$ in case the algebra is Boolean. Boolean algebras form a category BoolAlg, whose morphisms are functions that preserves finite meets and joins.

4.5.4 Theorem A dagger kernel category is Boolean if and only if $\operatorname{KSub}(X)$ is a Boolean algebra for each object $X$.

PRoOF We already know from Theorem 4.3.3 that each $\operatorname{KSub}(X)$ is an orthomodular lattice. As a distributive orthomodular lattice is Boolean, it suffices to show that distributivity $k \wedge(l \vee m)=(k \vee l) \wedge(k \vee m)$ is equivalent to the Booleanness requirement $k \wedge l=0 \Rightarrow k \perp l$.

First, assume Booleanness. Then

$$
\left(k \wedge(k \wedge l)^{\perp}\right) \wedge l=(k \wedge l) \wedge(k \wedge l)^{\perp}=0 .
$$

Hence $k \wedge(k \wedge l)^{\perp} \leq l^{\perp}$. Similarly, $k \wedge(k \wedge m)^{\perp} \leq m^{\perp}$. Therefore,

$$
k \wedge(k \wedge l)^{\perp} \wedge(k \wedge m)^{\perp} \leq l^{\perp} \wedge m^{\perp}=(l \vee m)^{\perp},
$$

whence

$$
k \wedge(k \wedge l)^{\perp} \wedge(k \wedge m)^{\perp} \wedge(l \vee m)=0 .
$$

But then we are done by using Booleanness again:

$$
k \wedge(l \vee m) \leq\left((k \wedge l)^{\perp} \wedge(k \wedge m)^{\perp}\right)^{\perp}=(k \wedge l) \vee(k \wedge m)
$$


For the converse, suppose that $k \wedge l=0$. Then

$$
\begin{aligned}
k & =k \wedge 1 \\
& =k \wedge\left(l \vee l^{\perp}\right) \\
& =(k \wedge l) \vee\left(k \wedge l^{\perp}\right) \\
& =0 \vee\left(k \wedge l^{\perp}\right)=k \wedge l^{\perp},
\end{aligned}
$$

(by distributivity)

whence $k \leq l^{\perp}$.

4.5.5 Example The previous theorem entails that Rel and PInj are Boolean dagger kernel categories, whereas Hilb, PHilb and OMLatGal are not.

It also entails that Boolean dagger kernel categories cannot have universal quantifiers, as in the following corollary, which extends Example 4.4.7.

4.5.6 Corollary A dagger kernel category that is not Boolean cannot have right adjoints $\forall_{f}$ to $f^{-1}$ for all morphisms $f$.

PROOF If the category is not Boolean, by the previous theorem there is an object $X$ such that $\operatorname{KSub}(X)$ is an orthomodular lattice that is not Boolean. That means that $\operatorname{KSub}(X)$ is not distributive, and hence cannot be a Heyting algebra. Hence the existence of universal quantifiers would contradict Lemma 4.4.5.

The Booleanness property can be strengthened in the following way.

4.5.7 Proposition The following are equivalent for a dagger kernel category D:

(a) $\mathbf{D}$ is Boolean;

(b) for each pullback of kernels

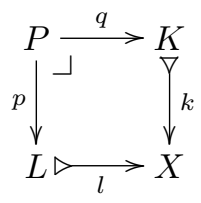

one has $l^{\dagger} \circ k=p \circ q^{\dagger}$.

Proof It is easy to see that the definition of Booleanness is the special case $P=$ 0 . To see the converse, put another pullback left to the one in the proposition:

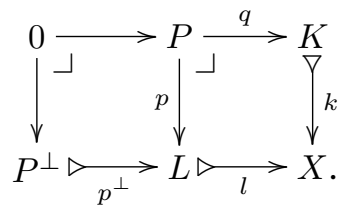


We use that $p, q$ are kernels by Proposition 3.4.3. Then $k \wedge\left(l \circ p^{\perp}\right)=0$, so by Booleanness:

$$
\begin{aligned}
k \leq\left(l \circ p^{\perp}\right)^{\perp} & =\operatorname{ker}\left(\left(l \circ \operatorname{ker}\left(p^{\dagger}\right)\right)^{\dagger}\right) \\
& =\operatorname{ker}\left(\operatorname{coker}(p) \circ l^{\dagger}\right) \\
& =\left(l^{\dagger}\right)^{-1}(p),
\end{aligned}
$$

where the pullback is as described in Proposition 3.4.3. Hence there is a map $\varphi: K \rightarrow P$ such that $p \circ \varphi=l^{\dagger} \circ k$. This means that

$$
\varphi=p^{\dagger} \circ p \circ \varphi=p^{\dagger} \circ l^{\dagger} \circ k=(l \circ p)^{\dagger} \circ k=(k \circ q)^{\dagger} \circ k=q^{\dagger} \circ k^{\dagger} \circ k=q^{\dagger} .
$$

Thus $p \circ q^{\dagger}=l^{\dagger} \circ k$, as required.

4.5.8 Proposition 4.5.7 could be understood as saying that Boolean dagger kernel categories have composition by pullback. In this sense, it is remarkable that the map $\ell^{2}:$ PInj $\rightarrow$ Hilb of 3.1 .13 manages to be a functorial at all. After all, PInj has composition by pullback, but Hilb does not. In fact, the requirement of Proposition 4.5.7(b) holds for kernels $k, l$ in Hilb if and only if $k \perp l$. Hence composition in Hilb coincides with composition by pullback only on orthogonal parts of Hilb, and the functor $\ell^{2}$ lands in such a part.

4.5.9 Example We now develop a class of examples by associating a Boolean dagger kernel category to every Boolean algebra. To start, let $B$ with $(1, \wedge)$ be a meet semi-lattice. We can turn this structure into a category, for which we use the notation $\widehat{B}$. The objects of $\widehat{B}$ are elements $x \in B$, and its morphisms $x \rightarrow y$ are elements $f \in B$ with $f \leq x, y$, i.e. $f \leq x \wedge y$. There is an identity $x: x \rightarrow x$, and composition of $f: x \rightarrow y$ and $g: y \rightarrow z$ is simply $f \wedge g: x \rightarrow z$. This $\widehat{B}$ is a dagger category with $f^{\dagger}=f$. A map $f: x \rightarrow y$ is a dagger mono if $f^{\dagger} \circ f=f \wedge f=x$. Hence dagger monos are of the form $x: x \rightarrow y$ where $x \leq y$.

The construction $B \mapsto \widehat{B}$ is functorial: a morphism $h: B \rightarrow C$ of meet semilattices yields a functor $\widehat{h}: \widehat{B} \rightarrow \widehat{C}$ by $x \mapsto h(x)$. It clearly preserves the dagger.

4.5.10 Proposition If $B$ is a Boolean algebra, then $\widehat{B}$ is a Boolean dagger kernel category, yielding a dagger functor BoolAlg $\rightarrow$ DagKerCat. Moreover, every dagger mono in $\widehat{B}$ is a kernel, and $\operatorname{KSub}_{\widehat{B}}(x)$ is the (principal) downset $\downarrow x$.

Proof The bottom element $0 \in B$ yields a zero object $0 \in \widehat{B}$, and also a zero map $0: x \rightarrow y$. For an arbitrary map $f: x \rightarrow y$ there is a kernel $\operatorname{ker}(f)=\neg f \wedge x$, which is a dagger mono $\operatorname{ker}(f): \operatorname{ker}(f) \rightarrow x$ in $\widehat{B}$. Clearly, $f \circ \operatorname{ker}(f)=f \wedge \neg f \wedge$ $x=0 \wedge x=0$. If also $g: z \rightarrow x$ satisfies $f \circ g=0$, then $g \leq x, z$ and $f \wedge g=0$. 
The latter yields $g \leq \neg f$ and therefore $g \leq \neg f \wedge x=\operatorname{ker}(f)$. Hence $g$ forms the required mediating map $g: z \rightarrow \operatorname{ker}(f)$ with $\operatorname{ker}(f) \circ g=g$.

Notice that each dagger mono $k: k \rightarrow x$, where $k \leq x$, is a kernel, namely of its cokernel $\neg k \wedge x: x \rightarrow(\neg k \wedge x)$. For two kernels $k: k \rightarrow x$ and $l: l \rightarrow x$, where $k, l \leq x$, one has $k \leq l$ as kernels if and only if $k \leq l$ in $B$. Thus $\operatorname{KSub}(x)=\downarrow x$, which is again a Boolean algebra (with negation $\neg_{x} k=\neg k \wedge x$ ). The intersection $k \wedge l$ as subobjects is the meet $k \wedge l$ in $B$. This allows us to show that $\widehat{B}$ is Boolean: if $k \wedge l=0$, then $k^{\dagger} \circ l=k \circ l=k \wedge l=0$.

4.5.11 In the category $\widehat{B}$ obtained from a Boolean algebra $B$, the factorisation of $f: x \rightarrow y$ is the composite $x \stackrel{f}{\longrightarrow} f \stackrel{f}{\longrightarrow} y$. In particular, for $k \leq x$, considered as a kernel $k: k \rightarrow x$, one has $\exists_{f}(k)=(k \wedge f:(k \wedge f) \rightarrow x)$.

We now turn to a generic construction that generalises the way the category of partial injections can be obtained from the category of relations.

4.5.12 Definition Let $\mathbf{D}$ be a Boolean dagger kernel category. We write $\mathbf{D}_{\mathrm{kck}}$ for the category with the same objects as $\mathbf{D}$, but with morphisms $X \rightarrow Y$ given by cokernel-kernel pairs $(c, k)$ of the form $\left(X \stackrel{c}{\longrightarrow} \triangleright{ }_{k}^{\longrightarrow} Y\right)$. The identity on

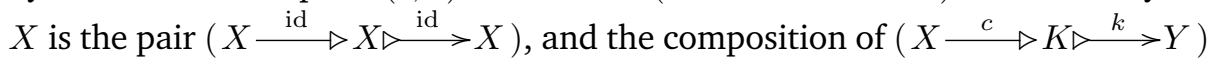
and $\left(Y \stackrel{d}{\longrightarrow} L \triangleright l \mid l Z\right.$ ) is the pair $\left(q^{\dagger} \circ c, l \circ p\right)$ obtained via the pullback

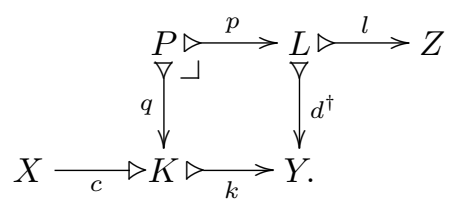

To be precise, we identify $(c, k)$ with $\left(\varphi \circ c, k \circ \varphi^{-1}\right)$, for isomorphisms $\varphi$.

Indeed, as a case in point PInj $=\mathbf{R e l}_{\mathrm{kck}}$. The $\left({ }_{-}\right)_{\mathrm{kck}}$ construction has a universal property, as the following theorem shows.

4.5.13 Theorem For a Boolean dagger kernel category $\mathbf{D}$, the category $\mathbf{D}_{\mathrm{kck}}$ is again a Boolean dagger kernel category. There is a morphism $D: \mathbf{D}_{\mathrm{kck}} \rightarrow \mathbf{D}$ in DagKerCat, and $\operatorname{KSub}_{\mathbf{D}_{\mathrm{kck}}}(X) \cong \operatorname{KSub}_{\mathbf{D}}(X)$. Moreover, in $\mathbf{D}_{\mathrm{kck}}$ one has

$$
\text { kernel }=\text { dagger mono }=\text { mono }=\text { zero mono, }
$$

and $\mathbf{D}_{\mathrm{kck}}$ is universal among Boolean dagger kernel categories $\mathbf{E}$ with a morphism cat $E_{\mathrm{kck}} \rightarrow \mathbf{D}$ and in which every zero mono is a kernel.

Proof The obvious definition $(c, k)^{\dagger}=\left(k^{\dagger}, c^{\dagger}\right)$ makes $\mathbf{D}_{\text {kck }}$ into a dagger category. The zero object $0 \in \mathbf{D}$ is also a zero object $0 \in \mathbf{D}_{\text {kck }}$ with zero map 
$X \longrightarrow 0 \triangleright \longrightarrow Y$ consisting of a cokernel-kernel pair. A morphism $(c, k)$ is a dagger mono if and only if $(c, k)^{\dagger} \circ(c, k)=\left(k^{\dagger}, k\right)$ is the identity; this means that $k=$ id.

The kernel of a morphism $(d, l)=(Y \stackrel{d}{\longrightarrow} L \triangleright \stackrel{l}{\longrightarrow} Z)$ is

$$
\operatorname{ker}(d, l)=\left(L^{\perp} \stackrel{\text { id }}{\longrightarrow} L^{\perp} \stackrel{\left(d^{\dagger}\right)^{\perp}}{\longrightarrow} Y\right),
$$

so that $\operatorname{ker}(d, l)$ is a dagger mono and $(d, l) \circ \operatorname{ker}(d, l)=0$. If also $(d, l) \circ(c, k)=0$, then $k \wedge d^{\dagger}=0$. Hence, by Booleanness, $k \leq\left(d^{\dagger}\right)^{\perp}$, say via $\varphi: K \rightarrow L^{\perp}$ with $\left(d^{\dagger}\right)^{\perp} \circ \varphi=k$. We obtain a mediating map $(c, \varphi)=\left(X \stackrel{c}{\longrightarrow} K^{\circ} \stackrel{\varphi}{\longrightarrow} L^{\perp}\right)$, which satisfies $\operatorname{ker}(d, l) \circ(c, \varphi)=\left(\mathrm{id},\left(d^{\dagger}\right)^{\perp}\right) \circ(c, \varphi)=\left(c,\left(d^{\dagger}\right)^{\perp} \circ \varphi\right)=(c, k)$. It is not hard to see that morphisms of the form (id, $k$ ) in $\mathbf{D}_{\text {kck }}$ are kernels, namely those of the cokernel $\left(k^{\perp}\right.$, id $)$. Thus $\mathbf{D}_{\text {kck }}$ is a dagger kernel category.

The intersection of two kernels (id, $k)=(K=K \bowtie \stackrel{k}{\longrightarrow} X)$ and (id, $l)=$

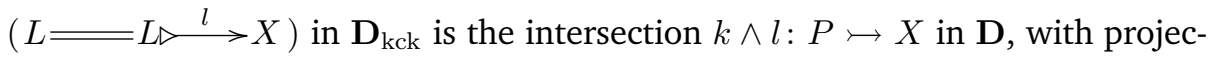
tions $(P=P \triangleright \longrightarrow K)$ and $(P=P \triangleright \longrightarrow L)$. Hence if the intersection of (id, $k$ ) and (id, $l$ ) in $\mathbf{D}_{\text {kck }}$ is 0 , then so is the intersection of $k$ and $l$ in $\mathbf{D}$, from which $l^{\dagger} \circ k=0$ follows. But then (id, $\left.l\right)^{\dagger} \circ(\mathrm{id}, k)=\left(l^{\dagger}\right.$, id $) \circ(\mathrm{id}, k)=0$ in $\mathbf{D}_{\text {kck }}$. Hence $\mathbf{D}_{\text {kck }}$ is Boolean.

There is a functor $D: \mathbf{D}_{\text {kck }} \rightarrow \mathbf{D}$ acting on objects as $D(X)=X$ and on morphisms by $D(c, k)=k \circ c$. Composition is preserved by Proposition 4.5.7. since for morphisms as in Definition 4.5.12,

$$
(d, l) \circ(c, k)=\left(q^{\dagger} \circ c, l \circ p\right) \longmapsto l \circ p \circ q^{\dagger} \circ c=(l \circ d) \circ(k \circ c) .
$$

We have already seen that kernels and dagger monos coincide. We now show that they also coincide with zero monos. So let $(d, l): Y \rightarrow Z$ be a zero-mono. This means that $(d, l) \circ(c, k)=0 \Rightarrow(c, k)=0$, for each map $(c, k)$. Using diagram (4.6), this means $d^{\dagger} \wedge k=0 \Rightarrow k=0$. By Booleanness, the antecedent $d^{\dagger} \wedge k=0$ is equivalent to $k \leq\left(d^{\dagger}\right)^{\perp}=\operatorname{ker}(d)$, which means $d \circ k=0$. Hence we see that $d$ is a zero mono in $\mathbf{D}$, and therefore is an isomorphism (because it is already a cokernel).

Finally, let $\mathbf{E}$ be a Boolean dagger kernel category in which zero monos are kernels, with a functor $F: \mathbf{E} \rightarrow \mathbf{D}$ in DagKerCat. Every morphism $f$ in $\mathbf{E}$ factors as $f=i_{f} \circ e_{f}$ for a kernel $i_{f}$ and a cokernel $e_{f}$. Hence $G: \mathbf{E} \rightarrow \mathbf{D}_{\text {kck }}$ defined by $G(X)=F(X)$ and $G(f)=\left(e_{f}, i_{f}\right)$ is the unique functor satisfying $F=D \circ G$. 


\subsection{Subobject classifiers}

To finish this chapter, we recall the notion of a subobject classifier and adapt it to our situation of kernel subobjects.

4.6.1 A subobject classifier in a category $\mathbf{C}$ with a terminal object 1 is a monomorphism $\top: 1 \rightarrow \Omega$ such that for any mono $m: M \rightarrow X$ there is a unique $\chi_{m}: X \rightarrow \Omega$ such that the following diagram is a pullback:

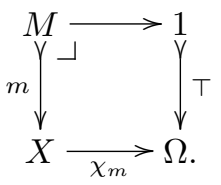

Sometimes the object $\Omega$ alone is referred to as the subobject classifier [131, A1.6]. Hence a subobject classifier $\Omega$ induces a natural isomorphism $\operatorname{Sub}(X) \cong$ $\mathbf{C}(X, \Omega)$, where the former functor acts on morphisms by pullback, the latter acts by precomposition, and the correspondence is the specific pullback $[m] \mapsto \chi_{m}$ above.

4.6.2 Example The category Set has a subobject classifier $\Omega=\{0,1\}$, with the morphism $T: 1 \rightarrow \Omega$ determined by $\top(*)=1$.

For any small category $\mathbf{C}$, the functor category $[\mathbf{C}$, Set $]$ has a subobject classifier, which we now describe. A cosieve $S$ on an object $X \in \mathbf{C}$ is a collection of morphisms with domain $X$ such that $f \in S$ implies $g \circ f \in S$ for any morphism $g$ that is composable with $f$. For $X \in \mathbf{C}$, elements of $\Omega(X)$ are the cosieves on $X$ [131, A1.6.6]. On a morphism $f: X \rightarrow Y$, the action $\Omega(f): \Omega(X) \rightarrow \Omega(Y)$ is given by

$$
\Omega(f)(S)=\{g: Y \rightarrow Z \mid Z \in \mathbf{C}, g \circ f \in S\} .
$$

Moreover, one has $F \in \operatorname{Sub}(G)$ for functors $F, G: \mathbf{C} \rightrightarrows$ Set if and only $F$ is a subfunctor of $G$, in that $F(X) \subseteq G(X)$ for all $X \in \mathbf{C}$.

In the especially easy case that $\mathbf{C}$ is a partially ordered set, seen as a category, a cosieve $S$ on $X$ is just an upper set above $X$, in the sense that $Y \in S$ and $Y \leq Z$ imply $Z \in S$ and $X \leq Y$.

To adapt this notion to our setting of kernel subobjects, we first study the object $\{0,1\}$ in the category OMLatGal. We start by explicitly describing the factorisation that results from OMLatGal being a dagger kernel category, as in Theorem 3.4.17. 
4.6.3 Lemma Let $f: X \rightarrow Y$ be an arbitrary morphism in OMLatGal. Then:

$$
\begin{aligned}
& \operatorname{coker}(f)=\left(Y \stackrel{c}{\longrightarrow} \downarrow f_{*}(1)\right), \quad \quad c_{*}(y)=y^{\perp} \wedge f_{*}(1), \\
& c^{*}(v)=v^{\perp}, \\
& \operatorname{Im}(f)=\left(\downarrow\left(f_{*}(1)^{\perp}\right) \stackrel{i_{f}}{\longrightarrow} Y\right), \quad\left(i_{f}\right)_{*}(v)=v^{\perp}, \\
& \left(i_{f}\right)^{*}(y)=y^{\perp} \wedge f_{*}(1)^{\perp}, \\
& \left(X \stackrel{e_{f}}{0} \longrightarrow \downarrow f_{*}(1)^{\perp}\right), \quad\left(e_{f}\right)_{*}(x)=f_{*}(x) \wedge f_{*}(1)^{\perp}, \\
& \left(e_{f}\right)^{*}(v)=f^{*}(v) \text {, } \\
& \left(\downarrow f^{*}(1)>\stackrel{m_{f}}{0} \gg \downarrow f_{*}(1)^{\perp}\right), \quad\left(m_{f}\right)_{*}(x)=f_{*}(x) \wedge f_{*}(1)^{\perp}, \\
& \left(m_{f}\right)^{*}(v)=f^{*}(v) \wedge f^{*}(1)^{\perp} \text {. }
\end{aligned}
$$

Moreover, $f$ is zero epi if and only if $f_{*}(1)=0$.

PROOF This is just a matter of unravelling definitions. For instance,

$$
\begin{aligned}
& \operatorname{coker}(f)=\operatorname{ker}\left(f^{\dagger}\right)^{\dagger}=\left(\downarrow\left(f^{\dagger}\right)^{*}(1) \triangleright \longrightarrow Y\right)^{\dagger}=\left(Y \longrightarrow \downarrow f_{*}(1)\right), \\
& \operatorname{Im}(f)=\operatorname{ker}(\operatorname{coker}(f))=\operatorname{ker}\left(Y \longrightarrow f_{*}(1)\right)=\left(\downarrow f_{*}(1)^{\perp} \triangleright \longrightarrow\right) .
\end{aligned}
$$

Hence $f$ is zero epi, which by definition means $\operatorname{coker}(f)=0$, if and only if $f_{*}(1)=0$. We check that $i_{f} \circ e_{f}=f$, as required.

$$
\left(i_{f} \circ e_{f}\right)_{*}(x)=\left(\left(e_{f}\right)_{*}(x)^{\perp} \wedge f_{*}(1)^{\perp}\right)^{\perp}=\left(f_{*}(x) \wedge f_{*}(1)^{\perp}\right) \vee f_{*}(1)=f_{*}(x),
$$

by orthomodularity, using that $f_{*}(1) \leq f_{*}(x)$, since $x \leq 1$. The morphism $e_{f}$ is indeed zero epic since $\left(e_{f}\right)_{*}(1)=f_{*}(1) \wedge f_{*}(1)^{\perp}=0$.

Next we first observe:

$$
f_{*}\left(x \vee f^{*}(1)\right)=f_{*}(x) \wedge f_{*}\left(f^{*}(1)\right)=f_{*}(x) \wedge 1=f_{*}(x),
$$

since $1 \leq f_{*}\left(f^{*}(1)\right)$. We use this twice below:

$$
\begin{array}{rlr}
\left(m_{f} \circ\left(i_{f^{\dagger}}\right)^{\dagger}\right)_{*}(x) & =\left(m_{f}\right)_{*}\left(f^{*}(1)^{\perp} \wedge\left(\left(f^{\dagger}\right)_{*}(1)^{\perp} \wedge x^{\perp}\right)^{\perp}\right) \\
& =f_{*}\left(f^{*}(1)^{\perp} \wedge\left(f^{*}(1) \vee x\right) \wedge f_{*}(1)^{\perp}\right. \\
& =f_{*}\left(f^{*}(1) \vee\left(f^{*}(1)^{\perp} \wedge\left(f^{*}(1) \vee x\right)\right) \wedge f_{*}(1)^{\perp} \quad\right. \text { (by [4.7) } \\
& =f_{*}\left(f^{*}(1) \vee x\right) \wedge f_{*}(1)^{\perp} \\
& =f_{*}(x) \wedge f_{*}(1)^{\perp} \\
& =\left(e_{f}\right)_{*}(x) .
\end{array}
$$


The morphism $m_{f}$ is a zero epi:

$$
\begin{aligned}
\left(m_{f}\right)_{*}(1) & \left.=f_{*}\left(f^{*}(1)^{\perp}\right) \wedge f_{*}(1)^{\perp} \quad \text { (since } 1 \text { on the left is the greatest element of } \downarrow f^{*}(1)^{\perp}\right) \\
& \left.=f_{*}\left(f^{*}(1) \vee f^{*}(1)^{\perp}\right) \wedge f_{*}(1)^{\perp} \quad \text { (by (4.7) }\right) \\
& =f_{*}(1) \wedge f_{*}(1)^{\perp}=0 .
\end{aligned}
$$

Similarly, one shows that $m_{f}$ is a zero mono.

4.6.4 Lemma Let $f: X \rightarrow Y$ be a morphism in OMLatGal. Its inverse and direct images are given explicitly by:

$$
\begin{array}{ll}
\operatorname{KSub}(Y) \stackrel{f^{-1}}{\longrightarrow} \operatorname{KSub}(X) & \operatorname{KSub}(X) \stackrel{\exists_{f}}{\longrightarrow} \operatorname{KSub}(Y) \\
(\downarrow b \rightarrow Y) \longmapsto\left(\downarrow f^{*}\left(b^{\perp}\right) \rightarrow X\right) & (\downarrow a \rightarrow X) \longmapsto\left(\downarrow\left(f_{*}(a)^{\perp}\right) \rightarrow Y\right) .
\end{array}
$$

Proof For $b \in Y$, we have by Proposition 3.4.3 and Lemma 4.6.3.

$$
\begin{aligned}
f^{-1}(\downarrow b \rightarrow Y) & =\operatorname{ker}(\operatorname{coker}(\downarrow b \rightarrow Y) \circ f) \\
& =\operatorname{ker}((Y \rightarrow \downarrow c) \circ f) \quad\left(\text { for } c=b_{*}\left(1_{\downarrow b}\right)=\left(1_{\downarrow b}\right)^{\perp}=b^{\perp}\right) \\
& =\downarrow a \rightarrow X=\downarrow f^{*}\left(b^{\perp}\right) \rightarrow X,
\end{aligned}
$$

where

$$
a=\left(c^{\dagger} \circ f\right)^{*}\left(1_{\downarrow b}\right)=f^{*}\left(c_{*}\left(1_{\downarrow c}\right)^{\perp}\right)=f^{*}\left(c^{\perp \perp}\right)=f^{*}(c)=f^{*}\left(b^{\perp}\right) .
$$

The description of the direct image follows from Lemma 4.6.3.

$$
\exists_{f}(\downarrow a \rightarrow X)=\operatorname{Im}(f \circ(\downarrow a \rightarrow X))=\downarrow b \rightarrow Y=\downarrow\left(f_{*}(a)^{\perp}\right) \rightarrow Y,
$$

where

$$
b=(f \circ a)_{*}\left(1_{\downarrow a}\right)^{\perp}=f_{*}\left(a_{*}\left(1_{\downarrow}\right)^{\perp}\right)^{\perp}=f_{*}\left(a^{\perp \perp}\right)^{\perp} .
$$

The following proposition fulfills our promise after Lemma 4.3.8 that the principal downsets in an orthomodular lattice are precisely the dagger kernels in the category OMLatGal.

4.6.5 Proposition In OMLatGal, every dagger mono of the form $a: \downarrow a \rightarrow X$ of Lemma 4.3.8, for $a \in X$, is a kernel. This yields an isomorphism

$$
\begin{aligned}
& X \stackrel{\text { }}{X} \operatorname{KSub}(X) \\
& a \longmapsto(a: \downarrow a \rightarrow X)
\end{aligned}
$$

of orthomodular lattices that is natural, in the sense that for $f: X \rightarrow Y$ in 
OMLatGal the following diagram commutes:

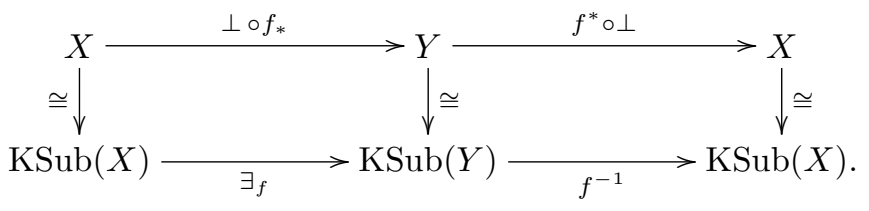

Proof We first check that $a: \downarrow a \rightarrow X$ is indeed a kernel, namely of its cokernel $\operatorname{coker}(a): X \rightarrow \downarrow a_{*}(1)$, see Lemma 4.6.3, where $a_{*}(1)=a_{*}\left(1_{\downarrow a}\right)=a_{*}(a)=a^{\perp}$. So $\operatorname{ker}(\operatorname{coker}(a))=\operatorname{coker}(a)^{*}(1)=\operatorname{coker}(a)^{*}\left(1_{\downarrow a^{\perp}}\right)=\operatorname{coker}(a)^{*}\left(a^{\perp}\right)=a^{\perp \perp}=a$.

Proposition 4.3.10 says that the mapping $X \rightarrow \operatorname{KSub}(X)$ is surjective. We now show that it is an injective homomorphism of orthomodular lattices reflecting the order, so that it is an isomorphism of orthomodular lattices.

Assume that $a \leq b$ in $X$. We can define $\varphi: \downarrow a \rightarrow \downarrow b$ by $\varphi_{*}(x)=x^{\perp_{b}}=b \wedge x^{\perp}$ and $\varphi^{*}(y)=a \wedge y^{\perp}$, for $x \in \downarrow a$ and $y \in \downarrow b$. Then $y \leq \varphi_{*}(x)$ iff $x \leq \varphi^{*}(y)$, so that $\varphi$ is a morphism in OMLatGal. In order to show $a \leq b$ in $\operatorname{KSub}(X)$ we prove $b \circ \varphi=a$. First, for $x \in \downarrow a$,

$$
\begin{aligned}
(b \circ \varphi)_{*}(x) & =b_{*}\left(\varphi_{*}(x)^{\perp_{b}}\right) \\
& =b_{*}\left(x^{\perp_{b} \perp_{b}}\right) \\
& =b_{*}(x) \\
& =x^{\perp} \\
& =a_{*}(x) .
\end{aligned}
$$

The map $X \rightarrow \operatorname{KSub}(X)$ does not only preserve the order, but also reflects it: if we have an arbitrary $\psi: \downarrow a \rightarrow \downarrow b$ with $b \circ \psi=a$, then:

$$
\begin{aligned}
a=a^{\perp \perp}=a_{*}(a)^{\perp} & =(b \circ \psi)_{*}(a)^{\perp} \\
& =b_{*}\left(\psi_{*}(a)^{\perp_{b}}\right)^{\perp} \\
& =\psi_{*}(a)^{\perp_{b} \perp \perp} \\
& =\psi_{*}(a)^{\perp_{b}} \\
& =b \wedge \psi_{*}(a)^{\perp} \\
& \leq b .
\end{aligned}
$$

This map also preserves $\perp$, since

$$
(\downarrow a \stackrel{a}{\longrightarrow} X)^{\perp}=\operatorname{ker}\left(a^{\dagger}\right)=(\downarrow b \longmapsto \stackrel{b}{\longrightarrow} X),
$$


where, according to Proposition 4.3.10,

$$
b=\left(a^{\dagger}\right)^{*}(1)=a_{*}(1)=1^{\perp}=a^{\perp},
$$

since $a$ is the greatest element in $\downarrow a$.

It remains to show that the mapping $X \rightarrow \operatorname{KSub}(X)$ preserves finite meets. It is almost immediate that it sends the top element $1 \in X$ to the identity map (top) in $\operatorname{KSub}(X)$. It also preserves finite conjunctions, since the intersection of the kernels $\downarrow a \rightarrow X$ and $\downarrow b \rightarrow X$ is given by $\downarrow(a \wedge b) \rightarrow X$. Since $a \wedge b \leq a, b$ there are appropriate maps $\downarrow(a \wedge b) \rightarrow \downarrow a$ and $\downarrow(a \wedge b) \rightarrow \downarrow b$. Suppose that we have maps $k \rightarrow \downarrow a$ and $k \rightarrow \downarrow b$, where $k: \downarrow f^{*}(1) \rightarrow X$ is a kernel of $f: X \rightarrow Y$. Since, as we have seen, the order is reflected, we obtain $f^{*}(1) \leq a, b$, and thus $f^{*}(1) \leq a \wedge b$, yielding the required map $\downarrow f^{*}(1) \rightarrow \downarrow(a \wedge b)$.

Finally, the naturality follows from Lemma 4.6.4.

4.6.6 In OMLatGal, the adjunction $\exists_{f} \dashv f^{-1}$ that exists in arbitrary dagger kernel categories boils down to the adjunction between $f^{*} \dashv f_{*}$ in the definition of morphisms in OMLatGal:

$$
\begin{aligned}
\exists_{f}(\downarrow a \rightarrow X) \leq(\downarrow b \rightarrow Y) & \Longleftrightarrow\left(\downarrow f_{*}(a)^{\perp} \rightarrow Y\right) \leq(\downarrow b \rightarrow Y) \\
& \Longleftrightarrow f_{*}(a)^{\perp} \leq b \\
& \Longleftrightarrow b^{\perp} \leq f_{*}(a) \\
& \Longleftrightarrow a \leq f^{*}\left(b^{\perp}\right) \\
& \Longleftrightarrow(\downarrow a \rightarrow X) \leq\left(\downarrow f^{*}\left(b^{\perp}\right) \rightarrow X\right) \\
& \Longleftrightarrow(\downarrow a \rightarrow X) \leq f^{-1}(\downarrow b \rightarrow X) .
\end{aligned}
$$

4.6.7 In OMLatGal, the Sasaki hook $\Rightarrow_{\mathrm{S}}$ and the and then operator \& of Proposition 4.4.16 amount to their usual definitions in the theory of orthomodular lattices [82, 136]. In the category OMLatGal, the projection $P(m)$ of Proposition 4.1.12 becomes $P(\downarrow a \rightarrow X)=a \circ a^{\dagger}: X \rightarrow X$. One finds:

$$
(\downarrow a \rightarrow X) \Rightarrow_{\mathrm{S}}(\downarrow b \rightarrow X)=P(\downarrow a \rightarrow X)^{-1}(\downarrow b \rightarrow X)=(\downarrow c \rightarrow X),
$$

where, according to Lemma 4.6 .4 ,

$$
c=\left(a \circ a^{\dagger}\right)^{*}\left(b^{\perp}\right)=a_{*}\left(a^{*}\left(b^{\perp}\right)^{\perp_{a}}\right)=\left(a \wedge(a \wedge b)^{\perp}\right)^{\perp}=a^{\perp} \vee(a \wedge b)=a \Rightarrow_{\mathrm{S}} b .
$$

Similarly for the and then operator \&:

$$
(\downarrow a \rightarrow X) \&(\downarrow b \rightarrow X)=\exists_{P(\downarrow b \rightarrow X)}(\downarrow a \rightarrow X)=(\downarrow c \rightarrow X),
$$


where the description of Lemma 4.6.4 yields:

$$
c=\left(b \circ b^{\dagger}\right)_{*}(a)^{\perp}=b_{*}\left(b^{*}(a)^{\perp_{a}}\right)^{\perp}=\left(b \wedge\left(b \wedge a^{\perp}\right)^{\perp}\right)^{\perp \perp}=b \wedge\left(b^{\perp} \vee a\right)=a \& b .
$$

4.6.8 One can define a weakest precondition modality $[f]$ from dynamic logic [14, 15, 68] in the setting of the previous example. For a morphism $f: X \rightarrow Y$ in OMLatGal and $y \in Y$, define

$$
[f](y)=f^{*}\left(y^{\perp}\right)
$$

for " $y$ holds after $f$ ". This operation $[f]\left(\_\right)$preserves meets, as usual. An element $a \in X$ yields a test operation $a ?=P(a)=a \circ a^{\dagger}$. Then one can recover the Sasaki hook $a \Rightarrow_{\mathrm{S}} b$ via this modality as $[a ?] b$, and therefore recover the complement $a^{\perp}$ as $[a$ ? $] 0$. This again exhibits the temporal, dynamic character of the categorical logic of dagger kernel categories.

We are now ready to consider "kernel subobject classifiers" for dagger kernel categories. We start with the category OMLatGal.

4.6.9 Lemma Let $2=\{0,1\}$ be the 2-element Boolean algebra, considered an orthomodular lattice $2 \in \mathrm{OMLatGal}$. For each orthomodular lattice $X$, there is an isomorphism (of sets)

$$
X \stackrel{\cong}{\longrightarrow} \operatorname{OMLatGal}(2, X)
$$

that maps $a \in X$ to $\bar{a}: 2 \rightarrow X$, given by

$$
\bar{a}_{*}(w)=\left\{\begin{array}{ll}
1 & \text { if } w=0 \\
a^{\perp} & \text { if } w=1
\end{array} \quad \bar{a}^{*}(x)= \begin{cases}1 & \text { if } x \leq a^{\perp} \\
0 & \text { otherwise. }\end{cases}\right.
$$

This isomorphism is natural, as for $f: X \rightarrow Y$ the following diagram commutes:

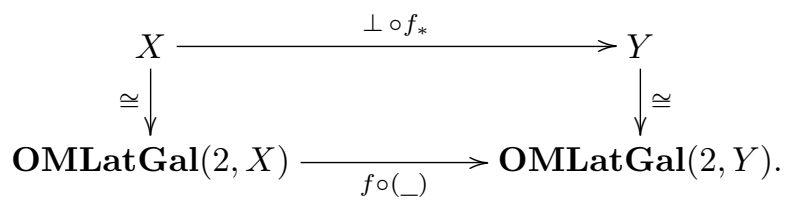

Proof The thing to note is that for a map $f: 2 \rightarrow X$ in OMLatGal we have $f_{*}(0)=1$, because $f_{*}: 2^{\text {op }} \rightarrow Y$ is a right adjoint. Hence we can only choose $a=f_{*}(1) \in X$. Once this is chosen, the left adjoint $f^{*}: X \rightarrow 2^{\text {op }}$ is completely determined, namely as $1 \leq f^{*}(x)$ iff $x \leq f_{*}(1)$. 
As for naturality, the following suffices:

$$
\begin{aligned}
(f \circ \bar{a})_{*}(1) & =f_{*}\left(\bar{a}_{*}(1)^{\perp}\right) \\
& =f_{*}\left(a^{\perp \perp}\right) \\
& =f_{*}(a)^{\perp \perp} \\
& =\bar{f}_{*\left(a^{\perp}\right)_{*}}(1) \\
& =\overline{\left(\perp \circ f_{*}\right)(a)_{*}}(1) .
\end{aligned}
$$

4.6.10 Corollary The 2-element lattice $2 \in$ OMLatGal is a "kernel subobject opclassifier". That is, there is are isomorphisms

$$
\operatorname{KSub}(X) \stackrel{\chi}{\cong} \operatorname{OMLatGal}(2, X)
$$

that are natural in that $\chi \circ \exists_{f}=f \circ \chi$ for any $f: X \rightarrow Y$.

PRoOF Of course we use the isomorphisms $\operatorname{KSub}(X) \cong X \cong$ OMLatGal from Proposition 4.6.5 and Lemma 4.6.9, so that $\chi(\downarrow a \rightarrow X)=\bar{a}$. Then, for $f: X \rightarrow$ $Y$ :

$$
\begin{aligned}
\chi\left(\exists_{f}(\downarrow a \rightarrow X)\right)_{*}(1) & =\chi\left(\downarrow\left(f_{*}(a)^{\perp}\right) \rightarrow Y\right)_{*}(1) \\
& =\overline{\left(f_{*}(a)^{\perp}\right)_{*}}(1) \\
& =f_{*}(a)^{\perp \perp} \\
& =f_{*}\left(a^{\perp \perp}\right) \\
& =f_{*}\left(\bar{a}_{*}(1)^{\perp}\right) \\
& =(f \circ \bar{a})_{*}(1) \\
& =(f \circ \chi(\downarrow a \rightarrow X))_{*}(1) .
\end{aligned}
$$

A similar phenomenon occurs in Rel, and in the Boolean dagger kernel categories $\widehat{B}$ constructed from Boolean algebras $B$ as in Definition 4.5.12.

4.6.11 Lemma The singleton set $1 \in \mathbf{R e l}$ is a "kernel subobject opclassifier". That is, there is are isomorphisms

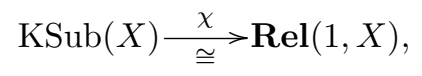

that are natural in that $\chi \circ \exists_{S}=S \circ \chi$ for any $S: X \rightarrow Y$.

PROOF In the category Rel, there are the following correspondences:

$$
\operatorname{KSub}(X) \cong \mathcal{P}(X) \cong \operatorname{Set}(X, 2) \cong \operatorname{Set}(X, \mathcal{P}(1)) \cong \operatorname{Rel}(X, 1)
$$


These induce an isomorphism $\chi$ given by

$$
\begin{aligned}
\operatorname{KSub}(X)= & \mathcal{P}(X) \stackrel{\chi}{\cong} \operatorname{Rel}(1, X) \\
(K \subseteq X) \longmapsto & \cong(*, x) \mid x \in K\} .
\end{aligned}
$$

To verify naturality, let $S: X \rightarrow Y$ in Rel. Then

$$
\begin{aligned}
S \circ \chi(K) & =\left\{(*, y) \mid \exists_{x} .(*, x) \in \chi(K) \wedge(x, y) \in S\right\} \\
& =\left\{(*, y) \mid \exists_{x} . x \in K \wedge(x, y) \in S\right\} \\
& =\left\{(*, y) \mid \exists_{S}(K)(y)\right\} \\
& =\chi\left(\exists_{S}(K)\right) .
\end{aligned}
$$

4.6.12 Lemma The greatest element 1 of a Boolean algebra B is a "kernel subobject opclassifier" for $\widehat{B}$. That is, there is are isomorphisms

$$
\operatorname{KSub}(X) \stackrel{\chi}{\cong} \widehat{B}(1, X),
$$

that are natural in that $\chi \circ \exists_{x}=x \circ \chi$ for any $x: X \rightarrow Y$.

ProOF For $x \in B$, one has

$$
\begin{aligned}
\operatorname{KSub}(x) & =\downarrow x \frac{\chi}{\cong} \\
(k \leq x) \longmapsto & \\
& \longrightarrow(k: 1 \rightarrow x)
\end{aligned}
$$

As in the proof of the previous lemma $f \circ \chi(k)=f \wedge k=\exists_{f}(k)=\chi\left(\exists_{f}(k)\right)$.

Since there is no evident "kernel subobject opclassifier" for Hilb, we refrain from casting the phenomena observed above in an official definition. 


\section{Chapter 5}

\section{Bohrification}

This final chapter studies $\mathrm{C}^{*}$-algebras, which can be considered as one-object versions of the categories studied in Chapters 2 and 3 . Commutative $\mathrm{C}^{*}$-algebras are algebras of observables for classical physics, their Gelfand spectra being the corresponding state spaces, whereas noncommutative ones model quantum physics. In noncommutative geometry, noncommutative $\mathrm{C}^{*}$-algebras are seen as generalised spaces. Locales in toposes are another generalisation of the concept of space. We introduce a technique to construct a generalised space (locale in a topos) in the latter sense from a generalised space ( $\mathrm{C}^{*}$-algebra) in the former sense, and call this procedure Bohrification, since it attemps to capture Niels Bohr's 'doctrine of classical concepts' mathematically. In the language of the logic that comes with the topos, the original $\mathrm{C}^{*}$-algebra becomes commutative, and therefore can be studied using (constructive) classical methods. Thus we establish a concept of quantum state space that carries both spatial and logical aspects. This approach was inspired by [40, 75, 76, 122, 123].

The results in this chapter were published as [45, 117-119].

\subsection{Locales and toposes}

This section introduces locales and toposes by summarising well-known results. Both are generalisations of the concept of topological space, and both also carry logical structures. We start with complete Heyting algebras. These can be made into categories in several ways. We consider a logical, an order theoretical, and a spatial perspective.

5.1.1 Definition A morphism of complete Heyting algebras is a function that preserves the operations $\wedge, \bigvee$ and $\Rightarrow$, as well as the constants 0 and 1 . We denote 
the category of complete Heyting algebras and their morphisms by CHey. This gives a logical perspective on complete Heyting algebras.

5.1.2 Definition Example 4.4.7 showed that Heyting algebras are necessarily distributive, since $\left(_{-}\right) \wedge x$ has a right adjoint and hence preserves colimits. When a Heyting algebra is complete, arbitrary joins exist, whence the following infinitary distributive law holds:

$$
\left(\bigvee_{i \in I} y_{i}\right) \wedge x=\bigvee_{i \in I}\left(y_{i} \wedge x\right)
$$

Conversely, a complete lattice that satisfies this infinitary distributive law is a Heyting algebra by defining $y \Rightarrow z=\bigvee\{x \mid x \wedge y \leq z\}$. This gives an ordertheoretical perspective on complete Heyting algebras. The category Frm of frames has complete Heyting algebras as objects; morphisms are functions that preserve finite meets and arbitrary joins. The categories Frm and CHey are not identical, because a morphism of frames does not necessarily have to preserve the Heyting implication.

5.1.3 Definition The category Loc of locales is the opposite of the category of frames. This gives a spatial perspective on complete Heyting algebras.

5.1.4 Example To see why locales provide a spatial perspective, let $X$ be a topological space. Denote its topology, i.e. the collection of open sets in $X$, by $\mathcal{O}(X)$. Ordered by inclusion, $\mathcal{O}(X)$ satisfies (5.1), and is therefore a frame. If $f: X \rightarrow Y$ is a continuous function between topological spaces, then its inverse image $f^{-1}: \mathcal{O}(Y) \rightarrow \mathcal{O}(X)$ is a morphism of frames. We can also consider $\mathcal{O}(f)=f^{-1}$ as a morphism $\mathcal{O}(X) \rightarrow \mathcal{O}(Y)$ of locales, in the same direction as the original function $f$. Thus, $\mathcal{O}\left(\_\right)$is a covariant functor from the category Top of topological spaces and continuous maps to the category Loc of locales.

5.1.5 Convention To emphasise the spatial aspect of locales, we will follow the convention that a locale is denoted by $X$, and the corresponding frame by $\mathcal{O}(X)$ (whether or not the the frame comes from a topological space) [164, 212]. Also, we will denote a morphism of locales by $f: X \rightarrow Y$, and the corresponding frame morphism by $f^{-1}: \mathcal{O}(Y) \rightarrow \mathcal{O}(X)$ (whether or not $f^{-1}$ is indeed the pullback of a function between topological spaces). A fortiori, we will write $C(X, Y)$ for $\operatorname{Loc}(X, Y)=\operatorname{Frm}(\mathcal{O}(Y), \mathcal{O}(X))$.

5.1.6 A point $x$ of a topological space $X$ may be identified with a continuous function $1 \rightarrow X$, where 1 is a singleton set with its unique topology. Extending this to locales, a point of a locale $X$ is a locale map $1 \rightarrow X$, or equivalently, a 
frame map $\mathcal{O}(X) \rightarrow \mathcal{O}(1)$. Here, $\mathcal{O}(1)=\{0,1\}=\Omega$ is the subobject classifier of Set, as detailed in Example 4.6.2.

Likewise, an open of a locale $X$ is defined as a locale morphism $X \rightarrow S$, where $S$ is the locale defined by the Sierpinski space, i.e. $\{0,1\}$ with $\{1\}$ as the only nontrivial open. The corresponding frame morphism $\mathcal{O}(S) \rightarrow \mathcal{O}(X)$ is determined by its value at 1 , so that we may consider opens in $X$ as morphisms $1 \rightarrow \mathcal{O}(X)$ in Set. If $X$ is a genuine topological space and $\mathcal{O}(X)$ its collection of opens, then each such morphism $1 \rightarrow \mathcal{O}(X)$ corresponds to an open subset of $X$ in the usual sense.

The set $\operatorname{Pt}(X)$ of points of a locale $X$ may be topologised in a natural way, by declaring its opens to be the sets of the form $\operatorname{Pt}(U)=\left\{p \in \operatorname{Pt}(X) \mid p^{-1}(U)=\right.$ 1\} for some open $U \in \mathcal{O}(X)$. This defines a functor Pt: Loc $\rightarrow$ Top [130, Theorem II.1.4]. In fact, there is an adjunction

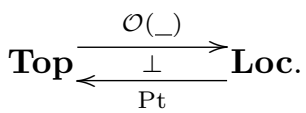

It restricts to an equivalence between so-called spatial locales and sober topological spaces. Any Hausdorff topological space is sober [130, Lemma I.1.6].

5.1.7 Example Let $(P, \leq)$ be a partially ordered set. This can be turned into a topological space by endowing it with the Alexandrov topology, in which open subsets are upper sets in $P$; principal upper sets form a basis for the topology. The associated locale $\operatorname{Alx}(P)=\mathcal{O}(P)$ thus consists of the upper sets $U P$ in $P$.

If we give a set $P$ the discrete order, then the Alexandrov topology on it is the discrete topology (in which every subset is open), and so $\mathcal{O}(P)$ is just the power set $\mathcal{P}(P)$.

As another example, we now study a way to construct frames (locales) by generators and relations. The generators form a meet-semilattice, and the relations are combined into one suitable so-called covering relation. This technique has been developed in the context of formal topology [191, 192].

5.1.8 Definition Let $L$ be a meet-semilattice. A covering relation on $L$ is a relation $\triangleleft \subseteq L \times \mathcal{P}(L)$, written as $x \triangleleft U$ when $(x, U) \in \triangleleft$, satisfying:

(a) if $x \in U$ then $x \triangleleft U$;

(b) if $x \triangleleft U$ and $U \triangleleft V$ (i.e. $y \triangleleft V$ for all $y \in U$ ) then $x \triangleleft V$;

(c) if $x \triangleleft U$ then $x \wedge y \triangleleft U$;

(d) if $x \in U$ and $x \in V$, then $x \triangleleft U \wedge V$ (where $U \wedge V=\{x \wedge y \mid x \in U, y \in V\}$ ). 
5.1.9 Example If $X \in$ Top, then $\mathcal{O}(X)$ has a covering relation defined by $U \triangleleft \mathcal{U}$ iff $U \subseteq \cup \mathcal{U}$, i.e. iff $\mathcal{U}$ covers $U$.

5.1.10 Definition Let $D L$ be the partially ordered set of all lower sets in a meetsemilattice $L$, ordered by inclusion. A covering relation $\triangleleft$ on $L$ induces a closure operation $\overline{\left(\_\right)}: D L \rightarrow D L$, namely $\bar{U}=\{x \in L \mid x \triangleleft U\}$. We define

$$
\mathcal{F}(L, \triangleleft)=\{U \in D L \mid \bar{U}=U\}=\{U \in \mathcal{P}(L) \mid x \triangleleft U \Rightarrow x \in U\} .
$$

As $\overline{\left(\_\right)}$is a closure operation, and $D L$ is a frame [130, Section 1.2], so is $\mathcal{F}(L, \triangleleft)$.

5.1.11 Proposition The frame $\mathcal{F}(L, \triangleleft)$ is the free frame on a meet-semilattice $L$ satisfying $x \leq \bigvee U$ whenever $x \triangleleft U$ for the covering relation $\triangleleft$. The canonical inclusion $i: L \rightarrow \mathcal{F}(L, \triangleleft)$, defined by $i(x)=\overline{(\downarrow x)}$, is the universal map satisfying $i(x) \leq \bigvee U$ whenever $x \triangleleft U$. That is, if $f: L \rightarrow F$ is a morphism of meetsemilattices into a frame $F$ satisfying $f(x) \leq \bigvee f(U)$ if $x \triangleleft U$, then $f$ factors uniquely through $i$.

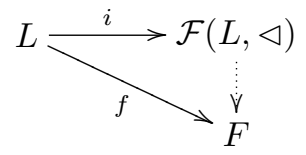

If $f$ generates $F$, in the sense that $V=\bigvee\{f(x) \mid x \in L, f(x) \leq V\}$ for each $V \in F$, there is an isomorphism of frames $F \cong \mathcal{F}(L, \triangleleft)$ where $x \triangleleft U$ iff $f(x) \leq \bigvee f(U)$.

Proof Given $f$, define $g: \mathcal{F}(L, \triangleleft) \rightarrow F$ by $g(U)=f(\bigvee U)$. For $x, y \in L$ satisfying $x \triangleleft \downarrow y$, one then has $f(x) \leq g(\bigvee \downarrow y)=f(y)$, whence $g \circ i(y)=\bigvee\{f(x) \mid$ $x \triangleleft \downarrow y\} \leq f(y)$. Conversely, $y \triangleleft \downarrow y$ because $y \in \downarrow y$, so that $f(y) \leq \bigvee\{f(x) \mid$ $x \triangleleft \downarrow y\}=g \circ i(y)$. Therefore $g \circ i=f$. Moreover, $g$ is the unique such frame morphism. The second claim is proven in [6, Theorem 12].

5.1.12 Definition Let $(L, \triangleleft)$ and $(M, \triangleleft)$ be meet-semilattices with covering relations. A continuous map $f:(M, \triangleleft) \rightarrow(L, \triangleleft)$ is a function $f^{*}: L \rightarrow \mathcal{P}(M)$ with:

(a) $f^{*}(L)=M$;

(b) $f^{*}(x) \wedge f^{*}(y) \triangleleft f^{*}(x \wedge y)$;

(c) if $x \triangleleft U$ then $f^{*}(x) \triangleleft f^{*}(U)$ (where $f^{*}(U)=\bigcup_{u \in U} f^{*}(U)$ ).

We identify two such functions if $f_{1}^{*}(x) \triangleleft f_{2}^{*}(x)$ and $f_{2}^{*}(x) \triangleleft f_{1}^{*}(x)$ for all $x \in L$.

5.1.13 Proposition Each continuous map $f:(M, \triangleleft) \rightarrow(L, \triangleleft)$ is equivalent to a frame morphism $\mathcal{F}(f): \mathcal{F}(L, \triangleleft) \rightarrow \mathcal{F}(M, \triangleleft)$ given by $\mathcal{F}(f)(U)=\overline{f^{*}(U)}$. 
5.1.14 In fact, the previous proposition extends to an equivalence $\mathcal{F}$ between the category of frames and that of formal topologies, which a generalisation of the above triples $(L, \leq, \triangleleft)$, where $\leq$ is merely required to be a preorder. In this more general case, the axioms on the covering relation $\triangleleft$ take a slightly different form. For this, including the proof of the previous proposition, we refer to [6, 24, 172].

We now generalise the concept of locales by introducing toposes.

5.1.15 Definition A topos is a category that has finite limits, exponentials (i.e. right adjoints $\left(_{-}\right)^{X}$ to $\left(\_\right) \times X$ ), and a subobject classifier (see 4.6.1).

5.1.16 Example The category Set of sets and functions is a topos: the exponential $Y^{X}$ is the set of functions $X \rightarrow Y$, and the set $\Omega=\{0,1\}$ is a subobject classifier (see Example 4.6.2).

For any small category $\mathbf{C}$, the functor category $[\mathbf{C}, \mathbf{S e t}]$ is a topos. Limits are computed pointwise [33, Theorem 2.15.2], exponentials are defined via the Yoneda embedding [164, Proposition I.6.1], and the cosieve functor $\Omega$ of Example 4.6.2 is a subobject classifier.

5.1.17 Example Without further explanation, let us mention that a sheaf over a locale $X$ is a functor from $X^{\mathrm{op}}$ (where the locale $X$ is regarded as a category via its order structure) to Set that satisfies a certain continuity condition. The category $\operatorname{Sh}(X)$ of sheaves over a locale $X$ is a topos. Its subobject classifier is $\Omega(x)=\downarrow x$ [35, Example 5.2.3].

The categories $\operatorname{Sh}(X)$ and $\operatorname{Sh}(Y)$ are equivalent if and only if the locales $X$ and $Y$ are isomorphic. Thus, toposes are generalisations of locales and hence of topological spaces. Moreover, a morphism $X \rightarrow Y$ of locales induces morphisms $\mathrm{Sh}(X) \rightarrow \operatorname{Sh}(Y)$ of a specific form: a so-called geometric morphism $\mathbf{S} \rightarrow \mathbf{T}$ between toposes is a pair of functors $f^{*}: \mathbf{T} \rightarrow \mathbf{S}$ and $f_{*}: \mathbf{S} \rightarrow \mathbf{T}$, of which $f^{*}$ preserves finite limits, with $f^{*} \dashv f_{*}$. We denote the category of toposes and geometric morphisms by Topos.

5.1.18 If $X$ is the locale resulting from putting the Alexandrov topology on a poset $P$, then $[P, \operatorname{Set}] \cong \operatorname{Sh}(X)$. In this sense Example 5.1.16 is a special case of Example 5.1.17. We call the category $[P$, Set $]$ for a poset $P$ a Kripke topos.

One could say that sheaves are the prime example of a topos in that they exhibit its spatial character as a generalisation of topology. However, this chapter is primarily concerned with functor toposes, and will therefore not mention sheaves again. We now switch to the logical aspect inherent in toposes, by sketching their internal language and its semantics. For a precise description, we refer to [131, Part D], [164, Chapter VI], or [35, Chapter 6]. 
5.1.19 In a (cocomplete) topos $\mathbf{T}$, each subobject lattice $\operatorname{Sub}(X)$ is a (complete) Heyting algebra. Moreover, pullback $f^{-1}: \operatorname{Sub}(Y) \rightarrow \operatorname{Sub}(X)$ along $f: X \rightarrow Y$ is a morphism of (complete) Heyting algebras. Finally, there are always both left and right adjoints $\exists_{f}$ and $\forall_{f}$ to $f^{-1}$. This means that we can write down properties about objects and morphisms in $\mathbf{T}$ using familiar first order logic. For example, the formula $\forall_{x \in M} \forall y \in M \cdot x \cdot y=y \cdot x$ makes sense for any object $M$ and morphism $\cdot: M \times M \rightarrow M$ in $\mathbf{T}$, and is interpreted as follows. First, the subformula $x \cdot y=y \cdot x$ is interpreted as the subobject $a: A>\longrightarrow M \times M$ given by the equaliser of $M \times M \stackrel{\cdot}{\longrightarrow} M$ and $M \times M \stackrel{\gamma}{\longrightarrow} M \times M \longrightarrow M$. Next, the subformula $\forall_{y \in M} \cdot x \cdot y=y \cdot x$ is interpreted as the subobject $b=\forall_{\pi_{1}}(a) \in$ $\operatorname{Sub}(M)$, where $\pi_{1}: M \times M \rightarrow M$. Finally, the whole formula $\forall_{x \in M} \forall_{y \in M} \cdot x \cdot y=$ $y \cdot x$ is interpreted as the subobject $c=\forall_{\pi}(b) \in \operatorname{Sub}(1)$, where $\pi: M \rightarrow 1$. The subobject $c \in \operatorname{Sub}(1)$ is classified by a unique $\chi_{c}: 1 \rightarrow \Omega$. This, then, is the truth value of the formula. In general, a formula $\varphi$ is said to hold in the topos $\mathbf{T}$, denoted by $\Vdash \varphi$, when its truth value factors through the subobject classifier $\top: 1 \rightarrow \Omega$.

If $\mathbf{T}=$ Set, the subobject $a$ is simply the set $\{(x, y) \in M \times M \mid x \cdot y=y \cdot x\}$, and therefore the truth value of the formula is $1 \in \Omega$ if for all $x, y \in M$ we have $x \cdot y=y \cdot x$, and $0 \in \Omega$ otherwise. But the above interpretation can be given in any topos $\mathbf{T}$, even if there are few or no 'elements' $1 \rightarrow M$. Thus we can often reason about objects in a topos $\mathbf{T}$ as if they were sets. Indeed, the fact that a topos has exponentials and a subobject classifier means that we can use higher order logic to describe properties of its objects, by interpreting a power set $\mathcal{P}(X)$ as the exponential $\Omega^{X}$, and the inhabitation relation $\in$ as the subobject of $X \times \Omega^{X}$ that is classified by the transpose $X \times \Omega^{X} \rightarrow \Omega$ of id: $\Omega^{X} \rightarrow \Omega^{X}$. All this can be made precise by defining the internal or Mitchell-Bénabou language of a topos, which prescribes in detail which logical formulae about the objects and morphisms of a topos are "grammatically correct" and which ones hold.

5.1.20 The interpretation of the internal language takes an especially easy form in Kripke toposes. We now give this special case of the so-called Kripke-Joyal semantics. First, let us write $\llbracket t \rrbracket$ for the interpretation of a term $t$ as in 5.1.19. For example, in the notation of 5.1.19 $\llbracket x \rrbracket$ is the morphism id: $M \rightarrow M$, and $\llbracket x \cdot y \rrbracket$ is the morphism $\cdot: M \times M \rightarrow M$. We now inductively define $p \Vdash \varphi(\vec{a})$ for $p \in P$, a formula $\varphi$ in the language of $[P$, Set $]$ with free variables $x_{i}$ of type $X_{i}$, and $\vec{a}=\left(a_{1}, \ldots, a_{n}\right)$ with $a_{i} \in X_{i}(p)$ :

- $p \Vdash\left(t=t^{\prime}\right)(\vec{a})$ if and only if $\llbracket t \rrbracket_{p}(\vec{a})=\llbracket t^{\prime} \rrbracket_{p}(\vec{a})$;

- $p \Vdash R\left(t_{1}, \ldots, t_{k}\right)(\vec{a})$ if and only if $\left(\llbracket t_{1} \rrbracket_{p}(\vec{a}), \ldots, \llbracket t_{k} \rrbracket(\vec{a})\right) \in R(p)$, where $R$ is a relation on $X_{1} \times \cdots \times X_{n}$ interpreted as a subobject of $X_{1} \times \cdots \times X_{n}$;

- $p \Vdash(\varphi \wedge \psi)(\vec{a})$ if and only if $p \Vdash \varphi(\vec{a})$ and $p \Vdash \varphi(\vec{a})$; 
- $p \Vdash(\varphi \vee \psi)(\vec{a})$ if and only if $p \Vdash \varphi(\vec{a})$ or $p \Vdash \varphi(\vec{a})$;

- $p \Vdash(\varphi \Rightarrow \psi)(\vec{a})$ if and only if $q \Vdash \varphi(\vec{a})$ implies $q \Vdash \psi(\vec{a})$ for all $q \geq p$;

- $p \Vdash \neg \varphi(\vec{a})$ if and only if $q \Vdash \varphi(\vec{a})$ for no $q \geq p$;

- $p \Vdash \exists_{x \in X} . \varphi(\vec{a})$ if and only if $p \Vdash \varphi(a, \vec{a})$ for some $a \in X(p)$;

- $p \Vdash \forall_{x \in X} \cdot \varphi(\vec{a})$ if and only if $q \Vdash \varphi(a, \vec{a})$ for all $q \geq p$ and $a \in X(q)$.

It turns out that $\varphi$ holds in $[P$, Set $]$, i.e. $\Vdash \varphi$, precisely when $p \Vdash \varphi(\vec{a})$ for all $p \in P$ and all $\vec{a} \in X_{1}(p) \times \cdots \times X_{n}(p)$.

5.1.21 The axioms of intuitionistic logic hold when interpreted in any topos, and there are toposes in whose internal language formulae that are not derivable from the axioms of intuitionistic logic do not hold. For example, the principle of excluded middle $\varphi \vee \neg \varphi$ does not hold in the topos $\operatorname{Sh}(\mathbb{R})$ [35, 6.7.2]. Thus, we can derive properties of objects of a topos as if they were sets, using the usual higher-order logic, as long as our reasoning is constructive, in the sense that we use neither the axiom of choice, nor the principle of excluded middle.

The astute reader will have noticed that the account of this chapter up to now has been constructive in this sense (including the material around Proposition 5.1.11). In particular, we can speak of objects in a topos $\mathbf{T}$ that satisfy the defining properties of locales as locales within that topos. Explicitly, these are objects $L$ that come with morphisms $0,1: 1 \rightrightarrows L$ and $\bigwedge, \bigvee: \Omega^{L} \rightrightarrows L$ for which the defining formulae of locales, such as (5.1), hold in $\mathbf{T}$ [35, Section 6.11]. The category of such objects is denoted by $\operatorname{Loc}(\mathbf{T})$, so that $\operatorname{Loc}($ Set $) \cong \operatorname{Loc}$. For the rest of this chapter we will also take care to use constructive reasoning whenever we reason in the internal language of a topos.

5.1.22 We have two ways of proving properties of objects and morphisms in toposes. First, we can take an external point of view. This occurs, for example, when we use the structure of objects in $[P$, Set $]$ as Set-valued functors. Secondly, we can adopt the internal logic of the topos, as above. In this viewpoint, we regard the topos as a 'universe of discourse'. At least intuitionistic reasoning is valid, but more logical laws might hold, depending on the topos one is studying. To end this section, we consider the internal and external points of view in several examples.

5.1.23 Example Let $\mathbf{T}$ be a topos, and $X$ an object in it. Externally, one simply looks at $\operatorname{Sub}(X)$ as a set, equipped with the structure of a Heyting algebra in the category Set. Internally, $\operatorname{Sub}(X)$ is described as the exponential $\Omega^{X}$, or $\mathcal{P}(X)$, which is a Heyting algebra object in the topos $\mathbf{T}$ [164, p. 201]. 
5.1.24 Example For any poset $P$, the category $\operatorname{Loc}([P, \mathbf{S e t}])$ is equivalent to the slice category $\operatorname{Loc} / \operatorname{Alx}(P)$ of locale morphisms $L \rightarrow \operatorname{Alx}(P)$ from some locale $L$ to the Alexandrov topology on $P$ (by 5.1.18 and [134]). Therefore, an internal locale object $\underline{L}$ in $[P$, Set $]$ is described externally as a locale morphism $f: L \rightarrow$ $\operatorname{Alx}(P)$, determined as follows. First, $\mathcal{O}(\underline{L})(P)$ is a frame in Set, and for $U$ in Alx $(P)$, the action $\mathcal{O}(\underline{L})(P) \rightarrow \mathcal{O}(\underline{L})(U)$ on morphisms is a frame morphism. Since $\mathcal{O}(\underline{L})$ is complete, there is a left adjoint $l_{U}^{-1}: \mathcal{O}(\underline{L})(U) \rightarrow \mathcal{O}(\underline{L})(P)$, which in turn defines a frame morphism $f^{-1}: \mathcal{O}(\operatorname{Alx}(P)) \rightarrow \mathcal{O}(\underline{L})(P)$ by $f^{-1}(U)=$ $l_{U}^{-1}(1)$. Taking $L=\mathcal{O}(\underline{L})(P)$ then yields the desired locale morphism.

5.1.25 Example Let $L$ be a locale object in the Kripke topos over a poset $P$. Internally, a point of $L$ is a locale morphism $1 \rightarrow L$, which is the same thing as an internal frame morphism $\mathcal{O}(L) \rightarrow \Omega$. Externally, one looks at $\Omega$ as the frame $\operatorname{Sub}(1)$ in Set. Since $\operatorname{Sub}(1) \cong \mathcal{O}(\operatorname{Alx}(P))$ in $[P$, Set $]$, one finds $\operatorname{Loc}([P$, Set $]) \cong$ Loc/Alx $(P)$. By Example 5.1.24, $L$ has an external description as a locale morphism $f: K \rightarrow L$, so that points in $L$ are described externally by sections of $f$, i.e. locale morphisms $g: L \rightarrow K$ satisfying $f \circ g=$ id. Compare the fact that $\mathcal{O}(\operatorname{Loc}(\mathbf{T}))=\operatorname{Sub}_{\mathbf{T}}(1)$ for any topos $\mathbf{T}$ to footnote 1 on page 40.

5.1.26 Locales already possess a logical aspect as well as a spatial one, as the logical perspective on complete Heyting algebras translates to the spatial perspective on locales. Elements $1 \rightarrow \mathcal{O}(L)$ of the Heyting algebra $\mathcal{O}(L)$ are the opens of the associated locale $L$, to be thought of as propositions, whereas points of the locale correspond to models of the logical theory defined by these propositions [212].

More precisely, recall that a formula is positive when it is built from atomic propositions by the connectives $\wedge$ and $\vee$ only, where $\vee$ but not $\wedge$ is allowed to be indexed by an infinite set. This can be motivated observationally: to verify a proposition $\bigvee_{i \in I} p_{i}$, one only needs to find a single $p_{i}$, whereas to verify $\bigwedge_{i \in I} p_{i}$ the validity of each $p_{i}$ needs to be established [5], an impossible task in practice when $I$ is infinite. A geometric formula then is one of the form $\varphi \Rightarrow \psi$, where $\varphi$ and $\psi$ are positive formulae.

Thus a frame $\mathcal{O}(L)$ defines a geometric propositional theory whose propositions correspond to opens in $L$, combined by logical connectives given by the lattice structure of $\mathcal{O}(L)$. Conversely, a propositional geometric theory $\mathfrak{T}$ has an associated Lindenbaum algebra $\mathcal{O}([\mathfrak{T}])$, defined as the poset of formulae of $\mathfrak{T}$ modulo provable equivalence, ordered by entailment. This poset turns out to be a frame, and the set-theoretical models of $\mathfrak{T}$ bijectively correspond to frame morphisms $\mathcal{O}([\mathfrak{T}]) \rightarrow\{0,1\}$. Identifying $\{0,1\}$ in Set with $\Omega=\mathcal{O}(1)$, one finds that a model of the theory $\mathfrak{T}$ is a point $1 \rightarrow[\mathfrak{T}]$ of the locale $[\mathfrak{T}]$. More generally, by Example 5.1 .24 one may consider a model of $\mathfrak{T}$ in a frame $\mathcal{O}(L)$ to be a locale morphism $L \rightarrow[\mathfrak{T}]$. 
5.1.27 Example Consider models of a geometric theory $\mathfrak{T}$ in a topos $\mathbf{T}$. Externally, these are given by locale morphisms $\operatorname{Loc}(\mathbf{T}) \rightarrow[\mathfrak{T}]$ [164, Theorem X.6.1 and Section IX.5]. One may also interpret $\mathfrak{T}$ in $\mathbf{T}$ and thus define a locale $[\mathfrak{T}]_{\mathbf{T}}$ internal to $\mathbf{T}$. The points of this locale, i.e. the locale morphisms $1 \rightarrow[\mathfrak{T}]_{\mathbf{T}}$ or frame morphisms $\mathcal{O}\left([\mathfrak{T}]_{\mathbf{T}}\right) \rightarrow \Omega$, describe the models of $\mathfrak{T}$ in $\mathbf{T}$ internally.

5.1.28 Example Several important internal number systems in Kripke toposes are defined by geometric propositional theories $\mathfrak{T}$, and can be computed via Example 5.1.17 and 5.1.18, Externally, the frame $\mathcal{O}([\mathfrak{T}])$ corresponding to the interpretation of $\mathfrak{T}$ in $[P$, Set $]$ is given by the functor $\mathcal{O}([\mathfrak{T}]): p \mapsto \mathcal{O}(\uparrow p \times[\mathfrak{T}])$ [45, Appendix A].

5.1.29 Example As an application of the previous example, we recall an explicit construction of the Dedekind real numbers (see [85] or [131, D4.7.4]. Define the propositional geometric theory $\mathfrak{T}_{\mathbb{R}}$ generated by formal symbols $(q, r) \in \mathbb{Q} \times \mathbb{Q}$ with $q<r$, ordered as $(q, r) \leq\left(q^{\prime}, r^{\prime}\right)$ iff $q^{\prime} \leq q$ and $r \leq r^{\prime}$, subject to the following relations:

$$
\begin{aligned}
& \left(q_{1}, r_{1}\right) \wedge\left(q_{2}, r_{2}\right)= \begin{cases}\left(\max \left(q_{1}, q_{2}\right), \min \left(r_{1}, r_{2}\right)\right) & \text { if } \max \left(q_{1}, q_{2}\right)<\min \left(r_{1}, r_{2}\right) \\
0 & \text { otherwise }\end{cases} \\
& (q, r)=\bigvee\left\{\left(q^{\prime}, r^{\prime}\right) \mid q<q^{\prime}<r^{\prime}<r\right\} \\
& 1=\bigvee\{(q, r) \mid q<r\} \\
& (q, r)=\left(q, r_{1}\right) \vee\left(q_{1}, r\right) \quad \text { if } q \leq q_{1} \leq r_{1} \leq r .
\end{aligned}
$$

This theory may be interpreted in any topos $\mathbf{T}$ with a natural numbers object, defining an internal locale $\mathbb{R}_{\mathbf{T}}$. Points $p$ of $\mathbb{R}_{\mathbf{T}}$, i.e. frame morphisms $p^{-1}: \mathcal{O}\left(\mathbb{R}_{\mathbf{T}}\right) \rightarrow \Omega$, correspond to Dedekind cuts $(L, U)$ by [164, p. 321]:

$$
\begin{aligned}
L & =\{q \in \mathbb{Q} \mid p \models(q, \infty)\} ; \\
U & =\{r \in \mathbb{Q} \mid p \models(-\infty, r)\},
\end{aligned}
$$

where $(q, \infty)$ and $(-\infty, r)$ are defined in terms of the formal generators of the frame $\mathcal{O}(\mathbb{Q})$ by $(q, \infty)=\bigvee\{(q, r) \mid q<r\}$ and $(-\infty, r)=\bigvee\{(q, r) \mid q<r\}$. The notation $p \models(q, r)$ means that $m^{-1}(q, r)$ is the subobject classifier $T: 1 \rightarrow \Omega$, where $(q, r)$ is seen as a morphism $1 \rightarrow \mathbb{Q} \times \mathbb{Q} \rightarrow \mathcal{O}\left(\mathbb{R}_{\mathbf{T}}\right)$. Conversely, a Dedekind cut $(L, U)$ uniquely determines a point $p$ by $(q, r) \mapsto \top$ iff $(q, r) \cap U \neq \emptyset$ and $(q, r) \cap L \neq \emptyset$. The Dedekind real numbers are therefore defined in any topos $\mathbf{T}$ as the subobject of $\mathcal{P}\left(\mathbb{Q}_{\mathbf{T}}\right) \times \mathcal{P}\left(\mathbb{Q}_{\mathbf{T}}\right)$ consisting of those $(L, U)$ that are points of $\mathbb{R}_{\mathbf{T}}$.

One may identify $\operatorname{Pt}\left(\mathbb{R}_{\text {Set }}\right)$ with the field $\mathbb{R}$ in the usual sense, and $\mathcal{O}\left(\mathbb{R}_{\text {Set }}\right)$ with the usual Euclidean topology on $\mathbb{R}$. 
In case $\mathbf{T}=[P$, Set $]$ for a poset $P$, one finds that $\mathcal{O}\left(\mathbb{R}_{\mathbf{T}}\right)$ is the functor $p \mapsto \mathcal{O}\left(\uparrow p \times \mathbb{R}_{\text {Set }}\right) ; c f$. Example 5.1.28. The latter set may be identified with the set of monotone functions $\uparrow p \rightarrow \mathcal{O}\left(\mathbb{R}_{\mathbf{S e t}}\right)$. When $P$ has a least element, the functor $\operatorname{Pt}\left(\mathbb{R}_{\mathbf{T}}\right)$ may be identified with the constant functor $p \mapsto \mathbb{R}_{\mathbf{S e t}}$.

\section{$5.2 C^{*}$-algebras}

This section considers a generalisation of the concept of topological space different from locales and toposes, namely so-called $C^{*}$-algebras [71, 135, 206]. These operator algebras also play a large role in quantum theory [101, 153, [196]. We first give a constructive definition of $C^{*}$-algebras that can be interpreted in any topos (with a natural numbers object), after [16-18].

5.2.1 In any topos (with a natural numbers object) the rationals $\mathbb{Q}$ can be interpreted [164, Section VI.8], as can the Gaussian rationals $\mathbb{C}_{\mathbb{Q}}=\{q+r i \mid q, r \in \mathbb{Q}\}$. For example, the interpretation of $\mathbb{C}_{\mathbb{Q}}$ in the Kripke topos over a poset $P$ is the constant functor that assigns the set $\mathbb{C}_{\mathbb{Q}}$ to each $p \in P$.

5.2.2 A monoid in Vect $_{K}$ for some $K \in$ Fld is called a (unital) $K$-algebra-not to be confused with Eilenberg-Moore algebras of a monad. It is called commutative when the multiplication of its monoid structure is. A *algebra is an algebra $A$ over an involutive field, together with an antilinear involution (_) $)^{*}: A \rightarrow A$.

More generally, we can think of an algebra as a monoid in a monoidal category with biproducts, and of a *algebra as a monoid in a dagger monoidal category with dagger biproducts [210].

5.2.3 Definition A seminorm on a *-algebra $A$ over $\mathbb{C}_{\mathbb{Q}}$ is a relation $N \subseteq A \times \mathbb{Q}^{+}$ satisfying

$$
\begin{aligned}
& (0, p) \in N, \\
& \exists_{q \in \mathbb{Q}^{+}} .(a, q) \in N, \\
(a, q) \in N \Rightarrow & \left(a^{*}, q\right) \in N, \\
(a, r) \in N \Longleftrightarrow & \exists_{q<r .} .(a, q) \in N, \\
(a, q) \in N \wedge(b, r) \in N \Rightarrow & (a+q, p+r) \in N, \\
(a, q) \in N \wedge(b, r) \in N \Rightarrow & (a b, q r) \in N, \\
(a, q) \in N \Rightarrow & (z a, q r) \in N \\
& (1, q) \in N
\end{aligned}
$$

for all $a, b \in A, q, r \in \mathbb{Q}^{+}$, and $z \in \mathbb{C}_{\mathbb{Q}}$. If this relation furthermore satisfies

$$
\left(a^{*} a, q^{2}\right) \in N \Longleftrightarrow(a, q) \in N
$$


for all $a \in A$ and $q \in \mathbb{Q}^{+}$, then $A$ is said to be a pre-semi-C*-algebra.

A seminorm $N$ is called a norm if $a=0$ whenever $(a, q) \in N$ for all $q \in \mathbb{Q}^{+}$. One can then formulate a suitable notion of completeness in this norm that does not rely on the axiom of choice, namely by considering Cauchy sequences of sets instead of Cauchy sequences [18]. A $C^{*}$-algebra is a pre-semi-C*-algebra $A$ whose seminorm is a norm in which $A$ is complete. Notice that a $C^{*}$-algebra by definition has a unit; what we defined as a $\mathrm{C}^{*}$-algebra is sometimes called a unital $\mathrm{C}^{*}$-algebra in the literature.

A morphism between $C^{*}$-algebras $A$ and $B$ is a linear function $f: A \rightarrow B$ satisfying $f(a b)=f(a) f(b), f\left(a^{*}\right)=f(a)^{*}$ and $f(1)=1$. C*-algebras and their morphisms form a category CStar. We denote its full subcategory of commutative $\mathrm{C}^{*}$-algebras by cCStar.

5.2.4 Classically, a seminorm induces a norm, and vice versa, by $(a, q) \in N$ if and only if $\|a\|<q$. Hence a $C^{*}$-algebra is precisely (the homset of) a one-object $\mathrm{C}^{*}$-category. The latter notion was introduced in Example 3.1.7.

5.2.5 The geometric theory $\mathfrak{T}_{\mathbb{R}}$ of Example 5.1 .29 can be extended to a geometric theory $\mathfrak{T}_{\mathbb{C}}$ describing the complexified locale $\mathbb{C}=\mathbb{R}+i \mathbb{R}$. There are also direct descriptions that avoid a defining role of $\mathbb{R}[18]$. In Set, the frame $\mathcal{O}(\mathbb{C})$ defined by $\mathfrak{T}_{\mathbb{C}}$ is the usual topology on the usual complex field $\mathbb{C}$. As a consequence of its completeness, a $C^{*}$-algebra is automatically an algebra over $\mathbb{C}$ (and not just over $\mathbb{C}_{\mathbb{Q}}$, as is inherent in the definition).

5.2.6 Example The continuous linear operators $\operatorname{Hilb}(H, H)$ on a Hilbert space $H$ form a C*-algebra. In fact, by the classical Gelfand-Naimark theorem, any $\mathrm{C}^{*}$ algebra can be embedded into one of this form [92]. Compare Theorem 3.7.18.

5.2.7 Example A locale $X$ is compact if every subset $S \subseteq X$ with $\bigvee S=1$ has a finite subset $F \subseteq S$ with $\bigvee F=1$. It is regular if $y=\bigvee(\downarrow y)$ for all $y \in X$, where $\Downarrow y=\{x \in X \mid x \ll y\}$ and $x \ll y$ iff there is a $z \in X$ with $z \wedge x=0$ and $z \vee y=1$. If the axiom of dependent choice is available—as in Kripke toposes [86] -then regular locales are automatically completely regular. Assuming the full axiom of choice, the category KRegLoc of compact regular locales in Set is equivalent to the category KHausTop of compact Hausdorff topological spaces. In general, if $X$ is a completely regular compact locale, then $C(X, \mathbb{C})$ is a commutative $\mathrm{C}^{*}$ algebra. In fact, the following theorem shows that all commutative $\mathrm{C}^{*}$-algebras are of this form. This so-called Gelfand duality justifies regarding C*-algebras as "noncommutative" generalisations of topological spaces [56].

5.2.8 Let us first mention two ways to characterise commutative $\mathrm{C}^{*}$-algebras classically. First, the category CStar is monoidal, so it makes sense to speak 
of monoids in it. It turns out that Mon $($ CStar $)=$ cCStar [120]. Compare Lemma 2.2.20. Second, a C*-algebra $A$ is commutative if and only if $a^{2}=0$ implies $a=0$ for all $a \in A$ [73, p. 288]. Compare Lemma 3.6.3.

5.2.9 Theorem [16-18] There is an equivalence

$$
\operatorname{cCStar} \underset{<\frac{\perp}{C\left({ }_{-}, \mathbb{C}\right)}}{\stackrel{\Sigma}{<}} \text { KRegLoc }{ }^{\mathrm{op}} \text {. }
$$

The locale $\Sigma(A)$ is called the Gelfand spectrum of $A$.

The previous theorem is proved in such a way that it applies in any topos. This means that we can give an explicit description of the Gelfand spectrum. The rest of this section is devoted to just that, following the reformulation by Thierry Coquand and Bas Spitters which is fully constructive [57, 58].

5.2.10 To motivate the following description, we mention that the classical proof [91, 92] defines $\Sigma(A)$ to be the set of characters of $A$, i.e. nonzero multiplicative functionals $\rho: A \rightarrow \mathbb{C}$. This set becomes a compact Hausdorff topological space by the sub-base consisting of $\left\{\rho \in \Sigma(A)|| \rho(a)-\rho_{0}(a) \mid<\varepsilon\right\}$ for $a \in A, \rho_{0} \in \Sigma$ and $\varepsilon>0$. A much simpler choice of sub-base would be $\mathcal{D}_{a}=\{\rho \in \Sigma \mid \rho(a)>0\}$ for $a \in A_{\mathrm{sa}}=\left\{a \in A \mid a^{*}=a\right\}$. Both the property that the $\rho$ are multiplicative and the fact that the $\mathcal{D}_{a}$ form a sub-base may then be expressed lattice-theoretically by letting $\mathcal{O}(\Sigma(A))$ be the frame freely generated by the formal symbols $\mathcal{D}_{a}$ for $a \in A_{\mathrm{sa}}$, subject to the relations

$$
\begin{aligned}
\mathcal{D}_{1} & =1 \\
\mathcal{D}_{a} \wedge \mathcal{D}_{-a} & =0 \\
\mathcal{D}_{-b^{2}} & =0 \\
\mathcal{D}_{a+b} & \leq \mathcal{D}_{a} \vee \mathcal{D}_{b}, \\
\mathcal{D}_{a b} & =\left(\mathcal{D}_{a} \wedge \mathcal{D}_{b}\right) \vee\left(\mathcal{D}_{-a} \wedge \mathcal{D}_{-b}\right),
\end{aligned}
$$

supplemented with the 'regularity rule'

$$
\mathcal{D}_{a} \leq \bigvee_{r \in \mathbb{Q}^{+}} \mathcal{D}_{a-r}
$$

5.2.11 Classically, the Gelfand transform $A \stackrel{\cong}{\rightrightarrows} C(\Sigma(A), \mathbb{C})$ is given by $a \mapsto \hat{a}$ with $\hat{a}(\rho)=\rho(a)$, and restricting to $A_{\mathrm{sa}}$ yields an isomorphism $A_{\mathrm{sa}} \cong C(\Sigma(A), \mathbb{R})$. Hence classically $\mathcal{D}_{a}=\{\rho \in \Sigma(A) \mid \hat{a}(\rho)>0\}$, which equals $\operatorname{supp}(\hat{a})$ as defined in Example 2.2.17 (the literature sometimes defines the support of a function as 
the closure of what we call its support). In a constructive setting, we must associate a locale morphism $\hat{a}: \Sigma(A) \rightarrow \mathbb{R}$ to each $a \in A_{\mathrm{sa}}$, which is, by definition, a frame morphism $\hat{a}^{-1}: \mathcal{O}(\mathbb{R}) \rightarrow \mathcal{O}(\Sigma(A))$. Aided by the intuition of 5.2.10, one finds that $\hat{a}^{-1}(-\infty, s)=\mathcal{D}_{s-a}$ and $\hat{a}^{-1}(r, \infty)=\mathcal{D}_{a-r}$ for basic opens. Hence $\hat{a}^{-1}(r, s)=\mathcal{D}_{s-a} \wedge \mathcal{D}_{a-r}$ for rationals $r<s$. By Example 5.1.29, we have $A_{\mathrm{sa}} \cong C(\Sigma(A), \mathbb{R})=\Gamma\left(\operatorname{Pt}(\mathbb{R})_{\operatorname{Sh}(\Sigma(A))}\right)$, where $\Gamma$ is the global sections functor. Hence, $A_{\mathrm{sa}}$ is isomorphic (through the Gelfand transform) to the global sections of the real numbers in the topos of sheaves on its spectrum (and $A$ itself "is" the complex numbers in the same sense).

5.2.12 To describe the Gelfand spectrum more explicitly, we start with the distributive lattice $L_{A}$ freely generated by the formal symbols $\mathrm{D}_{a}$ for $a \in A_{\mathrm{sa}}$, subject to the relations (5.3)-(5.7). Being an involutive rig, $A_{\mathrm{sa}}$ has a positive cone $A^{+}=\left\{a \in A_{\mathrm{sa}} \mid a \geq 0\right\}=\left\{a^{2} \mid a \in A_{\mathrm{sa}}\right\}$ by 3.5.1. (For $A=\mathbf{H i l b}(H, H)$, one has $a \in A^{+}$iff $\langle x \mid a(x)\rangle \geq 0$ for all $x \in H$.) The given definition of $A^{+}$induces a partial order $\leq$ on $A^{+}$by $a \leq b$ iff $0 \leq a-b$, with respect to which $A^{+}$is a distributive lattice. Now we define a partial order $\preccurlyeq$ on $A^{+}$by $a \preccurlyeq b$ iff $a \leq n b$ for some $n \in \mathbb{N}$. Define an equivalence relation on $A^{+}$by $a \approx b$ iff $a \preccurlyeq b$ and $b \preccurlyeq a$. The lattice operations on $A^{+}$respect $\approx$ and hence $A^{+} / \approx$ is a lattice. We have

$$
L_{A} \cong A^{+} / \approx .
$$

The image of the generator $\mathrm{D}_{a}$ in $L_{A}$ corresponds to the equivalence class $\left[a^{+}\right]$ in $A^{+} / \approx$, where $a=a^{+}-a^{-}$with $a^{ \pm} \in A^{+}$in the usual way. Theorem 5.3.12 will show that the lattice $L_{A}$ can be computed locally in certain Kripke toposes. In preparation, we now work towards Lemma 5.2.17below.

5.2.13 Extending the geometric propositional logic of 5.1.26, the positive formulae of a geometric predicate logic may furthermore involve finitely many free variables and the existential quantifier $\exists$, and its axioms take the form $\forall_{x \in X} . \varphi(x) \Rightarrow \psi(x)$ for positive formulae $\varphi, \psi$. Geometric formulae form an important class of logical formulae, because they are precisely the ones whose truth value is preserved by inverse images of geometric morphisms between toposes. From their syntactic form alone, it follows that their external interpretation is determined locally in Kripke toposes, as the following lemma shows.

5.2.14 Lemma [131] Corollary D1.2.14] Let $\mathfrak{T}$ be a geometric theory, and denote the category of its models in a topos $\mathbf{T}$ by $\operatorname{Model}(\mathfrak{T}, \mathbf{T})$. For any category $\mathbf{C}$, there is a canonical isomorphism of categories $\operatorname{Model}(\mathfrak{T},[\mathbf{C}, \mathbf{S e t}]) \cong$ $[\mathrm{C}, \operatorname{Model}(\mathfrak{T}, \operatorname{Set})]$. 
5.2.15 Definition A Riesz space is a vector space $R$ over $\mathbb{R}$ that is simultaneously a distributive lattice, such that $f \leq g$ implies $f+h \leq g+h$ for all $h$, and $f \geq 0$ implies $r f \geq 0$ for all $r \in \mathbb{R}^{+}$[160, Definition 11.1].

An $f$-algebra is a commutative $\mathbb{R}$-algebra $R$ whose underlying vector space is a Riesz space in which $f, g \geq 0$ implies $f g \geq 0$, and $f \wedge g=0$ implies $h f \wedge g=0$ for all $h \geq 0$. Moreover, the multiplicative unit 1 has to be strong in the sense that for each $f \in R$ one has $-n 1 \leq f \leq n 1$ for some $n \in \mathbb{N}$ [217, Definition 140.8].

5.2.16 Example If $A$ is a commutative $\mathrm{C}^{*}$-algebra, then $A_{\mathrm{sa}}$ becomes an $\mathrm{f}$ algebra over $\mathbb{R}$ under the order defined in 5.2.12. Conversely, by the StoneYosida representation theorem every f-algebra over $\mathbb{R}$ can be densely embedded in $C(X, \mathbb{R})$ for some compact locale $X$ [60].

Clearly, the real field $\mathbb{R}$ with its usual order is an f-algebra. The spectrum of an $\mathrm{f}$-algebra $R$ is classically defined as the space of all its representations [217]; a representation of $R$ is a linear function $\sigma: R \rightarrow \mathbb{R}$ satisfying $\sigma(1)=1$ and $\sigma(f \vee g)=\sigma(f) \vee \sigma(g)$. This can also be defined constructively as a (compact, completely regular) locale, whose points are precisely the representations, in a way very similar to 5.2.10 [60].

For a commutative $\mathrm{C}^{*}$-algebra $A$, its Gelfand spectrum coincides with the spectrum of $A_{\mathrm{sa}}$ as an f-algebra, as in part (a) of the following lemma.

5.2.17 Lemma Let $A$ be a commutative $C^{*}$-algebra.

(a) The Gelfand spectrum of $A$ coincides with the spectrum of the f-algebra $A_{\mathrm{sa}}$.

(b) The theory of f-algebras is geometric.

Proof Part (a) is proven in [58]. For (b), notice that an f-algebra over $\mathbb{Q}$ is precisely a uniquely divisible lattice-ordered ring [57, p. 151], since unique divisibility turns a ring into a $\mathbb{Q}$-algebra ( $c f$. Example 2.5.2). The definition of a lattice-ordered ring can be written using equations only. The theory of torsionfree rings, i.e. if $n>0$ and $n x=0$ then $x=0$, is also algebraic. The theory of divisible rings is obtained by adding infinitely many geometric axioms $\exists_{y} . n y=x$, one for each $n>0$, to the algebraic theory of rings. Finally, a torsion-free divisible ring is the same as a uniquely divisible ring: if $n y=x$ and $n z=x$, then $n(y-z)=0$, so that $y-z=0$. We conclude that the theory of uniquely divisible lattice-ordered rings, i.e. f-algebras, is geometric, establishing (b).

5.2.18 Proposition The lattice $L_{A}$ generating the spectrum of a commutative $C^{*}$ algebra $A$ is preserved under inverse images of geometric morphisms.

Proof By the previous lemma, $A_{\mathrm{sa}}$ and hence $A^{+}$are definable by a geometric theory. Since the relation $\approx$ of 5.2 .12 is defined by an existential quantification, $L_{A} \cong A^{+} / \approx$ is preserved under inverse images of geometric morphisms. 
We now turn to the regularity condition (5.8), which is to be imposed on $L_{A}$. This condition turns out to be a special case of the relation $\ll$ (see Example 5.2.7).

5.2.19 Lemma For all $\mathrm{D}_{a}, \mathrm{D}_{b} \in L_{A}$ the following are equivalent:

(a) There exists $\mathrm{D}_{c}$ with $\mathrm{D}_{c} \vee \mathrm{D}_{a}=1$ and $\mathrm{D}_{c} \wedge \mathrm{D}_{b}=0$;

(b) There exists a rational $q>0$ with $\mathrm{D}_{b} \leq \mathrm{D}_{a-q}$.

Proof Assuming (a), there exists a rational $q>0$ with $\mathrm{D}_{c-q} \vee \mathrm{D}_{a-q}=1$ by [57, Corollary 1.7]. Hence $\mathrm{D}_{c} \vee \mathrm{D}_{a-q}=1$, so $\mathrm{D}_{b}=\mathrm{D}_{b} \wedge\left(\mathrm{D}_{c} \vee \mathrm{D}_{a-q}\right)=\mathrm{D}_{b} \wedge \mathrm{D}_{a-q} \leq \mathrm{D}_{a-q}$, establishing (b). For the converse, choose $\mathrm{D}_{c}=D_{q-a}$.

5.2.20 In view of the above lemma, we henceforth write $\mathrm{D}_{b} \ll \mathrm{D}_{a}$ if there exists a rational $q>0$ such that $\mathrm{D}_{b} \leq \mathrm{D}_{a-q}$, and note that the regularity condition (5.8) just states that the frame $\mathcal{O}(\Sigma(A))$ is regular [57].

We recall that an ideal of a lattice $L$ is a lower set $U \subseteq L$ that is closed under finite joins; the collection of all ideals in $L$ is denoted by $\operatorname{Idl}(L)$. An ideal $U$ of a distributive lattice $L$ is regular when $\downarrow x \subseteq U$ implies $x \in U$. Any ideal $U$ can be turned into a regular ideal $\bar{U}$ by means of the closure operator $\overline{\left(\_\right)}: D L \rightarrow D L$ defined by $\bar{U}=\{x \in L \mid \forall y \in L . y \ll x \Rightarrow y \in U\}$ [46], with a canonical inclusion as in Proposition 5.1.11.

5.2.21 Theorem The Gelfand spectrum $\mathcal{O}(\Sigma(A))$ of a commutative $C^{*}$-algebra $A$ is isomorphic to the frame of all regular ideals of $L_{A}$, i.e.

$$
\mathcal{O}(\Sigma(A)) \cong\left\{U \in \operatorname{Idl}\left(L_{A}\right) \mid\left(\forall_{\mathrm{D}_{b} \in L_{A}} \cdot \mathrm{D}_{b} \ll \mathrm{D}_{a} \Rightarrow \mathrm{D}_{b} \in U\right) \Rightarrow \mathrm{D}_{a} \in U\right\} .
$$

In this realisation, the canonical map $f: L_{A} \rightarrow \mathcal{O}(\Sigma(A))$ is given by

$$
f\left(\mathrm{D}_{a}\right)=\left\{\mathrm{D}_{c} \in L_{A} \mid \forall \mathrm{D}_{b} \in L_{A} . \mathrm{D}_{b} \ll \mathrm{D}_{c} \Rightarrow \mathrm{D}_{b} \leq \mathrm{D}_{a}\right\} .
$$

Proof For a commutative $C^{*}$-algebra $A$, the lattice $L_{A}$ is strongly normal [57, Theorem 1.11], and hence normal. (A distributive lattice is normal if for all $b_{1}, b_{2}$ with $b_{1} \vee b_{2}=1$ there are $c_{1}, c_{2}$ such that $c_{1} \wedge c_{2}=0$ and $c_{1} \vee b_{1}=1$ and $c_{2} \vee b_{2}=$ 1.) By [46, Theorem 27], regular ideals in a normal distributive lattice form a compact regular frame. The result now follows from [57, Theorem 1.11].

5.2.22 Corollary The Gelfand spectrum of a commutative $C^{*}$-algebra $A$ is given by

$$
\mathcal{O}(\Sigma(A)) \cong\left\{U \in \operatorname{Idl}\left(L_{A}\right) \mid \forall_{a \in A_{\mathrm{sa}}} \forall_{q>0} \cdot \mathrm{D}_{a-q} \in U \Rightarrow \mathrm{D}_{a} \in U\right\} .
$$

Proof By combining Lemma 5.2.19 with Theorem 5.2.21. 
The following theorem is the key to explicitly determining the external description of the Gelfand spectrum $\mathcal{O}(\Sigma(A))$ of a $\mathrm{C}^{*}$-algebra $A$ in a topos.

5.2.23 Theorem For a commutative $C^{*}$-algebra A, define a covering relation $\triangleleft$ on $L_{A}$ by $x \triangleleft U$ iff $f(x) \leq \bigvee f(U)$, in the notation of Theorem 5.2.21.

(a) One has $\mathcal{O}(\Sigma(A)) \cong \mathcal{F}\left(L_{A}, \triangleleft\right)$, under which $\mathcal{D}_{a} \mapsto \downarrow D_{a}$.

(b) Then $\mathrm{D}_{a} \triangleleft U$ iff for all rational $q>0$ there is a (Kuratowski) finite $U_{0} \subseteq U$ such that $\mathrm{D}_{a-q} \leq \bigvee U_{0}$.

PRoof Part (a) follows from Proposition 5.1.11. For (b), first assume $\mathrm{D}_{a} \triangleleft U$, and let $q \in \mathbb{Q}$ satisfy $q>0$. From (the proof of) Lemma 5.2.17 we have $\mathrm{D}_{a} \vee$ $\mathrm{D}_{q-a}=1$, whence $\bigvee f(U) \vee f\left(\mathrm{D}_{q-a}\right)=1$. Because $\mathcal{O}(\Sigma(A))$ is compact, there is a finite $U_{0} \subseteq U$ for which $\bigvee f\left(U_{0}\right) \vee f\left(\mathrm{D}_{q-a}\right)=1$. Since $f\left(\mathrm{D}_{a}\right)=1$ if and only if $\mathrm{D}_{a}=1$ by Theorem 5.2.21, we have $\mathrm{D}_{b} \vee \mathrm{D}_{q-a}=1$, where $\mathrm{D}_{b}=\bigvee U_{0}$. By (5.4), we have $\mathrm{D}_{a-q} \wedge \mathrm{D}_{q-a}=0$, and hence

$$
\mathrm{D}_{a-q}=\mathrm{D}_{a-q} \wedge 1=\mathrm{D}_{a-q} \wedge\left(\mathrm{D}_{b} \vee \mathrm{D}_{q-a}\right)=\mathrm{D}_{a-q} \wedge \mathrm{D}_{b} \leq \mathrm{D}_{b}=\bigvee U_{0} .
$$

For the converse, notice that $f\left(\mathrm{D}_{a}\right) \leq \bigvee\left\{f\left(\mathrm{D}_{a-q}\right) \mid q \in \mathbb{Q}, q>0\right\}$ by construction. So from the assumption we have $f\left(\mathrm{D}_{a}\right) \leq \bigvee f(U)$ and hence $\mathrm{D}_{a} \triangleleft U$.

\subsection{Bohrification}

This section explains the technique of Bohrification. For a (generally) noncommutative $\mathrm{C}^{*}$-algebra $A$, Bohrification constructs a topos in which $A$ becomes commutative. More precisely, to any $\mathrm{C}^{*}$-algebra $A$, we associate a particular commutative $\mathrm{C}^{*}$-algebra $\underline{A}$ in the Kripke topos $[\mathcal{C}(A)$, Set], where $\mathcal{C}(A)$ is the set of commutative $\mathrm{C}^{*}$-subalgebras of $A$. By Gelfand duality, the commutative $\mathrm{C}^{*}$-algebra $\underline{A}$ has a spectrum $\Sigma(\underline{A})$, which is a locale in $[\mathcal{C}(A)$, Set $]$.

5.3.1 To introduce the idea, we outline the general method of Bohrification. We will subsequently give concrete examples.

Let $\mathfrak{T}_{1}$ and $\mathfrak{T}_{2}$ be geometric theories whose variables range over only one type, apart from constructible types such as $\mathbb{N}$ and $\mathbb{Q}$. Suppose that $\mathfrak{T}_{1}$ is a subtheory of $\mathfrak{T}_{2}$. There is a functor $\mathcal{C}: \operatorname{Model}\left(\mathfrak{T}_{1}\right.$, Set $) \rightarrow$ Poset, defined on objects as $\mathcal{C}(A)=\left\{C \subseteq A \mid C \in \operatorname{Model}\left(\mathfrak{T}_{2}\right.\right.$, Set $\left.)\right\}$, ordered by inclusion. On a morphism $f: A \rightarrow B$ of $\operatorname{Model}\left(\mathfrak{T}_{1}\right.$, Set $)$, the functor $\mathcal{C}$ acts as $\mathcal{C}(f): \mathcal{C}(A) \rightarrow \mathcal{C}(B)$ by the direct image $C \mapsto f(C)$. Hence, there is a functor $\mathcal{T}: \operatorname{Model}\left(\mathfrak{T}_{1}\right.$, Set $) \rightarrow$ Topos, defined on objects by $\mathcal{T}(A)=[\mathcal{C}(A)$, Set $]$ and determined on morphisms by $\mathcal{T}(f)^{*}=\left({ }_{-}\right) \circ \mathcal{C}(f)$. Define the canonical 
object $\underline{A} \in \mathcal{T}(A)$ by $\underline{A}(C)=C$, acting on a morphism $D \subseteq C$ of $\mathcal{C}(A)$ as the inclusion $\underline{A}(D) \hookrightarrow \underline{A}(C)$. Then $\underline{A}$ is a model of $\mathfrak{T}_{2}$ in the Kripke topos $\mathcal{T}(A)$ by Lemma 5.2.14,

5.3.2 Example Let $\mathfrak{T}_{1}$ be the theory of groups, and $\mathfrak{T}_{2}$ the theory of Abelian groups. Both are geometric theories, and $\mathfrak{T}_{1}$ is a subtheory of $\mathfrak{T}_{2}$. Then $\mathcal{C}(G)$ is the collection of Abelian subgroups $C$ of $G$, ordered by inclusion, and the functor $\underline{G}: C \mapsto C$ is an Abelian group in $\mathcal{T}(G)=[\mathcal{C}(G)$, Set $]$.

We now turn to the setting of our interest: (commutative) $\mathrm{C}^{*}$-algebras. As the theory of $\mathrm{C}^{*}$-algebras is not geometric, it does not follow from the arguments of 5.3.1 that $\underline{A}$ will be a commutative $\mathrm{C}^{*}$-algebra in $\mathcal{T}(A)$. Theorem 5.3.8 below will show that the latter is nevertheless true.

5.3.3 Proposition There is a functor $\mathcal{C}:$ CStar $\rightarrow$ Poset, defined on objects as

$$
\mathcal{C}(A)=\left\{C \in \mathbf{c C S t a r} \mid C \text { is a } C^{*} \text {-subalgebra of } A\right\},
$$

ordered by inclusion. Its action $\mathcal{C}(f): \mathcal{C}(A) \rightarrow \mathcal{C}(B)$ on a morphism $f: A \rightarrow B$ of CStar is the direct image $C \mapsto f(C)$. Hence, there is a functor $\mathcal{T}:$ CStar $\rightarrow$ Topos, defined by $\mathcal{T}(A)=[\mathcal{C}(A)$, Set $]$ on objects and $\mathcal{T}(f)^{*}=\left({ }_{-}\right) \circ \mathcal{C}(f)$ on morphisms.

ProOF It suffices to show that $\mathcal{T}(f)^{*}$ is part of a geometric morphism, which follows from [164, Theorem VII.2.2].

5.3.4 Example The following example determines $\mathcal{C}(A)$ for $A=\operatorname{Hilb}\left(\mathbb{C}^{2}, \mathbb{C}^{2}\right)$, the $C^{*}$-algebra of complex 2 by 2 matrices. Any $C^{*}$-algebra has a single onedimensional commutative $C^{*}$-subalgebra, namely $\mathbb{C}$, the scalar multiples of the unit. Furthermore, any two-dimensional $C^{*}$-subalgebra is generated by a pair of orthogonal one-dimensional projections. The one-dimensional projections in $A$ are of the form

$$
p(x, y, z)=\frac{1}{2}\left(\begin{array}{cc}
1+x & y+i z \\
y-i z & 1-x
\end{array}\right),
$$

where $(x, y, z) \in \mathbb{R}^{3}$ satisfies $x^{2}+y^{2}+z^{2}=1$. Thus the one-dimensional projections in $A$ are precisely parametrised by $S^{2}$. Since $1-p(x, y, z)=p(-x,-y,-z)$, and pairs $(p, 1-p)$ and $(1-p, p)$ define the same $\mathrm{C}^{*}$-subalgebra, the twodimensional elements of $\mathcal{C}(A)$ are parametrised by $S^{2} / \sim$, where $(x, y, z) \sim$ $(-x,-y,-z)$. This space, in turn, is homeomorphic with the real projective plane $\mathbb{R P}^{2}$, i.e. the set of lines in $\mathbb{R}^{3}$ passing through the origin ${ }^{3}$ Parametrising

\footnotetext{
${ }^{3}$ This space has an interesting topology that is quite different from the Alexandrov topology on $\mathcal{C}(A)$, but that we nevertheless ignore.
} 
$\mathcal{C}(A) \cong\{\mathbb{C}\}+\mathbb{R P}^{2}$, a point $[x, y, z] \in S^{2} / \sim$ then corresponds to the $\mathrm{C}^{*}$-algebra $C_{[x, y, z]}$ generated by the projections $\{p(x, y, z), p(-x,-y,-z)\}$. The order of $\mathcal{C}(A)$ is flat: $C<D$ iff $C=\mathbb{C}$.

5.3.5 Example We now generalise the previous example to $A=\operatorname{Hilb}\left(\mathbb{C}^{n}, \mathbb{C}^{n}\right)$ for any $n \in \mathbb{N}$. In general, one has $\mathcal{C}(A)=\coprod_{k=1}^{n} \mathcal{C}(k, n)$, where $\mathcal{C}(k, n)$ denotes the collection of all $k$-dimensional commutative unital $\mathrm{C}^{*}$-subalgebras of $A$. To parametrise $\mathcal{C}(k, n)$, we first show that each of its elements $C$ is a unitary rotation $C=U D U^{*}$, where $U \in S U(n)$ and $D$ is some subalgebra contained in the algebra of all diagonal matrices. This follows from the case $k=n$, since each element of $\mathcal{C}(k, n)$ with $k<n$ is contained in some maximal commutative subalgebra. For $k=n$, note that $C \in \mathcal{C}(n, n)$ is generated by $n$ mutually orthogonal projections $p_{1}, \ldots, p_{n}$ of rank 1 . Each $p_{i}$ has a single unit eigenvector $u_{i}$ with eigenvalue 1 ; its other eigenvalues are 0 . Put these $u_{i}$ as columns in a matrix, called $U$. Then $U^{*} p_{i} U$ is diagonal for all $i$, for if $\left(e_{i}\right)$ is the standard basis of $\mathbb{C}^{n}$, then $U e_{i}=u_{i}$ for all $i$ and hence $U^{*} p_{i} U e_{i}=U^{*} p_{i} u_{i}=U^{*} u_{i}=e_{i}$, while for $i \neq j$ one finds $U^{*} p_{i} U e_{j}=0$. Hence the matrix $U^{*} p_{i} U$ has a one at location $i i$ and zero's everywhere else. All other elements $a \in C$ are functions of the $p_{i}$, so that $U^{*} a U$ is equally well diagonal. Hence $C=U D_{n} U^{*}$, with $D_{n}$ the algebra of all diagonal matrices. Thus

$$
\mathcal{C}(n, n)=\left\{U D_{n} U^{*} \mid U \in S U(n)\right\},
$$

with $D_{n}=\left\{\operatorname{diag}\left(a_{1}, \ldots, a_{n}\right) \mid a_{i} \in \mathbb{C}\right\}$, and $\mathcal{C}(k, n)$ for $k<n$ is obtained by partitioning $\{1, \ldots, n\}$ into $k$ nonempty parts and demanding $a_{i}=a_{j}$ for $i, j$ in the same part. However, because of the conjugation with arbitrary $U \in S U(n)$, two such partitions induce the same subalgebra precisely when they permute parts of equal size. Such permutations may be handled using Young tableaux [90]. As the size of a part is of more interest than the part itself, we define

$$
Y(k, n)=\left\{\left(i_{1}, \ldots, i_{k}\right) \mid 0<i_{1}<i_{2}<\cdots<i_{k}=n, i_{j+1}-i_{j} \leq i_{j}-i_{j-1}\right\}
$$

(where $i_{0}=0$ ) as the set of partitions inducing different subalgebras. Hence

$$
\begin{aligned}
\mathcal{C}(k, n) \cong\left\{\left(p_{1}, \ldots, p_{k}\right):\right. & p_{j} \in \operatorname{Proj}(A), \quad\left(i_{1}, \ldots, i_{k}\right) \in Y(k, n) \\
\mid & \left.\operatorname{dim}\left(\operatorname{Im}\left(p_{j}\right)\right)=i_{j}-i_{j-1}, \quad p_{j} \wedge p_{j^{\prime}}=0 \text { for } j \neq j^{\prime}\right\} .
\end{aligned}
$$

Now, since $d$-dimensional orthogonal projections in $\mathbb{C}^{n}$ bijectively correspond to 
the $d$-dimensional (closed) subspaces of $\mathbb{C}^{n}$ they project onto, we can write

$$
\begin{gathered}
\mathcal{C}(k, n) \cong\left\{\left(V_{1}, \ldots, V_{k}\right):\left(i_{1}, \ldots, i_{k}\right) \in Y(k, n), V_{j} \in \operatorname{Gr}\left(i_{j}-i_{j-1}, n\right)\right. \\
\left.\mid V_{j} \cap V_{j^{\prime}}=0 \text { for } j \neq j^{\prime}\right\},
\end{gathered}
$$

where $\operatorname{Gr}(d, n)=U(n) /(U(d) \times U(n-d))$ is the well-known Grassmannian, i.e. the set of all $d$-dimensional subspaces of $\mathbb{C}^{n}$ [97]. In terms of the partial flag manifold

$$
\mathrm{G}\left(i_{1}, \ldots, i_{k} ; n\right)=\prod_{j=1}^{k} \operatorname{Gr}\left(i_{j}-i_{j-1}, n-i_{j-1}\right),
$$

for $\left(i_{1}, \ldots, i_{k}\right) \in Y(k, n)$ (see [90]), we finally obtain

$$
\mathcal{C}(k, n) \cong\{V \in \mathrm{G}(i ; n): i \in Y(k, n)\} / \sim,
$$

where $i \sim i^{\prime}$ if one arises from the other by permutations of equal-sized parts.

This is indeed generalises the previous example $n=2$. First, for any $n$ the set $\mathcal{C}(1, n)$ has a single element, as there is only one Young tableau for $k=1$. Second, we have $Y(2,2)=\{(1,2)\}$, so that

$$
\mathcal{C}(2,2) \cong(\operatorname{Gr}(1,2) \times \operatorname{Gr}(1,1)) / S(2) \cong \operatorname{Gr}(1,2) / S(2) \cong \mathbb{C P}^{1} / S(2) \cong \mathbb{R P}^{2} .
$$

5.3.6 Definition Let $A$ be a $C^{*}$-algebra. Define the functor $\underline{A}: \mathcal{C}(A) \rightarrow$ Set by acting on objects as $\underline{A}(C)=C$, and acting on morphisms $C \subseteq D$ of $\mathcal{C}(A)$ as the inclusion $\underline{A}(C) \hookrightarrow \underline{A}(D)$. We call $\underline{A}$, or the process of obtaining it, the Bohrification of $A$.

5.3.7 Convention We will underline entities internal to $\mathcal{T}(A)$ to distinguish between the internal and external points of view.

The particular object $\underline{A}$ turns out to be a commutative $\mathrm{C}^{*}$-algebra in the topos $\mathcal{T}(A)$, even though the theory of $\mathrm{C}^{*}$-algebras is not geometric. This resembles the so-called "microcosm principle", according to which structure of an internal entity depends on similar structure of the ambient category [13, 109].

5.3.8 Theorem Operations inherited from A make $\underline{A}$ a commutative $C^{*}$-algebra in $\mathcal{T}(A)$. More precisely, $\underline{A}$ is a vector space over the complex field $\operatorname{Pt}(\underline{\mathbb{C}}): C \mapsto \mathbb{C}$ by

$$
\begin{array}{lll}
0: \underline{1} \rightarrow \underline{A}, & +: \underline{A} \times \underline{A} \rightarrow \underline{A}, & \cdot: \operatorname{Pt}(\underline{\mathbb{C}}) \times \underline{A} \rightarrow \underline{A}, \\
0_{C}(*)=0, & a+{ }_{C} b=a+b, & z \cdot{ }_{C} a=z \cdot a,
\end{array}
$$


and an involutive algebra through

$$
\begin{array}{ll}
\cdot: \underline{A} \times \underline{A} \rightarrow \underline{A}, & ()^{*}: \underline{A} \rightarrow \underline{A} \\
a \cdot C b=a \cdot b, & \left(a^{*}\right)_{C}=a^{*} .
\end{array}
$$

The norm relation is the subobject $N \in \operatorname{Sub}\left(\underline{A} \times \underline{\mathbb{Q}^{+}}\right)$given by

$$
N_{C}=\left\{(a, q) \in C \times \mathbb{Q}^{+} \mid\|a\|<q\right\} .
$$

Proof Recall (Definition 5.2.3) that a pre-semi-C*-algebra is a C*-algebra that is not necessarily Cauchy complete, and whose seminorm is not necessarily a norm. Since the theory of pre-semi-C*-algebras is geometric, Lemma 5.2.14 shows that $\underline{A}$ is a commutative pre-semi-C*-algebra in $\mathcal{T}(A)$, as in 5.3.1. Let us prove that $\underline{A}$ is in fact a pre-C*-algebra, i.e. that the seminorm is a norm. It suffices to show that $C \Vdash \forall_{a \in \underline{A}_{\mathrm{sa}}} \forall_{q \in Q^{+}} .(a, q) \in N \Rightarrow a=0$ for all $C \in \mathcal{C}(A)$. By 5.1 .20 , this means

for all $C^{\prime} \supseteq C$ and $a \in C^{\prime}$, if $C^{\prime} \Vdash \forall_{q \in \underline{\mathbb{Q}^{+}}} .(a, q) \in N$, then $C^{\prime} \Vdash a=0$, i.e. for all $C^{\prime} \supseteq C$ and $a \in C^{\prime}$, if $C^{\prime \prime} \Vdash(a, q) \in N$ for all $C^{\prime \prime} \supseteq C^{\prime}$ and $q \in \mathbb{Q}^{+}$, then $C^{\prime} \Vdash a=0$,

i.e. for all $C^{\prime} \supseteq C$ and $a \in C^{\prime}$, if $\|a\|=0$, then $a=0$.

But this holds, since every $C^{\prime}$ is a $\mathrm{C}^{*}$-algebra.

Finally, we prove that $\underline{A}$ is in fact a $\mathrm{C}^{*}$-algebra. Since the axiom of dependent choice holds in $\mathcal{T}(A)$ [86], it suffices to prove that every regular Cauchy sequence converges, where a sequence $\left(x_{n}\right)$ is regular Cauchy when $\left\|x_{n}-x_{m}\right\| \leq$ $2^{-n}+2^{-m}$ for all $n, m \in \mathbb{N}$. Thus we need to prove

$$
C \Vdash \forall_{n, m \in \mathbb{\mathbb { N }}} \cdot\left\|x_{n}-x_{m}\right\| \leq 2^{-n}+2^{-m} \Rightarrow \exists_{x \in \underline{A}} \cdot \forall_{n \in \mathbb{N}} \cdot\left\|x-x_{n}\right\| \leq 2^{-n},
$$

i.e. for all $C^{\prime} \supseteq C$, if $C^{\prime} \Vdash\left(\forall_{n, m \in \mathbb{N}} \cdot\left\|x_{n}-x_{m}\right\| \leq 2^{-n}+2^{-m}\right)$,

$$
\text { then } C^{\prime} \Vdash \exists_{x \in \underline{A}} \cdot \forall_{n \in \underline{\mathbb{N}}} \cdot\left\|x-x_{n}\right\| \leq 2^{-n} \text {, }
$$

i.e. for all $C^{\prime} \supseteq C$, if $C^{\prime} \Vdash$ “ $(x)_{n}$ is regular", then $C^{\prime} \Vdash$ “ $(x)_{n}$ converges”.

Once again, this holds because every $C^{\prime}$ is a $C^{*}$-algebra.

5.3.9 Applying 5.2 .9 to the commutative $\mathrm{C}^{*}$-algebra $\underline{A}$ in the topos $\mathcal{T}(A)$, we obtain a locale $\underline{\Sigma}(\underline{A})$ in that topos. As argued in Chapter $1, \underline{\Sigma}(\underline{A})$ is the 'state space' carrying the logic of the physical system whose observable algebra is $A$.

An important property of $\underline{\Sigma}(\underline{A})$ is that it is typically highly non-spatial, as the following theorem proves. This theorem is a localic extension of a topostheoretic reformulation of the Kochen-Specker theorem [143] due to Jeremy 
Butterfield and Chris Isham [39-42].

5.3.10 Theorem Let $H$ be a Hilbert space with $\operatorname{dim}(H)>2$, and $A=\operatorname{Hilb}(H, H)$. The locale $\underline{\Sigma}(\underline{A})$ has no points.

Proof The real number object in $\mathcal{T}(A)$ is a ring (in $\mathcal{T}(A)$ ), so that it makes sense to talk about multiplicative functionals (on the $\mathrm{C}^{*}$-algebra $\underline{A}$ in $\mathcal{T}(A)$ ). It turns out that the points of $\underline{\Sigma}(\underline{A})$, should they exist, are precisely the multiplicative functionals on $\underline{A}$ (see [18], who explicitly use only topos-valid constructions and reasoning). So for any point $\underline{\rho}: \underline{1} \rightarrow \underline{\Sigma}(\underline{A})$ of the locale $\underline{\Sigma}(\underline{A})$ there is a map $\underline{V}_{\rho}: \underline{A}_{\mathrm{sa}} \rightarrow \operatorname{Pt}(\underline{\mathbb{R}})$. Being a morphism in $\mathcal{T}(A)$, the map $\underline{V}_{\rho}$ is a natural transformation, with components $\underline{V}_{\rho}(C): \underline{A}_{\mathrm{sa}}(C) \rightarrow \mathrm{Pt}(\underline{\mathbb{R}})(C)$; by Definition 5.3.6 and Example 5.1.29, this is just $\underline{V}_{\rho}(C): C_{\mathrm{sa}} \rightarrow \mathbb{R}$. Hence one has a multiplicative functional $\underline{V}_{\rho}(C)$ for each $C \in \mathcal{C}(A)$ in the usual sense, with the naturality, or 'noncontextuality', property that if $C \subseteq D$, then the restriction of $\underline{V}_{\rho}(D)$ to $C_{\text {sa }}$ is $\underline{V}_{\rho}(C)$. But that is precisely the kind of function on $\operatorname{Hilb}(H, H)$ of which the Kochen-Specker theorem establishes the nonexistence [143].

5.3.11 The previous theorem holds for more general $\mathrm{C}^{*}$-algebras than just $\operatorname{Hilb}(H, H$ ) (for large enough Hilbert spaces $H$ ); see [74] for results on von Neumann algebras. A $C^{*}$-algebra $A$ is called simple when its closed two-sided ideals are trivial, and infinite when there is an $a \in A$ with $a^{*} a=1$ but $a a^{*} \neq$ 1 [61]. A simple infinite $C^{*}$-algebra does not admit a dispersion-free quasistate [106], whence the previous theorem holds for such $C^{*}$-algebras as well.

The rest of this section is devoted to describing the structure of the Gelfand spectrum $\underline{\Sigma}(\underline{A})$ of the Bohrification $\underline{A}$ of $A$ from the external point of view.

5.3.12 Theorem For a $C^{*}$-algebra $A$ and each $C \in \mathcal{C}(A)$, one has $\underline{L}_{A}(C)=L_{C}$. Moreover $\underline{L}_{A}(C \subseteq D): L_{C} \rightarrow L_{D}$ is a frame morphism that maps each generator $\mathrm{D}_{c}$ for $c \in C_{\mathrm{sa}}$ to the same generator for the spectrum of $D$.

Proof This follows from Lemma 5.2.14 and Proposition 5.2.18.

5.3.13 The next corollary interprets $\mathrm{D}_{a} \triangleleft U$ in our situation, showing that also the covering relation $\triangleleft$ can be computed locally. To do so, we introduce the notation $\underline{L}_{A \mid \uparrow C}$ for the restriction of the functor $\underline{L}_{A}: \mathcal{C}(A) \rightarrow$ Set to $\uparrow C \subseteq \mathcal{C}(A)$. Then $\underline{\Omega}^{\underline{\underline{L}}} \underline{\underline{A}}(C) \cong \operatorname{Sub}\left(\underline{L}_{A \mid \uparrow C}\right)$ by [164, Section II.8]. Hence, by Kripke-Joyal semantics, cf. 5.1.20, the formal variables $\mathrm{D}_{a}$ and $U$ in $C \Vdash \mathrm{D}_{a} \triangleleft U$ for $C \in \mathcal{C}(A)$ are to be instantiated with actual elements $D_{c} \in L_{C}=\underline{L}_{A}(C)$ and a subfunctor $\underline{U}: \uparrow C \rightarrow$ Set of $\underline{L}_{A \mid \uparrow C}$. Since $\triangleleft$ is a subfunctor of $\underline{L}_{A} \times \underline{\mathcal{P}}\left(\underline{L}_{A}\right)$, we can speak of $\triangleleft_{C}$ for $C \in \mathcal{C}(A)$ as the relation $\underline{L}_{\underline{A}}(C) \times \underline{\mathcal{P}}\left(\underline{L}_{\underline{A}}\right)$ induced by evaluation at $C$. 
5.3.14 Corollary The covering relation $\triangleleft$ of Theorem 5.2.23 is computed locally. That is, for $C \in \mathcal{C}(A), D_{c} \in L_{C}$ and $\underline{U} \in \operatorname{Sub}\left(\underline{L}_{A} \mid \uparrow C\right)$, the following are equivalent:

(a) $C \Vdash \mathrm{D}_{a} \triangleleft U\left(D_{c}, \underline{U}\right)$;

(b) $D_{c} \triangleleft_{C} \underline{U}(C)$;

(c) for every rational $q>0$ there is a finite $U_{0} \subseteq \underline{U}(C)$ with $D_{c-q} \leq \bigvee U_{0}$.

Proof The equivalence of (b) and (c) follows from Theorem 5.2.23. We prove the equivalence of (a) and (c). Assume, without loss of generality, that $\bigvee U_{0} \in$ $U$, so that $U_{0}$ may be replaced by $\mathrm{D}_{b}=\bigvee U_{0}$. Hence the formula $\mathrm{D}_{a} \triangleleft U$ in (a) means

$$
\forall_{q>0} \exists_{\mathrm{D}_{b} \in L_{A}} .\left(\mathrm{D}_{b} \in U \wedge \mathrm{D}_{a-q} \leq \mathrm{D}_{b}\right) .
$$

We interpret this formula step by step, as in 5.1.20. First, $C \Vdash\left(\mathrm{D}_{a} \in U\right)\left(D_{c}, \underline{U}\right)$ iff for all $D \supseteq C$ one has $D_{c} \in \underline{U}(D)$. As $\underline{U}(C) \subseteq \underline{U}(D)$, this is the case iff $D_{c} \in \underline{U}(C)$. Also one has $C \Vdash\left(\mathrm{D}_{b} \leq \mathrm{D}_{a}\right)\left(D_{c^{\prime}}, D_{c}\right)$ iff $D_{c^{\prime}} \leq D_{c}$ in $L_{C}$. Hence, $C \Vdash\left(\exists_{\mathrm{D}_{b} \in L_{A}} . \mathrm{D}_{b} \in U \wedge \mathrm{D}_{a-q} \leq \mathrm{D}_{b}\right)\left(D_{c}, \underline{U}\right)$ iff there is $D_{c^{\prime}} \in \underline{U}(C)$ with $D_{c-q} \leq D_{c^{\prime}}$. Finally, $C \Vdash\left(\forall_{q>0} \exists_{\mathrm{D}_{b} \in L_{A}} \cdot \mathrm{D}_{b} \in U \wedge \mathrm{D}_{a-q} \leq \mathrm{D}_{b}\right)\left(D_{c}, \underline{U}\right)$ iff for all $D \supseteq C$ and all rational $q>0$ there is $D_{d} \in \underline{U}(D)$ such that $D_{c-q} \leq D_{d}$, where

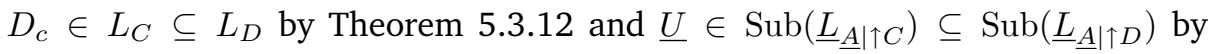
restriction. This holds at all $D \supseteq C$ iff it holds at $C$, because $\underline{U}(C) \subseteq \underline{U}(D)$, whence one can take $D_{d}=D_{c^{\prime}}$.

5.3.15 The following theorem explicitly determines the Gelfand spectrum $\underline{\Sigma}(\underline{A})$ from the external point of view. It turns out that the functor $\underline{\Sigma}(\underline{A})$ is completely determined by its value $\underline{\Sigma}(\underline{A})(\mathbb{C})$ at the least element $\mathbb{C}$ of $\mathcal{C}(A)$. Therefore, we abbreviate $\underline{\Sigma}(\underline{A})(\mathbb{C})$ by $\Sigma_{A}$, and call it the Bohrified state space of $A$.

\subsubsection{Theorem For a $C^{*}$-algebra $A$ :}

(a) At $C \in \mathcal{C}(A)$, the set $\mathcal{O}(\underline{\Sigma}(\underline{A}))(C)$ consists of the subfunctors $\underline{U} \in \operatorname{Sub}\left(\underline{L}_{A \mid \uparrow C}\right)$ satisfying $D_{d} \triangleleft_{D} \underline{U}(D) \Rightarrow D_{d} \in \underline{U}(D)$ for all $D \supseteq C$ and $D_{d} \in L_{D}$.

(b) In particular, the set $\mathcal{O}(\underline{\Sigma}(\underline{A}))(\mathbb{C})$ consists of the subfunctors $\underline{U} \in \operatorname{Sub}\left(\underline{L}_{A}\right)$ satisfying $D_{c} \triangleleft_{C} \underline{U}(C) \Rightarrow D_{c} \in \underline{U}(C)$ for all $C \in \mathcal{C}(A)$ and $D_{c} \in L_{C}$.

(c) The action $\mathcal{O}(\underline{\Sigma}(\underline{A})) \rightarrow \mathcal{O}(\underline{\Sigma}(\underline{A}))$ of $\mathcal{O}(\underline{\Sigma}(\underline{A}))$ on a morphism $C \subseteq D$ of $\mathcal{C}(A)$ is given by truncating $\underline{U}: \uparrow C \rightarrow$ Set to $\uparrow D$.

(d) The external description of $\mathcal{O}(\underline{\Sigma}(\underline{A}))$ is the frame morphism

$$
f^{-1}: \mathcal{O}(\operatorname{Alx}(\mathcal{C}(A))) \rightarrow \mathcal{O}(\underline{\Sigma}(\underline{A}))(\mathbb{C})
$$


given on basic opens $\uparrow D \in \mathcal{O}(\operatorname{Alx}(\mathcal{C}(A)))$ by

$$
f^{-1}(\uparrow D)(E)= \begin{cases}L_{E} & \text { if } E \supseteq D \\ \emptyset & \text { otherwise. }\end{cases}
$$

PRoOF By Theorem 5.2.23 (a) and (5.2), $\mathcal{O}(\underline{\Sigma}(\underline{A}))$ is the subobject of $\underline{\Omega}^{\underline{L_{A}}}$ defined by the formula $\forall_{\mathrm{D}_{a} \in L_{A}} . \mathrm{D}_{a} \triangleleft U \Rightarrow \mathrm{D}_{a} \in U$. As in 5.3.13, elements $\underline{U} \in$ $\mathcal{O}(\underline{\Sigma}(\underline{A}))(C)$ may be identified with subfunctors of $\underline{L}_{\underline{A} \mid \uparrow C}$. Hence, by Corollary 5.3.14, we have $\underline{U} \in \mathcal{O}(\underline{\Sigma}(\underline{A}))$ if and only if

$$
\forall_{D \supseteq C} \forall_{D_{d} \in L_{D}} \forall_{E \supseteq D} \cdot D_{d} \triangleleft_{E} \underline{U}(E) \Rightarrow D_{d} \in \underline{U}(E),
$$

where $D_{d}$ is regarded as an element of $L_{E}$. This is equivalent to the apparently weaker condition

$$
\forall_{D \supseteq C} \forall_{D_{d} \in L_{D}} \cdot D_{d} \triangleleft_{D} \underline{U}(D) \Rightarrow D_{d} \in \underline{U}(D),
$$

because the latter applied at $D=E$ actually implies the former condition since $D_{d} \in L_{D}$ also lies in $L_{E}$. This proves (a), (b) and (c). Part (d) follows from Example 5.1.24.

\subsection{Projections}

This section compares the quantum state spaces $\mathcal{O}(\underline{\Sigma}(\underline{A}))$ with quantum logic in the sense of Chapter 4. In a setting of operator algebras, this more traditional quantum logic of Chapter 4 concerns projections; recall from 4.1.11 that $\operatorname{Proj}(A)=\left\{p \in A \mid p^{*}=p=p \circ p\right\}$. Hence we need to specialise to $\mathrm{C}^{*}$-algebras that have enough projections. The most general such class that can easily be Bohrified turns out to consist of so-called Rickart $C^{*}$-algebras. To motivate the choice for Rickart $\mathrm{C}^{*}$-algebras, we start by recalling several types of $\mathrm{C}^{*}$-algebras and known results about their spectra.

5.4.1 Definition Let $A$ be a C*-algebra. Define $R(S)=\left\{a \in A \mid \forall_{s \in S} . s a=0\right\}$ to be the right annihilator of some subset $S \subseteq A$. Then $A$ is said to be:

(a) a von Neumann algebra if it is the dual of some Banach space [190];

(b) an $A W^{*}$-algebra if for each nonempty $S \subseteq A$ there is a $p \in \operatorname{Proj}(A)$ satisfying $R(S)=p A[138$;

(c) a Rickart $C^{*}$-algebra if for each $x \in A$ there is a $p \in \operatorname{Proj}(A)$ satisfying $R(\{x\})=p A[182]$; 
(d) a spectral $C^{*}$-algebra if for each $a \in A^{+}$and each $r, s \in(0, \infty)$ with $r<s$, there is a $p \in \operatorname{Proj}(A)$ satisfying $a p \geq r p$ and $a(1-p) \leq s(1-p)$ [203].

In all cases, the projection $p$ turns out to be unique.

5.4.2 The class of all $\mathrm{C}^{*}$-algebras is not directly relevant to traditional quantum logic in the sense of Chapter 4, as a generic $\mathrm{C}^{*}$-algebra may not have enough projections. For if $A$ is a commutative $\mathrm{C}^{*}$-algebra whose Gelfand spectrum $\Sigma(A)$ is connected, then $A$ has no projections except for 0 and 1.

5.4.3 For the other extreme, recall the Stone representation theorem [130]: any Boolean algebra is isomorphic to the lattice $\mathcal{B}(X)$ of clopen subsets of a Stone space $X$, i.e. a compact Hausdorff space that is totally disconnected, in that its only connected subsets are singletons. Equivalently, a Stone space is compact, $T_{0}$, and has a basis of clopen sets. Thus a Stone space $X$ gives rise to a Boolean algebra $\mathcal{B}(X)$ as well as to a commutative $\mathrm{C}^{*}$-algebra $C(X, \mathbb{C})$. Now, a von Neumann algebra $A$ is commutative if and only if $\operatorname{Proj}(A)$ is a Boolean algebra [180, Proposition 4.16]. In that case, the Gelfand spectrum $\Sigma(A)$ of $A$ may be identified with the Stone spectrum of $\operatorname{Proj}(A)$. In general, if $A$ is a commutative $\mathrm{C}^{*}$-algebra, then $\operatorname{Proj}(A)$ is isomorphic with the Boolean lattice $\mathcal{B}(\Sigma(A))$ of clopens in $\Sigma(A)$. If we regard $\Sigma(A)$ as consisting of characters as in 5.2.10, then this isomorphism is given by

$$
\begin{aligned}
\operatorname{Proj}(A) & \stackrel{\cong}{\rightrightarrows} \mathcal{B}(\Sigma(A)) \\
p & \mapsto \operatorname{supp}(\hat{p})=\{\sigma \in \Sigma(A) \mid \sigma(p) \neq 0\},
\end{aligned}
$$

where $\hat{p}$ is the Gelfand transform of $p$ as in 5.2.11, and the support of the Gelfand transform of $a \in A_{\mathrm{sa}}$ is $\operatorname{supp}(\hat{a})=\{\sigma \in \Sigma(A) \mid \hat{a}(\sigma) \neq 0\}=\mathcal{D}_{a}$, where support is defined as in Example 2.2.17.

5.4.4 However, the above correspondence between Boolean algebras and commutative von Neumann algebras is not bijective. If $A$ is a commutative von Neumann algebra, then $\operatorname{Proj}(A)$ is complete, so that $\Sigma(A)$ is not merely Stone but Stonean, i.e. compact, Hausdorff and extremely disconnected, in that the closure of every open set is open. (The Stone spectrum of a Boolean algebra $L$ is Stonean if and only if $L$ is complete.) But commutative von Neumann algebras do not correspond bijectively to complete Boolean algebras either, since the Gelfand spectrum of a commutative von Neumann algebra is not merely Stone but has the stronger property of being hyperstonean, in that it admits sufficiently many positive normal measures [206. Definition 1.14]. Indeed, a commutative $\mathrm{C}^{*}$-algebra $A$ is a von Neumann algebra if and only if its Gelfand spectrum (and hence the Stone spectrum of its projection lattice) is hyperstonean. 
5.4.5 Theorem A commutative $C^{*}$-algebra $A$ is:

(a) a von Neumann algebra if and only if $\Sigma(A)$ is hyperstonean [206. Section III.1];

(b) an $A W^{*}$-algebra if and only if $\Sigma(A)$ is Stonean, if and only if $\Sigma(A)$ is Stone and $\mathcal{B}(\Sigma(A))$ is complete [28. Theorem 1.7.1];

(c) a Rickart $C^{*}$-algebra if and only if $\Sigma(A)$ is Stone and $\mathcal{B}(\Sigma(A))$ is countably complete [28. Theorem 1.8.1];

(d) a spectral $C^{*}$-algebra if and only if $\Sigma(A)$ is Stone [203. Section 9.7].

5.4.6 Though spectral $C^{*}$-algebras are the most general class in Definition 5.4 .1 their projections may not form a lattice in the noncommutative case. A major advantage of Rickart $\mathrm{C}^{*}$-algebras is that their projections do, as in the following proposition. Rickart $\mathrm{C}^{*}$-algebras are also of interest for classification programmes, as follows. The class of so-called real rank zero $\mathrm{C}^{*}$-algebras has been classified using K-theory. This is a functor $K$ from CStar to graded Abelian groups. In fact, it is currently believed that real rank zero $C^{*}$-algebras are the widest class of $\mathrm{C}^{*}$-algebras for which $A \cong B$ if and only if $K(A) \cong K(B)$ [186. Section 3]. Rickart $C^{*}$-algebras are always real rank zero [31, Theorem 6.1.2].

5.4.7 Proposition Let $A$ be a Rickart $C^{*}$-algebra.

(a) If it is ordered by $p \leq q \Leftrightarrow p A \subseteq q A$, then $\operatorname{Proj}(A)$ is a countably complete lattice [28. Proposition 1.3.7 and Lemma 1.8.3].

(b) If $A$ is commutative, then it is the (norm-)closed linear span of $\operatorname{Proj}(A)[28$. Proposition 1.8.1.(3)].

(c) If $A$ is commutative, then it is monotone countably complete, i.e. each increasing bounded sequence in $A_{\mathrm{sa}}$ has a supremum in $A$ [203. Proposition 9.2.6.1].

5.4.8 Definition 5.4.1 (a) requires the so-called ultraweak or $\sigma$-weak topology, which is hard to internalise to a topos. There are constructive definitions of von Neumann algebras [66, 202], but they rely on the strong operator topology, which is hard to internalise, too. Furthermore, the latter rely on the axiom of dependent choice. Although this holds in Kripke toposes, we prefer to consider Rickart $\mathrm{C}^{*}$-algebras. All one loses in this generalisation is that the projection lattice is only countably complete instead of complete-this is not a source of tremendous worry, because countable completeness of $\operatorname{Proj}(A) \operatorname{implies}$ completeness if $A$ has a faithful representation on a separable Hilbert space. Moreover, Rickart C*-algebras can easily be Bohrified, as Theorem 5.4.11 below shows. 
5.4.9 Proposition For a commutative $C^{*}$-algebra $A$, the following are equivalent:

(a) A is Rickart;

(b) for each $a \in A$ there is a (unique) $[a=0] \in \operatorname{Proj}(A)$ such that $a[a=0]=0$, and $b=b[a=0]$ when $a b=0$;

(c) for each $a \in A_{\mathrm{sa}}$ there is a (unique) $[a>0] \in \operatorname{Proj}(A)$ such that $[a>0] a=a^{+}$ and $[a>0][-a>0]=0$.

Proof For the equivalence of (a) and (b) we refer to [28, Proposition 1.3.3]. Assuming (b) and defining $[a>0]=1-\left[a^{+}=0\right]$, we have

$$
\begin{aligned}
{[a>0] a } & =\left(1-\left[a^{+}=0\right]\right)\left(a^{+}-a^{-}\right) \\
& =a^{+}-a^{-}-a^{+}\left[a^{+}=0\right]+a^{-}\left[a^{+}=0\right] \\
& =a^{+}, \quad \quad \text { (since } a^{-} a^{+}=0, \text { so that } a^{-}\left[a^{+}=0\right]=a^{-} \text {) }
\end{aligned}
$$

and similarly $a^{-}[a>0]=a^{-}-a^{-}\left[a^{+}=0\right]=0$, whence

$$
\begin{aligned}
{[a>0][-a>0] } & =[a>0]\left(1-\left[(-a)^{+}=0\right]\right) \\
& =[a>0]-[a>0]\left[a^{-}=0\right]=0, \quad \quad \quad\left(\text { since }\left[a^{-}[a>0]=0\right)\right.
\end{aligned}
$$

establishing (c). For the converse, notice that it suffices to handle the case $a \in A^{+}$: decomposing general $a \in A$ into four positives we obtain $[a=0]$ by multiplying the four associated projections. Assuming (c) and $a \in A^{+}$, define $[a=0]=1-[a>0]$. Then $a[a=0]=(1-[a>0])=a^{+}-a[a>0]=0$. If $a b=0$ for $b \in A$, then

$$
\mathrm{D}_{b[a>0]}=\mathrm{D}_{b \wedge[a>0]}=\mathrm{D}_{b} \wedge \mathrm{D}_{[a>0]}=\mathrm{D}_{b} \wedge \mathrm{D}_{a}=\mathrm{D}_{b a}=\mathrm{D}_{0},
$$

so that $b[a<0] \preccurlyeq 0$ by 5.2.12. That is, $b[a<0] \leq n \cdot 0=0$ for some $n \in \mathbb{N}$.

5.4.10 Parallel to Proposition 5.3.3, we define $\mathcal{C}_{\mathrm{R}}(A)$ to be the collection of all commutative Rickart $\mathrm{C}^{*}$-subalgebras $C$ of $A$, and $\mathcal{T}_{\mathrm{R}}(A)=\left[\mathcal{C}_{\mathrm{R}}(A)\right.$, Set $]$. The Bohrification $\underline{A}$ of a Rickart $\mathrm{C}^{*}$-algebra $A$ is then defined by $\underline{A}(C)=C$, just as in Definition 5.3.6.

5.4.11 Theorem Let $A$ be a Rickart $C^{*}$-algebra. Then $\underline{A}$ is a commutative Rickart $C^{*}$-algebra in $\mathcal{T}_{\mathrm{R}}(A)$.

Proof By Theorem 5.3.8, we already know that $A$ is a commutative $C^{*}$-algebra in $\mathcal{T}_{\mathrm{R}}(A)$. Proposition 5.4.9 captures the property of a commutative $\mathrm{C}^{*}$-algebra being Rickart in a geometric formula. Hence, by Lemma 5.2.14, $\underline{A}$ is Rickart since every $C \in \mathcal{C}_{\mathrm{R}}(A)$ is. 
We now work towards an explicit formula for the external description of the Gelfand spectrum of the Bohrification of a Rickart C*-algebra.

5.4.12 Lemma Let $A$ be a commutative Rickart $C^{*}$-algebra, and $a, b \in A$ selfadjoint. If $a b \geq a$, then $a \preccurlyeq b$, i.e. $\mathrm{D}_{a} \leq \mathrm{D}_{b}$.

PROoF If $a \leq a b$ then certainly $a \preccurlyeq a b$. Hence $\mathrm{D}_{a} \leq \mathrm{D}_{a b}=\mathrm{D}_{a} \wedge \mathrm{D}_{b}$. In other words, $\mathrm{D}_{a} \leq \mathrm{D}_{b}$, whence $a \preccurlyeq b$.

5.4.13 Definition Recall that a function $f$ between posets satisfying $f(x) \geq$ $f(y)$ when $x \leq y$ is called antitone. A pseudocomplement on a distributive lattice $L$ is an antitone function $\neg: L \rightarrow L$ satisfying $x \wedge y=0$ iff $x \leq \neg y$. Compare Definition 4.5.1.

5.4.14 Proposition For a commutative Rickart $C^{*}$-algebra $A$, the lattice $L_{A}$ has a pseudocomplement, determined by $\neg \mathrm{D}_{a}=\mathrm{D}_{[a=0]}$ for $a \in A^{+}$.

Proof Without loss of generality, let $b \leq 1$. Then

$$
\begin{aligned}
\mathrm{D}_{a} \wedge \mathrm{D}_{b}=0 & \Longleftrightarrow \mathrm{D}_{a b}=\mathrm{D}_{0} \\
& \Longleftrightarrow a b=0 \\
& \Longleftrightarrow b[a=0]=b \\
& \Longleftrightarrow b \preccurlyeq[a=0] \quad \quad(\Leftrightarrow \text { by Proposition[5.4.9) } \\
& \Longleftrightarrow \mathrm{D}_{b} \leq \mathrm{D}_{[a=0]}=\neg \mathrm{D}_{a} .
\end{aligned}
$$

To see that $\neg$ is antitone, suppose that $\mathrm{D}_{a} \leq \mathrm{D}_{b}$. Then $a \preccurlyeq b$, so $a \leq n b$ for some $n \in \mathbb{N}$. Hence $[b=0] a \leq[b=0] b n=0$, so that $\neg \mathrm{D}_{b} \wedge \mathrm{D}_{a}=\mathrm{D}_{[b=0] a}=0$, and therefore $\neg D_{b} \leq \neg D_{a}$.

5.4.15 Lemma If $A$ is a commutative Rickart $C^{*}$-algebra, then the lattice $L_{A}$ satisfies $\mathrm{D}_{a} \leq \bigvee_{r \in \mathbb{Q}^{+}} \mathrm{D}_{[a-r>0]}$ for all $a \in A^{+}$.

Proof Since $[a>0] a=a^{+} \geq a$, Lemma 5.4 .12 gives $a \preccurlyeq[a>0]$ and therefore $\mathrm{D}_{a} \leq \mathrm{D}_{[a>0]}$. Also, for $r \in \mathbb{Q}^{+}$and $a \in A^{+}$, one has $1 \leq \frac{2}{r}((r-a) \vee a)$, whence

$$
[a-r>0] \leq \frac{2}{r}((r-a) \vee a)[a-r>0]=\frac{2}{r}(a[a-r>0]) .
$$

Lemma 5.4.12 then yields $\mathrm{D}_{[a-r>0]} \leq \mathrm{D}_{\frac{2}{r} a}=\mathrm{D}_{a}$. In total, we have $\mathrm{D}_{[a-r>0]} \leq$ $\mathrm{D}_{a} \leq \mathrm{D}_{[a>0]}$ for all $r \in \mathbb{Q}^{+}$, from which the statement follows.

The following simplifies Theorem 5.2.21 by restricting to Rickart C*-algebras. This result was not yet known, but is easily proved in Set. 
5.4.16 Theorem The Gelfand spectrum $\mathcal{O}(\Sigma(A))$ of a commutative Rickart $C^{*}$ algebra $A$ is isomorphic to the frame $\operatorname{Idl}(\operatorname{Proj}(A))$ of ideals of $\operatorname{Proj}(A)$. Hence the regularity condition may be dropped if one uses $\operatorname{Proj}(A)$ instead of $L_{A}$. Moreover, $\mathcal{O}(\Sigma(A))$ is generated by the sublattice $P_{A}=\left\{\mathrm{D}_{a} \in L_{A} \mid a \in A^{+}, \neg \neg \mathrm{D}_{a}=\mathrm{D}_{a}\right\}$ of 'clopens' of $L_{A}$, which is Boolean by construction.

Proof Since $\neg \mathrm{D}_{p}=\mathrm{D}_{1-p}$ for $p \in \operatorname{Proj}(A)$, we have $\neg \neg \mathrm{D}_{p}=\mathrm{D}_{p}$. Conversely, $\neg \neg \mathrm{D}_{a}=\mathrm{D}_{[a>0]}$, so that each element of $P_{A}$ is of the form $\mathrm{D}_{a}=\mathrm{D}_{p}$ for some $p \in \operatorname{Proj}(A)$. So $P_{A}=\left\{\mathrm{D}_{p} \mid p \in \operatorname{Proj}(A)\right\} \cong \operatorname{Proj}(A)$, since each projection $p \in \operatorname{Proj}(A)$ may be selected as the unique representative of its equivalence class $\mathrm{D}_{p}$ in $L_{A}$. By Lemma 5.4.15, we may use $\operatorname{Proj}(A)$ instead of $L_{A}$ as the generating lattice for $\mathcal{O}(\Sigma(A))$. So $\mathcal{O}(\Sigma(A))$ is the collection of regular ideals of $\operatorname{Proj}(A)$ by Theorem 5.2.21. But since $\operatorname{Proj}(A) \cong P_{A}$ is Boolean, all its ideals are regular, as $\mathrm{D}_{p} \ll \mathrm{D}_{p}$ for each $p \in \operatorname{Proj}(A)$ [130]. This establishes the statement, $\mathcal{O}(\Sigma(A)) \cong \operatorname{Idl}(\operatorname{Proj}(A))$.

We can now give a concise external description of the Gelfand spectrum of the Bohrification of a Rickart C*-algebra $A$, simplifying Theorem 5.3.16,

5.4.17 Theorem The Bohrified state space $\Sigma_{A}$ of a Rickart $C^{*}$-algebra $A$ is given by

$$
\begin{aligned}
\mathcal{O}\left(\Sigma_{A}\right) \cong\{F: \mathcal{C}(A) \rightarrow \text { Set } \mid & F(C) \in \mathcal{O}(\Sigma(C)) \text { and } \\
& \Sigma(C \subseteq D)(F(C)) \subseteq F(D) \text { if } C \subseteq D\} .
\end{aligned}
$$

It has a basis given by

$$
\mathcal{B}\left(\Sigma_{A}\right)=\{G: \mathcal{C}(A) \rightarrow \operatorname{Proj}(A) \mid G(C) \in \operatorname{Proj}(C) \text { and } G(C) \leq G(D) \text { if } C \subseteq D\} .
$$

More precisely, there is an injection $f: \mathcal{B}\left(\Sigma_{A}\right) \rightarrow \mathcal{O}\left(\Sigma_{A}\right)$ given by $f(G)(C)=$ $\operatorname{supp}(\widehat{G(C)})$, using the Gelfand transform of 5.2.11 in Set. Each $F \in \mathcal{O}\left(\Sigma_{A}\right)$ can be expressed as $F=\bigvee\left\{f(G) \mid G \in \mathcal{B}\left(\Sigma_{A}\right), f(G) \leq F\right\}$.

Proof By (the proof of) Theorem 5.4.16, one can use Proj $(C)$ instead of $\underline{L}_{A}(C)$ as a generating lattice for $\mathcal{O}(\Sigma(A))$. Translating Theorem 5.3.16(b) in these terms yields that $\mathcal{O}\left(\Sigma_{A}\right)$ consists of subfunctors $\underline{U}$ of $\underline{L}_{A}$ for which $\underline{U}(C) \in$ $\operatorname{Idl}(\operatorname{Proj}(C))$ at each $C \in \mathcal{C}(A)$. Notice that Theorem 5.3.16 holds in $\mathcal{T}_{R}(A)$ as well as in $\mathcal{T}(A)$ (by interpreting Theorem 5.2.23 in the former instead of in the latter topos). Thus we obtain a frame isomorphism $\operatorname{Idl}(\operatorname{Proj}(C)) \cong \mathcal{O}(\Sigma(C))$, and the description in the statement.

The projections $\operatorname{Proj}(A)$ form a complete orthomodular lattice (see Definition 4.3.1] for any von Neumann algebra $A$ [180]. It follows from Example 5.2.6 
that $\operatorname{Proj}(A)$ is a countably complete orthomodular lattice for any Rickart $\mathrm{C}^{*}$ algebra $A$. Using the description of the previous theorem, we can finally compare our Bohrified state space $\mathcal{O}\left(\Sigma_{A}\right)$ to the traditional "quantum $\operatorname{logic}$ " $\operatorname{Proj}(A)$. To do so, we recall an alternative characterisation of orthomodular lattices.

5.4.18 Definition A (complete) partial Boolean algebra is a family $\left(B_{i}\right)_{i \in I}$ of (complete) Boolean algebras whose operations coincide on overlaps:

- each $B_{i}$ has the same least element 0;

- $x \Rightarrow_{i} y$ if and only if $x \Rightarrow_{j} y$, when $x, y \in B_{i} \cap B_{j}$;

- if $x \Rightarrow_{i} y$ and $y \Rightarrow_{j} z$ then there is a $k \in I$ with $x \Rightarrow_{k} z$;

- $\neg_{i} x=\neg_{j} x$ when $x \in B_{i} \cap B_{j}$;

- $x \vee_{i} y=x \vee_{j} y$ when $x, y \in B_{i} \cap B_{j}$;

- if $y \Rightarrow_{i} \neg_{i} x$ for some $x, y \in B_{i}$, and $x \Rightarrow_{j} z$ and $y \Rightarrow_{k} z$, then $x, y, z \in B_{l}$ for some $l \in I$.

5.4.19 The requirements of a partial Boolean algebra imply that the amalgamation $\mathcal{A}(B)=\bigcup_{i \in I} B_{i}$ carries a well-defined structure $\vee, \wedge, 0,1, \perp$, under which it becomes an orthomodular lattice. For example, $x^{\perp}=\neg_{i} x$ for $x \in B_{i} \subseteq \mathcal{A}(B)$. Conversely, any orthomodular lattice $X$ is a partial Boolean algebra, in which $I$ is the collection of all orthogonal subsets of $\mathcal{A}(B)$, and $B_{i}$ is the sublattice of $\mathcal{A}(B)$ generated by $I$. Here, a subset $E \subseteq \mathcal{A}(B)$ is called orthogonal when pairs $(x, y)$ of different elements of $E$ are orthogonal, i.e. $x \leq y^{\perp}$. The generated sublattices $B_{i}$ are therefore automatically Boolean. If we order $I$ by inclusion, then $B_{i} \subseteq B_{j}$ when $i \leq j$. Thus there is an isomorphism between the categories of orthomodular lattices and partial Boolean algebras [63, 83, 136, 143].

5.4.20 A similar phenomenon occurs in the Heyting algebra $\mathcal{B}\left(\Sigma_{A}\right)$ of Theorem 5.4.17, when this is complete, which is the case for $\mathrm{AW}^{*}$-algebras and in particular for von Neumann algebras (provided, of course, that we require $\mathcal{C}(A)$ to consist of commutative subalgebras in the same class). Indeed, we can think of $\mathcal{B}\left(\Sigma_{A}\right)$ as an amalgamation of Boolean algebras: just as every $B_{i}$ in Definition 5.4.18 is a Boolean algebra, every $\operatorname{Proj}(C)$ in Theorem 5.4.17 is a Boolean algebra. Hence the fact that the set $I$ in Definition 5.4 .18 is replaced by the partially ordered set $\mathcal{C}(A)$ and the requirement in Theorem 5.4.17 that $G$ be monotone are responsible for making the partial Boolean algebra $\mathcal{O}\left(\Sigma_{A}\right)$ into a Heyting algebra. Indeed, this construction works more generally, as the following theorem shows. (Compare also [96] and [218], that write an orthomodular lattice as a sheaf of Boolean and distributive ones, respectively.) 
5.4.21 Theorem Let $(I, \leq)$ be a partially ordered set, and $B_{i}$ an I-indexed family of complete Boolean algebras such that $B_{i} \subseteq B_{j}$ if $i \leq j$. Then

$$
\mathcal{B}(B)=\left\{f: I \rightarrow \bigcup_{i \in I} B_{i} \mid \forall_{i \in I} . f(i) \in B_{i} \text { and } f \text { monotone }\right\}
$$

is a complete Heyting algebra, with Heyting implication

$$
(g \Rightarrow h)(i)=\bigvee\left\{x \in B_{i} \mid \forall_{j \geq i} . x \leq g(j) \Rightarrow h(j)\right\} .
$$

Proof Defining operations pointwise makes $Y$ into a frame. For example, $f \wedge g$, defined by $(f \wedge g)(i)=f(i) \wedge_{i} g(i)$, is again a well-defined monotone function whose value at $i$ lies in $B_{i}$. Hence, as in Definition 5.1.2, $\mathcal{B}(B)$ is a complete Heyting algebra by $(g \Rightarrow h)=\bigvee\{f \in Y \mid f \wedge g \leq h\}$. We now rewrite this Heyting implication:

$$
\begin{aligned}
(g \Rightarrow h)(i) & =(\bigvee\{f \in \mathcal{B}(B) \mid f \wedge g \leq h\})(i) \\
& =\bigvee\{f(i) \mid f \in \mathcal{B}(B), f \wedge g \leq h\} \\
& =\bigvee\left\{f(i) \mid f \in \mathcal{B}(B), \forall_{j \in I} . f(j) \wedge g(j) \leq h(j)\right\} \\
& =\bigvee\left\{f(i) \mid f \in \mathcal{B}(B), \forall_{j \in I} . f(j) \leq g(j) \Rightarrow h(j)\right\} \\
& \stackrel{*}{=} \bigvee\left\{x \in B_{i} \mid \forall_{j \geq i} . x \leq g(j) \Rightarrow h(j)\right\} .
\end{aligned}
$$

To finish the proof, we establish the marked equation. First, suppose that $f \in$ $\mathcal{B}(B)$ satisfies $f(j) \leq g(j) \Rightarrow h(j)$ for all $j \in I$. Take $x=f(i) \in B_{i}$. Then for all $j \geq i$ we have $x=f(i) \leq f(j) \leq g(j) \Rightarrow h(j)$. Hence the left-hand side of the marked equation is less than or equal to the right-hand side. Conversely, suppose that $x \in B_{i}$ satisfies $x \leq g(j) \Rightarrow h(j)$ for all $j \geq i$. Define $f: I \rightarrow \bigcup_{i \in I} B_{i}$ by $f(j)=x$ if $j \geq i$ and $f(j)=0$ otherwise. Then $f$ is monotone and $f(i) \in B_{i}$ for all $i \in I$, whence $f \in Y$. Moreover, $f(j) \leq g(j) \Rightarrow h(j)$ for all $j \in I$. Since $f(i) \leq x$, the right-hand side is less than or equal to the left-hand side.

5.4.22 Proposition Let $(I, \leq)$ be a partially ordered set. Let $\left(B_{i}\right)_{i \in I}$ be complete partial Boolean algebra, and suppose that $B_{i} \subseteq B_{j}$ for $i \leq j$. Then there is an injection $D: \mathcal{A}(B) \rightarrow \mathcal{B}(B)$. This injection reflects the order: if $D(x) \leq D(y)$ in $Y$, then $x \leq y$ in $X$.

Proof Define $D(x)(i)=x$ if $x \in B_{i}$ and $D(x)(i)=0$ if $x \notin B_{i}$. Suppose that $D(x)=D(y)$. Then for all $i \in I$ we have $x \in B_{i}$ iff $y \in B_{i}$. Since $x \in \mathcal{A}(B)=\bigcup_{i \in I} B_{i}$, there is some $i \in I$ with $x \in B_{i}$. For this particular $i$, we have $x=D(x)(i)=D(y)(i)=y$. Hence $D$ is injective. If $D(x) \leq D(y)$ for 
$x, y \in \mathcal{A}(B)$, pick $i \in I$ such that $x \in B_{i}$. Unless $x=0$, we have $x=D(x)(i) \leq$ $D(y)(i)=y$.

5.4.23 In the situation of the previous proposition, the Heyting algebra $\mathcal{B}(B)$ comes with its Heyting implication, whereas the orthomodular lattice $\mathcal{A}(B)$ has its Sasaki hook. Recall from 4.4.15 that the Sakaki hook satisfies the adjunction $x \leq y \Rightarrow_{\mathrm{S}} z$ iff $x \wedge y \leq z$ only for $y$ and $z$ that are compatible. This is the case if and only if $y$ and $z$ generate a Boolean subalgebra, i.e. if and only if $y, z \in B_{i}$ for some $i \in I$. In that case, the Sasaki hook $\Rightarrow_{\mathrm{S}}$ coincides with the implication $\Rightarrow$ of $B_{i}$. Hence

$$
\begin{aligned}
(D(x) \Rightarrow D(y))(i) & =\bigvee\left\{z \in B_{i} \mid \forall_{j \geq i} . z \leq D(x)(j) \Rightarrow D(y)(j)\right\} \\
& =\bigvee\left\{z \in B_{i} \mid z \leq x \Rightarrow y\right\} \\
& =\left(x \Rightarrow_{\mathrm{S}} y\right) .
\end{aligned}
$$

In particular, we find that $\Rightarrow$ and $\Rightarrow_{\mathrm{S}}$ coincide on $B_{i} \times B_{i}$ for $i \in I$; furthermore, this is precisely the case in which the Sasaki hook satisfies the defining adjunction for (Heyting) implications.

However, the canonical injection $D$ need not turn Sasaki hooks into implications in general. One finds:

$$
\begin{aligned}
D\left(x \Rightarrow_{\mathrm{S}} y\right)(i) & =\left[\begin{array}{ll}
x^{\perp} \vee(x \wedge y) & \text { if } x \Rightarrow_{\mathrm{S}} y \in B_{i} \\
0 & \text { otherwise }
\end{array}\right] \\
(D(x) \Rightarrow D(b))(i) & =\bigvee\left\{z \in B_{i} \mid \forall_{j \geq i} . z \leq\left[\begin{array}{ll}
1 & \text { if } x \notin B_{j} \\
x^{\perp} & \text { if } x \in B_{j}, y \notin B_{j} \\
x^{\perp} \vee y & \text { if } x, y \in B_{j}
\end{array}\right]\right\} .
\end{aligned}
$$

So if $x \notin B_{j}$ for any $j \geq i$, we have $D\left(x \Rightarrow_{\mathrm{S}} y\right)(i)=0 \neq 1=(D(x) \Rightarrow D(y))(i)$.

5.4.24 To end this section, we consider the so-called Bruns-Lakser completion [36, 48, 205]. The Bruns-Lakser completion of a complete lattice is a complete Heyting algebra that contains the original lattice join-densely. It is the universal in that this inclusion preserves existing distributive joins. Explicitly, the BrunsLakser completion of a lattice $L$ is the collection $\operatorname{DIdl}(L)$ of its distributive ideals. Here, an ideal $M$ is called distributive when ( $\bigvee M$ exists and) $(\bigvee M) \wedge x=$ $\bigvee_{m \in M}(m \wedge x)$ for all $x \in L$. Now consider the orthomodular lattice $X$ with the 
following Hasse diagram.

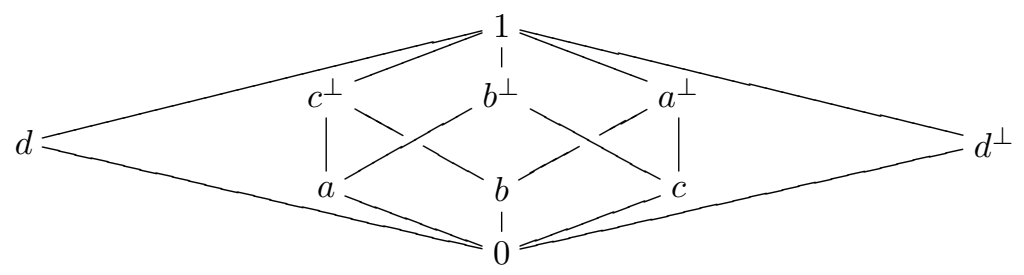

This contains precisely five Boolean algebras, namely $B_{0}=\{0,1\}$ and $B_{i}=$ $\left\{0,1, i, i^{\perp}\right\}$ for $i \in\{a, b, c, d\}$. Hence $X=\mathcal{A}(B)$ when we take $I=\{0, a, b, c, d\}$ ordered by $i<j$ iff $i=0$. The monotony requirement in $\mathcal{B}(B)$ becomes $\forall_{i \in\{a, b, c, d\}} . f(0) \leq f(i)$. If $f(0)=0 \in B_{0}$, this requirement is vacuous. But if $f(0)=1 \in B_{0}$, the other values of $f$ are already fixed. Thus one finds that $\mathcal{B}(B) \cong\left(B_{1} \times B_{2} \times B_{3} \times B_{4}\right)+1$ has 17 elements.

On the other hand, the distributive ideals of $\mathcal{A}(B)$ are given by

$$
\begin{aligned}
\operatorname{DIdl}(X) & =\left\{\left(\bigcup_{x \in A} \downarrow x\right) \cup\left(\bigcup_{y \in B} \downarrow y\right) \mid A \subseteq\left\{a, b, c, d, d^{\perp}\right\}, B \subseteq\left\{a^{\perp}, b^{\perp}, c^{\perp}\right\}\right\} \\
& -\{\emptyset\}+\{X\} .
\end{aligned}
$$

This set has 72 elements. That is, not many elements of $\mathcal{A}(B)$ are already distributive joins in $\mathcal{A}(B)$, and the Bruns-Lakser completion has to freely add a lot of elements to gain all distributive joins. In fact, in the terminology of [205],

$$
\mathcal{J}_{\text {dis }}(x)=\{S \subseteq \downarrow x \mid x \in S\},
$$

i.e. the covering relation is the trivial one, and $\operatorname{DIdl}(X)$ is the Alexandrov topology on the poset $X$. The canonical injection $D$ of Proposition 5.4.22 need not preserve the order, and hence does not satisfy the universal requirement of which the Bruns-Lakser completion is the solution. Therefore, it is unproblemetic to conclude that the construction in Theorem 5.4.21 uses more of the structure of the orthomodular lattice $\mathcal{A}(B)$ to construct a smaller Heyting algebra than the Bruns-Lakser completion.

\subsection{States and observables}

This final section considers some relationships between the external $\mathrm{C}^{*}$-algebra $A$ and its Bohrification $\underline{A}$. For example, we discuss how a state on $A$ in the operator algebraic sense gives rise to a probability integral on $\underline{A}_{\mathrm{sa}}$ within $\mathcal{T}(A)$. The latter corresponds to a suitably adapted version of a probability measure 
on $\mathcal{O}(\underline{\Sigma}(\underline{A}))$, justifying the name "Bohrified" state space. We also consider how so-called Daseinisation translates an external proposition about an observable $a \in A_{\mathrm{sa}}$ into a subobject of the Bohrified state space.

5.5.1 Definition A linear functional $\rho: A \rightarrow \mathbb{C}$ on a $C^{*}$-algebra $A$ is called positive when $\rho\left(A^{+}\right) \subseteq \mathbb{R}^{+}$. It is a state when it is positive and satisfies $\rho(1)=1$. A state $\rho$ is pure when $\rho=t \rho^{\prime}+(1-t) \rho^{\prime \prime}$ for some $t \in(0,1)$ and some states $\rho^{\prime}, \rho^{\prime \prime}$ implies $\rho^{\prime}=\rho^{\prime \prime}$. Otherwise, it is called mixed. A state is called faithful when $\rho(a)=0$ implies $a=0$ for all $a \in A^{+}$.

States are automatically Hermitian, in the sense that $\rho\left(a^{*}\right)$ is the complex conjugate of $\rho(a)$, or equivalently, $\rho(a) \in \mathbb{R}$ for $a \in A_{\text {sa }}$.

5.5.2 Example If $A=\operatorname{Hilb}(X, X)$ for some Hilbert space $X$, each unit vector $x \in X$ defines a pure state on $A$ by $\rho_{x}(a)=\langle x \mid a(x)\rangle$. Normal mixed states $\rho$ arise from countable sequences $\left(r_{i}\right)$ of numbers satisfying $0 \leq r_{i} \leq 1$ and $\sum_{i} r_{i}=1$, coupled with a family $\left(x_{i}\right)$ of $x_{i} \in X$, through $\rho(a)=\sum_{i} r_{i} \rho_{x_{i}}(a)$. This state is faithful when $\left(x_{i}\right)$ comprise an orthonormal basis of $X$ and each $r_{i}>0$.

Taking Bohr's doctrine of classical concepts seriously means accepting that two operators can only be added in a meaningful way when they commute, leading to the following notion [1, 37, 38, 165].

5.5.3 Definition A quasi-linear functional on a $\mathrm{C}^{*}$-algebra $A$ is a map $\rho: A \rightarrow \mathbb{C}$ that is linear on all commutative subalgebras and satisfies $\rho(a+i b)=\rho(a)+i \rho(b)$ for all (possibly noncommuting) $a, b \in A_{\text {sa }}$. It is called positive when $\rho\left(A^{+}\right) \subseteq$ $A^{+}$, and it is called a quasi-state when furthermore $\rho(1)=1$.

This kind of quasi-linearity determines when some property $P$ of $A$ descends to a corresponding property $\underline{P}$ for the Bohrification $\underline{A}$, as the following lemma shows. To be precise, for $P \subseteq A$, define $\underline{P} \in \operatorname{Sub}(\underline{A})$ by $\underline{P}(C)=P \cap C$. A property $P \subseteq A$ is called quasi-linear when $a, b \in P \cap A_{\mathrm{sa}}$ implies $r a+i s b \in P$ for all $r, s \in \mathbb{R}$.

5.5.4 Lemma Let $A$ be a $C^{*}$-algebra, and let $P \subseteq A$ be a quasi-linear property. Then $P=A$ if and only if $\underline{P}=\underline{A}$.

Proof One implication is trivial; for the other, suppose that $\underline{P}=\underline{A}$. For $a \in A$, denote by $C^{*}(a)$ the $C^{*}$-subalgebra generated by $a$ (and 1 ). When $a$ is selfadjoint, $C^{*}(a)$ is commutative. So $A_{\mathrm{sa}} \subseteq P$, whence by quasi-linearity of $P$ and the unique decomposition of elements in a real and imaginary part, we have $A \subseteq P$. 
5.5.5 Definition An integral on a Riesz space $R$ is a linear functional $I: R \rightarrow \mathbb{R}$ that is positive, i.e. if $f \geq 0$ then also $I(f) \geq 0$. If $R$ has a strong unit 1 (see Definition 5.2.15), then an integral $I$ satisfying $I(1)=1$ is called a probability integral. An integral $I$ is faithful when $I(f)=0$ and $f \geq 0$ imply $f=0$.

5.5.6 Example Except in the degenerate case $I(1)=0$, any integral can obviously be normalised to a probability integral. The prime example of an integral is the Riemann or Lebesgue integral on the ordered vector space $C([0,1], \mathbb{C})$. More generally, any positive linear functional on a commutative $\mathrm{C}^{*}$-algebra provides an example, states yielding probability integrals.

5.5.7 Definition Let $R$ be a Riesz space. We now define the locale $\mathcal{I}(R)$ of probability integrals on $R$. First, let $\operatorname{Int}(R)$ be the distributive lattice freely generated by symbols $\mathrm{P}_{f}$ for $f \in R$, subject to the relations

$$
\begin{aligned}
\mathrm{P}_{1} & =1, \\
\mathrm{P}_{f} \wedge \mathrm{P}_{-f} & =0, \\
\mathrm{P}_{f+g} & \leq \mathrm{P}_{f} \vee \mathrm{P}_{g}, \\
\mathrm{P}_{f} & =0
\end{aligned}
$$$$
\text { (for } f \leq 0) \text {. }
$$

This lattice generates a frame $\mathcal{O}(\mathcal{I}(R))$ by furthermore imposing the regularity condition

$$
\mathrm{P}_{f}=\bigvee\left\{\mathrm{P}_{f-q} \mid q \in \mathbb{Q}, q>0\right\} \text {. }
$$

5.5.8 Classically, points $p$ of $\mathcal{I}(R)$ correspond to probability integrals $I$ on $R$, by mapping $I$ to the point $p_{I}$ given by $p_{I}\left(\mathrm{P}_{f}\right)=1$ iff $I(f)>0$. Conversely, a point $p$ defines an integral $I_{p}=\left(\left\{q \in \mathbb{Q} \mid p \models \mathrm{P}_{f-q}\right\},\left\{r \in \mathbb{Q}|p|=\mathrm{P}_{r-f}\right\}\right)$, which is a Dedekind cut by the relations imposed on $\mathrm{P}_{(-)}$, as in Example 5.1.29. Therefore, intuitively, $\mathrm{P}_{f}=\{\rho: R \rightarrow \mathbb{R} \mid \rho(f)>0, \rho$ positive linear $\}$.

Classically, for a locally compact Hausdorff space $X$, the Riesz-Markov theorem provides a duality between integrals on a Riesz space $\{f \in C(X, \mathbb{R}) \mid$ $\operatorname{supp}(f)$ compact $\}$ and regular measures on the Borel subsets of $X$. Constructively, one uses so-called valuations, which are only defined on open subsets of $X$, instead of measures. Theorem 5.5.13 below gives a constructively valid version of the Riesz-Markov theorem. In preparation we consider a suitable constructive version of measures.

5.5.9 Classically, points of the locale $\mathbb{R}$ of Example 5.1.29 are Dedekind cuts $(L, U)$ (and $\mathcal{O}(\mathbb{R})$ is the usual Euclidean topology). We now introduce two variations on the locale $\mathbb{R}$. First, consider the locale $\mathbb{R}_{l}$ that is generated by formal 
symbols $q \in \mathbb{Q}$ subject to the following relations:

$$
q \wedge r=\min (q, r), \quad q=\bigvee\{r \mid r>q\}, \quad 1=\bigvee\{q \mid q \in \mathbb{Q}\} .
$$

Classically, its points are lower reals, and locale morphisms to $\mathbb{R}_{l}$ correspond to lower-semicontinuous real-valued functions. Restricting generators to $0 \leq q \leq 1$ yields a locale denoted $[0,1]_{l}$.

5.5.10 Secondly, let $\mathbb{R}$ be the locale defined by the very same generators $(q, r)$ and relations as in Example 5.1.29, except that we omit the fourth relation $(q, r)=\left(q, r_{1}\right) \vee\left(q_{1}, r\right)$ for $q \leq q_{1} \leq r_{1} \leq r$. The effect is that, classically, points of $\mathbb{R}$ again correspond to pairs $(L, U)$ as in Example 5.1.29. except that the lower real $L$ and the upper real $U$ need not combine into a Dedekind cut, as the 'kissing' requirement is no longer in effect. Classically, a point $(L, U)$ of $\mathbb{R} \mathbb{R}$ corresponds to a compact interval $[\sup (L), \inf (U)]$ (including the singletons $[x, x]=\{x\})$. Ordered by reverse inclusion, the topology they carry is the Scott topology [3] whose closed sets are lower sets that are closed under directed joins. Hence, each open interval $(q, r)$ in $\mathbb{R}$ (with $q=-\infty$ and $r=\infty$ allowed) corresponds to a Scott open $\{[a, b] \mid q<a \leq b<r\}$ in $\mathbb{I}$, and these form the basis of the Scott topology. Therefore, $\mathbb{I R}$ is also called the interval domain [172, 194]. One can think of it as approximations of real numbers by rational intervals, interpreting each individual interval as finitary information about the real number under scrutiny. The ordering by reverse inclusion is then explained as a smaller interval means that more information is available about the real number.

In a Kripke topos $[P$, Set $]$ over a poset $P$ with a least element, one has $\mathcal{O}(\underline{\mathbb{R}})(p)=\mathcal{O}((\uparrow p) \times \mathbb{R})$, which may be identified with the set of monotone functions from $\uparrow p$ to $\mathcal{O}(\mathbb{R})$. This follows by carefully adapting the proof of [164, Theorem VI.8.2].

5.5.11 Definition A continuous probability valuation on a locale $X$ is a monotone function $\mu: \mathcal{O}(X) \rightarrow \mathcal{O}\left([0,1]_{l}\right)$ such that $\mu(1)=1$ as well as $\mu(U)+\mu(V)=$ $\mu(U \wedge V)+\mu(U \vee V)$ and $\mu\left(\bigvee_{i} U_{i}\right)=\bigvee_{i} \mu\left(U_{i}\right)$ for a directed family $\left(U_{i}\right)$. Like integrals, continuous probability valuations organise themselves in a locale $\mathcal{V}(X)$.

5.5.12 Example If $X$ is a compact Hausdorff space, a continuous probability valuation on $\mathcal{O}(X)$ is the same thing as a regular probability measure on $X$.

5.5.13 Theorem [59] Let $R$ be an f-algebra and $\Sigma$ its spectrum. Then the locales $\mathcal{I}(R)$ and $\mathcal{V}(\Sigma)$ are isomorphic. A continuous probability valuation $\mu$ gives a probability integral by

$$
I_{\mu}(f)=\left(\sup _{\left(s_{i}\right)} \sum s_{i} \mu\left(\mathrm{D}_{f-s_{i}} \wedge \mathrm{D}_{s_{i+1}-f}\right), \inf _{\left(s_{i}\right)} \sum s_{i+1}\left(1-\mu\left(\mathrm{D}_{s_{i}-f}\right)-\mu\left(\mathrm{D}_{f-s_{i+1}}\right)\right),\right.
$$


where $\left(s_{i}\right)$ is a partition of $[a, b]$ such that $a \leq f \leq b$. Conversely, a probability integral I gives a continuous probability valuation

$$
\mu_{I}\left(\mathrm{D}_{a}\right)=\sup \left\{I\left(n a^{+} \wedge 1\right) \mid n \in \mathbb{N}\right\} .
$$

5.5.14 Corollary For a $C^{*}$-algebra $A$, the locale $\mathcal{I}(\underline{A})$ in $\mathcal{T}(A)$ of probability integrals on $\underline{A}_{\mathrm{sa}}$ is isomorphic to the locale $\mathcal{V}(\underline{\Sigma}(\underline{A}))$ in $\mathcal{T}(A)$ of continuous probability valuations on $\underline{\Sigma}(\underline{A})$.

Proof Interpret Theorem5.5.13-whose proof is constructive-in $\mathcal{T}(A)$.

5.5.15 Theorem There is a bijective correspondence between (faithful) quasi-states on a $C^{*}$-algebra $A$ and (faithful) probability integrals on $\underline{A}_{\mathrm{sa}}$.

Proof Every quasi-state $\rho$ gives a natural transformation $I_{\rho}: \underline{A}_{\mathrm{sa}} \rightarrow \underline{\mathbb{R}}$ whose component $\left(I_{\rho}\right)_{C}: C_{\mathrm{sa}} \rightarrow \mathbb{R}$ is the restriction $\rho_{\mid C_{\mathrm{sa}}}$ of $\rho$ to $C_{\mathrm{sa}} \subseteq A_{\mathrm{sa}}$. Conversely, let $I: \underline{A}_{\mathrm{sa}} \rightarrow \mathbb{R}$ be an integral. Define $\rho: A_{\mathrm{sa}} \rightarrow \mathbb{R}$ by $\rho(a)=I_{C^{*}(a)}(a)$, where $C^{*}(a)$ is the sub-C*-algebra generated by $a$. For commuting $a, b \in A_{\mathrm{sa}}$, then

$$
\begin{aligned}
\rho(a+b) & =I_{C^{*}(a+b)}(a+b) \\
& =I_{C^{*}(a, b)}(a+b) \\
& =I_{C^{*}(a, b)}(a)+I_{C^{*}(a, b)}(b) \\
& =I_{C^{*}(a)}(a)+I_{C^{*}(b)}(b) \\
& =\rho(a)+\rho(b),
\end{aligned}
$$

because $I$ is a natural transformation, $C^{*}(a) \cup C^{*}(b) \subseteq C^{*}(a, b) \supseteq C^{*}(a+b)$, and $I$ is locally linear. Moreover, $\rho$ is positive because $I$ is locally positive, by Lemma 5.5.4. Hence we have defined $\rho$ on $A_{\text {sa }}$ and may extend it to $A$ by complex linearity. It is clear that the two maps $I \mapsto \rho$ and $\rho \mapsto I$ are each other's inverse.

5.5.16 Let $\rho$ be a (quasi-)state on a $C^{*}$-algebra $A$. Then $\mu_{\rho}$ is a continuous probability valuation on $\mathcal{O}(\underline{\Sigma}(\underline{A}))$. Hence $\mu_{\rho}\left(\_\right)=1$ is a term of the internal language of $\mathcal{T}(A)$ with one free variable of type $\mathcal{O}(\underline{\Sigma}(\underline{A}))$. Its interpretation $\llbracket \mu_{\rho}\left({ }_{-}\right)=1 \rrbracket$ defines a subobject of $\mathcal{O}(\underline{\Sigma}(\underline{A}))$, or equivalently, a morphism $[\rho]: \mathcal{O}(\underline{\Sigma}(\underline{A})) \rightarrow \underline{\Omega}$. This transfers the classical description of states as described in Chapter 1 to the quantum situation.

For Rickart $C^{*}$-algebras, we can make Theorem 5.5.15 a bit more precise.

5.5.17 Definition (a) A probability measure on a countably complete orthomodular lattice $X$ is a function $\mu: X \rightarrow[0,1]_{l}$ that on any countably complete Boolean sublattice of $X$ restricts to a probability measure (in the traditional sense). 
(b) A probability valuation on an orthomodular lattice $X$ is a function $\mu: X \rightarrow$ $[0,1]_{l}$ such that $\mu(0)=0, \mu(1)=1, \mu(x)+\mu(y)=\mu(x \wedge y)+\mu(x \vee y)$, and if $x \leq y$ then $\mu(x) \leq \mu(y)$.

5.5.18 Lemma Let $\mu$ be a probability valuation on a Boolean algebra $X$. Then $\mu(x)$ is a Dedekind cut for any $x \in X$.

Proof Since $X$ is Boolean, we have $\mu(\neg x)=1-\mu(x)$. Let $q, r \in \mathbb{Q}$, and suppose that $q<r$. We have to prove that $q<\mu(x)$ or $\mu(x) \leq r$. As the inequalities concern rationals, it suffices to prove that $q<\mu(x)$ or $1-r<1-\mu(x)=\mu(\neg x)$. This follows from $1-(r-q)<1=\mu(1)=\mu(x \vee \neg x)$ and $q-r<0=\mu(0)=$ $\mu(x \wedge \neg x)$.

The following theorem relates Definition 5.5.11 and Definition 5.5.17 Definition 5.5.11 will be applied to the Gelfand spectrum $\underline{\Sigma}(\underline{A})$ of the Bohrification of a Rickart $C^{*}$-algebra $A$. Part (a) of Definition 5.5.17 will be applied to $\operatorname{Proj}(A)$ in Set for a Rickart C*-algebra $A$, and part (b) will be applied to the lattice $\underline{P}_{A}$ of Theorem 5.4.16 in $\mathcal{T}(A)$.

5.5.19 Theorem For a Rickart $C^{*}$-algebra $A$, there is a bijective correspondence between:

(a) quasi-states on $A$;

(b) probability measures on $\operatorname{Proj}(A)$;

(c) probability valuations on $\underline{P}_{A}$;

(d) continuous probability valuations on $\underline{\Sigma}(\underline{A})$.

PROOF The correspondence between (a) and (d) is Theorem 5.5.15 The correspondence between (c) and (d) follows from Theorem 5.4.16 and the observation that valuations on a compact regular frame are determined by their behaviour on a generating lattice [59, Section 3.3]; indeed, if a frame $\mathcal{O}(X)$ is generated by $L$, then a probability measure $\mu$ on $L$ yields a continuous probability valuation $\nu$ on $\mathcal{O}(X)$ by $\nu(U)=\sup \{\mu(u) \mid u \in U\}$, where $U \subseteq L$ is regarded as an element of $\mathcal{O}(X)$. Finally, we turn to the correspondence between (b) and (c). Since $\mathbb{R}$ in $\mathcal{T}(A)$ is the constant functor $C \mapsto \mathbb{R}$ (as opposed to $\mathbb{R}_{l}$ ), according to the previous lemma a probability valuation $\mu: \underline{\operatorname{Idl}}(\operatorname{Proj}(\underline{A})) \rightarrow[0,1]_{l}$ is defined by its components $\mu_{C}$ : $\operatorname{Proj}(C) \rightarrow[0,1]$. By naturality, for $p \in \overline{\operatorname{Proj}(C)}$, the real number $\mu_{C}(p)$ is independent of $C$, from which the correspondence between (b) and (c) follows immediately. 
5.5.20 We now turn to internalising an elementary proposition $a \in(q, r)$ concerning an observable $a \in A_{\mathrm{sa}}$ and rationals $q, r \in \mathbb{Q}$ with $q<r$. If $A$ were commutative, then $a$ would have a Gelfand transform $\hat{a}: \Sigma(A) \rightarrow \mathbb{R}$, and we could just internalise $\hat{a}^{-1}(q, r) \subseteq \Sigma(A)$ directly. For noncommutative $A$, there can be contexts $C \in \mathcal{C}(A)$ that do not contain $a$, and therefore the best we can do is approximate. Our strategy is to replace the reals $\mathbb{R}$ by the interval domain $\mathbb{R}$ of 5.5.10. We will construct a locale morphism $\underline{\delta}(a): \underline{\Sigma}(\underline{A}) \rightarrow \underline{\mathbb{R}}$, called the Daseinisation of $a \in A_{\mathrm{sa}}$-this terminology stems from [75], but the morphism is quite different from the implementation in that article. The elementary proposition $a \in(q, r)$ is then internalised as the composite morphism

$$
\underline{1} \stackrel{(q, r)}{\longrightarrow} \mathcal{O}(\underline{\mathbb{I}}) \stackrel{\underline{\delta}(a)^{-1}}{\longrightarrow} \mathcal{O}(\underline{\Sigma}(\underline{A})),
$$

where $(q, r)$ maps into the monotone function with constant value $\downarrow(q, r)$. (As in 5.5.10, $(q, r)$ is seen as an element of the generating semilattice, whereas $\downarrow(q, r)$ is its image in the frame $\mathcal{O}(\mathbb{I R})$ under the canonical inclusion of Proposition 5.1.11,)

5.5.21 The interval domain $\mathcal{O}(\mathbb{R})$ of 5.5 .10 can be constructed as $\mathcal{F}(\mathbb{Q} \times<$ $\mathbb{Q}, 4)$, as in Definition 5.1.10 [172]. The pertinent meet-semilattice $\mathbb{Q} \times<\mathbb{Q}$ consists of pairs $(q, r) \in \mathbb{Q} \times \mathbb{Q}$ with $q<r$, ordered by inclusion (i.e. $(q, r) \leq$ $\left(q^{\prime}, r^{\prime}\right)$ iff $q^{\prime} \leq q$ and $\left.r \leq r^{\prime}\right)$, with a least element 0 added. The covering relation $\triangleleft$ is defined by $0 \triangleleft U$ for all $U$, and $(q, r) \triangleleft U$ iff for all rational $q^{\prime}, r^{\prime}$ with $q<q^{\prime}<r^{\prime}<r$ there exists $\left(q^{\prime \prime}, r^{\prime \prime}\right) \in U$ with $\left(q^{\prime}, r^{\prime}\right) \leq\left(q^{\prime \prime}, r^{\prime \prime}\right)$. In particular, we may regard $\mathcal{O}(\mathbb{R})$ as a subobject of $\mathbb{Q} \times<\mathbb{Q}$. As in 5.3.13.

$$
\mathcal{O}(\underline{\mathbb{R}})(\mathbb{C}) \cong\left\{\underline{F} \in \operatorname{Sub}(\underline{\mathbb{Q} \times} \times \mathbb{Q}) \mid \forall_{C \in \mathcal{C}(A)} \cdot \underline{F}(C) \in \mathcal{O}(\mathbb{I R})\right\} .
$$

5.5.22 Lemma For a $C^{*}$-algebra $A$ and a fixed element $a \in A_{\mathrm{sa}}$, the components $\underline{d}(a)_{C}: \mathbb{Q} \times<\mathbb{Q} \rightarrow \operatorname{Sub}\left(\underline{L}_{A} \mid \uparrow C\right)$ given by

$$
\begin{aligned}
\underline{d}(a)_{C}^{*}(q, r)(D) & =\left\{\mathrm{D}_{f-q} \wedge \mathrm{D}_{r-g} \mid f, g \in D_{\mathrm{sa}}, f \leq a \leq g\right\} \\
\underline{d}(a)_{C}^{*}(0)(D) & =\left\{\mathrm{D}_{0}\right\}
\end{aligned}
$$

form a morphism $\underline{d}(a)^{*}: \underline{\mathbb{Q}} \times<\mathbb{Q} \rightarrow \underline{\Omega^{\underline{L}} \underline{\underline{A}}}$ in $\mathcal{T}(A)$ via 5.3.13. This morphism is a continuous map $\left.\left(\underline{L}_{\underline{A}}, \unlhd\right) \stackrel{(\underline{\mathbb{Q} \times}}{<} \mathbb{\mathbb { Q }}, \unlhd\right)$ in the sense of Definition 5.1 .12

Notice that since $\mathbb{Q} \times<\mathbb{Q}(C)=\mathbb{Q} \times<\mathbb{Q}$ for any $C \in \mathcal{C}(A)$, the natural transformation $\underline{d}(a)$ is completely determined by its component at $\mathbb{C} \in \mathcal{C}(A)$.

ProOF We verify that the map defined in the statement satisfies the conditions of Definition 5.1.12. 


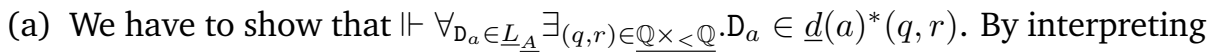
via 5.1.20, we therefore have to prove: for all $C \in \mathcal{C}(A)$ and $\mathrm{D}_{c} \in L_{C}$ there are $(q, r) \in \mathbb{Q} \times<\mathbb{Q}$ and $f, g \in C_{\mathrm{sa}}$ such that $f \leq a \leq g$ and $\mathrm{D}_{c}=\mathrm{D}_{f-q} \wedge \mathrm{D}_{r-g}$. Equivalently, we have to find $(q, r) \in \mathbb{Q} \times<\mathbb{Q}$ and $f, g \in C_{\text {sa }}$ such that $f+q \leq a \leq r+g$ and $\mathrm{D}_{c}=\mathrm{D}_{f} \wedge \mathrm{D}_{-g}$. Choosing $f=c, g=-c, q=-\|c\|-\|a\|$ and $r=\|c\|+\|a\|$ does the job, since $\mathrm{D}_{c}=\mathrm{D}_{c} \wedge \mathrm{D}_{c}$ and

$$
f+q=c-\|c\|-\|a\| \leq-\|a\| \leq a \leq\|a\| \leq\|c\|+\|a\|-c=r+g .
$$

(b) We have to show that

$$
\begin{array}{r}
\Vdash \forall(q, r),\left(q^{\prime}, r^{\prime}\right) \in \underline{\mathbb{Q} \times<\mathbb{Q}}^{\forall} u, v \in \underline{\underline{L}}_{\underline{A}} \cdot u \in \underline{d}(a)^{*}(q, r) \wedge v \in \underline{d}(a)^{*}\left(q^{\prime}, r^{\prime}\right) \\
\Rightarrow u \wedge v \triangleleft \underline{d}(a)^{*}\left((q, r) \wedge\left(q^{\prime}, r^{\prime}\right)\right) .
\end{array}
$$

Going through the motions of 5.1.20, that means we have to prove: for all $(q, r),\left(q^{\prime}, r^{\prime}\right) \in \mathbb{Q} \times<\mathbb{Q}, C \subseteq D \in \mathcal{C}(A)$ and $f, f^{\prime}, g, g^{\prime} \in C_{\text {sa }}$, if $\left(q^{\prime \prime}, r^{\prime \prime}\right)=$ $(q, r) \wedge\left(q^{\prime}, r^{\prime}\right) \neq 0, f \leq a \leq g$ and $f^{\prime} \leq a \leq g^{\prime}$, then

$$
\begin{aligned}
& \mathrm{D}_{f-q} \wedge \mathrm{D}_{r-g} \wedge \mathrm{D}_{f^{\prime}-q^{\prime}} \wedge \mathrm{D}_{r^{\prime}-g^{\prime}} \\
& \triangleleft\left\{\mathrm{D}_{f^{\prime \prime}-q^{\prime \prime}} \wedge \mathrm{D}_{r^{\prime \prime}-g^{\prime \prime}} \mid f^{\prime \prime}, g^{\prime \prime} \in D_{\mathrm{sa}}, f^{\prime \prime} \leq a \leq g^{\prime \prime}\right\} .
\end{aligned}
$$

We distinguish the possible cases of $\left(q^{\prime \prime}, r^{\prime \prime}\right)$ (which distinction is constructively valid since it concerns rationals). For example, if $\left(q^{\prime \prime}, r^{\prime \prime}\right)=\left(q, r^{\prime}\right)$, then $q \leq q^{\prime} \leq r \leq r^{\prime}$. So $\mathrm{D}_{f-q} \wedge \mathrm{D}_{r^{\prime}-g^{\prime}}=\mathrm{D}_{f^{\prime \prime}-q^{\prime \prime}} \wedge \mathrm{D}_{r^{\prime \prime}-g^{\prime \prime}}$ for $f^{\prime \prime}=f$, $g^{\prime \prime}=g^{\prime}, q^{\prime \prime}=q$ and $r^{\prime \prime}=r^{\prime}$, whence the statement holds by (a) and (c) of Definition 5.1.8. The other cases are analogous.

(c) We have to show that

$$
\Vdash \forall_{(q, r) \in \underline{\mathbb{Q} \times<\mathbb{Q}}} \forall_{U \in \underline{\mathcal{P}(\mathbb{Q} \times<\mathbb{Q})}} .(q, r) \triangleleft U \Rightarrow \underline{d}(a)^{*}(q, r) \triangleleft \bigcup_{\left(q^{\prime}, r^{\prime}\right) \in U} \underline{d}(a)^{*}\left(q^{\prime}, r^{\prime}\right) .
$$

By 5.1.20, we therefore have to prove: for all $(q, r) \in \mathbb{Q} \times<\mathbb{Q}, U \subseteq U^{\prime} \subseteq$ $\mathbb{Q} \times<\mathbb{Q}, D \in \mathcal{C}(A)$ and $f, g \in D_{\mathrm{sa}}$, if $(q, r) \triangleleft U$ and $f \leq a \leq g$, then

$$
\mathrm{D}_{f-q} \wedge \mathrm{D}_{r-g} \triangleleft\left\{\mathrm{D}_{f^{\prime}-q^{\prime}} \wedge \mathrm{D}_{r^{\prime}-g^{\prime}} \mid\left(q^{\prime}, r^{\prime}\right) \in U^{\prime}, f^{\prime}, g^{\prime} \in D_{\mathrm{sa}}, f^{\prime} \leq a \leq g^{\prime}\right\} .
$$

To establish this, it suffices to show $\mathrm{D}_{f-q} \wedge \mathrm{D}_{r-g} \triangleleft\left\{\mathrm{D}_{f-q^{\prime}} \wedge \mathrm{D}_{r^{\prime}-g} \mid\left(q^{\prime}, r^{\prime}\right) \in U\right\}$ when $(q, r) \triangleleft U$. Let $s \in \mathbb{Q}$ satisfy $0<s$. Then one has $(q, r-s)<(q, r)$. Since $(q, r) \triangleleft U$, 5.5.21 yields a $\left(q^{\prime \prime}, r^{\prime \prime}\right) \in U$ such that $(q, r-s) \leq\left(q^{\prime \prime}, r^{\prime \prime}\right)$, and so $r-s \leq r^{\prime \prime}$. Taking $U_{0}=\left\{\left(q^{\prime \prime}, r^{\prime \prime}\right)\right\}$, one has $r-g-s \leq r^{\prime \prime}-$ $g$ and therefore $\mathrm{D}_{r-g-s} \leq \mathrm{D}_{r^{\prime \prime}-g}=\bigvee U_{0}$. So, by Corollary 5.3.14, we 
have $\mathrm{D}_{r-g} \triangleleft\left\{\mathrm{D}_{r^{\prime}-g} \mid\left(q^{\prime}, r^{\prime}\right) \in U\right\}$. Similarly, one finds $\mathrm{D}_{f-q} \triangleleft\left\{\mathrm{D}_{f-q^{\prime}} \mid\right.$ $\left.\left(q^{\prime}, r^{\prime}\right) \in U\right\}$. Finally, $\mathrm{D}_{f-q} \wedge \mathrm{D}_{r-g} \triangleleft\left\{\mathrm{D}_{f-q^{\prime}} \wedge \mathrm{D}_{r^{\prime}-g} \mid\left(q^{\prime}, r^{\prime}\right) \in U\right\}$ by Definition $5.1 .8(\mathrm{~d})$.

5.5.23 Definition Let $A$ be a $C^{*}$-algebra. The Daseinisation of $a \in A_{\mathrm{sa}}$ is the locale morphism $\underline{\delta}(a): \underline{\Sigma}(\underline{A}) \rightarrow \underline{\mathbb{R}}$, whose associated frame morphism $\underline{\delta}(a)^{-1}$ is given by $\mathcal{F}\left(\underline{d}(a)^{*}\right)$, where $\mathcal{F}$ is the functor of Proposition 5.1.13, and $\underline{d}(a)$ comes from Lemma 5.5.22.

5.5.24 Example The locale $\underline{\Sigma}(\underline{A})$ is described externally by its value at $\mathbb{C} \in$ $\mathcal{C}(A)$, see Theorem 5.3.16. The component at $\mathbb{C}$ of the Daseinisation $\underline{\delta}(a)$ is given by

$$
\underline{\delta}(a)_{\mathbb{C}}^{-1}(q, r)(C)=\left\{\mathrm{D}_{f-q} \wedge \mathrm{D}_{r-g} \mid f, g \in C_{\mathrm{sa}}, f \leq a \leq g\right\} .
$$

Now suppose that $A$ is commutative. Then, classically, $\mathrm{D}_{a}=\{\rho \in \Sigma(A) \mid \rho(a)>$ $0\}$ as in 5.2.10. Hence $\mathrm{D}_{f-r}=\{\rho \in \Sigma(A) \mid \rho(f)>r\}$, so that

$$
\begin{aligned}
\underline{\delta}(a)_{\mathbb{C}}^{-1}(q, r)(C) & =\bigcup_{\substack{f, g \in C_{\mathrm{sa}} \\
f \leq a \leq g}}\{\rho \in \Sigma(A) \mid \rho(f)>q \text { and } \rho(g)<r\} \\
& =\left\{\rho \in \Sigma(A) \mid \exists_{f \leq a} \cdot q<\rho(f)<r \text { and } \exists_{g \geq a} . q<\rho(g)<r\right\} \\
& =\{\rho \in \Sigma(A) \mid q<\rho(a)<r\} \\
& =\hat{a}^{-1}(q, r) .
\end{aligned}
$$

5.5.25 Proposition The map $\underline{\delta}: A_{\mathrm{sa}} \rightarrow C(\underline{\Sigma}(\underline{A}), \underline{\mathbb{R}})$ is injective. Moreover $a \leq b$ if and only if $\underline{\delta}(a) \leq \underline{\delta}(b)$.

Proof Suppose that $\underline{\delta}(a)=\underline{\delta}(b)$. Then for all $C \in \mathcal{C}(A)$, the sets $L_{a}(C)=$ $\left\{f \in C_{\mathrm{sa}} \mid f \leq a\right\}$ and $U_{a}(C)=\left\{g \in C_{\mathrm{sa}} \mid a \leq g\right\}$ must coincide with $L_{b}(C)$ and $U_{b}(C)$, respectively. Imposing these equalities at $C=C^{*}(a)$ and at $C=$ $C^{*}(b)$ yields $a=b$. The order in $A_{\mathrm{sa}}$ is clearly preserved by $\underline{\delta}$, whereas the converse implication can be shown by the same method as the first claim of the proposition. 


\section{Bibliography}

[1] Johan F. Aarnes. Quasi-states on C*-algebras. Transactions of the American Mathematical Society, 149:601-625, 1970.

[2] Samson Abramsky and Bob Coecke. A categorical semantics of quantum protocols. In Logic in Computer Science 19, pages 415-425. IEEE Computer Society, 2004.

[3] Samson Abramsky and Achim Jung. Domain theory. In Handbook of Logic in Computer Science Volume 3, pages 1-168. Oxford University Press, 1994.

[4] Samson Abramsky and Duško Pavlović. Specifying processes. In Proceedings of the International Symposium on Category Theory In Computer Science, volume 1290 of Springer Lecture Notes in Computer Science, pages 147-158. Springer, 1997.

[5] Samsom Abransky and Steven Vickers. Quantales, observational logic and process semantics. Mathematical Structures in Computer Science, 3:161-227, 1993.

[6] Peter Aczel. Aspects of general topology in constructive set theory. Annals of Pure and Applied Logic, 137:3-29, 2006.

[7] Ichiro Amemiya and Huzihiro Araki. A remark on Piron's paper. Publications of the Research Institute for Mathematical Sciences A, 2(3):423-427, 1966.

[8] Frank W. Anderson and Kent R. Fuller. Rings and categories of modules. Springer, 1974.

[9] Robert B. Ash. Abstract Algebra: the basic graduate year. Published online at http://www.math.uiuc.edu/ r-ash 2000.

[10] Jean-Pierre Aubin. Applied Functional Analysis. Wiley Interscience, 2nd edition, 2000.

[11] John C. Baez. Higher-dimensional algebra II: 2-Hilbert spaces. Advances in Mathematics, 127:125-189, 1997.

[12] John C. Baez and James Dolan. Higher-dimensional algebra and topological quantum field theory. Journal of Mathematical Physics, 36(11):6073-6105, 1995.

[13] John C. Baez and James Dolan. Higher-dimensional algebra III: $n$-categories and the algebra of opetopes. Advances in Mathematics, 135(145-206), 1998. 
[14] Alexandru Baltag and Sonja Smets. LQP: The dynamic logic of quantum information. Mathematical Structures in Computer Science, 16(3):491-525, 2006.

[15] Alexandru Baltag and Sonja Smets. A dynamic-logical perspective on quantum behavior. Studia Logica, 89:185-209, 2008.

[16] Bernhard Banaschewski and Christopher J. Mulvey. The spectral theory of commutative C*-algebras: the constructive Gelfand-Mazur theorem. Quaestiones Mathematicae, 23(4):465-488, 2000.

[17] Bernhard Banaschewski and Christopher J. Mulvey. The spectral theory of commutative $\mathrm{C}^{*}$-algebras: the constructive spectrum. Quaestiones Mathematicae, 23(4):425-464, 2000.

[18] Bernhard Banaschewski and Christopher J. Mulvey. A globalisation of the Gelfand duality theorem. Annals of Pure and Applied Logic, 137:62-103, 2006.

[19] Michael Barr. Exact categories. In Exact Categories and Categories of Sheaves, number 236 in Lecture Notes in Mathematics, pages 1-120. Springer, 1971.

[20] Michael Barr. *autonomous categories, volume 752 of Lecture Notes in Mathematics. Springer, 1979.

[21] Michael Barr. Algebraically compact functors. Journal of Pure and Applied Algebra, 82:211-231, 1992.

[22] Michael Barr. *-autonomous categories: once more around the track. Theory and Applications of Categories, 6:5-24, 1999.

[23] Michael Barr and Charles Wells. Toposes, Triples and Theories. Springer, 1985.

[24] Giulia Battilotti and Giovanni Sambin. Pretopologies and uniform presentation of sup-lattices, quantales and frames. Annals of Pure and Applied Logic, 137:30-61, 2006.

[25] John L. Bell. Toposes and Local Set Theories. An Introduction. Number 14 in Oxford Logic Guides. Oxford University Press, 1988.

[26] Jean Bénabou. Introduction to bicategories. In Reports of the Midwest Category Seminar, volume 47 of Lecture Notes in Mathematics, pages 1-77. Springer, 1967.

[27] Charles H. Bennett and Giles Brassard. Quantum cryptography: Public key distribution and coin tossing. In Computers, Systems and Signal Processing, pages 175-179. IEEE Computer Society, 1984.

[28] Sterling K. Berberian. Baer *-rings. Springer, 1972.

[29] Garrett Birkhoff. Lattice Theory. American Mathematical Society, 1948.

[30] Garrett Birkhoff and John von Neumann. The logic of quantum mechanics. Annals of Mathematics, 37:823-843, 1936.

[31] Bruce Blackadar. Projections in $C^{*}$-algebras. In $C^{*}$-algebras: a fifty year celebration 1943-1993, pages 131-149. Providence, 1993. 
[32] Niels Bohr. Discussion with Einstein on epistemological problems in atomic physics. In Albert Einstein: Philosopher-Scientist, pages 201-241. La Salle: Open Court, 1949.

[33] Francis Borceux. Handbook of Categorical Algebra 1: Basic Category Theory. Encyclopedia of Mathematics and its Applications 50. Cambridge University Press, 1994.

[34] Francis Borceux. Handbook of Categorical Algebra 2: Categories and Structures. Encyclopedia of Mathematics and its Applications 51. Cambridge University Press, 1994.

[35] Francis Borceux. Handbook of Categorical Algebra 3: Categories of Sheaves. Encyclopedia of Mathematics and its Applications 52. Cambridge University Press, 1994.

[36] Günter Bruns and Harry Lakser. Injective hulls of semilattices. Canadian Mathematical Bulletin, 13:115-118, 1970.

[37] Leslie J. Bunce and J. D. Maitland Wright. The Mackey-Gleason problem for vector measures on projections in von Neumann algebras. Journal of the London Mathematical Society 2, 49(1):133-149, 1994.

[38] Leslie J. Bunce and J. D. Maitland Wright. The quasi-linearity problem for C* algebras. Pacific Journal of Mathematics, 172(1):41-47, 1996.

[39] Jeremy Butterfield, John Hamilton, and Christopher J. Isham. A topos perspective on the Kochen-Specker theorem: III. Von Neumann algebras as the base category. International Journal of Theoretical Physics, 39(6):1413-1436, 2000.

[40] Jeremy Butterfield and Christopher J. Isham. A topos perspective on the KochenSpecker theorem: I. Quantum states as generalized valuations. International Journal of Theoretical Physics, 37(11):2669-2733, 1998.

[41] Jeremy Butterfield and Christopher J. Isham. A topos perspective on the KochenSpecker theorem: II. Conceptual aspects and classical analogues. International Journal of Theoretical Physics, 38(3):827-859, 1999.

[42] Jeremy Butterfield and Christopher J. Isham. A topos perspective on the KochenSpecker theorem: IV. Interval valuations. International Journal of Theoretical Physics, 41(4):613-639, 2002.

[43] Carsten Butz. Regular categories and regular logic. BRICS Lecture Series LS-98-2, 1998.

[44] Aurelio Carboni, Stefano Kasangian, and Ross Street. Bicategories of spans and relations. Journal of Pure and Applied Algebra, 33:259-267, 1984.

[45] Martijn Caspers, Chris Heunen, Nicolaas P. Landsman, and Bas Spitters. Intuitionistic quantum logic of an $n$-level system. Foundations of Physics, 39(7):731-759, 2009. 
[46] Jan Cederquist and Thierry Coquand. Entailment relations and distributive lattices. In Logic Colloquium '98 (Prague), volume 13 of Lecture Notes in Logic, pages 127-139. Association for Symbolic Logic, 2000.

[47] Chen C. Chang and H. Jerome Keisler. Model Theory. North-Holland, third edition, 1990.

[48] Bob Coecke. Quantum logic in intuitionistic perspective. Studia Logica, 70:411440, 2002.

[49] Bob Coecke and Ross Duncan. Interacting quantum observables. In International Colloquium on Automata, Languages and Programming, volume 5126 of Lecture Notes in Computer Science, pages 298-310. Springer, 2008.

[50] Bob Coecke and Éric O. Paquette. POVMs and Naimark's theorem without sums. In Quantum Programming Languages, volume 210 of Electronic Notes in Theoretical Computer Science, pages 15-31. Elsevier, 2006.

[51] Bob Coecke, Éric O. Paquette, and Simon Perdrix. Bases in diagrammatic quantum protocols. In Mathematical Foundations of Programming Semantics 24, volume 218 of Electronic Notes in Theoretical Computer Science, pages 131-152. Elsevier, 2008.

[52] Bob Coecke and Duško Pavlović. Quantum measurements without sums. In Mathematics of Quantum Computing and Technology. Taylor and Francis, 2007.

[53] Bob Coecke, Duško Pavlović, and Jamie Vicary. A new description of orthogonal bases. Mathematical Structures in Computer Science, 2009.

[54] Bob Coecke and Sonja Smets. The Sasaki hook is not a [static] implicative connective but induces a backward [in time] dynamic one that assigns causes. International Journal of Theoretical Physics, 43:1705-1736, 2004.

[55] Paul M. Cohn. Skew fields. Encyclopedia of Mathematics and its Applications 57. Cambridge University Press, 1995.

[56] Alain Connes. Noncommutative Geometry. Academic Press, 1994.

[57] Thierry Coquand. About Stone's notion of spectrum. Journal of Pure and Applied Algebra, 197:141-158, 2005.

[58] Thierry Coquand and Bas Spitters. Constructive Gelfand duality for C*-algebras. Mathematical Proceedings of the Cambridge Philosophical Society, 2009. To appear.

[59] Thierry Coquand and Bas Spitters. Integrals and valuations. Journal of Logic and Analysis, 1(3):1-22, 2009.

[60] Thiery Coquand and Bas Spitters. Formal topology and constructive mathematics: the Gelfand and Stone-Yosida representation theorems. Journal of Universal Computer Science, 11(12):1932-1944, 2005.

[61] Joachim Cuntz. The structure of multiplication and addition in simple C*-algebras. Mathematica Scandinavica, 40:215-233, 1977. 
[62] Maria L. Dalla Chiara and Roberto Giuntini. Quantum logics. In Handbook of Philosophical Logic, volume VI, pages 129-228. Kluwer, 2002.

[63] Maria L. Dalla Chiara, Roberto Giuntini, and Richard Greechie. Reasoning in quantum theory: sharp and unsharp quantum logics. Springer, 2004.

[64] Brian A. Davey and Hilary A. Priestley. Introduction to Lattices and Order. Cambridge University Press, second edition, 2002.

[65] Brian J. Day. Note on compact closed categories. Journal of the Australian Mathematical Society, Series A 24(3):309-311, 1977.

[66] Luminiţa (Vîţă) Dediu and Douglas Bridges. Embedding a linear subset of $B(H)$ in the dual of its predual. In Reuniting the Antipodes-Constructive and Nonstandard Views of the Continuum, pages 55-61. Kluwer, 2001.

[67] Pierre Deligne. Catégories tannakiennes. In The Grothendieck Festschrift, volume 2, pages 111-195. Birkhauser, 1990.

[68] Ellie D'Hondt and Prakash Panangaden. Quantum weakest preconditions. Mathematical Structures in Computer Science, 16(3):429-451, 2006.

[69] Dennis Dieks. Communication by EPR devices. Physics Letters A, 92(6):271-272, 1982.

[70] Whitfield Diffie and Martin E. Hellman. New directions in cryptography. IEEE Transactions on Information Theory, IT-22(6):644-654, 1976.

[71] Jacques Dixmier. C*-algebras. North-Holland, 1977.

[72] Sergio Doplicher and John E. Roberts. A new duality theory for compact groups. Inventiones Mathematicae, 98:157-218, 1989.

[73] Robert S. Doran and Victor A. Belfi. Characterizations of $C^{*}$-algebras: the GelfandNaimark theorems. Number 101 in Pure and Applied Mathematics. Marcel Dekker, Inc., 1986.

[74] Andreas Döring. Kochen-Specker theorem for Von Neumann algebras. International Journal of Theoretical Physics, 44(2):139-160, 2005.

[75] Andreas Döring and Christopher J. Isham. A topos foundation for theories of physics. I-IV. Journal of Mathematical Physics, 49:053515-053518, 2008.

[76] Andreas Döring and Christopher J. Isham. 'What is a thing?': Topos theory in the foundations of physics. In New Structures for Physics, Lecture Notes in Physics. Springer, 2009.

[77] Ross Duncan. Types for Quantum Computing. PhD thesis, Oxford University Computer Laboratory, 2006.

[78] Beno Eckmann and Peter Hilton. Group-like structures in categories. Mathematische Annalen, 145:227-255, 1962. 
[79] Albert Einstein, Boris Podolsky, and Nathan Rosen. Can quantum-mechanical description of physical reality be considered complete? Physical Review, 47:777-780, 1935.

[80] Artur K. Ekert. Quantum cryptography based on Bell's theorem. Physical Review Letters, 67(6):661-663, August 1991.

[81] Ryszard Engelking. General Topology. Taylor \& Francis, 1977.

[82] Peter D. Finch. Quantum logic as an implication algebra. Bulletin of the American Mathematical Society, 11:648-654, 1960.

[83] Peter D. Finch. On the structure of quantum logic. Journal of Symbolic Logic, 34(2):275-282, 1969.

[84] Marcelo P. Fiore. Differential structure in models of multiplicative biadditive intuitionistic linear logic. In Typed Lambda Calculi and Applications, volume 4583 of Lecture Notes in Computer Science, pages 163-177. Springer, 2007.

[85] Michael P. Fourman and Robin J. Grayson. Formal spaces. In The L. E. J. Brouwer Centenary Symposium, number 110 in Studies in Logic and the Foundations of Mathematics, pages 107-122. North-Holland, 1982.

[86] Michael P. Fourman and Andre Ščedrov. The "world's simplest axiom of choice" fails. Manuscripta mathematica, 38(3):325-332, 1982.

[87] Thomas Fox. Coalgebras and cartesian categories. Communications in Algebra, 4(7):665-667, 1976.

[88] Peter Freyd. Abelian Categories: An introduction to the theory of functor. Harper and Row, 1964.

[89] Peter Freyd and Max Kelly. Categories of continuous functors I. Journal of Pure and Applied Algebra, 2, 1972.

[90] William Fulton. Young tableaux. Cambridge University Press, 1997.

[91] Israïl M. Gelfand. Normierte Ringe. Matematicheskii Sbornik, 9(51):3-24, 1941.

[92] Israil M. Gelfand and Mark A. Naimark. On the imbedding of normed rings into the ring of operators on a Hilbert space. Matematicheskii Sbornik, 12:3-20, 1943.

[93] Paul Ghez, Ricardo Lima, and John E. Roberts. $w^{*}$-categories. Pacific Journal of Mathematics, 120:79-109, 1985.

[94] Jonathan S. Golan. Semirings and their applications. Kluwer, 1999.

[95] Robert Goldblatt. Topoi. The categorical analysis of logic. North-Holland, 1984.

[96] William H. Graves and Steve A. Selesnick. An extension of the Stone representation for orthomodular lattices. Colloquium Mathematicum, 27:21-30, 1973.

[97] Phillip Griffiths and Joseph Harris. Principles of Algebraic Geometry. Wiley, 1994. 
[98] Pierre A. Grillet. Abstract Algebra. Number 242 in Graduate Texts in Mathematics. Springer, second edition, 2007.

[99] Alexandre Grothendieck. Catégories fibrées et descente (Exposé VI). In Revêtement Etales et Groupe Fondamental (SGA 1), number 224 in Lecture Notes in Mathematics, pages 145-194. Springer, 1970.

[100] Jack Gunson. On the algebraic structure of quantum mechanics. Communications in Mathematical Physics, 6:262-285, 1967.

[101] Rudolf Haag. Local quantum physics. Texts and Monographs in Physics. Springer, second edition, 1996. Fields, particles, algebras.

[102] Esfandiar Haghverdi and Phil Scott. A categorical model for the geometry of interaction. Theoretical Computer Science, 350:252-274, 2006.

[103] Phùng Hô Hài. An embedding theorem for Abelian monoidal categories. Compositio Mathematica, 132:27-48, 2002.

[104] Paul Halmos. A Hilbert space problem book. Springer, 2nd edition, 1982.

[105] Hans Halvorson and Michael Müger. Algebraic quantum field theory. In Handbook of the Philosophy of Physics, pages 731-922. North Holland, 2007.

[106] Jan Hamhalter. Traces, dispersions of states and hidden variables. Foundations of Physics Letters, 17(6):581-597, 2004.

[107] John Harding. A link between quantum logic and categorical quantum mechanics. International Journal of Theoretical Physics, 2008.

[108] Masahito Hasegawa, Martin Hofmann, and Gordan Plotkin. Finite dimensional vector spaces are complete for traced symmetric monoidal categories. In Pillars of Computer Science, number 4800 in Lecture Notes in Computer Science, pages 367-385. Springer, 2008.

[109] Ichiro Hasuo, Chris Heunen, Bart Jacobs, and Ana Sokolova. Coalgebraic components in a many-sorted microcosm. In Conference on Algebra and Coalgebra in Computer Science, Lecture Notes in Computer Science. Springer, 2009. To appear.

[110] Carsten Held. The meaning of complementarity. Studies in History and Philosophy of Science Part A, 25:871-893, 1994.

[111] Claudio Hermida. A categorical outlook on relational modalities and simulations. Information and Computation, to appear, 2009.

[112] Chris Heunen. Compactly accessible categories and quantum key distribution. Logical Methods in Computer Science, 4(4), 2008.

[113] Chris Heunen. Semimodule enrichment. In Mathematical Foundations of Programming Semantics 24, volume 218 of Electronic Notes in Theoretical Computer Science, pages 193-208. Elsevier, 2008.

[114] Chris Heunen. An embedding theorem for Hilbert categories. Theory and Applications of Categories, 22(13):321-344, 2009. 
[115] Chris Heunen and Bart Jacobs. Arrows, like monads, are monoids. In Mathematical Foundations of Programming Semantics 22, volume 158 of Electronic Notes in Theoretical Computer Science, pages 219-236. Elsevier, 2006.

[116] Chris Heunen and Bart Jacobs. Quantum logic in dagger kernel categories. In Quantum Physics and Logic, Electronic Notes in Theoretical Computer Science, 2009.

[117] Chris Heunen, Nicolaas P. Landsman, and Bas Spitters. The principle of general tovariance. In International Fall Workshop on Geometry and Physics XVI, volume 1023 of AIP Conference Proceedings, pages 93-102. American Institute of Physics, 2008.

[118] Chris Heunen, Nicolaas P. Landsman, and Bas Spitters. Bohrification of operator algebras and quantum logic. under consideration for Synthese, 2009.

[119] Chris Heunen, Nicolaas P. Landsman, and Bas Spitters. A topos for algebraic quantum theory. Communications in Mathematical Physics, 291:63-110, 2009.

[120] Karl H. Hofmann. The Duality of Compact Semigroups and C*-Bigebras, volume 129 of Lecture Notes in Mathematics. Springer, 1970.

[121] John Isbell. Some remarks concerning categories and subspaces. Canadian Journal of Mathematics, 9:563-577, 1957.

[122] Christopher J. Isham. Topos theory and consistent histories: The internal logic of the set of all consistent sets. International Journal of Theoretical Physics, 36(4):785-814, 1997.

[123] Christopher J. Isham. A topos perspective on state-vector reduction. International Journal of Theoretical Physics, 45(8):1524-1551, 2006.

[124] Bart Jacobs. Semantics of weakening and contraction. Annals of Pure and Applied Logic, 69:73-106, 1994.

[125] Bart Jacobs. Categorical Logic and Type Theory. Number 141 in Studies in Logic and the Foundations of Mathematics. North Holland, 1999.

[126] Bart Jacobs, Chris Heunen, and Ichiro Hasuo. Categorical semantics for arrows. Journal of Functional Programming, 19(3-4):403-438, 2009.

[127] Nathan Jacobson. Lectures in Abstract Algebra, volume II: Linear Algebra. Van Nostrand, Princeton, 1953.

[128] Melvin F. Janowitz. Quantifiers and orthomodular lattices. Pacific Journal of Mathematics, 13:1241-1249, 1963.

[129] Josef M. Jauch. Foundations of quantum mechanics. Addison-Wesley, 1968.

[130] Peter T. Johnstone. Stone spaces. Number 3 in Cambridge studies in advanced mathematics. Cambridge University Press, 1982.

[131] Peter T. Johnstone. Sketches of an elephant: A topos theory compendium. Oxford University Press, 2002. 
[132] André Joyal and Ross Street. An introduction to Tannaka duality and quantum groups. In Category Theory, Part II, volume 1488 of Lecture Notes in Mathematics, pages 411-492. Springer, 1991.

[133] André Joyal and Ross Street. Braided tensor categories. Advances in Mathematics, 102:20-78, 1993.

[134] André Joyal and Miles Tierney. An extension of the Galois theory of Grothendieck. Memoirs of the American Mathematical Society, 51(309), 1983.

[135] Richard V. Kadison and John R. Ringrose. Fundamentals of the theory of operator algebras. Academic Press, 1983.

[136] Gudrun Kalmbach. Orthomodular Lattices. Academic Press, 1983.

[137] Gudrun Kalmbach. Measures and Hilbert lattices. World Scientific, 1986.

[138] Irving Kaplansky. Rings of operators. W. A. Benjamin, 1968.

[139] Mikhail Kapranov and Vladimir Voevodsky. 2-categories and Zamolodchikov tetrahedra equations. In Proceedings of Symposia in Pure Mathematics. Algebraic groups and their generalizations: quantum and infinite-dimensional methods, volume 56, pages 177-259. American Mathematical Society, 1994.

[140] G. Max Kelly. Many variable functorial calculus (I). In Coherence in Categories, volume 281 of Lectures Notes in Mathematics, pages 66-105. Springer, 1970.

[141] G. Max Kelly. Basic Concepts of Enriched Category Theory. Cambridge University Press, 1982.

[142] G. Max Kelly and Miguel L. Laplaza. Coherence for compact closed categories. Journal of Pure and Applied Algebra, 19:193-213, 1980.

[143] Simon Kochen and Ernst Specker. The problem of hidden variables in quantum mechanics. Journal of Mathematics and Mechanics, 17:59-87, 1967.

[144] Anders Kock. Monads in symmetric monoidal closed categories. Archiv der Mathematik, 21(1-10), 1970.

[145] Anders Kock. Strong functors and monoidal monads. Archiv der Mathematik, 23:113-120, 1972.

[146] Anders Kock and Gonzalo E. Reyes. Doctrines in categorical logic. In Handbook of Mathematical Logic, pages 283-313. North-Holland, 1977.

[147] Joachim Kock. Frobenius algebras and 2-D Topological Quantum Field Theories. Number 59 in London Mathematical Society Student Texts. Cambridge University Press, 2003.

[148] Pavel S. Kolesnikov. Different definitions of algebraically closed skew fields. Algebra and Logic, 40(4):219-230, 2001.

[149] Mark G. Krein. A principle of duality for a bicompact group and square block algebra. Doklady Akademii Nauk SSSR, 69:725-728, 1949. 
[150] Pekka. J. Lahti. Uncertainty and complementarity in axiomatic quantum mechanics. International Journal of Theoretical Physics, 19:789-842, 1980.

[151] Joachim Lambek and Phil Scott. Introduction to higher order categorical logic. Cambridge University Press, 1986.

[152] E. Christopher Lance. Hilbert $C^{*}$-modules. Number 210 in London Mathematical Society Lecture Note Series. Cambridge University Press, 1995.

[153] Nicolaas P. Landsman. Mathematical topics between classical and quantum mechanics. Springer, 1998.

[154] F. William Lawvere. Functorial semantics of algebraic theories. Proceedings of the National Academy of Sciences, 50:869-872, 1963.

[155] F. William Lawvere. Metric spaces, generalized logic, and closed categories. Rendiconti del Seminario Matematico e Fisico di Milano, 43:135-166, 1973. Reprint in Theory and Applications of Categories 1:1-37, 2002.

[156] Daniel Lehmann. A presentation of quantum logic based on an and then connective. Journal of Logic and Computation, 18:59-76, 2008.

[157] Tom Leinster. Higher Operads, Higher Categories. Number 298 in London Mathematical Society Lecture Note Series. Cambridge University Press, 2004.

[158] Harald Lindner. Adjunctions in monoidal categories. Manuscripta Mathematica, 26:123-139, 1978.

[159] Saul Lubkin. Imbedding of Abelian categories. Transactions of the American Mathematical Society, 97:410-417, 1960.

[160] Wilhelmus A. J. Luxemburg. and Adriaan C. Zaanen. Riesz spaces. I. NorthHolland, 1971.

[161] Saunders Mac Lane. Duality for groups. Bulletin of the American Mathematical Society, 56(6):485-516, 1950.

[162] Saunders Mac Lane. An algebra of additive relations. Proceedings of the National Academy of Sciences, 47:1043-1051, 1961.

[163] Saunders Mac Lane. Categories for the Working Mathematician. Springer, 2nd edition, 1971.

[164] Saunders Mac Lane and Ieke Moerdijk. Sheaves in Geometry and Logic. Springer, 1992.

[165] George W. Mackey. Mathematical foundations of quantum mechanics. W. A. Benjamin, 1963.

[166] George W. Mackey. The theory of unitary group representations. Chicago Lectures in Mathematics. The University of Chicago Press, 1976.

[167] Leonid Makar-Limanov. Algebraically closed skew fields. Journal of Algebra, 93:117-135, 1985. 
[168] Michael Makkai and Gonzalo E. Reyes. First Order Categorical Logic. Number 611 in Lecture Notes in Mathematics. Springer, 1977.

[169] Ernest G. Manes. Algebraic Theories. Springer, 1976.

[170] Barry Mitchell. Theory of Categories. Academic Press, 1965.

[171] Roberta B. Mura and Akbar Rhemtulla. Orderable groups. Number 27 in Lecture Notes in Pure and Applied Mathematics. New York: Marcel Dekker, 1977.

[172] Sara Negri. Continuous domains as formal spaces. Mathematical Structures in Computer Science, 12(1):19-52, 2002.

[173] Michael A. Nielsen and Isaac L. Chuang. Quantum Computation and Quantum Information. Cambridge University Press, 2000.

[174] Paul H. Palmquist. Adjoint functors induced by adjoint linear transformations. Proceedings of the American Mathematical Society, 44(2):251-254, 1974.

[175] Jan Paseka. Hilbert Q-modules and nuclear ideals. In Proceedings of the Eighth Conference on Category Theory and Computer Science, volume 129 of Electronic Notes in Theoretical Computer Science, pages 1-19, 1999.

[176] Zoran Petric. Coherence in substructural categories. Studia Logica, 70(2):271296, 2002.

[177] Constantin Piron. Foundations of quantum physics. Number 19 in Mathematical Physics Monographs. W.A. Benjamin, 1976.

[178] Lew Pontrjagin. Über stetige algebraischer körper. Annals of Mathematics, 33:163174, 1932.

[179] Dieter Puppe. Korrespondenzen in abelschen Kategorien. Mathematische Annalen, 148:1-30, 1962.

[180] Miklós Rédei. Quantum Logic in Algebraic Approach. Kluwer, 1998.

[181] Michael Reed and Barry Simon. Methods of Modern Mathematical Physics, Vol I: Functional Analysis. Academic Press, 1972.

[182] Charles E. Rickart. General theory of Banach algebras. D. van Nostrand, 1960.

[183] Leopoldo Román. A characterization of quantic quantifiers in orthomodular lattices. Theory and Applications of Categories, 16(10):206-217, 2006.

[184] Leopoldo Román and Beatriz Rumbos. A characterization of nuclei in orthomodular and quantic lattices. Journal of Pure and Applied Algebra, 73:155-163, 1991.

[185] Leopoldo Román and Rita E. Zuazua. On quantic conuclei in orthomodular lattices. Theory and Applications of Categories, 2(6):62-68, 1996.

[186] Mikael Rørdam. Structure and classification of $\mathrm{C}^{*}$-algebras. In Proceedings of the International Congress of Mathematicians, volume 2, pages 1581-1598. EMS Publishing House, 2006. 
[187] Robert Rosebrugh and Richard J. Wood. Distributive laws and factorization. Journal of Pure and Applied Algebra, 175:327-353, 2002.

[188] Neantro Saavedra Rivano. Catégories Tannakiennes. Number 265 in Lecture Notes in Mathematics. Springer, 1972.

[189] Mehrnoosh Sadrzadeh. High level quantum structures in linguistics and multiagent systems. In AAAI Spring symposium on quantum interactions. Stanford University, 2007.

[190] Shôichirô Sakai. $C^{*}$-algebras and $W^{*}$-algebras. Springer, 1971.

[191] Giovanni Sambin. Intuitionistic formal spaces - a first communication. In D. Skordev, editor, Mathematical logic and its Applications, pages 187-204. Plenum, 1987.

[192] Giovanni Sambin. Some points in formal topology. Theoretical Computer Science, 305:347-408, 2003.

[193] Erhard Scheibe. The logical analysis of quantum mechanics. Pergamon, 1973.

[194] Dana Scott. Lattice theory, data types and semantics. In NYU Symposium on formal semantics, pages 65-106. Prentice-Hall, 1972.

[195] Robert A. G. Seely. Linear logic, *-autonomous categories and cofree coalgebras. In Categories in Computer Science and Logic, volume 92, pages 371-382. American Mathematical Society, 1989.

[196] Irving E. Segal. Postulates for general quantum mechanics. Annals of Mathematics, 48:930-948, 1947.

[197] Peter Selinger. Dagger compact closed categories and completely positive maps. In Quantum Programming Languages, volume 170 of Electronic Notes in Theoretical Computer Science, pages 139-163. Elsevier, 2007.

[198] Peter Selinger. Finite dimensional Hilbert spaces are complete for dagger compact closed categories. In Quantum Physics and Logic, Electronic Notes in Theoretical Computer Science, 2008.

[199] Peter Selinger. Idempotents in dagger categories. In Quantum Programming Languages, volume 210 of Electronic Notes in Theoretical Computer Science, pages 107122. Elsevier, 2008.

[200] Peter Selinger. A survey of graphical languages for monoidal categories. In New Structures for Physics, Lecture Notes in Physics. Springer, 2009.

[201] Maria P. Solèr. Characterization of Hilbert spaces by orthomodular spaces. Communications in Algebra, 23:219-243, 1995.

[202] Bas Spitters. Constructive results on operator algebras. Journal of Universal Computer Science, 11(12):2096-2113, 2005.

[203] Serban Stratila and Laszlo Zsido. Operator Algebras. Theta Foundation, 2009. 
[204] Ross Street. Quantum Groups: a path to current algebra. Number 19 in Australian Mathematical Society Lecture Series. Cambridge University Press, 2007.

[205] Isar Stubbe. The canonical topology on a meet-semilattice. International Journal of Theoretical Physics, 44:2283-2293, 2005.

[206] Masamichi Takesaki. Theory of Operator Algebra I. Encyclopaedia of Mathematical Sciences. Springer, 1979.

[207] Tadao Tannaka. Über den Dualitätssatz der nichtkommutatieven topologischen Gruppen. Tôhoku Mathematical Journal, 45:1-12, 1939.

[208] Paul Taylor. Practical Foundations of Mathematics. Number 59 in Cambridge Studies in Advanced Mathematics. Cambridge University Press, 1999.

[209] Veeravalli S. Varadarajan. Geometry of Quantum Theory. D. van Nostrand, 1968.

[210] Jamie Vicary. Categorical formulation of quantum algebras. arXiv:0805.0432 2008.

[211] Jamie Vicary. Categorical properties of the complex numbers. arXiv:0807.2927 2008.

[212] Steven Vickers. Locales and toposes as spaces. In Handbook of Spatial Logics, pages 429-496. Springer, 2007.

[213] John von Neumann. Mathematische Grundlagen der Quantenmechanik. Springer, 1932.

[214] Joachim Weidmann. Lineare Operatoren in Hilberträumen I. Grundlagen. B. G. Teubner, 2000.

[215] Edwin Weiss and Neal Zierler. Locally compact division rings. Pacific Journal of Mathematics, 8(2):369-371, 1958.

[216] William K. Wootters and Wojciech H. Zurek. A single quantum cannot be cloned. Nature, 299:802-803, 1982.

[217] Adriaan C. Zaanen. Riesz spaces. II. North-Holland, 1983.

[218] Elias Zafiris. Boolean coverings of quantum observable structure: a setting for an abstract differential geometric mechanism. Journal of Geometry and Physics, 50(1-4):9-114, 2004. 



\section{Index of categories}

\begin{tabular}{|c|c|c|}
\hline Notation & Description & Page(s) \\
\hline Act & Actions of a monoid & 17,32 \\
\hline $\operatorname{Alg}(T)$ & Eilenberg-Moore $T$-algebras & \\
\hline BP & Categories with finite biproducts & \\
\hline$\widehat{B}$ & Boolean algebra $B$ as dagger kernel category & 130 \\
\hline$[\mathbf{C}, \mathbf{D}]$ & Functors $\mathbf{C} \rightarrow \mathbf{D}$ and natural transformations & 18,53 \\
\hline $\mathbf{C}_{\leftrightarrows}$ & Cofree dagger category on $\mathbf{C}$ & $25, \overline{54}$ \\
\hline Cat & Categories and functors & 13 \\
\hline CHey & Complete Heyting algebras & $1 \overline{42}$ \\
\hline CStar & $C^{*}$-algebras and *-morphisms & 151 \\
\hline DagCat & Dagger categories and dagger functors & \\
\hline DagKerCat & Dagger kernel categories & \\
\hline $\mathbf{D}_{\text {kck }}$ & Kck-construction on D & $1 \overline{31}$ \\
\hline finPInj & Finite sets and partial injections & 53,61 \\
\hline Frm & Frames & \\
\hline Hilb & Hilbert spaces and continuous linear maps & \\
\hline HMod & Hilbert modules and adjointable morphisms & \\
\hline InvAdj & Involutive categories and contravariant adjunctions & \\
\hline InvGal & Orthoposets and antitone Galois connections & \\
\hline IPOSet & Indexed partially ordered sets & $\overline{126}$ \\
\hline KRegLoc & Compact regular locales & \\
\hline Loc & Locales & \\
\hline $\operatorname{Loc}(\mathbf{T})$ & Locales in $\mathbf{T}$ & \\
\hline Mod & Modules and linear transformations & 36 \\
\hline Mon & Monoids & 15 \\
\hline OMLatGal & Orthomodular lattices and antitone Galois connections & \\
\hline PHilb & Hilbert spaces and continuous linear maps up to phase & 13 \\
\hline PInj & Sets and partial injections & \\
\hline POSet & Partially ordered sets & 126 \\
\hline
\end{tabular}


preHilb Pre-Hilbert spaces and adjointable functions

Rel

Sets and relations

Rg

Rigs

Set

Sets and functions

Topos Toposes and geometric morphisms

Vect

Vector spaces and linear transformations

V-Cat V-enriched categories and functors

$\operatorname{Zigzag}(\mathbf{C}) \quad$ Free dagger category on $\mathbf{C}$ 


\section{Index of notation}

$A_{\text {sa }}$, self-adjoints, 152

$\operatorname{Alx}(P)$, Alexandrov topology, 143

$B[m]$, order generated by $B, m, 105$

$C(X, Y)$, locale morphisms, 142

$C^{*}(a), C^{*}$-algebra generated by $a, 173$

$I$, monoidal unit, 14

$\operatorname{Im}(f)$, image of $f$, 75

KSub, kernels, 100, 122, 126

$L_{A}$, lattice generating spectrum, 153

$P(m)$, projection, 102

$\operatorname{Proj}(X)$, projections, 102

$R^{+}$, positive cone, 80,153

$\operatorname{Sub}(X)$, subobjects, 100

$U(1)$, circle group, 13,158

$X^{*}$, dual object, 42

$e_{f}$, coimage of $f, 75$

$f^{*}$, continuous map, 144

$f^{*}$, dual morphism, 45

$f^{*}$, left adjoint, 50,113

$f_{*}$, dual adjoint morphism, 59

$f_{*}$, right adjoint, 50, 113

$i_{f}$, image of $f$, 75

$\mathcal{A}\left(\_\right)$, Boolean amalgamation, 169

$\mathcal{B}\left(\_\right)$, Heyting amalgamation, 169

$\mathcal{C}\left(\_\right)$, contexts, 156, 157, 166

$\mathcal{F}(L, \triangleleft)$, free frame, 144

$\mathcal{I}\left(\_\right)$, probability integrals, 174

$\mathcal{O}(X)$, frame, 142

$\mathcal{T}\left({ }_{-}\right)$, Bohrification topos, 156 , 157 ,
166

$\mathfrak{T}$, theory, 148

$\mathcal{V}\left(\_\right)$, probability valuations, 175

$\Delta$, diagonal, 23

$\nabla$, codiagonal, 23

$\Omega$, subobject classifier, 133

$\Sigma$, Gelfand spectrum, 152

$\Sigma_{A}$, Bohrified state space, 162

$\alpha$, coherence isomorphism, 14

$\delta$, classical structure, 68

$\varepsilon$, counit of compactness, 42

$\eta$, unit of compactness, 42

$\gamma$, symmetry isomorphism, 14

$\lambda$, coherence isomorphism, 14

$\nu$, classical structure, 68

$\rho$, coherence isomorphism, 14

$\tau$, distributivity isomorphism, 30

$\mathbb{B}$, Boolean rig, 30, 34

$\mathbb{C}$, complex numbers, 13, 20, 60, 151

$\mathbb{C}_{\mathbb{Q}}$, Gaussian rationals, 150

$\mathbb{H}$, quaternions, 13,89

$\mathbb{I R}$, interval domain, 175

$\mathbb{R}$, real numbers, $13,150,174$

$\left({ }^{\prime}\right)^{*}$, involution, 150

-, scalar multiplication, 16

†, dagger, 47

$\ddagger$, involution, 12,80

$[f, g]$, cotuple, 23

$\Rightarrow$, implication, 118 


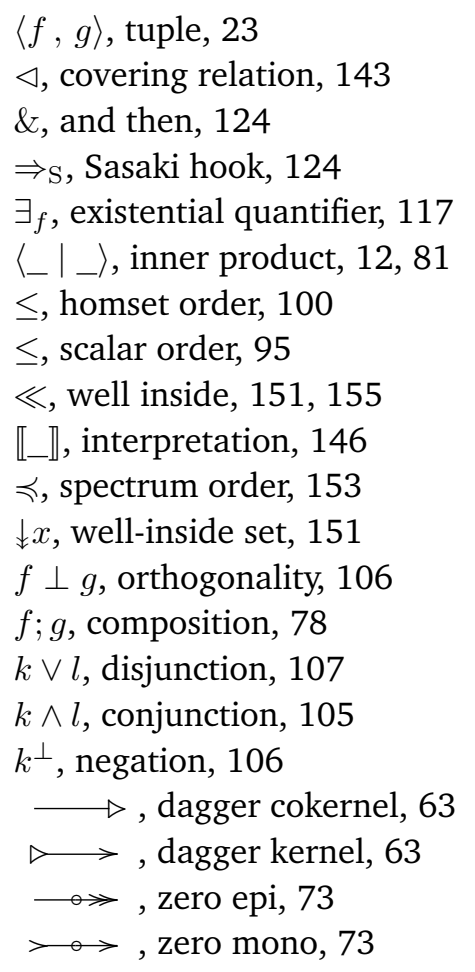




\section{Index of subjects}

action, 17

adjoint morphism, 12, 47

Alexandrov topology, 143

and then, 124

atom, 104

atomic, 104

atomistic, 104

bimorphism, 20

biproduct, 24, 26

bounded, 28

Bohrification, 159, 166

Bohrified state space, 162

Boolean algebra, 128

Boolean category, 127

Boolean rig, 30, 34

bounded morphism, 95

Bruns-Lakser completion, 171

C*-algebra, 151

Rickart, 163

C*-category, 97

Cauchy completion, 52,94

characters, 152

choice-of-duals functor, 45

classical structure, 68

closure operation, 103, 144

coaffine, 61

coimage, 77

cokernel, 35 compact closed category, 42

compact object, 42

compact structure, 42

coname, 44

continuous probability valuation, 175

cosieve, 133

covering relation, 143

dagger, 47

dagger biproduct, 61

dagger category, 47

cofree, 25,54

free, 55

dagger coequaliser, 63

dagger cokernel, 63

dagger compact closed category, 59

dagger compact object, 59

dagger epi, 57

dagger equaliser, 62

dagger equaliser category, 63

dagger functor, 47

dagger iso, 57

dagger kernel, 62

dagger kernel category, 63

Boolean, 127

dagger monoidal category, 59

dagger regular category, 77

Daseinisation, 177, 179

Dedekind real numbers, 149, 174, 176

diagonal fill-in, 75 
dimension, 44

directed colimit, 109

distributive lattice, 119, 153

normal, 155

division rig, 87

dual object, 42

enriched monoidal category, 16

extension of scalars, 91

f-algebra, 154

factorisation system, 74

dagger, 75

finitely projective, 44

frame, 142

Frobenius condition, 68

Galois connection, 50

Gelfand duality, 151

Gelfand spectrum, 152, 155, 162, 167

generator, 36, 104

geometric formula, 148, 153

geometric morphism, 145

Grothendieck completion, 32, 125

Heyting algebra, 118

Hilbert category, 95

Hilbert module, 81

strict, 81

Hilbert space, 13

Hilbert-Schmidt function, 61

ideal, 155

distributive, 171

regular, 155

image, 76

inner product, 12,81

integral, 173

interval domain, 175

kernel, 35

kernel subobject, 100
Kock-Day tensor product, 19

Kripke topos, 145

Kripke-Joyal semantics, 146

locale, 142

compact, 151

regular, 151

Lubkin, 39

measurement, 69

Mitchell-Bénabou language, 146

module, 11, 34

finitely projective, $12,36,44$

over a rig, 34

monad, 18

commutative, 19

strong, 18

monoid, 14

monoidal category, 14

name, 44

open, 143

orthocomplemented lattice, 107

orthogonal, 106, 127

orthogonal kernel subobject, 106

orthomodular lattice, 111

orthoposet, 50

partial Boolean algebra, 168

partial isometry, 79

point, 142

positive, 80, 153

pre-Hilbert category, 90

pre-Hilbert space, 12

probability measure, 176

probability valuation, 176

projection, 57, 102

projective, 36,44

pseudocomplement, 166

quantale, 81,125 
quantum key distribution, 66

quasi-state, 173

regular category, 39

restriction of scalars, 92

Riesz space, 153

rig, 30

involutive, 80

multiplicatively cancellative, 81

Sasaki hook, 124, 170

scalar, 17

scalar multiplication, 17

Scott topology, 175

self-adjoint morphism, 57, 152

seminorm, 150

sheaf, 145

Sierpinski space, 143

simple, 86, 104, 161

state, 172

subfunctor, 133

subobject, 86,100

subobject classifier, 132

suitable, 20

support, 20, 164

topos, 145

truth value, 146

upper set, 133

zero epi, 73

zero mono, 73

zero object, 24

zerosumfree, 83 



\section{Samenvatting}

Kwantummechnica is de beste beschikbare beschrijving van de natuur op hele kleine schaal. Computers worden steeds kleiner, en kunnen niet veel langer kwantumwetten negeren. Gebruik van kwantummechanische principes levert dan ook vele voordelen, zoals computers die essentieel sneller zijn dan klassieke, en communicatieprotocollen waarbij afluisteren in principe onmogelijk is.

Maar de drie belangrijkste kwantummechanische principes, superpositie, entanglement, en niet-commutativiteit, druisen regelrecht in tegen onze intuïtie, opgedaan op de grotere schaal van het dagelijkse leven. Superpositie, bijvoorbeeld, houdt in dat een kwantumbit níet ofwel 0 ofwel 1 hoeft te zijn, zoals een klassiek bit, ook al is het resultaat van een meting wel altijd ofwel 0 ofwel 1 . Entanglement is de onbegrijpelijke eigenschap dat kwantumbits zo verstrengeld kunnen raken, dat zodra de ene gemeten wordt, de uitslag van een meting van de ander daarmee direct vastligt, zelfs als ze heel ver uiteen liggen, en zelfs zonder gemeenschappelijke oorzaak van de uitslagen. Niet-commutativiteit, tenslotte, zorgt ervoor dat we niet alle eigenschappen van een kwantumdeeltje tegelijktertijd kunnen meten: eerst snelheid en dan positie meten geeft een andere uitkomst dan andersom.

Omdat onze intuitie ons in dezen in de steek laat, zijn rigoureuze wiskundige bewijzen noodzakelijk: niemand zal een kwantumcomputer serieus gebruiken als de programmeur niet in kan staan voor de correctheid van het programma, en de aantrekkelijkheid van kwantumcommunicatieprotocollen schuilt juist in de garantie dat afluisteren onmogelijk is. Met andere woorden, er is een logica nodig voor kwantumtheorie, en daar gaat dit proefschrift over.

Om de aard van zo'n logica te begrijpen, bestuderen we niet één enkele geïsoleerde toestandsruimte, maar alle toestandsruimtes tegelijk, en de relaties tussen verschillende toestandsruimtes. Dit heet een categorie. Zo kunnen we het gedrag van kwantummechanische systemen kwalitatief bestuderen, zonder te hoeven gokken hoe ze in elkaar zitten, want dat weten we immers niet.

De eerste helft van dit proefschrift bestudeert categorieën die voldoen aan eisen die bovengenoemde kwantummechanische principes modelleren, zoge- 
heten categorische kwantum modellen. Hoofdstuk 2 modelleert superpositie en de eis dat twee aparte systemen samen als één groter systeem gezien kunnen worden. Hoofdstuk 3 eist daar bovenop dat iedere relatie tussen toestandsruimten omkeerbaar is, wat nodig is om kwantumcomputers aan de wet van behoud van energie te laten voldoen. Met alleen deze axioma's kunnen we dan al bewijzen dat elk zo'n categorie in te bedden is in de categorie van Hilbertruimten, die het traditionele natuurkundige model vormt voor kwantummechanica. Zo vinden we bijvoorbeeld dat—ook al is daar niets over geëist—zo'n categorie altijd scalairen heeft, die zich gedragen als complexe getallen.

De tweede helft richt zich dan op de logica in deze categorische modellen, zogeheten categorische logica. Hoofdstuk4 laat zien dat, wanneer je logica direct in zulke categorische modellen probeert te interpreteren, deze dezelfde vreemde eigenschappen heeft als wat traditioneel kwantumlogica genoemd wordt. Bijvoorbeeld de logische connectieven 'en' en 'of'; in het dagelijks leven geldt

$$
x \text { en }(y \text { of } z)=(x \text { en } y) \text { of }(x \text { en } z)
$$

voor willekeurige uitspraken $x, y$ en $z$. Maar bij een kwantumontbijt, waarbij je de keuze krijgt tussen een ei $(x)$ met ofwel spek $(y)$ ofwel ham $(z)$, kan het gebeuren dat je noch ei met spek, noch ei met ham voorgeschoteld wordt. Ook uitspraken als 'als ..., dan ...' zijn alleen zinnig in speciale gevallen. Er wordt echter ook duidelijk hoe existentiële kwantificatoren, zoals 'er is een $x$ zodat ...' gedefinieerd en gebruikt kunnen worden. We bewijzen ook dat universele kwantificatie, zoals 'voor alle $x$ geldt ...', onmogelijk is. Dit bleef onduidelijk in de traditionele kwantumlogica, maar wordt verhelderd door ons gebruik van categorieën. Het blijkt dat deze kwantificaties een dynamisch, temporeel karakter hebben, dat veroorzaakt wordt door niet-commutativiteit.

Tenslotte omzeilen we in hoofdstuk 5 het probleem van niet-commutativiteit door categorische logica op een geraffineerdere manier te gebruiken. Hiervoor zijn twee ingrediënten van belang. Ten eerste is een $C^{*}$-algebra een eenvoudig voorbeeld van onze kwantummodellen: commutatieve $\mathrm{C}^{*}$-algebras modelleren klassieke systemen, en niet-commutatieve $\mathrm{C}^{*}$-algebras modelleren kwantumsystemen. Ten tweede is een topos een categorie waarin 'normale' (intuitionistische) logica geïnterpreteerd kan worden. Gegeven een niet-commutatieve $\mathrm{C}^{*}$ algebra, construeren we een topos, waarin de $\mathrm{C}^{*}$-algebra commutatief wordt. Wiskundig gezien relateren we zo de twee generalisaties van het begrip van (topologische) ruimte. Logisch gezien is het grote voordeel dat we, met de logica van de topos, de $\mathrm{C}^{*}$-algebra dus kunnen bestuderen alsof ze een klassiek systeem beschrijft. 


\section{Curriculum vitae}

Chris Heunen was born on the 21st of March 1982 in Nijmegen, the Netherlands. He grew up in Venlo, returning to Nijmegen in 1999 to begin the studies of computer science and mathematics at the Radboud University. After spending a semester at the University of British Columbia in Vancouver, Canada, in 2004, he obtained M.Sc. degrees in computer science and mathematics in 2005, both cum laude. In August 2005, Chris became a Ph.D. student at the Radboud University Nijmegen, jointly supervised by professors Bart Jacobs and Klaas Landsman. The topic of this Ph.D. thesis is a combination of their fields of expertise: categorical logic and mathematical physics, respectively. As of August 2009, the author is a postdoctoral researcher at the University of Oxford, financially supported by an NWO Rubicon grant. 